University of Louisville

ThinkIR: The University of Louisville's Institutional Repository

Electronic Theses and Dissertations

$12-2019$

\title{
Electrochemical determination of surface area-to-volume ratio for metal nanoparticle analysis.
}

Jay Narayan Sharma

University of Louisville

Follow this and additional works at: https://ir.library.louisville.edu/etd

Part of the Chemistry Commons

\section{Recommended Citation}

Sharma, Jay Narayan, "Electrochemical determination of surface area-to-volume ratio for metal nanoparticle analysis." (2019). Electronic Theses and Dissertations. Paper 3304.

https://doi.org/10.18297/etd/3304

This Doctoral Dissertation is brought to you for free and open access by ThinkIR: The University of Louisville's Institutional Repository. It has been accepted for inclusion in Electronic Theses and Dissertations by an authorized administrator of ThinkIR: The University of Louisville's Institutional Repository. This title appears here courtesy of the author, who has retained all other copyrights. For more information, please contact thinkir@louisville.edu. 


\title{
ELECTROCHEMICAL DETERMINATION OF SURFACE AREA- TO-VOLUME RATIO FOR METAL NANOPARTICLE ANALYSIS
}

\author{
Jay Narayan Sharma \\ A Dissertation \\ Submitted to the Faculty of the \\ College of Arts and Sciences of the University of Louisville \\ in Partial Fulfillment of the Requirements for the Degree of
}

Doctor of Philosophy in Chemistry

\author{
Department of Chemistry \\ University of Louisville \\ Louisville, Kentucky
}

December 2019 
Copyright 2019 by Jay Narayan Sharma

All rights reserved 



\title{
ELECTROCHEMICAL DETERMINATION OF SURFACE AREA-TO-VOLUME RATIO FOR METAL NANOPARTICLE ANALYSIS
}

\author{
By \\ Jay Narayan Sharma \\ A Dissertation Approved on \\ July $26^{\text {th }}, 2019$ \\ by the following Dissertation Committee:
}

Dissertation Director - Dr. Francis P. Zamborini

Dr. M. Cecilia Yappert

Dr. Farshid Ramezanipour

Dr. Gamini Sumanasekera 
"Failure will never overtake me if my determination to succeed is strong enough." - Og Mandino

This dissertation is dedicated to my loving parents especially to my belated and extremely supportive father - Dr. Narayan Sharma. 


\section{ACKNOWLEDGEMENTS}

I would like to express my gratitude to my advisor, Dr. Francis P. Zamborini for his guidance, support and ever helping nature without which this work would not have been possible. His words of motivation during the challenging phases of this work has helped me a lot in coming over those times.

I am extremely thankful to Dr. M. Cecilia Yappert, Dr. Farshid Ramezanipour and Dr. Gamini Sumanasekera for agreeing to be a part of my dissertation committee members. I am indebted to them for their valuable input and feedback on my research projects.

I am thankful to all my former lab mates and current lab mates for their help at various stages of my graduate studies. I am especially thankful to my colleagues Dr. Dhruba Pattadar and Badri Mainali who helped me in the initial few months of my project. I would also like to acknowledge Huson Nanotechnology Core Facility at the University of Louisville for allowing access to the Scanning Electron Microscope.

Finally, I am extremely thankful to my family and friends. I am eternally grateful to my parents Dr. Narayan Sharma and Vimala Sharma for allowing me to travel to the United States to pursue my dream of doctoral studies and more importantly for their constant support and patience during this journey. I would also like to express special thanks to all my three sisters, my brother-in-laws, my 
brother, and my sister-in-law, who all encouraged me in following my goals. A special thanks to my wife Neha for being patient and allowing me to work for long hours in the lab. 


\begin{abstract}
ELECTROCHEMICAL DETERMINATION OF SA-TO-VOLUME RATIO FOR METAL NANOPARTICLE ANALYSIS
\end{abstract}

Jay Narayan Sharma

July $26^{\text {th }}, 2019$

This dissertation describes an electrochemical approach for measuring the surface area-to-volume ratio (SA/V) of electrode-attached metal nanoparticles (NPs), which was used to analyze their size, aggregation state, and porosity. This dissertation further describes the effect of the metal NP electrode assembly method on the SA/V, which is related to metal NP catalytic activity and stability.

Cyclic voltammetry (CV) in acid electrolyte followed by anodic stripping voltammetry (ASV) in $\mathrm{KBr}$ electrolyte allows the electrochemical measurement of the SA/V of electrode-attached Au nanospheres (NSs). InCV, the forward scan produces a thin surface $\mathrm{Au}_{2} \mathrm{O}_{3}$ layer on the Au NSs. Measuring the Coulombs of charge passed during the reduction of the $\mathrm{Au}_{2} \mathrm{O}_{3}$ on the reverse scan allows a measurement of the total surface area (SA) of all NSs on the electrode. Subsequent measurement of the Coulombs of charge passed during oxidative dissolution of all of the Au NSs in $\mathrm{KBr}$ electrolyte provides a measurement of the total $V$ of the Au NSs. Since the radius of a sphere is equal to $3 /(\mathrm{SA} / \mathrm{V})$, the SA/V provides a direct measure of the Au NS size, which matches very closely to the 
size of several Au NSs ranging from $4 \mathrm{~nm}$ to $70 \mathrm{~nm}$ in diameter based on scanning electron microscopy (SEM) size measurements..

Electrochemical SA/V measurements provide information about the aggregation state of electrode-attached Au NSs. The SA/V decreases as $4 \mathrm{~nm}$ and $15 \mathrm{~nm}$ diameter Au NSs go from isolated NSs to aggregated structures, caused by the neutralization of the citrate stabilizer at low $\mathrm{pH}$. The decreased SA/V for aggregated Au NSs accounts for the positive shift in the peak oxidation potential $\left(E_{p}\right)$ of aggregated NSs compared to isolated ones.

The SA/V of distorted, flattened, and porous Au NSs formed by dealloying Ag from AuAg alloy NSs increases with an increasing percentage of Ag in the initial alloy NSs. Greater removal of Ag leads to greater porosity and a higher SA/V. The increased SA/V for porous NSs resulted in a negative shift in $E_{p}$ relative to similar diameter non-porous Au NSs.

The SA/V of similar sized NSs assembled onto indium-tin-oxide-coated glass electrodes (glass/ITO) by different assembly methods, including electrostatic attachment to an amine-functionalized silane linker, electrophoretic deposition (EPD), direct drop-cast deposition, and drop-cast deposition after mixing with carbon black, can be dramatically different. Au NSs with higher SA/V generally exhibit higher electrocatalytic activity, as demonstrated for the oxygen reduction reaction (ORR), but also oxidize at lower potentials (lower $\left.E_{p}\right)$. The SA/V follow the order of electrostatic attachment $>$ EPD $>$ drop-cast with carbon black > drop-cast. Since different assembly methods lead to different SA/V for the same size Au NSs, 
the $S A / V$ is a better predictor of $E_{p}$, or the oxidation properties of the NSs, compared to the size (or curvature) of the Au NSs. This is generally true for all of the different variables studied in this dissertation, including NSs of different size, aggregation state, porosity, and assembled by different methods. The SA/V provides important information about both activity and stability of metal NPs. 
TABLE OF CONTENTS

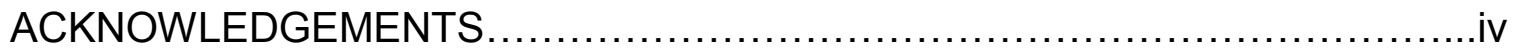

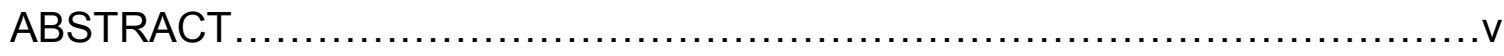

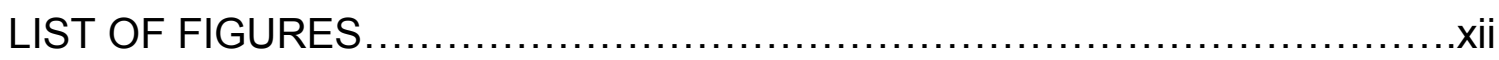

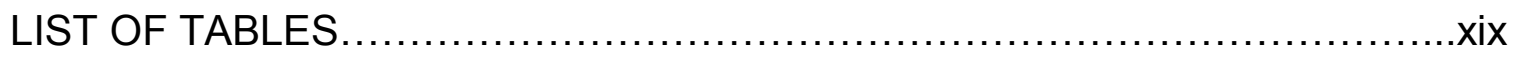

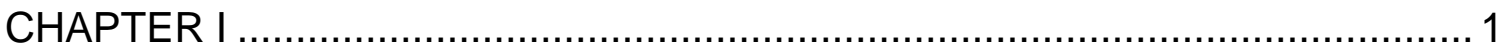

INTRODUCTION

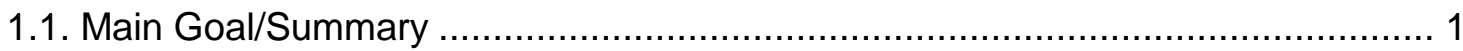

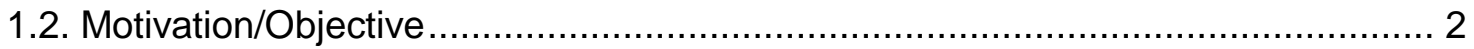

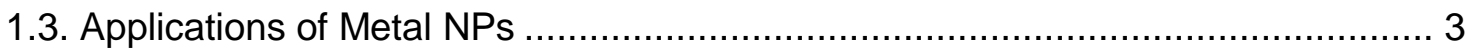

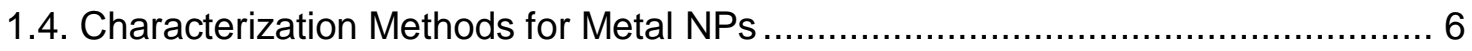

1.4.1. Electron and scanning probe microscopy methods.................................... 7

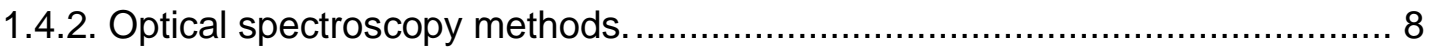

1.4.3. Electrochemical methods for NP analysis............................................ 9

1.5. Surface Area Measurements of NPs ........................................................... 13

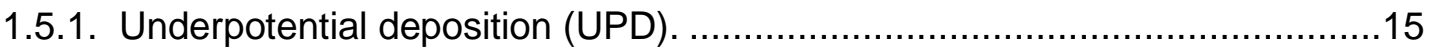

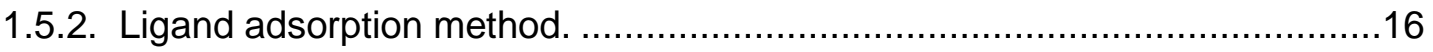

1.5.3. The Brunauer-Emmett-Teller (BET) adsorption method. ...........................17

1.6. Size-Dependent Oxidation and Stability of Metal NPs .......................................19

1.7. Importance of SA-to-Volume Ratio (SA/V) in the Analysis of Metal NPs.............22

1.7.1. Electrochemical SA/V for Size Analysis of Metal NPs...............................23

1.7.2. Electrochemical $S A / V$ for Aggregation Analysis of Metal NPs.....................25

1.7.3. Electrochemical SA/V Determination of Metal NPs Assembled by Different Assembly Methods on Glass/ITO Electrode. .....................................................28

1.7.4. Electrochemical SA/V for Analysis of Porous Metal NPs.............................30

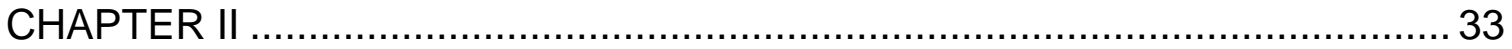

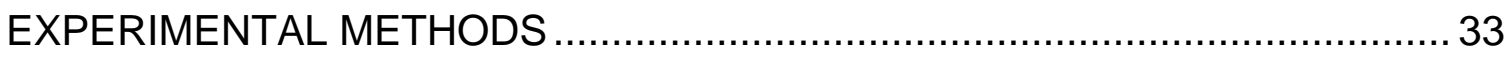

2.1. Indium-Tin-Oxide-Coated Glass Electrodes (glass/ITO)..................................34 


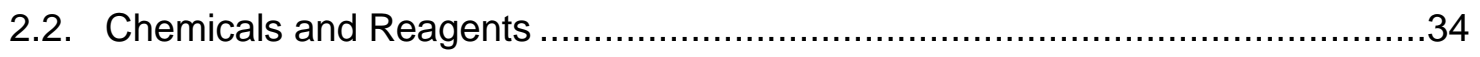

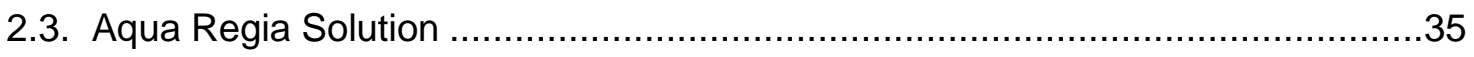

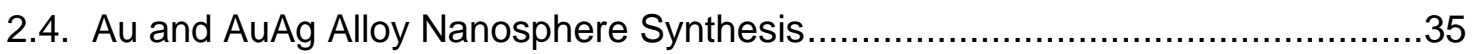

2.4.1. Synthesis of $1.6 \mathrm{~nm}$ Average Diameter THPC-Stabilized Au Nanospheres

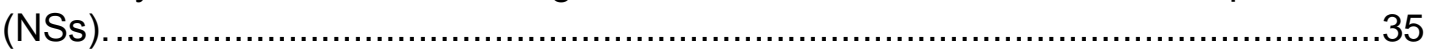

2.4.2. Synthesis of $4 \mathrm{~nm}$ Average Diameter Citrate-Stabilized Au NSs. ...................36

2.4.3. Synthesis of $15 \mathrm{~nm}$ Average Diameter Citrate-Stabilized Au NSs.................37

2.4.4. Synthesis of 32,50 and $70 \mathrm{~nm}$ Average Diameter Citrate-Stabilized Au NSs.

An Average Diameter Citrate-Stabilzed AuNS.

2.5 Electrode Functionalization and Nanoparticle Attachment to Glass/ITO .

2.5.1. Functionalization of glass/ITO electrodes with 3-aminopropyltriethoxysilane (APTES)

2.5.2. Attachment of Au NSs to glass/ITO functionalized with APTES linker...........40

2.5.3. Attachment of Au NSs to glass/ITO by drop cast deposition. ........................40

2.5.4. Electrophoretic deposition (EPD) of Au NPs directly on glass/ITO..............41

2.6. Characterization and Instrumentation ....................................................... 43

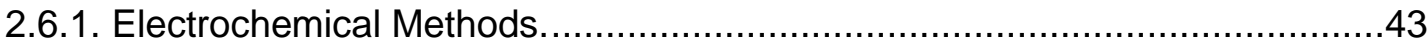

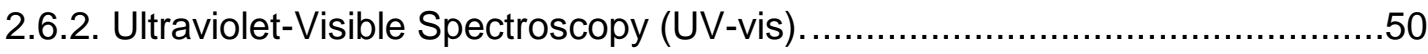

2.6.3. Scanning Electron Microscopy (SEM) .................................................... 51

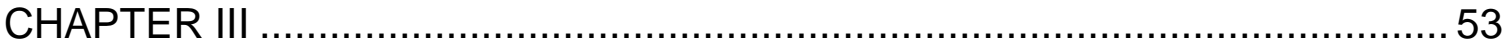

Size Determination of Metal NPs Based on Electrochemically Measured Surface

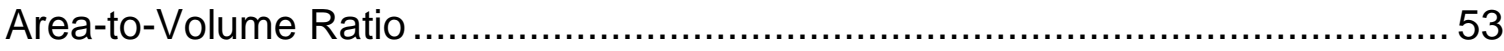

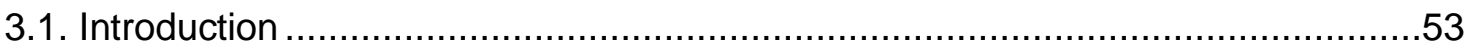

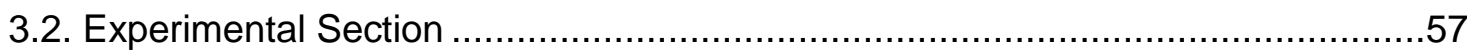

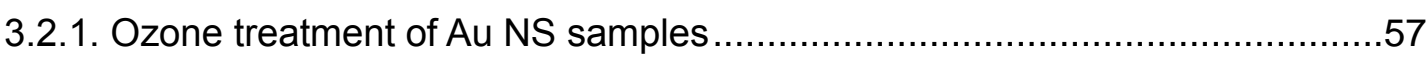

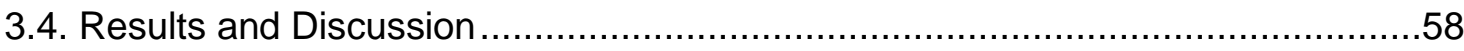

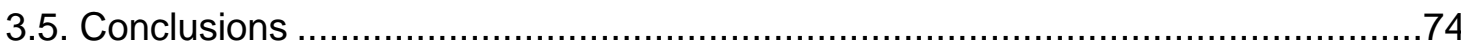

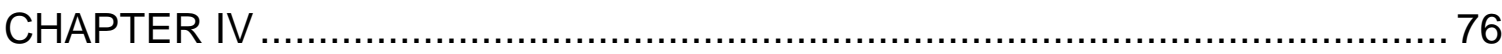

Electrochemical Surface Area-to-Volume Ratio (SA/V) of different aggregated states of Au NSs ...................................................................................... 76

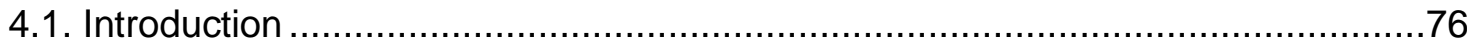

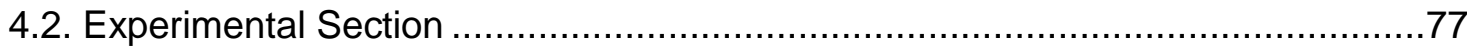

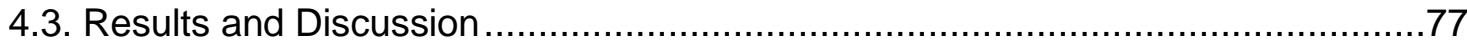

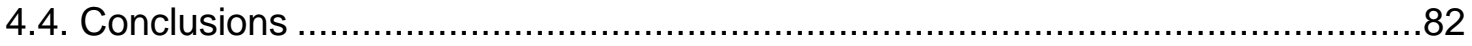


The Impact of Assembly Method on the Surface Area-to-Volume Ratio and Electrochemical Oxidation Potential of Metal NPs

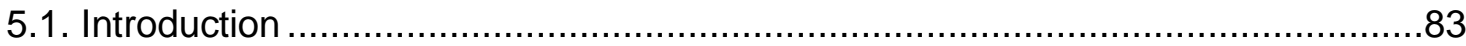

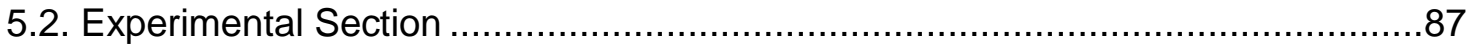

5.2.1 Synthesis of $1.6 \mathrm{~nm}$ Average Diameter THPC Stabilized Au Nanospheres (NSs).

5.2.2. Drop-cast deposition of $1.6 \mathrm{~nm}, 4 \mathrm{~nm}$ and $15 \mathrm{~nm}$ Au NPs mixed with different concentrations of carbon black (CB) directly on Glass/ITO .................................87

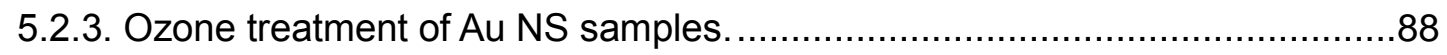

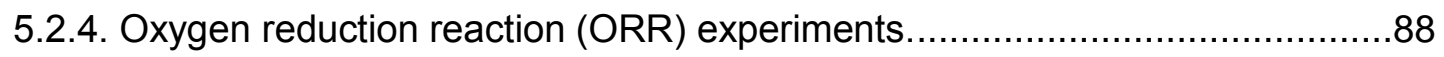

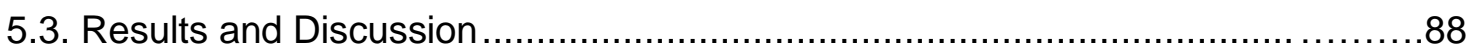

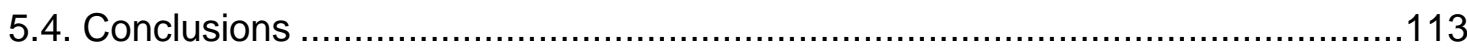

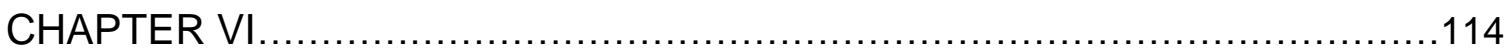

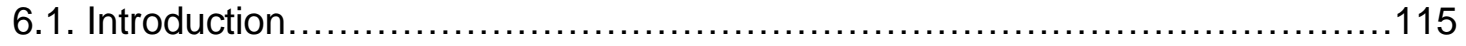

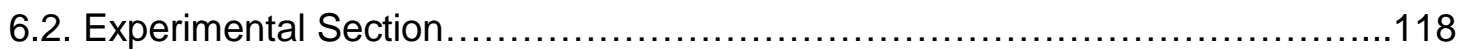

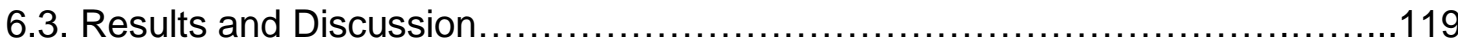

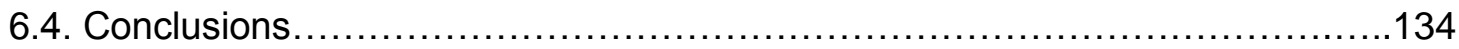

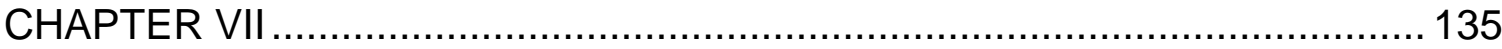

Summary, Conclusions and Future Directions............................................. 135

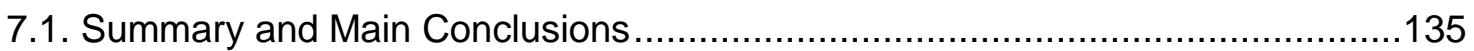

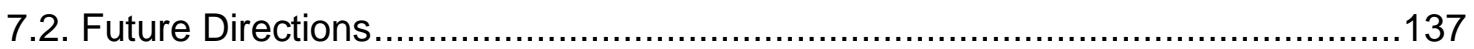

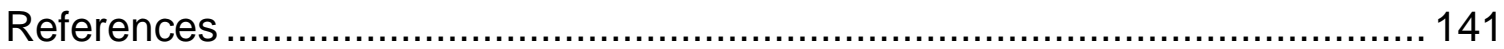

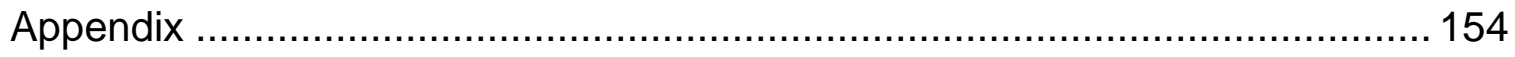

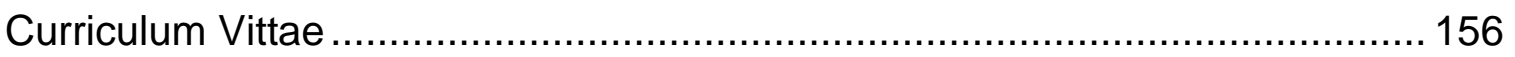




\section{LIST OF FIGURES}

Figure 1. 1 Schematic representation showing the increase in SA (SA), edge lengths (el), corner (c), and terrace (ta) sites with a decrease in cube size (edge length, L) of NPs by breaking up 1 cube into 8 cubes and then into 64 cubes. .. 13

Figure 1. 2. Schematic representation of the principle involved in determination of SA (SA) by underpotential deposition (UPD). The peak at $0.190 \mathrm{~V}$ and $0.450 \mathrm{~V}$ in the scan direction from $0.8 \mathrm{~V}$ to $-0.2 \mathrm{~V}$ in cyclic voltammetry indicates the formation of $\mathrm{Pb}$ monolayer on the nanoporous $\mathrm{Au}$ electrode, while the same peaks in the scan direction of $-0.2 \mathrm{~V}$ to $0.8 \mathrm{~V}$ indicates the stripping of $\mathrm{Pb}$ from the nanoporous Au electrode. By integrating the current for the formation or stripping of the $\mathrm{Pb}$ monolayer from the cyclic voltammogram, the $\mathrm{SA}$ of the nanoporous $\mathrm{Au}$ electrode could be determined. 16

Figure 1. 3. Schematic representation of the principle involved in the determination of SA (SA) by the ligand adsorption method. The ligands are adsorbed on the NPs and the SA of the NPs is determined from the difference between the amount of ligands in the fresh solution compared to that of the one with NPs

Figure 1. 4. Schematic representation of the principle involved in the determination of SA by the BET adsorption method. 18

Figure 1.5. Schematic representation of the principle involved in the determination of SA/V of Au NPs for determining their average size. 25 
Figure 1. 6. Schematic representation of the steps for the determination of SA/V of different aggregated states of Au NPs. The aggregates of Au NPs are formed after addition of acid (perchloric acid), which causes the neutralization of citrate group on the NPs. The aggregated NPs are then assembled on glass/ITO to measure SA/V. 27

Figure 1. 7. Schematic representation of different assembly methods of NPs on electrode surfaces. 29

Figure 1. 8. Schematic representation of selective dealloying of core shell/mixed alloys of AuAg system for formation of porous $\mathrm{Au}$ nanostructures and determination of SA/V of the porous Au nanostructures. The AuAg alloys or core shell nanostructures are formed by chemical synthesis, assembled on electrode surfaces, and dealloyed to remove Ag from them (Step I). After this, the SA/V of the $\mathrm{Au}$ nanostructures remaining on the electrode surface is determined

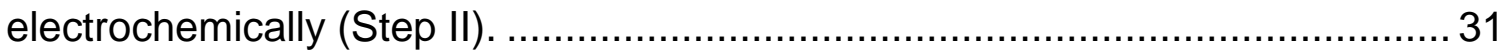

Figure 2. 1. General scheme of the experimental procedures involved in this dissertation.34

Figure 2. 2. Procedure involved in the synthesis of $4 \mathrm{~nm}$ diameter citrate-coated Au NPs.

Figure 2. 3. Procedure for the synthesis of $15 \mathrm{~nm}$ Au NSs by the citrate reduction method. 38

Figure 2. 4. Procedure for the seed-mediated growth of citrate-coated Au NPs by $\mathrm{H}_{2} \mathrm{O}_{2}$ reduction 39

Figure 2. 5. Attachment of Au NSs to glass/ITO through (a) APTES and (b) drop cast deposition

Figure 2. 6. Scheme showing the set-up for the electrophoretic deposition (EPD) of Au NSs on glass/ITO. 
Figure 2. 7. Illustration of the electrochemical set up.

Figure 2. 8. Important parts of the anodic stripping voltammogram of Au NSs attached to glass/ITO.

Figure 2. 9. Cyclic Voltammogram in $0.1 \mathrm{M} \mathrm{HClO}_{4}$ of $15 \mathrm{~nm} \mathrm{Au} \mathrm{NSs} \mathrm{attached} \mathrm{to}$ glass/ITO. 48

Figure 2. 10. Typical chronocoulometric curve for electrodeposition of Au NSs 49 Figure 2. 11. UV-vis spectroscopy of $15 \mathrm{~nm}$ Au NSs 51

Figure 2. 12. Illustration of the working principle of SEM (top) and a typical SEM image of $50 \mathrm{~nm}$ Au NSs attached to the surface of a glass/ITO electrode (bottom).

Figure 3. 1. Scheme for general experimental procedure followed in this work..58 Figure 3. 2. UV-Vis spectra (A) and SEM images (B-F) of $4.1 \pm 0.7,15.1 \pm 1.8$, $31.2 \pm 1.9,50.1 \pm 3.0$ and $70.8 \pm 2.7 \mathrm{~nm}$ citrate coated Au nanospheres. 60

Figure 3. 3. (A) Cyclic voltammograms of glass/ITO/APTES electrodes coated with $4 \mathrm{~nm}$ (red) and $15 \mathrm{~nm}$ (blue) Au NSs in $0.1 \mathrm{M} \mathrm{HClO}_{4}$ showing similar SA based on the $\mathrm{Au}_{2} \mathrm{O}_{3}$ reduction peak near $0.8 \mathrm{~V}$. (B) Anodic stripping voltammograms of the same glass/ITO/APTES electrodes in (A) coated with $4 \mathrm{~nm}$ (red) and $15 \mathrm{~nm}$ (blue) Au NSs in $0.01 \mathrm{M} \mathrm{KBr}$ plus $0.1 \mathrm{M} \mathrm{KClO}_{4}$. (C) Cyclic voltammograms of glass/ITO/APTES coated with $50 \mathrm{~nm}$ Au NSs obtained before (blue) and after (red) ozone treatment. We conducted the same type of SA/V and corresponding size analysis for all of the NS sizes in a similar way for each size. The results from 4 trials of each size are provided in Table 3.1 62

Figure 3. 4. Cyclic voltammograms of glass/ITO/APTES coated with $4 \mathrm{~nm}(\mathrm{~A})$ and $15 \mathrm{~nm}$ (B) Au NSs obtained before (blue) and after (red) ozone treatment. 68 
Figure 3. 5. Plot of experimentally-measured $S A / V$ as a function of $1 /$ radius as measured by SEM for 15, 32, 50 and $70 \mathrm{~nm}$ diameter citrate-coated Au NPs. The SA was measured in $0.1 \mathrm{M} \mathrm{HClO}_{4}$ and $\mathrm{V}$ was measured in $0.01 \mathrm{M} \mathrm{KBr}$ in $0.1 \mathrm{M}$ $\mathrm{KClO}_{4}$ without ozone treatment (blue) and with ozone treatment (red). 70

Figure 3. 6. Effect of coverage on SA/V for $4 \mathrm{~nm}$ (red) and $15 \mathrm{~nm}$ (black) Au NPs.

Figure 4. 1. Cyclic voltammograms (A) and linear sweep stripping voltammograms $(B)$ of non-aggregated (blue plots) and aggregated (orange plots) $4 \mathrm{~nm}$ diameter Au NPs attached to glass/ITO/APTES electrodes. The area under the peak marked with $\mathrm{a}^{*}$ in the cyclic voltammograms is proportional to the SA of the Au NPs while the area under the peak marked with a \# in the linear sweep stripping voltammogram is proportional to the volume of the Au NPs. Clearly the SA-to-volume ratio is significantly smaller for the aggregated $4 \mathrm{~nm}$ diameter $\mathrm{Au}$ NPs. .78

Figure 4. 2. Cyclic voltammograms (A) and linear sweep stripping voltammograms $(B)$ of non-aggregated (blue plots) and aggregated (orange plots) $15 \mathrm{~nm}$ diameter Au NPs attached to glass/ITO/APTES electrodes. The area under the peak marked with a * in the cyclic voltammograms is proportional to the SA of the Au NPs while the area under the peak marked with a \# in the linear sweep stripping voltammogram is proportional to the volume of the Au NPs. Clearly the SA-to-volume ratio is significantly smaller for the aggregated $15 \mathrm{~nm}$ diameter $\mathrm{Au}$ NPs 80 
Figure 5. 1. UV-Vis spectra (A) and TEM image (B) of $1.6 \pm 0.4 \mathrm{~nm}$ THPC Au NPs and SEM images (C-G) of $4.1 \pm 0.7,15.1 \pm 1.8,31.2 \pm 1.9,50.1 \pm 3.0$, and $70.8 \pm$ $2.7 \mathrm{~nm}$ of citrate-coated Au nanospheres (NSs) ............................ 90

Figure 5. 2. ASVs of $1.6 \mathrm{~nm}(\mathrm{~A}), 4 \mathrm{~nm}(\mathrm{~B})$ and $15 \mathrm{~nm}(\mathrm{C})$ diameter Au NSs assembled onto glass/ITO electrodes through APTES linker (red), by electrophoretic deposition (blue), and by drop-cast deposition (black). ASV was performed in $10 \mathrm{mM} \mathrm{KBr}$ plus $0.1 \mathrm{M} \mathrm{KClO}_{4}$ solution with a scan rate of 0.01 $\mathrm{V} / \mathrm{s}$ 91

Figure 5. 3. CVs (A) and ASVs (B) and SEM images (C to E) of $15 \mathrm{~nm}$ Au NSs assembled to glass/ITO electrode by different assembly methods. CVs were performed in $0.1 \mathrm{M} \mathrm{HClO}_{4}$ and $\mathrm{ASV}$ s performed in $10 \mathrm{mM} \mathrm{KBr}$ plus $0.1 \mathrm{M} \mathrm{KClO}_{4}$ solution with a scan rate of $0.01 \mathrm{~V} / \mathrm{s}$. 95

Figure 5. 7. Schematic illustration of explanation for differences in SA/V of Au NSs for different assembly methods. 109

Figure 6. 1. UV vis spectra (A) and SEM images of Au NSs (B) and Au50Ag50, $\mathrm{Au}_{25} \mathrm{Ag}_{75}$ and $\mathrm{Au}_{10} \mathrm{Ag}_{90}(\mathrm{C}$ to $\mathrm{E}$ ) alloys by modification in the method by $\mathrm{El}$ Sayed....... 120

Figure 6. 2. Scheme for general experimental work involved in this work 121

Figure 6. 3. ASVs of $50 \mathrm{~nm} \mathrm{Ag} \mathrm{NPs} \mathrm{attached} \mathrm{to} \mathrm{glass/ITO/APTES} \mathrm{without} \mathrm{further}$ treatment (red plot) and after soaking in $200 \mathrm{mM} \mathrm{Fe}\left(\mathrm{NO}_{3}\right)_{3} \cdot 9 \mathrm{H}_{2} \mathrm{O}$ for 3 hours (blue plot). 123 
Figure 6. 4. CVs (top) and ASVs (bottom) of $\sim 50 \mathrm{~nm}$ diameter pure Au NSs (blue plots) and nanoporous $\mathrm{Au}$ NSs prepared by dealloying the $\mathrm{Ag}$ from $\mathrm{Au}_{50} \mathrm{Ag}_{50}$ (green plots), $\mathrm{Au}_{25} \mathrm{Ag}_{75}$ (red plots), and $\mathrm{Au}_{10} \mathrm{Agg}_{90}$ (black plots). 125

Figure 6. 5. SEM images of $A_{450} A_{50}$ alloy NPs $(A, B), A_{75} A_{25}(C, D)$, and $\operatorname{Au}_{10} \mathrm{Ag}_{90}(\mathrm{E}, \mathrm{F})$ before $(\mathrm{A}, \mathrm{C}, \mathrm{E})$ and after $(\mathrm{B}, \mathrm{D}, \mathrm{F})$ dealloying and the corresponding histograms $(\mathrm{G}, \mathrm{H}, \mathrm{I})$. The histograms show the number of intact Au NPs and number of porous/flattened/distorted NPs for each size range based on the SEM

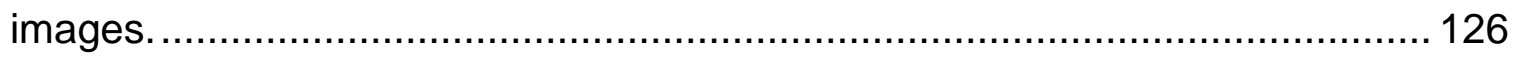

Figure 6. 6. SEM images of individual $A u_{25} A_{75}$ alloy NSs (A and B) and Au NSs obtained after dealloying $(\mathrm{C}$ to $\mathrm{H})$ 128

Figure 6. 7. UV vis spectra of $\mathrm{Au}_{25} \mathrm{Ag}_{75}$ alloys on glass/ITO before dealloying (blue spectra) and after dealloying (red spectra) 131

Figure 6. 8. Plots of $E_{p}(A)$ and $S A / V(B)$ vs vs $\%$ of Ag dealloyed from the different AuAg alloy NSs. 132

Figure 6. 9. Plot of $S A$-to-volume ratio $(S A / V)$ and oxidation peak potential (Ep) for Au NSs obtained by dealloying AuAg alloys having different percentatges of $\mathrm{Ag}$ in them 133 


\section{LIST OF TABLES}

Table 3. 1. SEM measured radius, average electrochemically-measured SA/V, and calculated radius from SA/V for different sizes of Au NSs with and without ozone treatment of the sample

Table 3. 2. SEM measured radius of the nanospheres (NSs) (average \pm std. dev.), charge under the oxide reduction peak and the charge under the anodic stripping peaks, SA/V (average \pm std. dev.) and calculated radius from SA $/ V$ for different sized Au NSs without ozone treatment. 63

Table 3. 2. SEM measured radius of the nanospheres (NSs) (average \pm std. dev.), charge under the oxide reduction peak and the charge under the anodic stripping peaks, SA/V (average \pm std. dev.) and calculated radius from SA $/ V$ for different sized Au NSs without ozone treatment. 64

Table 3. 2. SEM measured radius of the nanospheres (NSs) (average \pm std. dev.), charge under the oxide reduction peak and the charge under the anodic stripping peaks, SA/V (average \pm std. dev.) and calculated radius from SA $/ V$ for different sized Au NSs without ozone treatment. 64

Table 3. 3. SEM measured radius of the NSs (average \pm std. dev.), charge under the oxide reduction peak and the charge under the anodic stripping peaks, SA/V (average \pm std. dev.) and calculated radius from SA/V for different sized Au NSs

with ozone treatment. 65 
Table 3. 3. SEM measured radius of the NSs (average \pm std. dev.), charge under the oxide reduction peak and the charge under the anodic stripping peaks, SA $/ \mathrm{V}$ (average \pm std. dev.) and calculated radius from SA/V for different sized Au NSs with ozone treatment. 66

Table 3. 3. SEM measured radius of the NSs (average \pm std. dev.), charge under the oxide reduction peak and the charge under the anodic stripping peaks, SA/V (average \pm std. dev.) and calculated radius from SA/V for different sized Au NSs

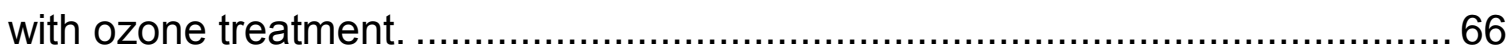

Table 3. 4. Effect of coverage on SA/V for $4 \mathrm{~nm}$ and $15 \mathrm{~nm}$ Au NPs............... 72 Table 3. 5. SA (charge under the oxide reduction peak) and total volume (charge under the anodic stripping peaks), SA/V (average \pm std. dev.) at different coverages $\left(10^{-4}, 10^{-5}\right.$ and $\left.10^{-6}\right)$ for $4 \mathrm{~nm}$ and $15 \mathrm{~nm}$ Au NSs. ........................ 73

Table 4. 1. Electrochemical SA-to-volume ratio measurements for non-aggregated and aggregated $4 \mathrm{~nm}$ diameter and $15 \mathrm{~nm}$ diameter $\mathrm{Au}$ NPs 81

Table 5. 1. SEM/TEM measured radius, average electrochemically-measured $\mathrm{SA} / \mathrm{V}$, and average $\mathrm{E}_{\mathrm{p}, \mathrm{ox}}$ of Au NSs for different assembly methods. All standard deviations represent one sigma value $(n=3)$ 96

Table 5. 2. Charge under the oxide reduction peak (CV), charge under the anodic stripping peaks (ASV), SA/V, average SA/V ( \pm std. dev.), oxidation peak potentials $\left(E_{p, o x}\right)$, and average oxidation peak potentials ( \pm std. dev.) as a function of NS size and assembly method (with ozone treatment for all sizes except $1.6 \mathrm{~nm} \mathrm{Au}$

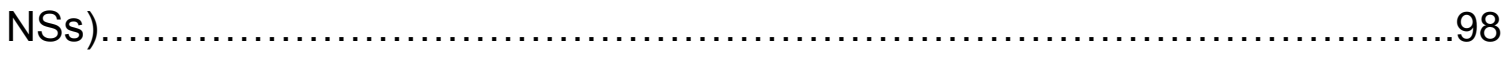


Table 5. 3. Charge under the oxide reduction peak (CV), charge under the anodic stripping peak (ASV), peak oxidation potential ( $\left.E_{p, o x}\right)$, and average peak oxidation potentials ( \pm std. dev.) for $1.6 \mathrm{~nm}$ Au NSs assembled by APTES linking and direct soaking in ITO. 102

Table 5. 4. Size of Au NSs, average electrochemically-measured SA/V for drop cast deposited with $0.28 \% \mathrm{w} / \mathrm{v}, 0.072 \% \mathrm{w} / \mathrm{v}$ and $0.036 \% \mathrm{w} / \mathrm{v}$ of $\mathrm{C}$ black with $\mathrm{Au}$ NSs and average $E_{p, o x}$ for drop cast deposited with $0.28 \% \mathrm{w} / \mathrm{v}, 0.072 \% \mathrm{w} / \mathrm{v}$ and $0.036 \% \mathrm{w} / \mathrm{v}$ of $\mathrm{C}$ black with Au NSs. All standard deviations represent one sigma value $(n=3)$. 106

Table 6. 1. SEM determined outer diameter (ave \pm std dev) of Au and AuAg alloy NSs before and after dealloying AuAg alloys, SA/V (ave \pm std dev) and $E_{p}(a v e \pm$ std dev) of the pure Au NSs and AuAg alloy NSs after dealloying. 129

Table 6. 2. Au NSs type, CV measured SA, ASV measured volume, SA-to-volume ratio (SA/V), average $S A / V \pm$ std. dev., oxidation peak potential $\left(E_{p}\right)$ and average Ep \pm std. dev. 130 


\section{CHAPTER I \\ INTRODUCTION}

\subsection{Main Goal/Summary}

The main goal of this work was to develop an electrochemical approach for measuring the surface area-to-volume ratio $(\mathrm{SA} / \mathrm{V})$ of electrode-attached nanostructures in order to analyze their size, aggregation, and nanoporosity. Using this approach, we directly determined the size of Au nanospheres (NSs) from $4 \mathrm{~nm}$ to $70 \mathrm{~nm}$ in diameter. Also, we measured the SA/V of nanoporous Au NPs that were prepared by dealloying Ag from AuAg alloy NSs. In addition to learning about the porosity as a function of $\mathrm{Ag}$ percent composition prior to dealloying, this work provided physical insight about the relationship between metal oxidation and the $S A / V$, especially for irregular nanostructure geometries. Finally, we used the SA/V measurement to characterize the activity and oxidation properties of Au NSs assembled by different methods and on different electrode materials. This provides a means to optimize the assembly of metal NSs for electrocatalysis and other electrochemical applications in terms of their activity and stability. It also physically provides a correlation between the metal NS oxidation potential and SA/V. Chapter I describes background information on metal NPs and their commonly used characterization methods as a comparison to the electrochemical methods used in this work. It also reviews theories on size-dependent metal NP oxidation and recent work on nanoporous particles and their applications. Chapter 
II provides the experimental methods and instrumentation used to complete the research described in later chapters of this dissertation. Chapter III is focused on the electrochemical determination of the SA/V of Au nanospheres and use of SA/V to analyze the size of Au NSs. Chapter IV describes the SA/V of different aggregated states of Au NSs. Chapter V describes the impact of different assembly methods of Au NSs on their electrochemical SA/V and stability. Chapter VI is focused on the determination of the SA/V of flattened, distorted and porous $\mathrm{Au}$ nanostructures formed by dealloying of AuAg alloy NSs. Chapter VII summarizes the findings of all of the projects and suggests future directions.

\subsection{Motivation/Objective}

The motivation behind this research was to determine the SA/V of metal NPs, specifically Au NSs. Surface attached metal NSs are widely used for a variety of applications, such as catalysis, sensing and energy storage. There are methods available for measuring the SA of these surface-attached metal NSs in order to correlate it to their activities. However, it is not very useful to know only the SA without the volume. It is more informative to normalize the SA to volume because the volume is related to the overall amount of material being used for an application and it is related to the overall cost of that material as well. Ideally, it is important to have a lot of SA in order to exploit surface reactivity for catalysis and sensing, but without using a lot of volume. Therefore, it is necessary to know SA/V.

Another important reason to know SA/V is in the case of spherical shaped NPs, where the SA/V is related to their radius by the relation $S A / V=3 / r$, where $r$ 
is the radius of the nanoparticle. Using this relation, the radius of NSs can be determined. Many properties of metal NSs, such as catalysis and sensing, are related to their size. Thus, determining the size of metal NSs based on SA/V can be useful to compare the effect of their size on their electrocatalytic activity, especially when comparing different particles and approaches for electrocatalysis. $\mathrm{SA} / \mathrm{V}$ determination for various irregular or porous nanostructures can be useful to correlate their SA/V to their electrocatalytic or sensing properties as well.

For their varied applications and in a physical sense, the stability of metal NPs is very important. The stability of metal NPs is related to their oxidation potential, which is usually correlated to either their size or curvature in the literature. Our work correlates the oxidation potential with SA/V, which is especially useful for irregular structures where there is no real clear size definition. We can also explain the change in oxidation behavior of metal NPs based on SA/V even when sizes or their curvature does not change. This includes the assembly of metal NPs by different assembly methods and the oxidation of aggregated and porous nanostructures. Normalizing the SA to the volume to determine SA/V of metal NPS of different size, aggregation state, irregular or porous nature and assembled by different methods can help to correlate their stability, activity and reactivity to SA/V.

\subsection{Applications of Metal NPs}

Metal NPs (NPs) have gained tremendous interest recently due to their wide applications in catalysis, ${ }^{1-4}$ sensing, ${ }^{5-6}$ drug delivery, ${ }^{7-8}$ and energy storage ${ }^{9-14}$ They are of special interest compared to their bulk analogues. This is because as 
the bulk metals are converted to metal nanoparticle there is an increase in overall SA (SA) for reactions to take place relative to the total amount of material being used (or volume), which allows higher reactivity with lower cost. In addition, there is a large increase in the number of specific types of atoms on the NP surface as the size is reduced. For example, more corner and edge sites (relative to the total volume of material) become exposed with decreasing NP size. The number of these sites grows exponentially with the reduction in NP size and these corner and edge sites are often the reactive sites for catalytic reactions. ${ }^{15-16}$ This can lead to a significant increase in activity with a decrease in NP size. These properties make metal NPs important for a number of applications, such as therapeutics and catalysis.

Sil ${ }^{17}$ used Pd NPs in the size range of $5-20 \mathrm{~nm}$ as a sensing layer on the surface acoustic wave (SAW) device to detect $\mathrm{H}_{2}$ gas. As the $\mathrm{H}_{2}$ gas interacts with the Pd NP film the added mass modifies the acoustic wave propagation, which allowed detection of the gas. Huang and coworkers ${ }^{18}$ developed an immunosensor using an assembly of N-aminobutyl-N-ethylisoluminol-functionalized Au NPs with antibody, bovine serum albumin (BSA) and $\mathrm{Co}^{2+}$. These multifunctionalized $\mathrm{Au}$ NPs showed excellent chemiluminescent (CL), catalytic and immunogenic activity. Mistry and coworkers ${ }^{19}$ studied the size-dependent catalytic activity of micellesynthesized Au NPs in the 1-8 nm size range for electroreduction of $\mathrm{CO}_{2}$ to $\mathrm{CO}$ in $0.1 \mathrm{M} \mathrm{KHCO}_{3}$. They observed a dramatic increase in current density with a decrease in NP size and a decrease in Faradaic selectivity towards CO formation. They found using DFT calculations that these results are due to an increase in the 
number of low-coordinated sites on small NPs that favors evolution of $\mathrm{H}_{2}$ over $\mathrm{CO}_{2}$ reduction to $\mathrm{CO}$. Zhao and coworkers ${ }^{20}$ sandwiched Pt NPs between an inner core and an outer shell composed of a metal organic framework (MOF) having metal nodes of $\mathrm{Fe}^{3+}, \mathrm{Cr}^{3+}$ or both. These acted as good catalysts that could convert a range of $\alpha, \beta$-unsaturated aldehydes with high efficiency and showed enhanced selectivity towards unsaturated alcohols. Different shapes of metal NPs, such as cubes, rods, and plates, are also used for sensing, imaging and drug delivery. Baciu and group ${ }^{21}$ used lipid layer coated Au nanorods (NRs) as a sensing platform for monitoring the interactions between proteins and biological membranes. The sensing occurred on these membrane-covered Au NRs by monitoring spectral shifts in fast single particle spectroscopy (fastSPS). Ali and coworkers $^{22}$ developed a drug delivery platform using Au NRs conjugated to rifampicin (RF), an antituberculotic drug, to deliver it into macrophage cells. The nature of the macrophage cells allowed the NPs to actively internalize in them and release the RF after uptake, validating the usefulness of Au NRs to deliver rifampicin (RF). Amjadi and coworkers ${ }^{23}$ developed a plasmonic probe for the detection of trace amounts of $\mathrm{Se}(\mathrm{IV})$ by monitoring morphological transformations of $\mathrm{Ag}$ nanoprisms (NPRs). The surface plasmon resonance (SPR) of Ag NPRs blue-shifted from $720 \mathrm{~nm}$ to lower wavelength values of $550 \mathrm{~nm}$ in the presence of $\mathrm{Se}(\mathrm{IV})$. The shift is due to etching of the corners of the NPRs in the presence of selenite ions, which acts as a method for sensing them. These few examples out of the many in the literature clearly demonstrate the use of various metal NPs for 
catalysis, gas sensing, drug delivery, biosensing, and trace metal detection as evidence of their importance.

The composition of metal NPs are also often tuned for different applications, especially in catalysis. The atomic arrangement of multimetal NPs can be tuned from core/shell to various alloy forms for different applications. Kilic and coworkers ${ }^{24}$ synthesized Ni@Pd core@shell NPs using a one pot reaction with successive reduction of $\mathrm{Ni}$ and $\mathrm{Pd}$ metal precursors in the presence of oleylamine and trioctyl phosphine and assembled these core@shell NPs on reduced graphene oxide, finding them to be highly efficient for $\mathrm{C}-\mathrm{H}$ bond arylation reactions. Feng and coworkers ${ }^{25}$ synthesized Cu-Zn alloy NP catalysts by laser ablation in liquid and found that the alloy NPs exhibited excellent selectivity for $\mathrm{C}_{2} \mathrm{H}_{4}$ formation in $\mathrm{CO}_{2}$ electroreduction, having high faradic efficiency of $33.3 \%$ at $-1.1 \mathrm{~V}$ (vs RHE). The high catalytic activity was attributed to the proximity of $\mathrm{Cu}$ and $\mathrm{Zn}$ atoms in the alloy system that facilitates both stabilization of the $\mathrm{CO}^{*}$ intermediate and transfer from $\mathrm{Zn}$ atoms to $\mathrm{Cu}$ atoms, where dimerization and protonation occurs to give high yields of ethylene.

\subsection{Characterization Methods for Metal NPs}

With all the obvious advantages and useful applications, metal NPs are characterized by different methods to correlate their properties for their different applications. There are different techniques available for the characterization of metal NPs, and the most common classes include electron microscopic, optical spectroscopic and mass spectrometric methods. 
1.4.1. Electron and scanning probe microscopy methods. Electron microscopic techniques involve the interaction of electrons with the specimen under study while scanning probe techniques involve the use of a probe tip to image the sample surface in order to analyze different properties, such as size, composition, aggregation and morphology of NPs. Different electron microscopic techniques, such as Scanning Electron Microscopy (SEM), 26-27 Transmission Electron Microscopy (TEM), ${ }^{28-30}$ Scanning Transmission Electron Microscopy (STEM) $^{31-33}$ and scanning probe techniques such as Scanning Tunneling Microscopy (STM) ${ }^{34-36}$ and Atomic Force Microscopy (AFM), ${ }^{37-38}$ are being used to characterize metal NPs.

In SEM, a focused beam of electrons is used to scan the surface of a sample. These electrons interact with atoms in the sample which produces secondary and backscattered electrons that contain information about the surface topography of a sample. SEM provides useful information on morphology, ${ }^{39-40}$ size $^{41-42}$ and aggregation ${ }^{43}$ of NPs.

In the case of TEM, a beam of electrons transmitting through a sample specimen interacts with the sample and forms an image of the sample onto a detector. ${ }^{44}$ TEM analysis provides useful information about morphology ${ }^{45}$ and size $^{45}$ of NPs.

STEM involves a highly focused electron probe used to scan over the material under study, where the collection of transmitted electrons is measured as a function of probe position. The transmitted electrons at a high scattering angle 
forms a high-resolution image of the sample that reveals information about morphology and size of NPs. ${ }^{46}$

1.4.2. Optical spectroscopy methods. Optical spectroscopic methods include the interaction of light with matter gives information about the size, compositional and structural properties of NPs. The commonly used optical spectroscopic methods are UV-Vis, ${ }^{47}$ Localized Surface Plasmon Resonance $\left(\right.$ LSPR) ${ }^{48}$ and Dynamic Light Scattering (DLS). ${ }^{49}$

In UV-vis spectroscopy, when the energy of the light source matches the energy required for an electronic transition within the molecule, absorption of light in the UV-vis region occurs. Part of this light intensity is absorbed as the electron is promoted to a higher energy orbital. A spectrophotometer records the wavelength at which the absorption of light occurs, and the degree of absorption at each wavelength. In the case of NPs, this phenomenon is related to the size and concentration ${ }^{49}$ of NPs and has been used to study interesting phenomena such as Ostwald ripening in NPs in which small particles dissolve and redeposit on the larger particles to form larger particles. ${ }^{50}$ The UV-vis spectroscopy can monitor this growth of NPs. In the case of UV-vis spectroscopy, the localized collection of electrons within the metal NPs can be excited by light in the UV-vis range when the frequency of light matches the resonance frequency of the collective oscillating electrons. This is known as localized surface plasmon resonance (LSPR). 
Localized Surface Plasmon Resonance (LSPR) is the result of resonance caused by the interaction of light with the localized surface plasmons in metal NPs. The plasmon absorption bands are characteristic of the type of material and are highly sensitive to the composition, size and shape of the nanostructures. ${ }^{51}$ Besides size and shape it is also useful to monitor some aspects of NPs such as agglomeration. 52

Dynamic light scattering is based on fluctuations in scattering intensity due to random movement of NPs by Brownian motion in their suspension. ${ }^{53}$ The fluctuations in scattering intensity are a function of NP size and also allow one to study important properties in NPs such as aggregation behavior ${ }^{54}$ and for analysis of the interactions of biomolecules with NPs. ${ }^{55}$

\subsubsection{Electrochemical methods for NP analysis. Electrochemical} methods offer benefits such as low cost, simple operation, speed of analysis, and high throughput. Electrochemical methods are being used extensively to analyze NPs and/or properties associated with them. Some of the electrochemical methods for analyzing NPs include Scanning Electrochemical Microscopy (SECM), electrocatalysis, coulometry (anodic and cathodic), resistive pulse sensing, metal NP charging, electrophoretic separation, and anodic stripping voltammetry (ASV).

1.4.3.1. Electrocatalysis. The electrocatalytic method involves analyzing metal NPs indirectly by measuring the electrocatalytic oxidation or reduction of molecules interacting at the surface of the NPs in contact with an ultramicroelectrode. Some examples include electrooxidation of $\mathrm{N}_{2} \mathrm{H}_{4}$ at the 
surface of platinum NPs (Pt NPs) ${ }^{56}$ and that of $\mathrm{H}_{2} \mathrm{O}_{2}$ at the surface of ruthenium oxide (RuOx) NPs. ${ }^{57}$ The recorded current time (i-t) transients obtained from the individual NP impacts on the microelectrode provide information about the size and interaction of the NPs with the substrate (elastic collision and adsorption) as well as the kinetics of the electrochemical reaction on the surface of the NPs.

1.4.3.2. Capacitive Charging. A size-dependent quantized double layer (QDL) charging effect occurs during capacitive charging of monolayer-protected $\mathrm{Au}$ and $\mathrm{Ag}$ nanostructures (MP-AgNSs). ${ }^{58} \mathrm{~A}$ size-dependent magnitude of the surface charge for $\mathrm{Si} \mathrm{NSs}^{59}$ that increased with an increase in particle size has been successfully employed for NP characterization.

1.4.3.3. Single Particle Sizing. Electrochemical sizing of single NPs has been accomplished by monitoring an ionic current drop in the i-t trace as NPs translocate across a nanopore channel. This translocation through a nanopore has been used for analyzing single $\mathrm{Au}^{60}$ and silica NSs. ${ }^{61}$

\subsubsection{Scanning Electrochemical Microscopy (SECM). SECM} involves the measurement of Faradaic electrochemical current at an ultramicroelectrode when it is held or moved in a solution above a surface of interest. The surface perturbs the electrochemical response of the tip, thereby providing information about the nature and properties of the surface. SECM has been successfully used for the analysis of $\mathrm{Ag}^{62}{ }^{6 t ~ N P s}{ }^{63}$ and studying some catalytic reactions on the surface of these NPs, monitoring electron transfer kinetics at nano sized electrodes. 


\subsubsection{Electrochemical Scanning Tunneling Microscopy (EC-STM).}

The EC-STM is a STM that measures the structure and morphology of electrode surfaces or atomic or molecular adsorbates on the electrode at atomic or molecular scales while the electrode is under potential control in an electrochemical cell. It has been used for analyzing the adsorption of molecules ${ }^{64}$ and corrosion behavior of different metal NP surfaces. ${ }^{65}$

\subsubsection{Scanning Electrochemical Cell Microscopy (SECCM). SECCM} consists of a dual-barrel theta pipet (a type of double-channel pipette having inlet and outlet channels for the electrolyte) probe containing electrolyte solution and a quasi-reference counter electrodes (QRCE) in each barrel. This is a highresolution electrochemical scanning probe technique. Specifically, a thin layer of electrolyte protruding from the tip of the pipet ensures a gentle contact of the meniscus with a surface. The combination of SECCM with other microscopic techniques, like scanning electron microscopy (SEM), allows one to relate the current-voltage characteristics to the spatial position, providing useful information about surface structure. Some useful observations using SECCM include the study of the interactions between NPs and surface-modified electrodes ${ }^{66}$ and imaging of electro-oxidation on the electrode surface. ${ }^{67}$

\subsubsection{Electrochemical Atomic Force Microscopy (EC-AFM).} Electrochemical AFM (EC-AFM) is type of Scanning Probe Microscopy (SPM), which consists of Atomic Force Microscopy (AFM) combined with electrochemical measurements. This technique allows to perform in-situ AFM measurements in 
an electrochemical cell to monitor actual changes occurring in the electrode surface morphology at the time of electrochemical reactions. Using EC-AFM solid liquid interphase can be analyzed. ${ }^{68}$ The method allows to fabricate metal and semiconductor nanostructures on the working electrode (WE) and also to perform and study the electrodeposition of different metals such as copper on electrode surfaces. ${ }^{69}$

1.4.3.8. Scanning lon-Conductance Microscopy (SICM). With SICM an electrode inside a nanopipette tip is used as the probe, where a potential is applied between the internal electrode and a second electrode outside the probe tip. This leads to the flow of an ion current between the internal and external electrodes through the nanosized pipette opening while the pipette is scanned over a surface of interest. This allows an analysis of surface topography of micron and nanometer-sized structures in aqueous electrolytes. When the probe tip is brought from bulk solution to near the surface, the high precision and high resolution ionic current measurements provide information about the surface, which can be displayed as an image. SICM has been successfully used to visualize electrochemical reactivity at electrochemical interfaces ${ }^{70}$ and probe interactions of NPs with biological systems. ${ }^{71}$ 


\subsection{SA Measurements of NPs}

Metal NPs are used for a number of applications, such as catalysis, sensing, energy storage and therapeutics. For most applications, the exposed SA of the metal NPs plays a key role in their function. As metals change from their bulk form to NP form and decrease in size, their effective SA relative to their volume increases significantly, as there are an increasing number of surface atoms compared to the internal atoms. As shown in Figure 1.1 with the case of a cubic-

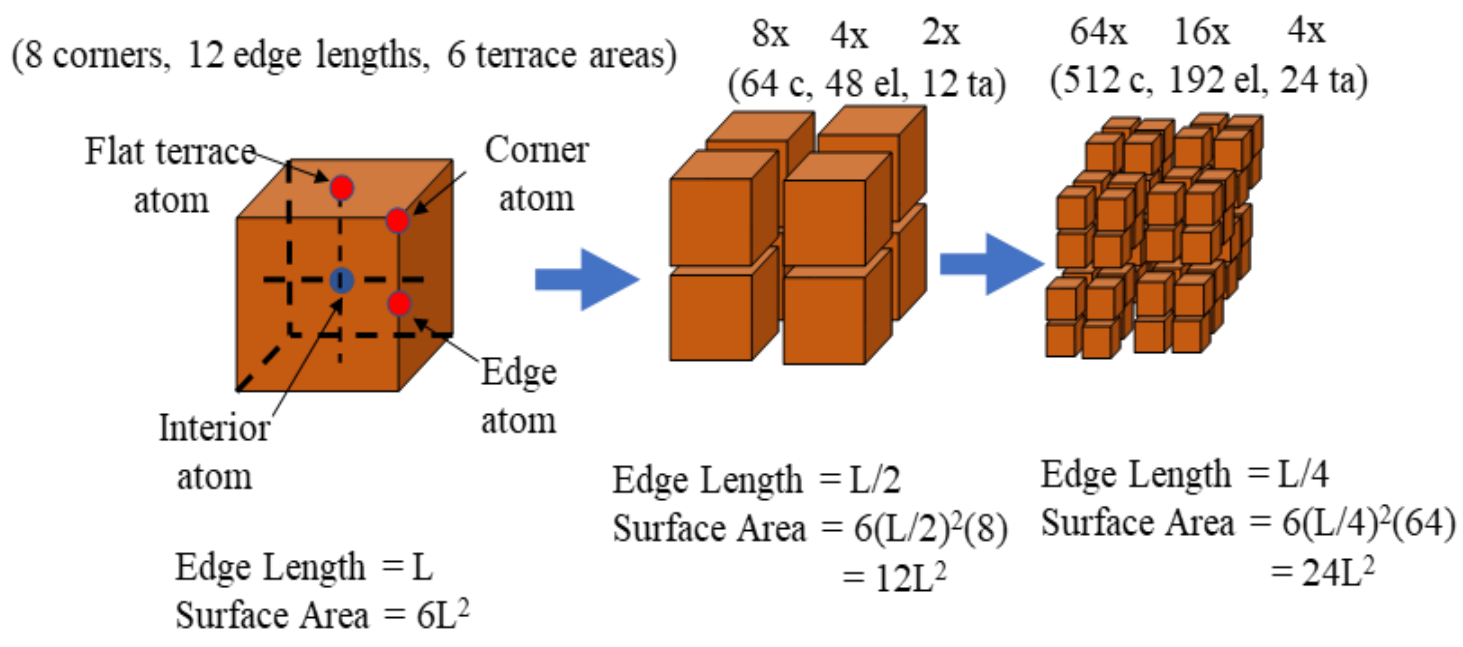

Figure 1. 1 Schematic representation showing the increase in SA (SA), edge lengths (el), corner (c), and terrace (ta) sites with a decrease in cube size (edge length, L) of NPs by breaking up 1 cube into 8 cubes and then into 64 cubes.

shaped structure, the number of surface atoms relative to the interior atoms 
increases as the larger individual cube with edge length $L$ is broken up in to 8 cubes and then into 64 cubes. This shows a simple inverse relationship between SA and the cube edge length, keeping the total volume constant. If the NP function, such as catalytic turnover rate, depended on total exposed SA, then breaking up the cube into cubes with edge length half of the original edge length should then double the performance. Interestingly, the number of total edge lengths and corner sites exposed as the cube is broken up does not follow this simple inverse relationship. As the single cube is broken up into 8 cubes, the SA doubles as mentioned but the amount of exposed edge length increases by 4 and the number of cube corners increases by 8 . If the function of the cubes depends on these particular sites, then the function would increase 4-fold and 8-fold, respectively, instead of just 2 -fold if it depended on SA only. This simple geometric consideration shows how the performance of a material can improve dramatically with decreasing size, not even considering other electronic effects of decreasing size. The total number of surface atoms and specific sites on the surface are the key to improving their performance as catalysts, energy storage materials, and therapeutic agents. Measuring the available SA is therefore very important. The commonly used methods for SA determination of metal NPs include underpotential deposition (UPD), ligand adsorption and the Brunauer-Emmett-Teller (BET) adsorption method 
1.5.1. Underpotential deposition (UPD). This is an electrochemical method in which electrodeposition of one metal by reduction of its cation on another metal occurs at a potential more positive than the equilibrium (Nernst) potential for the metal/metal ion pair of the depositing metal. ${ }^{72}$ This makes it possible to deposit 1 layer of metal onto another metal at the UPD potential. This can be exploited for SA measurements by depositing a monolayer of metal 2 by UPD onto metal 1 . The deposited metal 2 is then electrochemically removed by metal oxidation (stripped) and the charge of the stripping peak is proportional to the SA of metal 1 since metal 2 only formed 1 layer of metal over the surface of metal $1 .{ }^{73-74}$ As shown in Figure 1.2, Liu and coworkers ${ }^{75}$ determined the SA of Au by depositing a UPD layer of $\mathrm{Pb}$ onto it and by scanning the potential range from $0.5 \mathrm{~V}$ to $-0.05 \mathrm{~V}$.

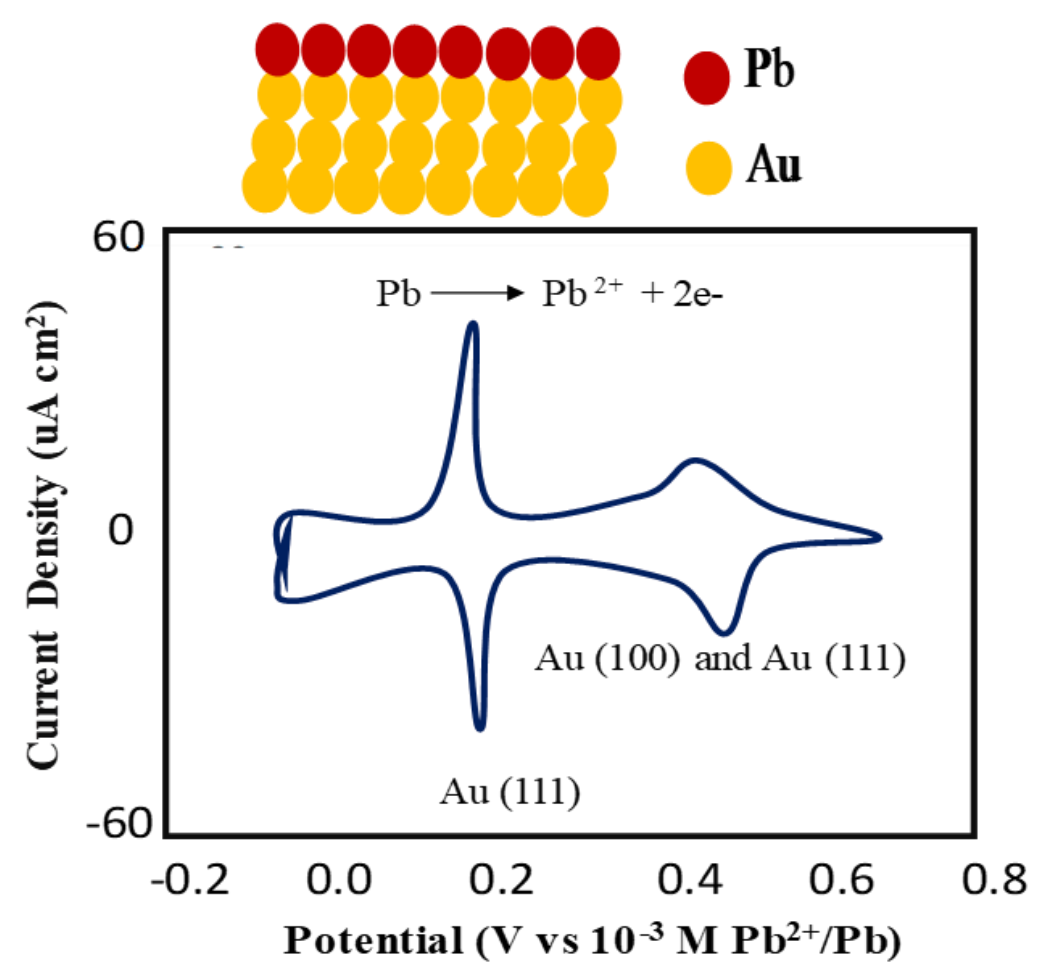


Figure 1. 2. Schematic representation of the principle involved in determination of SA (SA) by underpotential deposition (UPD). The peak at $0.190 \mathrm{~V}$ and $0.450 \mathrm{~V}$ in the scan direction from $0.8 \mathrm{~V}$ to $-0.2 \mathrm{~V}$ in cyclic voltammetry indicates the formation of $\mathrm{Pb}$ monolayer on the nanoporous $\mathrm{Au}$ electrode, while the same peaks in the scan direction of $-0.2 \mathrm{~V}$ to $0.8 \mathrm{~V}$ indicates the stripping of $\mathrm{Pb}$ from the nanoporous Au electrode. By integrating the current for the formation or stripping of the $\mathrm{Pb}$ monolayer from the cyclic voltammogram, the $\mathrm{SA}$ of the nanoporous $\mathrm{Au}$ electrode could be determined.

Two peaks appear in the voltammogram, which corresponds to UPD of Pb onto $A u(111)$ and $A u(100)$, showing that the UPD potential is sensitive to the crystal face of the Au as well. The total charge passed during the deposition or stripping of $\mathrm{Pb}$ (both peaks) is proportional to the SA of the Au. This approach has been used successfully to measure the SA of important metals, such as Platinum and Ruthenium. ${ }^{76}$

1.5.2. Ligand adsorption method. This method is also important for SA determination of NPs. The method is employed in the case of solution phase NPs. In this method the NPs in the solution phase whose SA is to be determined is mixed with a known quantity of a ligand that adsorbs on the NPs. For example, in the case of thiol ligands, the ligands bind to the NPs via Au-S interaction. After mixing a specified amount of Au NPs with a known quantity of ligands, the amount of ligands retained by the Au particles is determined from the difference between the amount of thiol in the fresh solution compared to that of the one with NPs. The 
methodology is demonstrated in Figure 1.3. This method has been successfully used for the determination of SA of Au NPs and can be employed for other metal NPs.

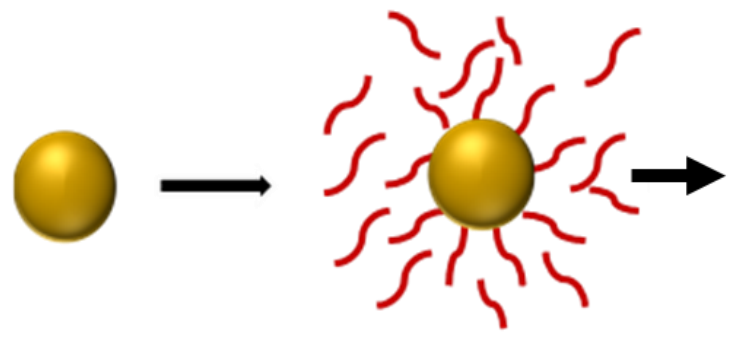

Nanoparticles Nanoparticles + Ligand
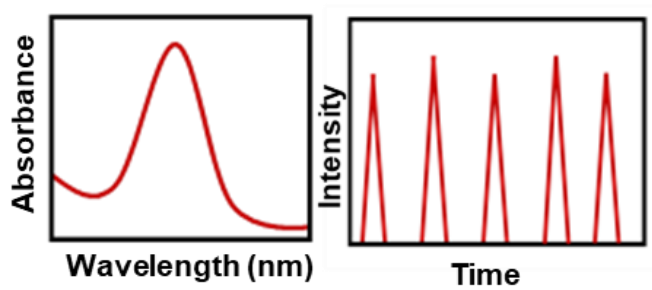

Quantification of adsorbed ligands by GC-MS or UV vis spectroscopy

Figure 1. 3. Schematic representation of the principle involved in the determination of SA (SA) by the ligand adsorption method. The ligands are adsorbed on the NPs and the SA of the NPs is determined from the difference between the amount of ligands in the fresh solution compared to that of the one with NPs

1.5.3. The Brunauer-Emmett-Teller (BET) adsorption method. This method for SA determination relies on the adsorption of gas molecules (adsorbate molecules) on the surface of the materials or the nanostructures whose SA is to be determined (adsorbent molecules) as shown in Figure 1.4.

$$
S_{t} \frac{W_{m} N A_{c s}}{M}
$$

where $S_{t}$ is total $S A, W_{m}$ is weight of monolayer, $N$ is Avogadro's number, $A_{c s}$ is adsorbate cross sectional area and $\mathrm{M}$ is the molecular weight of adsorbate. 

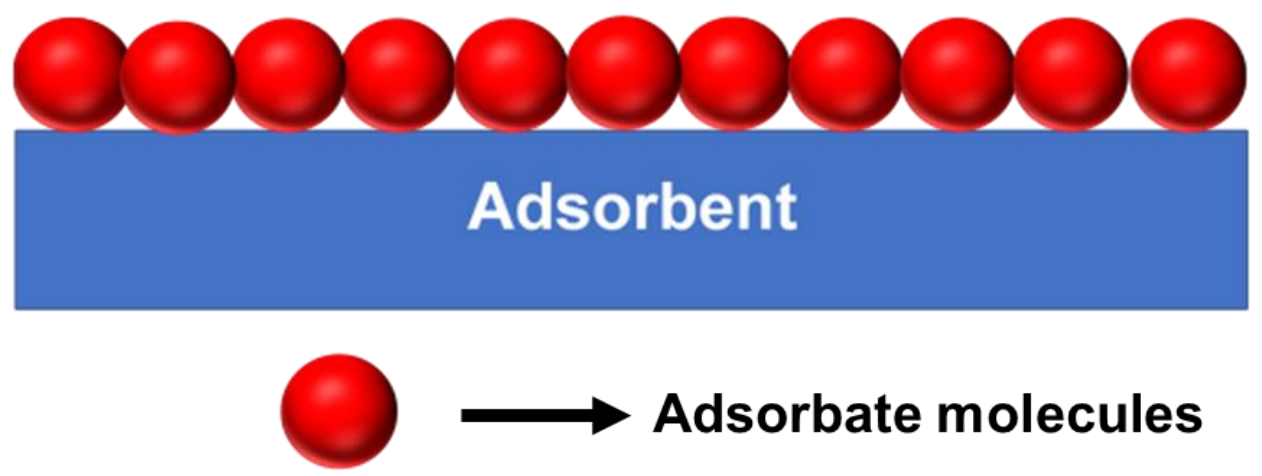

Figure 1. 4. Schematic representation of the principle involved in the determination of SA by the BET adsorption method.

In the end, besides the total SA, BET can give BET SA, which is the SA per mass or SA/V if you convert mass into volume using the density. The BET adsorption method has been used successfully for determining the SA of metal NPs. ${ }^{77-78}$

The method uses inert gases such as $\mathrm{N}_{2}$, which do not react with the material whose surface is to be determined. Another advantage of using $\mathrm{N}_{2}$ gas is its availability in highly pure form and strong interactions with the majority of materials. Usually, in order to overcome the problem of weak interaction of the gas with the materials, the surface is cooled using liquid $\mathrm{N}_{2}$ to obtain detectable amounts of adsorption. Typically, a specified amount of nitrogen gas is passed through a sample cell and a condition of relative pressure less than atmospheric pressure is achieved by creating a partial vacuum. There is no more adsorption after the saturation pressure. The sample material is removed from nitrogen atmosphere after the adsorption layer is formed and is heated to release the adsorbed nitrogen, which then is quantified. The data is observed in the form of a BET isotherm, which is a plot of the amount of gas adsorbed as a function of the 
relative pressure. The BET equation below uses the information from the adsorption isotherm to measure the SA of the sample.

\subsection{Size-Dependent Oxidation and Stability of Metal NPs}

The oxidation of metal NPs is a size dependent phenomenon. Earlier theoretical work on size dependent oxidation of metal NPs include the work by Henglein ${ }^{79}$ and Plieth. ${ }^{80}$ Henglein described the particle size dependence of the standard redox potential of metal NPs. He showed that the standard oxidation/reduction potential $\left(\mathrm{E}^{\circ}\right)$ for bulk $\mathrm{Ag}$ shifts from $0.799 \mathrm{~V}$ to $-1.8 \mathrm{~V}$ for a single $\mathrm{Ag}$ atom. Later, Plieth developed theoretical equations to explain the shift in $\mathrm{E}^{0}$ of metal particles with a decrease in size from bulk size to smaller size. $\mathrm{He}$ predicted that the shift is proportional to the reciprocal of radius $(1 / r)$. The equation derived by Plieth showing the relationship between the shift in oxidation potential and particle radius is

$$
\Delta \mathrm{E}_{\mathrm{D}}=-2 \gamma \mathrm{V}_{\mathrm{M}} / \mathrm{zFr}
$$

Where $\Delta E_{D}$ is shift in oxidation potential between the bulk metal and NPs of a specific size, $\gamma$ is surface stress, $V_{M}$ is molar volume, $z$ is the number of electrons passed for oxidation of a single atom, $\mathrm{F}$ is Faraday's constant, and $\mathrm{r}$ is NP radius. Hence, the shift in redox potential of small metal NPs from their bulk form is proportional to the ratio of surface tension (or stress) to that of the radius $(\gamma / r)$. To explain in simple words, with a decrease in size the oxidation potential of metal NPs shifts to more negative values compared to their bulk analogues. 
Later, researchers attempted to correlate the theoretical results of size dependent oxidation with experimental results. For example, Brainina and coworkers ${ }^{81}$ discussed theoretical studies for oxidation of metal NPs and also performed experimental work in which they showed that metal NPs oxidize at a potential less positive than their bulk metal forms. Brainina and coworkers ${ }^{82}$ also observed experimentally that the substrate onto which the metal NPs are assembled can also affect their electrochemical behavior. They reported that the interaction between the metal NPs and the electrode onto which they are assembled affects their oxidation, which is observed in the form of a potential shift. A positive shift was observed in the case of strong interactions between metal NPs, while the electrooxidation of NPs occurred at a lower potential as compared to the bulk metal in the case of metal NPs assembled onto an inert electrode surface. Thus, the electrooxidation of metal NPs was found to be shifted negatively as compared to the bulk metal. Sieradzki and co-workers ${ }^{83}$ observed a size dependent oxidation of Pt NPs using scanning tunneling microscopy (STM), discovering that Pt NPs of 1-3 nm in size dissolve well below their the bulk dissolution potential. Buttry and coworkers ${ }^{84}$ observed a size-dependent shift in oxidation potential for anodic dissolution of water-soluble Pd NPs and observed their results to be in excellent agreement with the Plieth model, except for NPs less than $1 \mathrm{~nm}$ in size.

Our group used anodic stripping voltammetry (ASV) to study the size dependent oxidation of $\mathrm{Au}$ and Ag NPs. Ivanova and Zamborini ${ }^{85}$ studied the oxidation of different sizes of Ag NPs. They observed that the oxidation potential 
of $\mathrm{Ag}$ NPs increased to more positive values with an increase in the size of $\mathrm{Ag}$ NPs. The oxidation potential shifted from $275 \mathrm{mV}$ for $8 \mathrm{~nm} \mathrm{Ag} \mathrm{NPs} \mathrm{to} 318 \mathrm{mV}, 354$ $\mathrm{mV}$ and $382 \mathrm{mV}$ for $20 \mathrm{~nm}, 30 \mathrm{~nm}$ and $40 \mathrm{~nm}$ Ag NPs respectively, versus an $\mathrm{Ag} / \mathrm{AgCl}$ reference electrode. Ivanova and Zamborinis6 also studied the oxidation of different sizes of Au NPs in $\mathrm{KBr}$ electrolyte at potentials quoted versus $\mathrm{Ag} / \mathrm{AgCl}$ reference electrode showed that with a decrease in the size of the NP, the oxidation potential shifted to a negative value. Specifically, the average oxidation potential of Au NPs shifted from $913 \pm 19 \mathrm{mV}$ for $250 \mathrm{~nm}$ Au NPs to $734 \pm 1 \mathrm{mV}$ for $4.1 \mathrm{~nm}$ Au NPs. The correlation of their experimental results to the theoretical Plieth plot demonstrated excellent agreement between them. Masitas and Zamborini demonstrated that the oxidation potential of 1-2 nm diameter Au NPs shifted to $850 \mathrm{mV}$ negative as compared to the oxidation potential of bulk Au. Recently, Pattadar and Zamborini studied the oxidation potential of $1.6 \mathrm{~nm}$ Au NPs coated with a weak stabilizer, tetrakis(hydroxymethyl)phosphonium chloride (THPC) and observed that these NPs oxidized at $250 \mathrm{mV}$ negative from that of the citrate-coated $4.1 \mathrm{~nm}$ Au NPs. They also observed differences in the reactivity and stability of $1.6 \mathrm{~nm}$ and $4.1 \mathrm{~nm}$ Au NPs. The $1.6 \mathrm{~nm}$ Au NPs showed higher electrocatalytic activity towards $\mathrm{CO}_{2}$ reduction compared to $4.1 \mathrm{~nm}$ Au NPs however they were unstable to repetitive oxidation and reduction cycles in acidic solution while $4.1 \mathrm{~nm}$ Au NPs were stable under these conditions. This work besides showing the size dependent oxidation of metal NPs also showed the differences in stabilities and reactivities of metal NPs with a change in their size. In our work we aim to understand the role of SA/V on their oxidation behavior. 
$\mathrm{SA} / \mathrm{V}$ can be useful to understand the oxidation behavior of some unexplored metal nanostructures such as porous and aggregated NPs and to understand the role of assembly methods (which brings about changes in SA/V) on their peak oxidation potential $\left(E_{p}\right)$ in ASV.

\subsection{Importance of SA-to-Volume Ratio (SA/V) in the Analysis of Metal NPs}

Metal NPs have been widely used for different applications such as catalysis, sensing, nanoelectronics, imaging, therapeutics, and plasmonics. For the majority of these applications, the size of the metal NPs plays a key role. Smaller sized NPs usually show superior performance for catalytic and sensing applications due to their higher SA/V compared to bigger sized NPs or bulk materials. Considering this importance of $S A / V$, which is one of the relevant geometrical values associated with metal NPs, it is useful to measure the SA/V and correlate it to various fundamental properties of the metal NPs in order to use those properties for different applications. As mentioned already, various approaches such as under potential deposition, ligand adsorption method and BET adsorption method have been employed to measure the SA of metal NPs. Also, there are a few reports on the measurement of SA/V of bulk porous materials. However, there are hardly any methods available for measuring the SA/V for nanoparticulate systems, either porous or non-porous, especially for those confined to an electrode surface. SA/V is very important as it considers the volume

information of the NPs. This volume contribution in addition to their SAs is very important for relating their properties and assessing their usefulness for various 
applications such as catalysis, sensing and biomedical applications. It is possible that two NP-coated electrode surfaces with different-sized NPs could have the same SA due to a different loading of NPs on the electrode. They could have similar behavior, but without volume information, it would not be possible to determine which electrode was better in terms of activity per amount of material. Measuring the SA only would not provide the full information to assess the material. A less active material could appear more active simply because it had more volume of material on the electrode.

This dissertation describes the measurment of SA/V of NPs on an electrode surface by a very simple two-step approach using cyclic voltammetry (CV) followed by anodic stripping voltammetry (ASV). In the case of Au NPs, the reduction peak in a surface oxidation-reduction cycle in CV in acidic solution is directly related to the surface atoms and is thus indicative of the SA of the NPs. In ASV, the complete oxidative dissolution of all NPs using a suitable electrolyte (halide containing in case of Au NPs) is indicative of the total V of the NPs. This ratio of the reduction peak in CV and the oxidation peak in ASV gives the total electrochemical SA/V of the electrode-confined NPs. The development of this approach and application of it to characterize NP size, aggregation, assembly methods, and porosity are the main contributions of this dissertation. The following sections introduce these applications.

1.7.1. Electrochemical SA/V for Size Analysis of Metal NPs. Measuring the SA/V of a metal NP sample can be a good alternative to other methods for the 
analysis of spherical-shaped NPs. In the case of electrode-attached sphericalshaped NPs, the total SA $\left(n 4 \pi R^{2}\right)$ to volume $\left(n(4 / 3) \pi R^{3}\right)$ ratio of surface-attached NPs is given by

$S A / V=\left(n 4 \pi R^{2}\right) /\left(n(4 / 3) \pi R^{3}\right)=3 / R$

where is the total number of nanospheres on the electrode surface and $R$ is the radius of the metal nanosphere. Using this relation, the radius of the sphericalshaped NPs can be measured from which its diameter (size) can then be determined. This is shown schematically in Figure 1.5 and is the focus of Chapter III of this dissertation. When this ratio is correlated to a spherical geometry having the theoretical values of $3 / R$, the $R$ of the nanoparticle can be measured electrochemically. The size determination of metal NPs using this simple electrochemical SA/V is very useful due to its simple operation and cost effectiveness. The approach has been used to analyze the size of Au NSs successfully. ${ }^{87-88}$ 


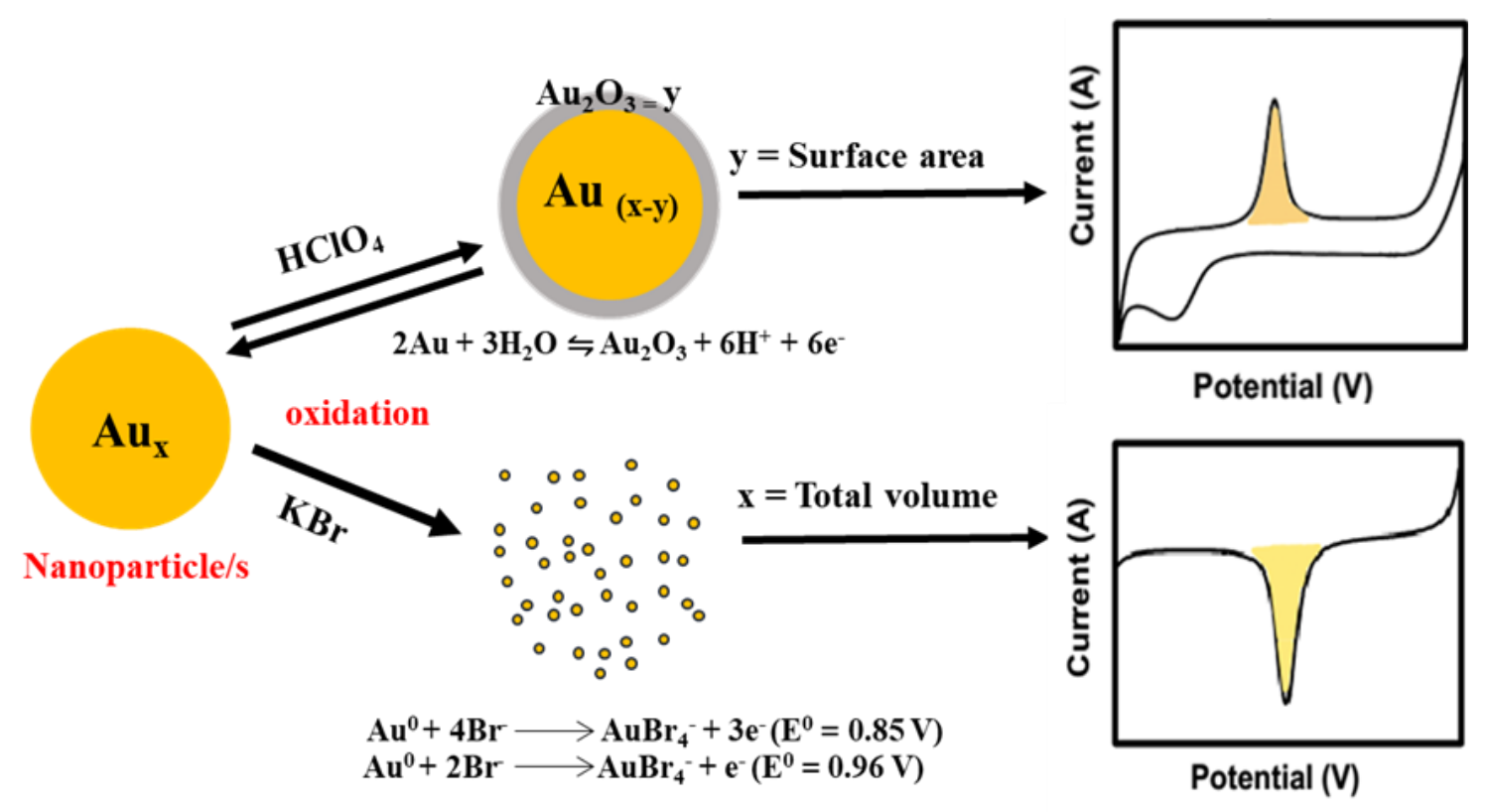

Figure 1. 5. Schematic representation of the principle involved in the determination of SA/V of Au NPs for determining their average size.

\subsubsection{Electrochemical SA/V for Aggregation Analysis of Metal NPs.}

The aggregation state of metal NPs plays an important role in various areas and, in fact, has shown advantages over their non-aggregated counterparts for various applications, such as certain catalytic reactions and in biological applications. For instance, Kim and coworkers ${ }^{89}$ found that partially aggregated Au NPs enhanced the reaction rates of 4-NP after irradiation with light as compared to monodisperse NPs, due to their higher photon-to-heat conversion efficiency caused by their broad absorption bands across the visible to near-IR region. Special aggregated states such as the dimeric and trimeric forms possess interstitial hot spots that 
enhance Raman scattering or fluorescence signals, improving the detection of analytes of interest through spectroscopy and sensing. ${ }^{90}$

Lakowicz_and coworkers ${ }^{91}$ found a 13-fold increase in the fluorescence signal for Cy5-labelled oligonucleotides sandwiched between a metal dimer compared to the monomer form. Kotov and coworkers ${ }^{92}$ developed controlled assemblies (aggregates) of NPs with DNA oligomers which can potentially act as cellular "drones" for detecting metabolic products that governs cellular activities. Lin and coworkers ${ }^{93}$ observed improved sensitivity to refractive index for trimers over their individual counterparts. Bonham and coworkers ${ }^{94}$ developed dimeric $\mathrm{Au}$ NPs using DNA duplexes which contained a recognition site for a specific DNAbinding protein and used them to study DNA-protein interactions. With such useful applications, it makes sense to characterize these aggregated states in NPs in order to better understand their fundamental properties such as stability, oxidation behavior and collision frequency with electrode surfaces. There are reports on the positive shift in the peak oxidation potential $\left(E_{p}\right)$ in the ASV of aggregated metal NPs as compared to well-isolated, non-aggregated metal NPs.

Electrochemical SA/V is another good alternative to $E_{p}$ measurements as it gives information about the SA contribution besides the $\mathrm{V}$ of these aggregate states of metal NPs and is helpful to better understand their fundamental oxidation properties. ${ }^{95}$ The SA/V is determined by a similar approach as that of nonaggregated metal NPs (Figure 1.6) as described in Chapter IV. 

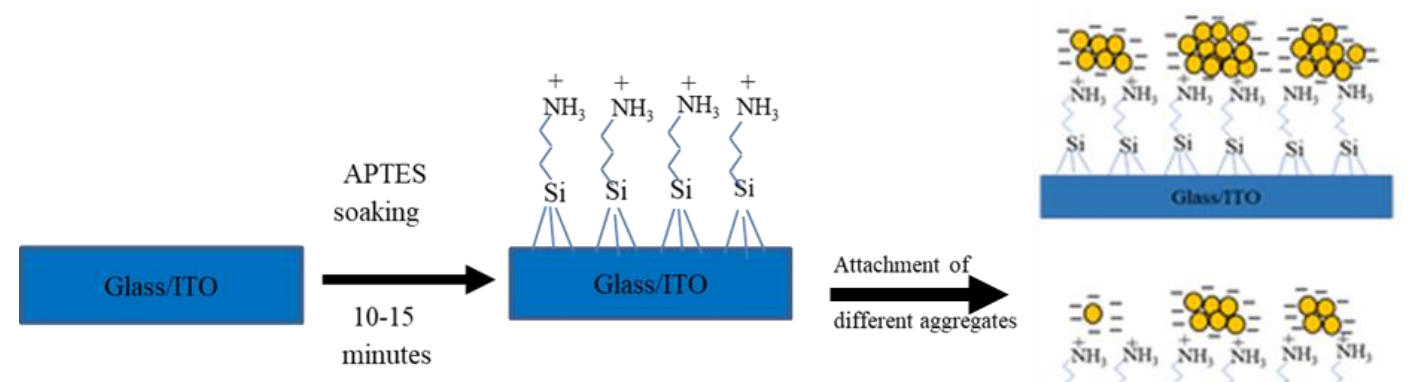

Si Si Si si si si

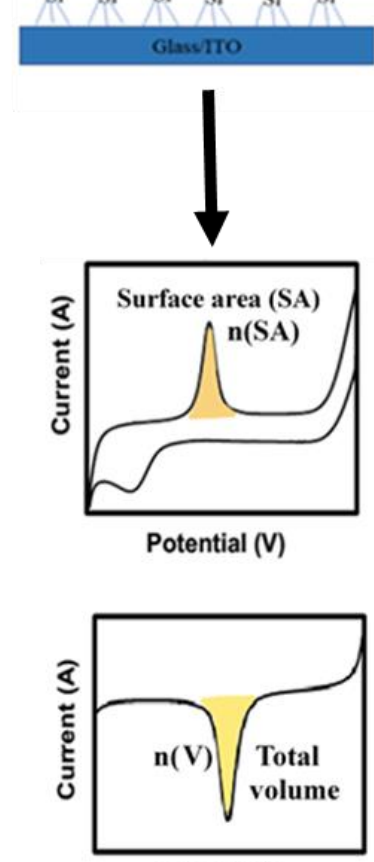

Potential (V)

Figure 1. 6. Schematic representation of the steps for the determination of SA/V of different aggregated states of Au NPs. The aggregates of Au NPs are formed after addition of acid (perchloric acid), which causes the neutralization of citrate group on the NPs. The aggregated NPs are then assembled on glass/ITO to measure SA/V. 


\subsubsection{Electrochemical SA/V Determination of Metal NPs Assembled by Different Assembly Methods on Glass/ITO Electrode.}

For their varied applications, metal NPs are assembled on the surface of different substrates or electrode materials by different assembly methods. The interactions of the metal NPs with different electrode materials or assembled by different assembly methods alter their behavior due to differences in surface energies which is related to their SA/V. ${ }^{96}$ This is evident from differences in their stabilities on different electrode materials ${ }^{97}$ and for different assembly methods. This is experimentally studied by observing differences in their $E_{p}$ in ASV, but it is not clear how or if that relates to their SA/V. However, if the SA contribution is considered along with the total V information for NPs assembled by different methods (Figure 1.7), it would provide valuable fundamental information about what controls NP oxidative stability and provide practical information needed to optimize the metal NP-supported electrode surface for maximum performance and stability for electrocatalysis and electrochemical sensing applications. Chapter V focuses on electrochemical $\mathrm{SA} / \mathrm{V}$ measurements and the correlation to their oxidative stability $\left(E_{p}\right)$ for metal NPs assembled as shown in Figure 1.7. 
A. NPs attachment to electrode through molecular linker

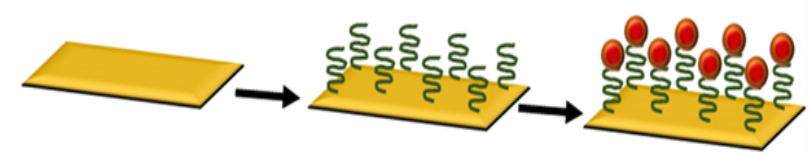

B. NPs assembled on electrode by dropcast deposition after carbon black mixing

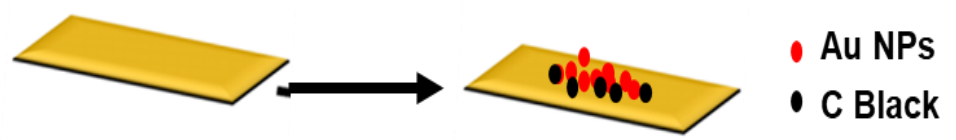

C. NPs attachment to electrode by drop casting method

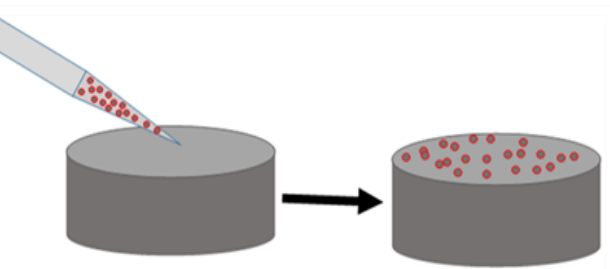

D., NPs attachment to electrode by electrophoretic deposition method

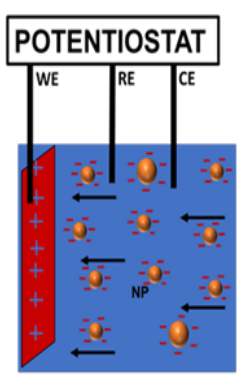

E. Direct NPs formation on electrode surface by electrochemical deposition

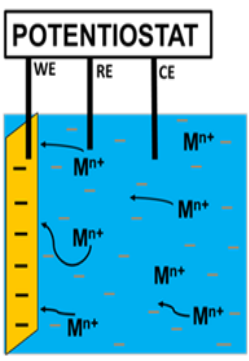

Figure 1. 7. Schematic representation of different assembly methods of NPs on electrode surfaces. 


\subsubsection{Electrochemical SA/V for Analysis of Porous Metal NPs.}

Porous nanostructures have recently gained special interest for various applications such as catalysis, biosensing, and energy storage due to their large reaction SA/V. Different approaches have been employed for synthesizing porous bulk or nanostructured metals. These include etching of bulk metal electrodes, ${ }^{98-}$ ${ }^{99}$ electrodeposition, ${ }^{100-101}$ and selective dealloying of a less noble metal in a metal NP alloy. ${ }^{102-103}$ Snyder and coworkers ${ }^{104}$ de-alloyed $\mathrm{AgAu}$ alloys in neutral $\mathrm{pH}$ $\mathrm{AgNO}_{3}$ solution to form porous nanostructures having $\mathrm{SA}$ three times greater than those formed by dealloying in nitric acid solution. Zhang and coworkers ${ }^{105}$ prepared Au-based nanoboxes and nanocages having controlled porosity by dissolving $\mathrm{Ag}$ from $\mathrm{Au}-\mathrm{Ag}$ alloy nanoboxes using $\mathrm{H}_{2} \mathrm{O}_{2}$. These dealloyed nanoboxes and nanocages had high SA and were also useful to detect $\mathrm{H}_{2} \mathrm{O}_{2}$. Kim and coworkers ${ }^{106}$ synthesized highly porous Au core/porous shell NPs (CPS NPs) by selectively dissolving $\mathrm{Ag}$ atoms from $\mathrm{Au} / \mathrm{Au}-\mathrm{Ag}$ core/alloy shell NPs. The CPS NPs showed very short induction time, low activation energy, high conversion rate constant and high turnover frequency due to their catalytically active porous shells, which contained surface defects, ultra-high porosity and photothermal properties. There are methods available, such as BET, for determining the SA of these porous nanostructures in order to correlate the measured SA/V with their function and stability in different applications. ${ }^{107-108}$ The mass (or volume) must be known by some other method, however, to determine the SA/V. It is critical to know the mass (or volume) since the SAV determines the surface energy and stability properties. SA is useless without knowing the V. Also, knowing SA/V is critical because it normalizes 
the active area to the total amount of material, which is very important to know for cost reasons. It is desirable to obtain the most SA for the least amount of total material to keep the cost low. Our electrochemical approach for measuring SA/V allows measuring both in a simple, fast, low cost manner, providing a useful measurement that is important for understanding the stability and applications of porous nanostructures. Figure 1.8 illustrates the preparation of porous NPs and the electrochemical measurement of their SA/V, which is the focus of Chapter VI of this dissertation. An advantage of this approach is that it occurs directly on the electrode surface and prior knowledge of the amount of material or density is not needed.

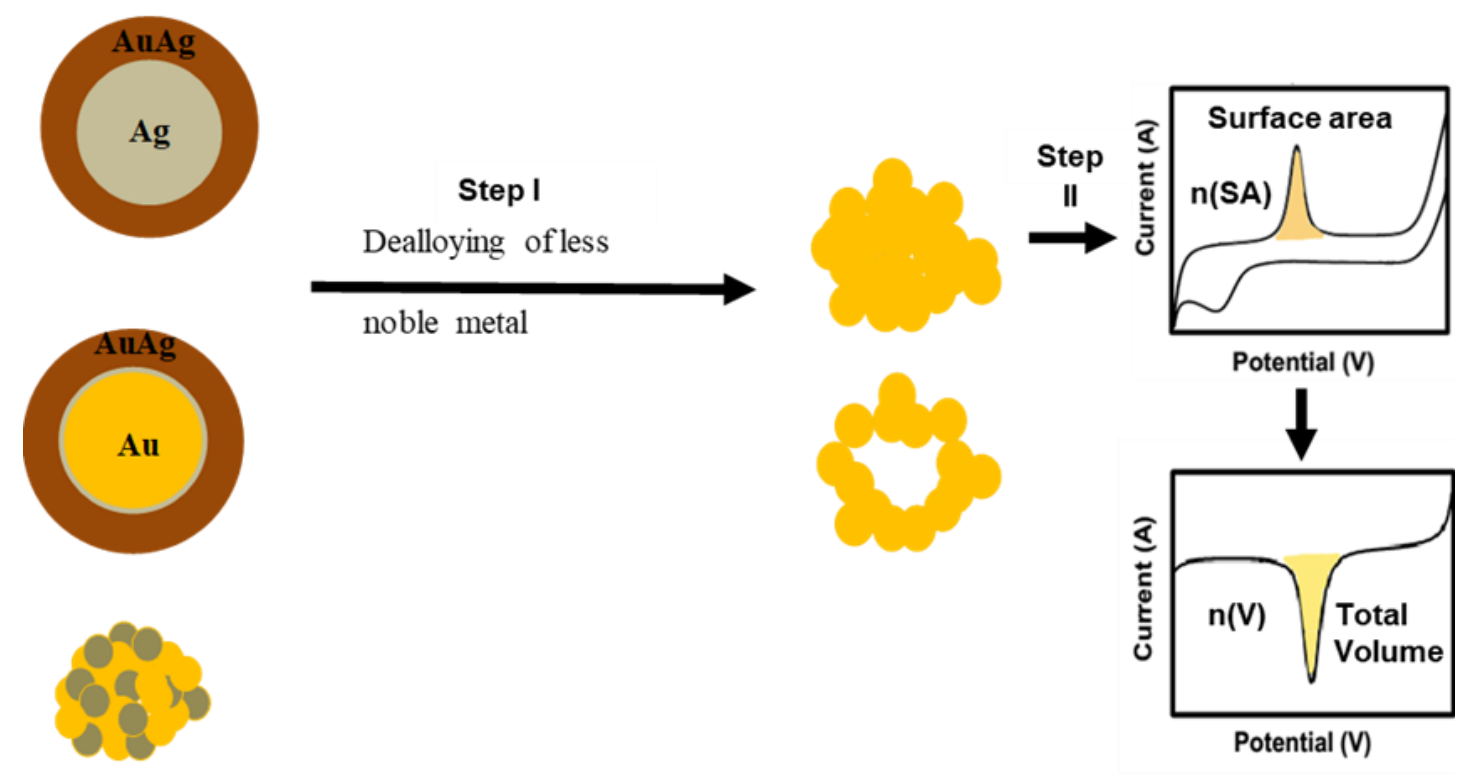

Figure 1. 8. Schematic representation of selective dealloying of core shell/mixed alloys of AuAg system for formation of porous $\mathrm{Au}$ nanostructures and determination of SA/V of the porous Au nanostructures. The AuAg alloys or core 
shell nanostructures are formed by chemical synthesis, assembled on electrode surfaces, and dealloyed to remove Ag from them (Step I). After this, the SA/V of the $\mathrm{Au}$ nanostructures remaining on the electrode surface is determined electrochemically (Step II). 
CHAPTER II

\section{EXPERIMENTAL METHODS}

This chapter describes the experimental procedures involved in this dissertation. Figure 2.1 shows the general scheme of experiments involved in this work. The steps include preparation of the electrode, synthesis of Au nanospheres (NSs), attachment of Au NSs to the electrode material, and characterization by photospectroscopy, microscopy and electrochemical techniques.

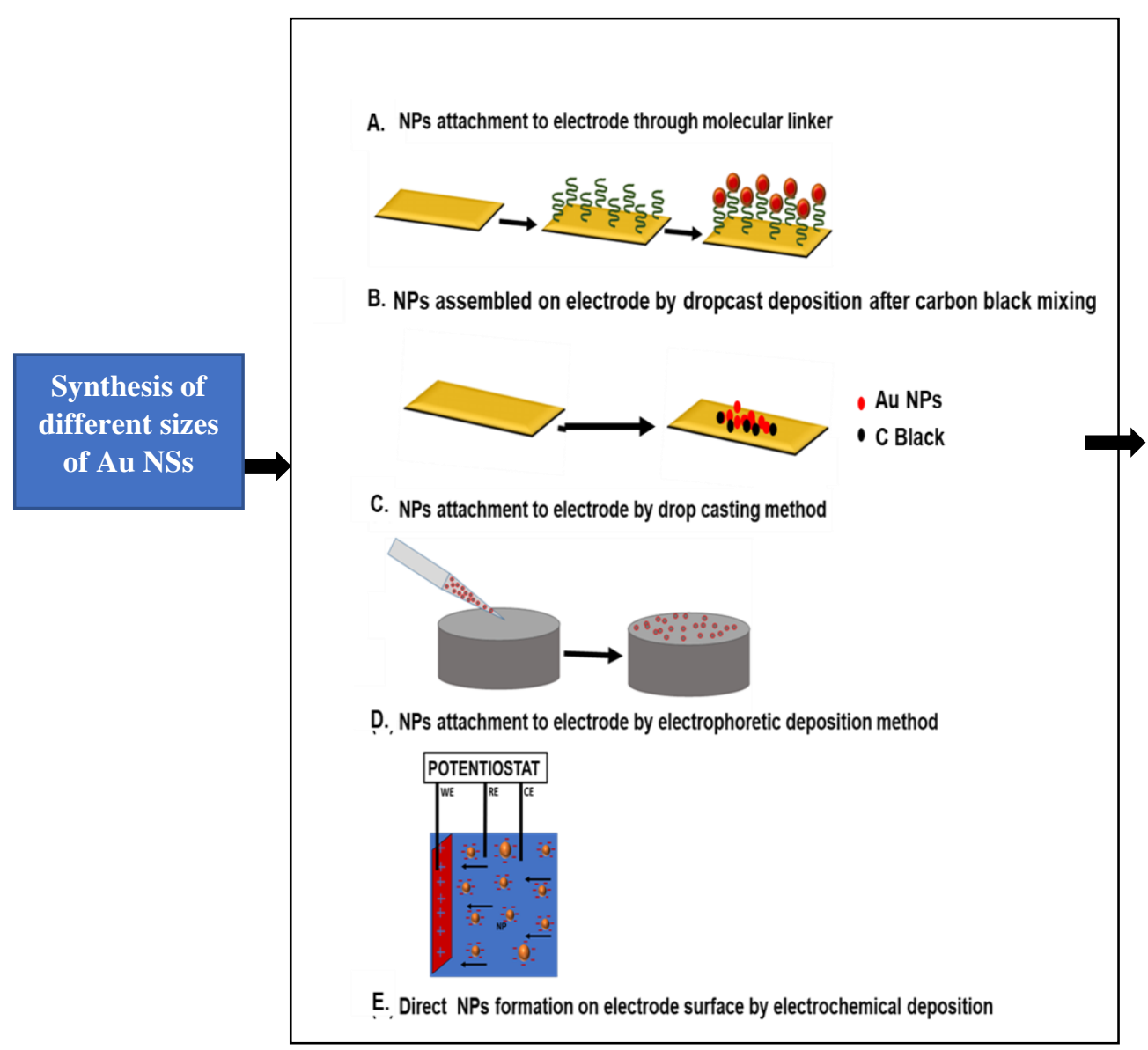

Characterization of Au NSs

\section{Electrochemical}

(Cyclic

Voltammetry,

Anodic Stripping

Voltammetry,

Chronocoulometry)

2. Microscopic

(Scanning Electron

Microscopy)

Attachment of Au NSs by different assembly methods 
Figure 2. 1. General scheme of the experimental procedures involved in this dissertation.

\subsection{Indium-Tin-Oxide-Coated Glass Electrodes (glass/ITO).}

Indium-tin-oxide (ITO or tin-doped indium oxide) is comprised of indium (III) oxide $\left(\ln _{2} \mathrm{O}_{3}\right)$, usually $90 \%$ by weight, and tin (IV) oxide $\left(\mathrm{SnO}_{2}\right)$, usually $10 \%$ by weight. Owing to its electrical conductivity and optical transparency, it is widely used for electrochemical and solar cell applications. In our case we used unpolished float (soda - lime) glass coated ITO slides (Delta Technologies, LTD) as an electrode to carry out the electrochemical experiments and to perform UV Vis measurements on Au NSs attached to it. These ITO coated slides have a surface roughness of $<0.2 \mathrm{IIm} / 20 \mathrm{~mm}$ and are coated either on one or both sides of the glass, with a resistance of 8-12 ohms. For using them as electrodes, the slides were cut using a diamond pen into $25 \times 7 \mathrm{~mm}$ slices followed by cleaning by sonicating in three solvents (acetone, ethanol, and 2-propanol) for 20 minutes in each. Finally, they are dried under a $\mathrm{N}_{2}$ stream before being used for the experiments.

\subsection{Chemicals and Reagents}

$\mathrm{HAuCl}_{4} \cdot 3 \mathrm{H}_{2} \mathrm{O}$ was synthesized from metallic $\mathrm{Au}(99.99 \%)$ in our lab. Sodium borohydride, hydrogen peroxide (30 wt.\%), (3-aminopropyl) triethoxysilane ( $\geq 98.0 \%$ ), 2-propanol (ACS reagent), Silver nitrate (ACS reagent, $\geq 99.0 \%$ ) and hydrogen peroxide solution (Sigma-Aldrich $30 \mathrm{wt} \%$ ) were purchased from Sigma Aldrich. Ethyl alcohol and acetone (ACS/USP grade) were purchased 
from Pharmco-AAPER. Trisodium citrate salt, potassium perchlorate (99.0$100.5 \%$ ), potassium bromide (GR ACS), and perchloric acid (60\%) were purchased from Bio-Rad laboratories, Beantown Chemical, EMD, and Merck, respectively. Tetrakis(hydroxymethyl)phosphonium Chloride $(80 \%$ solution in water) was purchased from ACROS ORGANICS. Sodium hydroxide (solid) was purchased from Fisher Scientific. Carbon Vulcan (>99\%) was purchased from Alfa Aesar and acetonitrile from Honeywell Burdick and Jackson (HPLC grade). NANO pure ultrapure water (Barnstead, resistivity of $18.2 \mathrm{M} \Omega-\mathrm{cm}$ ) was used to prepare all aqueous solutions.

\subsection{Aqua Regia Solution}

Aqua regia solution is a mixture of nitric acid $\left(\mathrm{HNO}_{3}\right)$ and hydrochloric acid $(\mathrm{HCl})$ in a 3:1 ratio, respectively. It is commonly used to clean glassware and clean off organic residues. The preparation of aqua regia and it's use to clean the glasswares is performed under the hood. The glassware was cleaned by aqua regia by filling them with the aqua regia and then rinsed with nanopure water several times before being used for the experiments.

\subsection{Au and AuAg Alloy Nanosphere Synthesis}

\subsubsection{Synthesis of $1.6 \mathrm{~nm}$ Average Diameter THPC-Stabilized Au} Nanospheres (NSs).

We synthesized $1.6 \mathrm{~nm}$ diameter THPC-stabilized Au NSs using the procedure described by Duff and co-workers. ${ }^{109}$ Briefly, $500 \mu \mathrm{L}$ of $0.2 \mathrm{M} \mathrm{NaOH}$ 
solution was added to glass vials containing $15.16 \mathrm{~mL}$ of nanopure water followed by the addition of $400 \mu \mathrm{L}$ of the reducing agent THPC $(200 \mu \mathrm{L}$ of $80 \%$ THPC diluted to $16.66 \mathrm{~mL}$ of nanopure water). Finally, $660 \mu \mathrm{L}$ of $25 \mathrm{mM} \mathrm{HAuCl} 4_{4} \cdot 3 \mathrm{H}_{2} \mathrm{O}$ was added to the vials with constant stirring. After the addition of $\mathrm{HAuCl}_{4} \cdot 3 \mathrm{H}_{2} \mathrm{O}$, immediately an orange-brown color formed in solution, indicative of small Au NPs.

\subsubsection{Synthesis of $4 \mathrm{~nm}$ Average Diameter Citrate-Stabilized Au NSs.}

We synthesized $4 \mathrm{~nm}$ average diameter Au NSs as described by Murphy and co-workers. ${ }^{110}$ The method involved the addition of $0.5 \mathrm{~mL}$ of $10 \mathrm{mM} \mathrm{HAuCl}_{4} \cdot 3 \mathrm{H}_{2} \mathrm{O}$ and $0.5 \mathrm{~mL}$ of $10 \mathrm{mM}$ trisodium citrate to $20 \mathrm{~mL}$ of water followed by the addition of $0.6 \mathrm{~mL}$ of ice-cold $10 \mathrm{mM} \mathrm{NaBH}_{4}$ at once with rapid stirring for 2 hours. After the addition of $\mathrm{NaBH}_{4}$, the solution turned red immediately indicating the formation of NSs. Figure 2.2 below illustrates the procedure involved in the synthesis of 4 nm diameter citrate-coated Au NPs. 


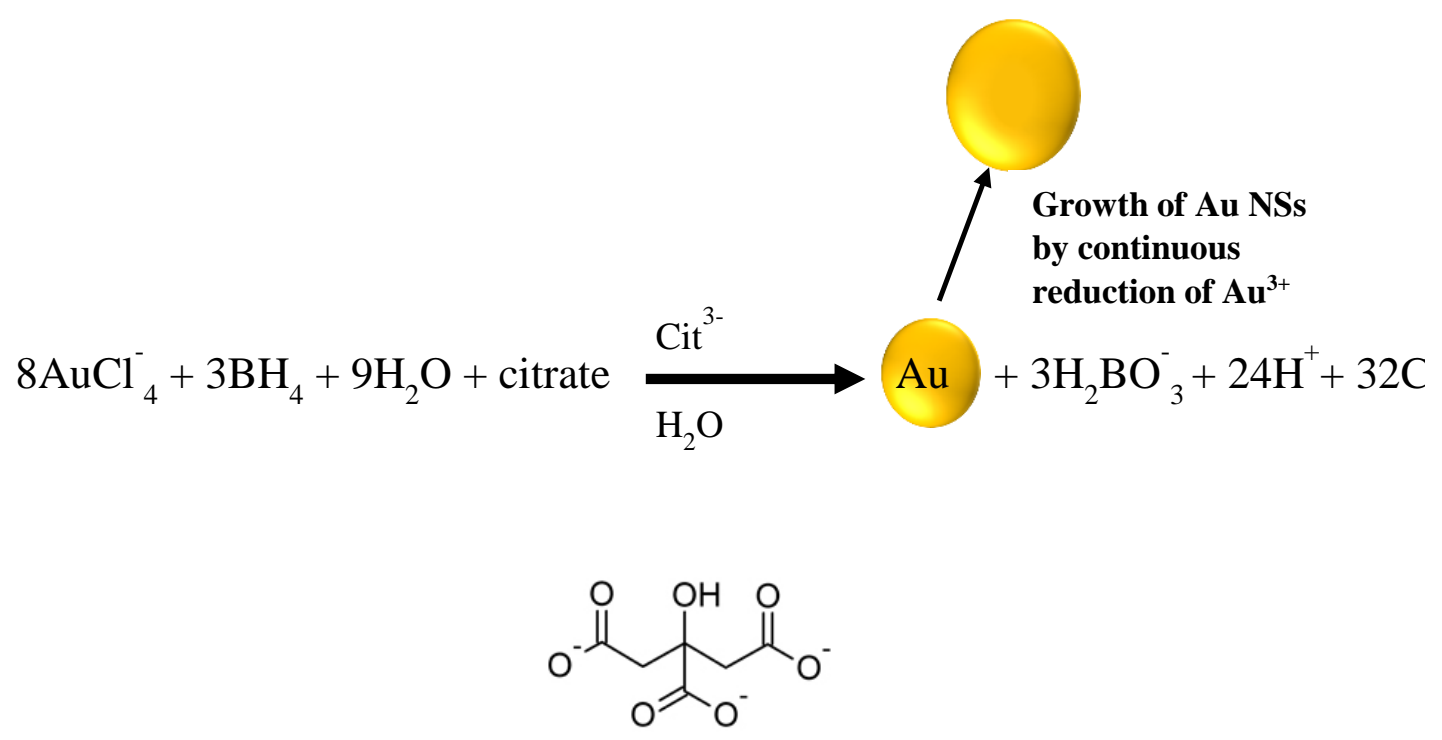

Structure of citrate (stabilizer)

Figure 2. 2. Procedure involved in the synthesis of $4 \mathrm{~nm}$ diameter citrate-coated Au NPs.

\subsubsection{Synthesis of $15 \mathrm{~nm}$ Average Diameter Citrate-Stabilized Au NSs.}

$15 \mathrm{~nm}$ average diameter Au NSs were synthesized by a modified Turkevich method. ${ }^{111}$ Briefly, $500 \mu \mathrm{L}$ of $0.01 \mathrm{M} \mathrm{HAuCl}_{4} \cdot 3 \mathrm{H}_{2} \mathrm{O}$ was added to $17.0 \mathrm{~mL}$ of nanopure water and heated to boiling. Immediately after boiling, $2.5 \mathrm{~mL}$ of $0.01 \mathrm{M}$ citric acid, trisodium salt solution was added and the solution was stirred for 10 minutes. Finally, the resulting solution was stirred for another 15 min at room temperature and allowed to cool. At this point, the solution turned to a bright red color indicating the formation of Au NSs. Figure 2.3 below illustrates the synthesis of $15 \mathrm{~nm}$ Au NSs by the citrate reduction method. 


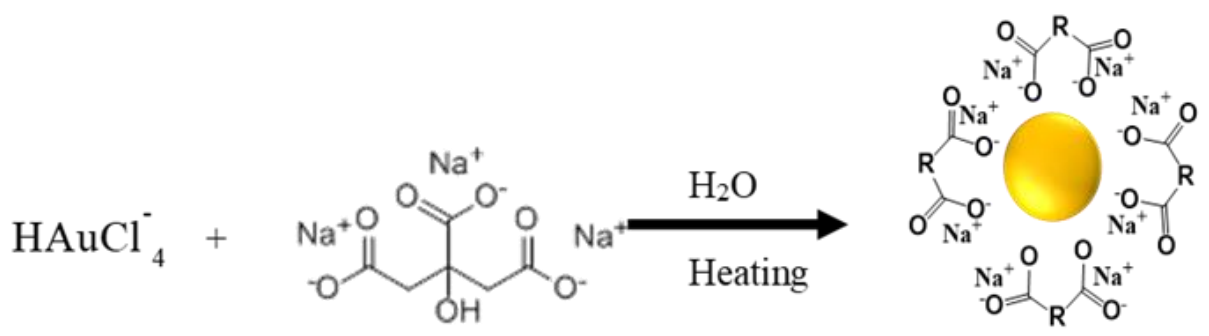

Trisodium citrate

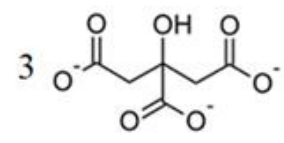

citrate

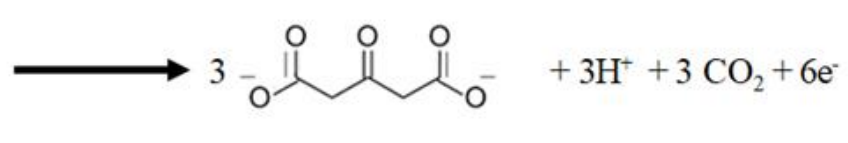

acetonedicarboxylate

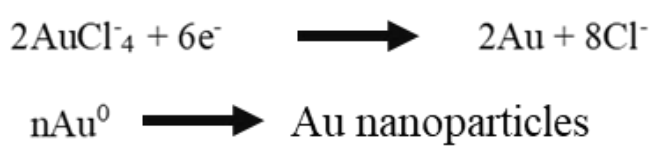

Figure 2. 3. Procedure for the synthesis of $15 \mathrm{~nm}$ Au NSs by the citrate reduction method.

\subsubsection{Synthesis of 32,50 and $70 \mathrm{~nm}$ Average Diameter Citrate-Stabilized Au} NSs.

We used the seed-mediated growth method described by Wang and coworkers $^{112}$ to prepare fairly monodisperse citrate-stabilized Au NSs that were 32,50 , and $70 \mathrm{~nm}$ in average diameter. For $32 \mathrm{~nm}$ diameter NSs, $5 \mathrm{~mL}$ of 30 weight $\% \mathrm{H}_{2} \mathrm{O}_{2}$ was added to an aqueous solution containing $4.90 \mathrm{~mL}$ of nanopure water, $20 \mu \mathrm{L}$ of $25 \mathrm{mM} \mathrm{HAuCl}_{4} \cdot 3 \mathrm{H}_{2} \mathrm{O}$ and $25 \mu \mathrm{L}$ of $38.8 \mathrm{mM}$ trisodium citrate under stirring immediately followed by injection of $100 \mu \mathrm{L}$ of $15 \mathrm{~nm}$ diameter Au NSs with 
continued strirring. After the addition of $15 \mathrm{~nm}$ diamter Au seed NSs, the color of the solution changed within 1 min to a pink red color, suggesting the formation of larger Au NSs. We synthesized $50 \mathrm{~nm}$ diameter Au NSs by the same procedure, but by adding $33 \mu \mathrm{L}$ of $25 \mathrm{mM} \mathrm{HAuCl}_{4} \cdot 3 \mathrm{H}_{2} \mathrm{O}$ and $50 \mu \mathrm{L}$ of $15 \mathrm{~nm}$ diameter $\mathrm{Au}$ NSs. We synthesized $70 \mathrm{~nm}$ diameter Au NSs by adding $50 \mu \mathrm{L}$ of $25 \mathrm{mM} \mathrm{HAuCl}_{4}$ $3 \mathrm{H}_{2} \mathrm{O}$ and $25 \mu \mathrm{L}$ of $15 \mathrm{~nm}$ diameter Au NSs. Figure 2.4 below illustrates the procedure for the seed-mediated growth of citrate-coated Au NPs by $\mathrm{H}_{2} \mathrm{O}_{2}$ reduction. Figure 2.4 below illustrates the scheme for seed-mediated growth of citrate coated $\mathrm{Au}$ NPs by $\mathrm{H}_{2} \mathrm{O}_{2}$ reduction method

$$
\begin{gathered}
\text { Au }+\mathrm{AuCl}_{4}^{-}+3 / 2 \mathrm{H}_{2} \mathrm{O}_{2}+\text { citrate } \longrightarrow \begin{array}{l}
\text { Au Growth } \\
\mathrm{Au} \\
\text { seed }
\end{array} \\
\mathrm{AuCl}_{4}^{-}+3 / 2 \mathrm{H}_{2} \mathrm{O}_{2} \longrightarrow 4 \mathrm{Cl}^{-}+3 \mathrm{H}^{+}+3 / 2 \mathrm{O}_{2}
\end{gathered}
$$

Figure 2. 4. Procedure for the seed-mediated growth of citrate-coated Au NPs by $\mathrm{H}_{2} \mathrm{O}_{2}$ reduction.

\subsection{Electrode Functionalization and Nanoparticle Attachment to Glass/ITO}




\subsubsection{Functionalization of glass/ITO electrodes with 3- aminopropyltriethoxysilane (APTES).}

Glass/ITO electrodes were cleaned by sonication in acetone, ethanol, and 2-propanol for $30 \mathrm{~min}$ in each solvent before drying under $\mathrm{N}_{2}$. The glass/ITO electrodes were then functionalized with APTES by immersing into a solution containing $100 \mu \mathrm{L}$ of APTES, $10 \mathrm{~mL}$ of 2-propanol, and 2 to 3 drops of nanopore water and heating at $85-90^{\circ} \mathrm{C}$ for $30 \mathrm{~min}$. After $30 \mathrm{~min}$, the electrode was thoroughly rinsed with 2-propanol and dried under $\mathrm{N}_{2}$. Figure 2.5a (left electrode) illustrates the functionalization of a glass/ITO electrode with APTES.

\subsubsection{Attachment of Au NSs to glass/ITO functionalized with APTES}

linker. The glass/ITO electrodes functionalized with APTES were soaked in a solution of NPs for a specific duration of time until a desired coverage of NPs were attached to the electrodes. Figure 2.5a below illustrates the attachment of Au NSs to glass/ITO through APTES.

\subsubsection{Attachment of Au NSs to glass/ITO by drop cast deposition. Au} NSs were drop cast deposited onto glass/ITO by dropping $25 \square \mathrm{L}$ of a Au NS solution onto the electrode surface and allowing it to air dry. Figure $2.5 \mathrm{~b}$ below shows the attachment of Au NSs through drop cast deposition onto glass/ITO. 

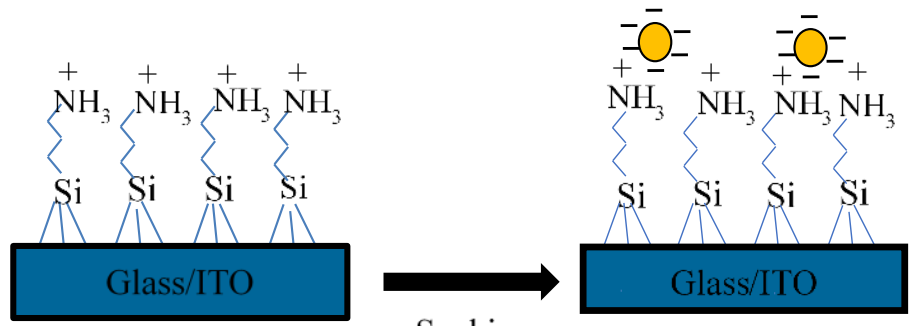

Soaking in $\mathrm{Au} \mathrm{NSs}$ solution

(a)
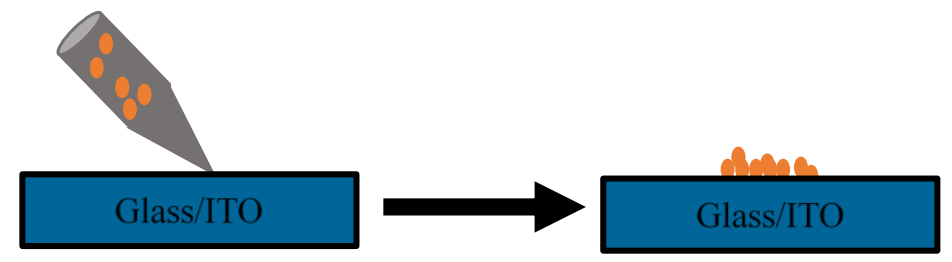

Drop casting of $\mathrm{Au}$ NSs on Glass/ITO

(b)

Figure 2. 5. Attachment of Au NSs to glass/ITO through (a) APTES and (b) drop cast deposition.

\subsubsection{Electrophoretic deposition (EPD) of Au NPs directly on} glass/ITO. Glass/ITO electrodes were cleaned by sonication for 20 min each in acetone, ethanol, and 2-propanol before drying under N2. Au NPs were then electrophoretically deposited onto the clean glass/ITO working electrode using a $\mathrm{CH}$ Instruments (Austin, TX) 660A electrochemical workstation in chronocoulometry mode with a gold foil connected with both counter and reference electrode. The composition of the EPD solution consisted of $5 \mathrm{~mL}$ of Au NPs +5 
$\mathrm{mL}$ of $0.1 \mathrm{M} \mathrm{HQ}+10 \mathrm{~mL}$ of $\mathrm{H}_{2} \mathrm{O}$. A fixed positive potential of $0.8 \mathrm{~V}, 1.2 \mathrm{~V}, 2.0 \mathrm{~V}$, $2.2 \mathrm{~V}$ and $2.5 \mathrm{~V}$ corresponding to $4 \mathrm{~nm}, 15 \mathrm{~nm}, 32 \mathrm{~nm}, 50 \mathrm{~nm}$ and $70 \mathrm{~nm}$ was applied to the glass/ITO immersed in this solution relative to the Au foil which drives the negatively-charged $\mathrm{Au}$ NPs to the positive glass/ITO electrode by electrophoresis. The deposition mechanism involves catalytic oxidation of hydroquinone $(\mathrm{HQ})$ which releases protons and neutralizes the negatively-charged citrate groups on the Au NSs, leading to their deposition onto the glass/ITO electrode. Figure 2.6 below illustrates the EPD set-up of Au NSs on glass/ITO. The $1.6 \mathrm{~nm}$ Au NSs were attached to the glass/ITO electrode by directly soaking the glass/ITO in the NPs solution since EPD was not possible on those positivelycharged NSs. 


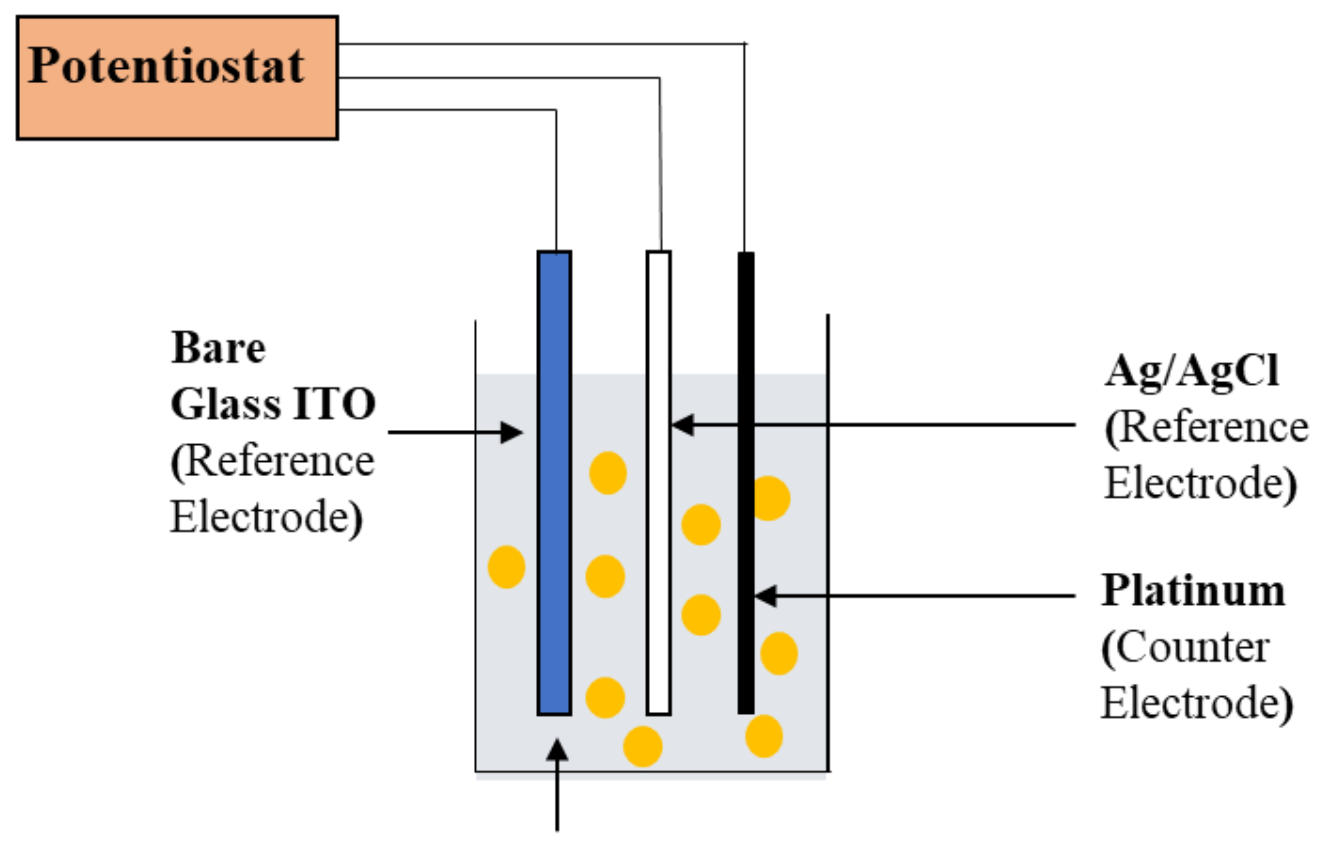

Au NSs solution + HQ (Hydroquinone)

Figure 2. 6. Scheme showing the set-up for the electrophoretic deposition (EPD) of $\mathrm{Au}$ NSs on glass/ITO.

\subsection{Characterization and Instrumentation}

2.6.1. Electrochemical Methods. This section describes the setup of the electrochemical cell and the different electrochemical techniques used for this work. The general set up showing the basic components of the electrochemical cell is as shown in Figure 2.7 below. The basic components include three electrodes immersed in a beaker containing electrolyte connected to a potentiostat and a computer used for collection and analysis of the data. The three electrodes include working, reference and counter electrodes and are as follows: 
1) Working electrode - This is the electrode where the electrochemical reaction of interest takes place.

2) Reference electrode - This is an electrode with a known potential, and it acts as a reference to measure and control the potential of the working electrode. Current does not run through it, allowing it to maintain the potential.

3) Counter electrode - This electrode is used to close the circuit. It is used to pass the current required to balance the current at the working electrode.

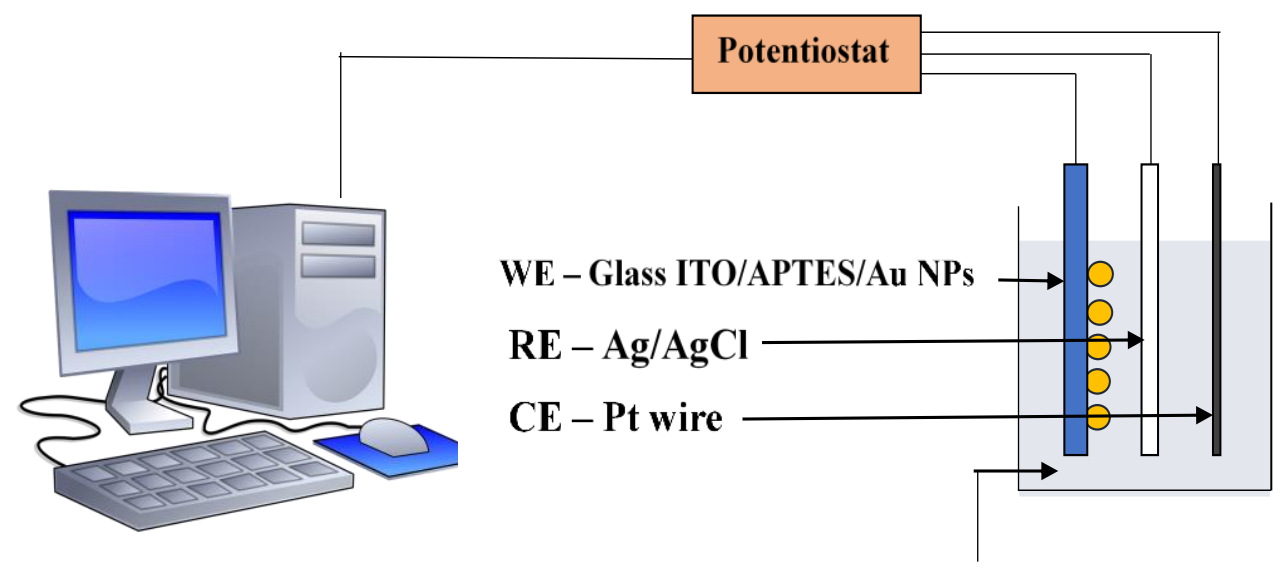

$0.1 \mathrm{M} \mathrm{HClO}_{4}$ for Cyclic Voltammetry $0.01 \mathrm{M} \mathrm{KBr}$ (or $0.01 \mathrm{M} \mathrm{KCl}$ ) in $0.1 \mathrm{M} \mathrm{HClO}_{4}$ for Anodic Stripping Voltammetry

Figure 2. 7. Illustration of the electrochemical set up.

In our work, we used metal NSs assembled by different assembly methods to the glass/ITO as the working electrode, $\mathrm{Ag} / \mathrm{AgCl}(3 \mathrm{M} \mathrm{KCl})$ as the reference electrode and a Pt wire as the counter electrode. We varied the electrolyte based on the requirements of our experiments. It is specified in the corresponding experiment. 
The potentiostat is an electronic instrument used to perform the electroanalytical experiments. The potentiostat is used to control the potential between the working and reference electrodes and also to measure the current flowing through the working and counter electrodes as a function of potential or as a function of time at a constant potential. For our work, we used a $\mathrm{CH}$ Instrument (Austin, TX) 630C electrochemical workstation for performing electrochemical CV and ASV.

\subsubsection{Anodic Stripping Voltammetry (ASV). ASV involves} measurement of the current at the working electrode as the potential between the working and the reference electrode is swept linearly with time. It thus measures the current as a function of potential. For our work we scanned the potential from a lower limit to upper limit or vice versa. The analyte undergoing oxidation or reduction at a specific applied potential was measured as current at that potential, leading to a peak in the plot. The ASV peak gives information about the thermodynamics of the reaction, rate of electron transfer, and the species undergoing oxidation/reduction. ASV also provides the concentration of the electroactive species. Integration of the peak produced by the current from an oxidation or reduction of the electroactive species provides the total charge $(\mathrm{Q})$ in Coulombs that was passed during the reaction. The amount of charge transferred at the working electrode is proportional to the concentration of the electroactive species oxidized/reduced at the surface of an electrode and this follows Faraday's $1^{\text {st }}$ law. This law is as follows:

$$
\mathrm{mol}=\mathrm{Q} / \mathrm{nF}
$$


where mol denotes number of moles of electroactive species being oxidized or reduced, $Q$ is the amount of electric charge in Coulombs passed through the working electrode during the oxidation or reduction reaction, $\mathrm{F}$ is Faraday's constant $(96,500 \mathrm{C} / \mathrm{mole})$, and $\mathrm{n}$ is the number of electrons transferred per molecule.

In our work we used ASV to analyze the size, aggregation, effect of assembly methods and electrode materials and porosity of metal NPs with the case of Au metal NSs. Figure 2.8 represents a typical ASV of $15 \mathrm{~nm}$ Au NSs attached to a glass/ITO electrode through an APTES linker. The ASV was performed at a scan rate of $1 \mathrm{mV} / \mathrm{s}$ in a mixture of $0.01 \mathrm{M} \mathrm{KBr}$ plus $0.1 \mathrm{M} \mathrm{KClO}_{4}$ solution. The potential was scanned from an initial potential of $-0.2 \mathrm{~V}$ to a final potential of $1.2 \mathrm{~V}$. A peak at $760 \mathrm{mV}$ indicates oxidation of $\mathrm{Au}$ in this $\mathrm{Br}^{-}$containing solution to the soluble $\mathrm{AuBr}_{2}^{-}$and $\mathrm{AuBr}_{4}^{-}$complex ions. The oxidation occurs by the following two reactions.

$$
\begin{aligned}
& \mathrm{Au}^{0}+4 \mathrm{Br} \longrightarrow \mathrm{AuBr}^{-}+3 \mathrm{e}^{-}\left(\mathrm{E}^{0}=0.85 \mathrm{~V} \text { vs. NHE }\right) \\
& \mathrm{Au}^{0}+2 \mathrm{Br} \longrightarrow \mathrm{AuBr}_{2}^{-}+\mathrm{e}^{-}\left(\mathrm{E}^{0}=0.96 \mathrm{~V} \text { vs. } \mathrm{NHE}\right)
\end{aligned}
$$

As discussed above the integration of the peak for oxidation of Au gives information about the concentration of Au NSs on the surface of the glass/ITO. The peak potential for oxidation $\left(E_{p}\right)$ is related to the thermodynamics of the oxidation process $\left(\mathrm{E}^{0}\right)$. ASV can be used to analyze important properties of metal NSs. ${ }^{113-}$ 115 


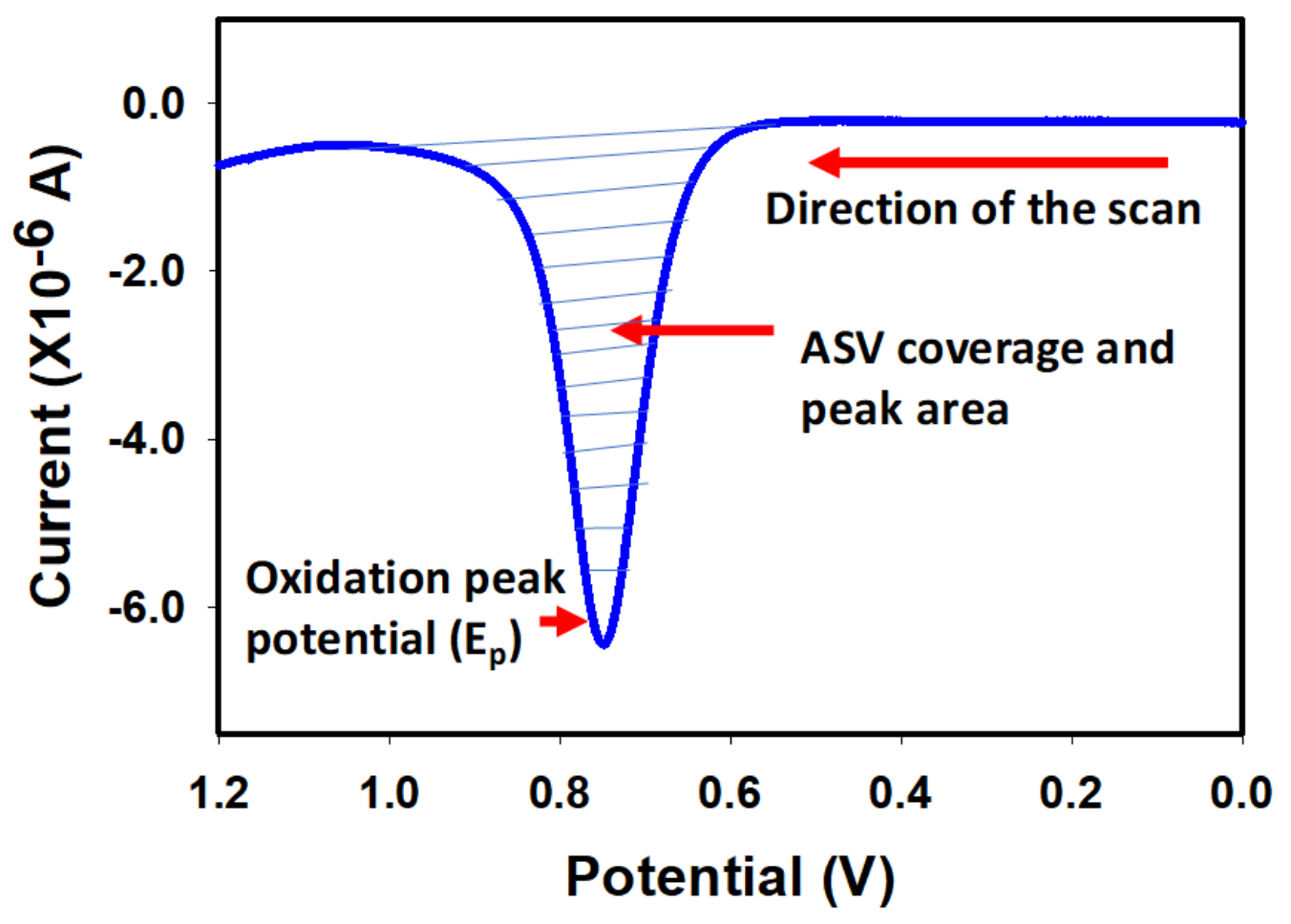

Figure 2. 8. Important parts of the anodic stripping voltammogram of Au NSs attached to glass/ITO.

\subsubsection{Cyclic voltammetry (CV). $\quad C V$ is another important} electrochemical method that is used to analyze electroactive species. The method measures the oxidation and reduction of the electroactive species. In CV a specific potential applied at the working electrode is swept at a certain rate and the current is measured as a function of potential. The electroactive species is subjected to potential scans at which they do not undergo any electrochemical reactions to a potential where they undergo redox reactions. ${ }^{116}$ Figure 2.9 shows a typical CV of $15 \mathrm{~nm}$ Au NSs attached to a glass/ITO electrode obtained in a $0.1 \mathrm{M} \mathrm{HClO}_{4}$ 


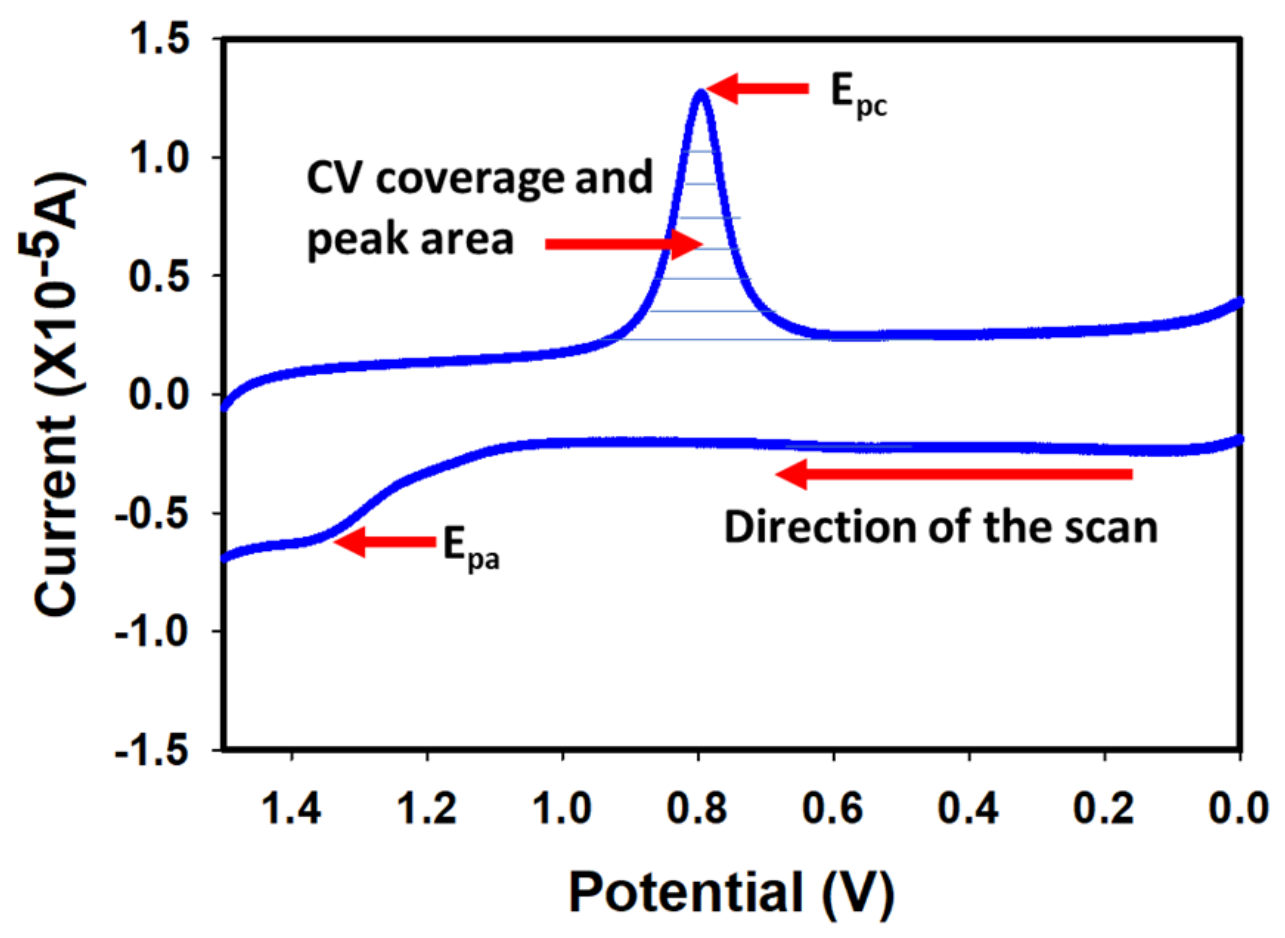

Figure 2. 9. Cyclic Voltammogram in $0.1 \mathrm{M} \mathrm{HClO}_{4}$ of $15 \mathrm{~nm}$ Au NSs attached to glass/ITO.

electrolyte solution. As labeled on the plot, $E_{p, a}$ is the potential for maximum anodic current obtained on the forward scan from $0.0 \mathrm{~V}$ to $1.5 \mathrm{~V}$, corresponding to the oxidative formation of a thin $\mathrm{Au}_{2} \mathrm{O}_{3}$ layer on the $\mathrm{Au}$ NS surface. $\mathrm{E}_{p, c}$ is the potential of maximum cathodic current formed on the reverse scan from $1.5 \mathrm{~V}$ back to 0.0 $\mathrm{V}$, corresponding to the reduction of the $\mathrm{Au}_{2} \mathrm{O}_{3}$ formed during the forward scan. The reversible oxidation-reduction process occurs in CV by the following reaction.

$$
\mathrm{Au}_{2} \mathrm{O}_{3}+6 \mathrm{H}^{+}+6 \mathrm{e}^{-} \leftrightharpoons 2 \mathrm{Au}+3 \mathrm{H}_{2} \mathrm{O}
$$

We used this type of analysis in the calculation of the SA of the Au NSs. 
2.6.1.3. Chronocoulometry (CC). In a chronocoulometric electrochemical experiment, a specific potential step is applied to a working electrode and the total charge $(Q)$ versus time is measured for electrochemical processes occurring at the working electrode. The $Q$ is determined by integrating current with time for an applied potential step. In this electrochemical technique a potential step is applied from a starting potential where there is no electrolysis to a point where oxidation or reduction of the electroactive species occurs in the electrolyte. In our work, we used CC for EPD of Au NPs of different sizes on the glass/ITO surfaces. We stepped to a specific potential depending upon the size of the Au NSs to be deposited. Larger sized NSs required higher potential. Figure 2.10 shows a typical CC plot obtained during the EPD of Au NSs.

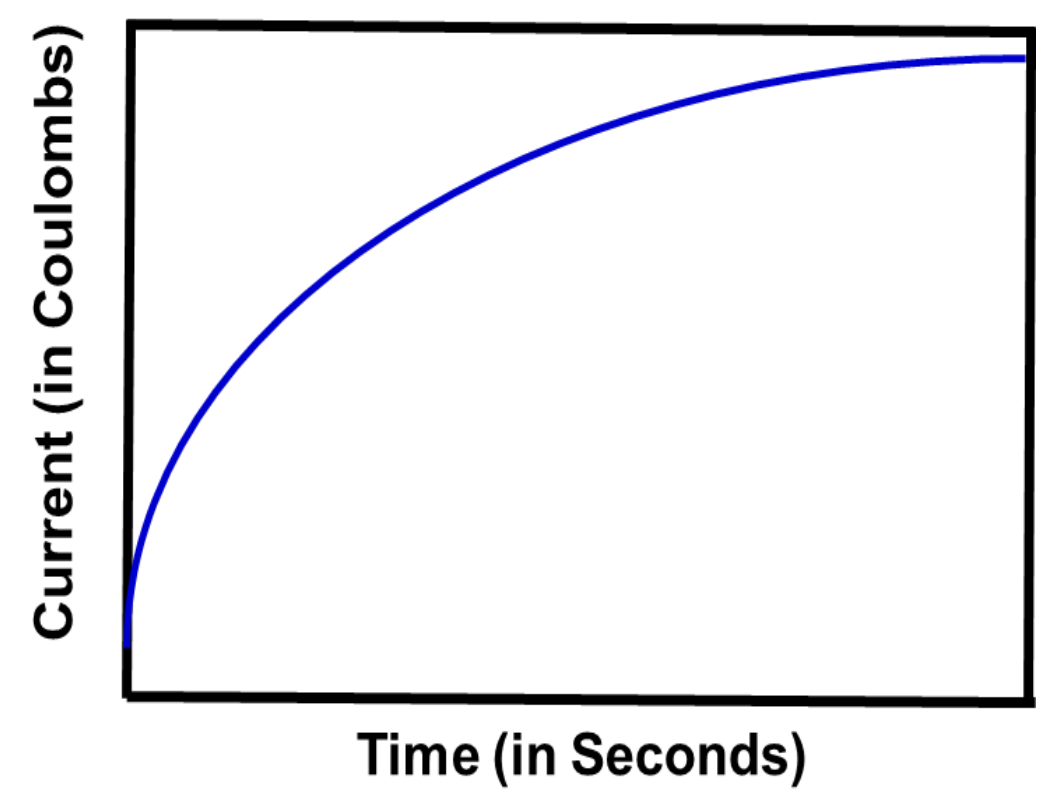

Figure 2. 10. Typical chronocoulometric curve for electrodeposition of Au NSs 


\subsubsection{Ultraviolet-Visible Spectroscopy (UV-vis). UV-vis spectroscopy}

relates the transmittance $T$ of a sample to its absorbance by Beer's-lambart law. For our work we used a Varian Cary 50 Bio UV-visible Spectrophotometer for measurement of UV-vis spectra. We obtained UV-vis spectra of Au NPs in solution as well as those attached to glass/ITO. For the solution phase samples, we placed the NP solution in a quartz or plastic cuvette, while we used the glass/ITO coated with Au NPs for surface-attached NPs. The samples were scanned from $900 \mathrm{~nm}$ to $300 \mathrm{~nm}$ and the absorbance measured as a function of wavelength. The transmittance of the samples of Au NPs in solution and the one's attached to surfaces were measured as the intensity of light that passes through the sample and denoted as I. The intensity of light that passes through the blank medium, water in the case of NPs in solution and glass/ITO without NPs in the case of electrode-attached NPs, is denoted as $\mathrm{I}_{0}$. The ratio of $\mathrm{I} / \mathrm{I}_{0}$ is called transmittance, T. The absorbance is calculated using a relation $A=e b c$. Figure 2.11 shows a UVvis spectrum of $15 \mathrm{~nm}$ Au NSs in aqueous solution.

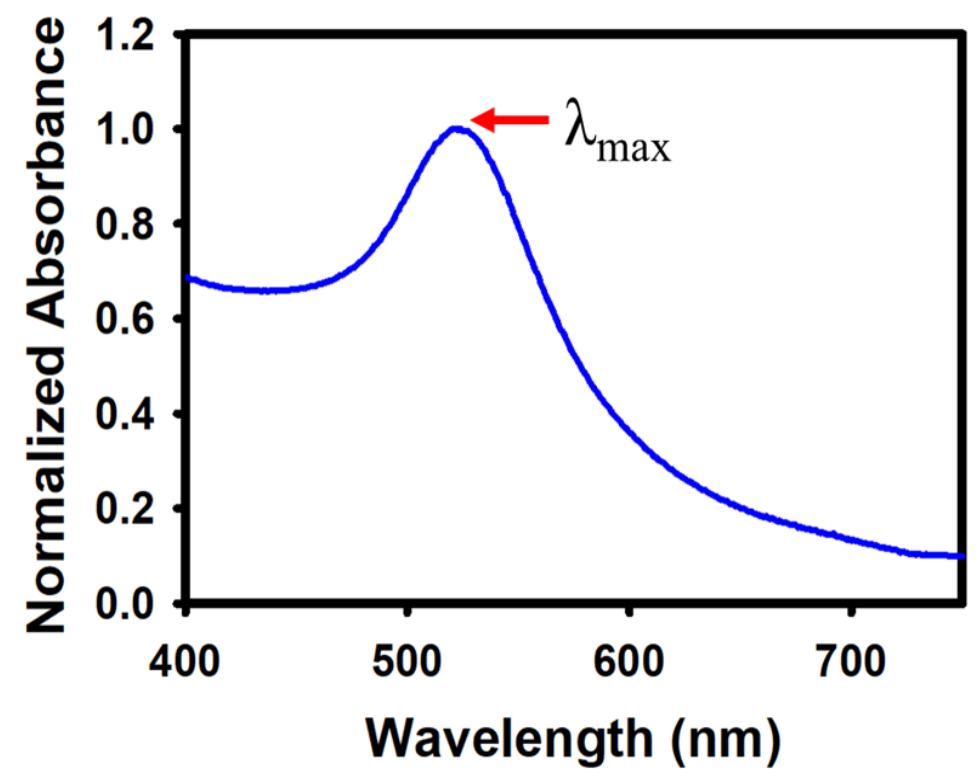


Figure 2. 11. UV-vis spectroscopy of $15 \mathrm{~nm}$ Au NSs

2.6.3. Scanning Electron Microscopy (SEM). Scanning electron microscopy is a commonly used and highly powerful tool for analyzing nanomaterials. In this a focused beam of electrons is used to scan the surface of a sample. These electrons interact with atoms in the sample which produces various signals that contain information about surface topography of the sample. SEM provides useful information on shape, size and aggregation of NPs. The typical components of SEM include an electron source (gun), electromagnetic lenses, apertures, a highvacuum environment, a specimen stage, electron beam scanning coils, signal detection and a processing system that provides real-time observation and image recording. For our work we imaged the Au NSs attached to glass/ITO using a Carl Zeiss STM AG Supra 35VP field emission scanning electron microscope (FESEM) operating at a voltage of $15 \mathrm{kV}$ and using an in-lens ion annular secondary electron detector. In SEM a beam of electrons hits the specimen surface which emits Xrays and three kinds of electrons, namely primary backscattered electrons, secondary electrons and Auger electrons. SEM mainly uses backscattered electrons and secondary electrons which are then picked up by an electron recorder and an imprint is recorded. This information is translated onto a detector screen that allows three-dimensional images of the sample surface to be represented clearly. Figure 2.12 illustrates the working principle of SEM (top) and shows a representative SEM image of $50 \mathrm{~nm}$ diameter Au NSs attached to a glass/ITO electrode (bottom). 


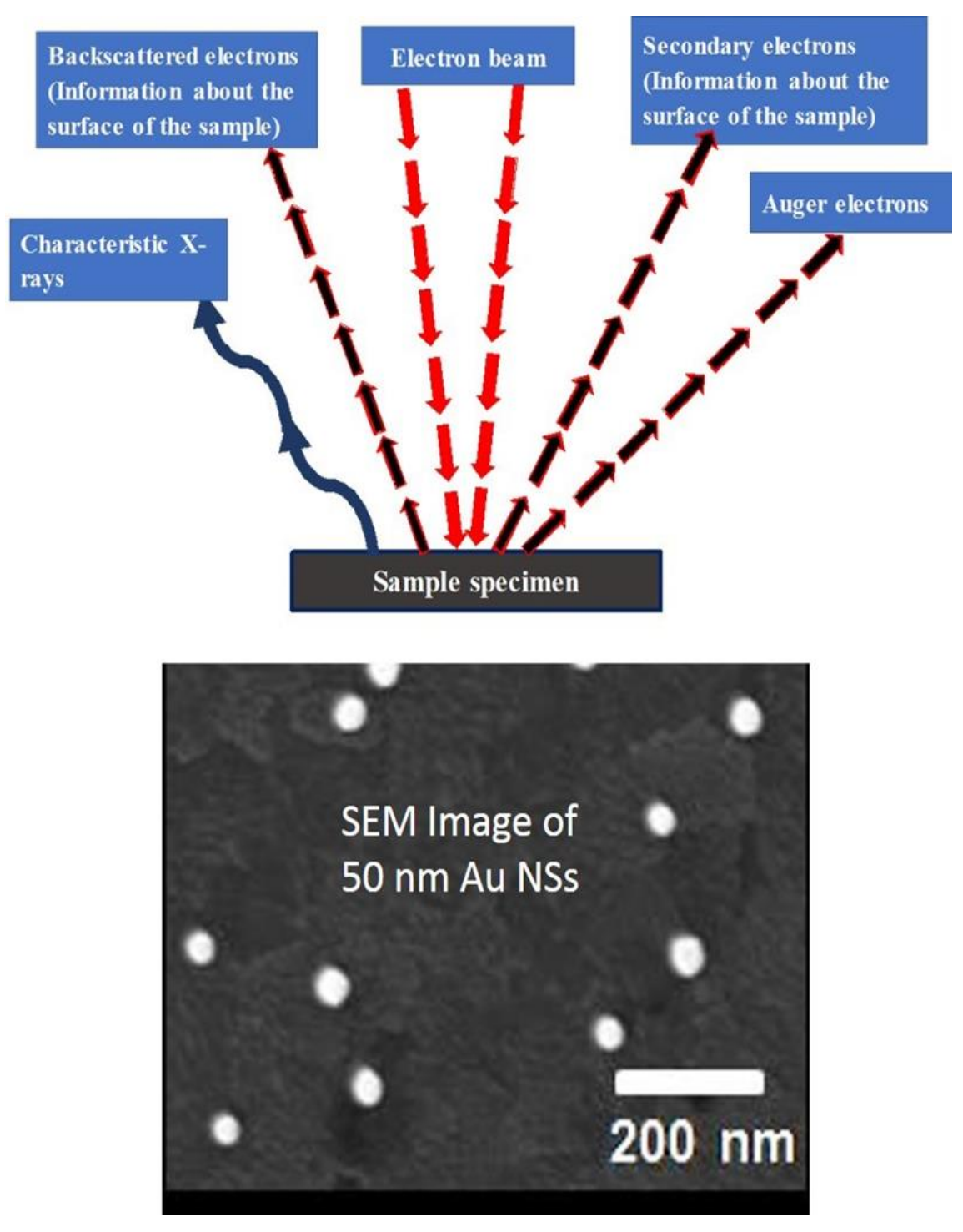

Figure 2. 12. Illustration of the working principle of SEM (top) and a typical SEM image of $50 \mathrm{~nm}$ Au NSs attached to the surface of a glass/ITO electrode (bottom). 
CHAPTER III

\section{SIZE DETERMINATION OF METAL NPS BASED ON ELECTROCHEMICALLY MEASURED SURFACE AREA-TO-VOLUME RATIO}

\subsection{Introduction}

Metal NPs (NPs), especially nanospheres (NSs), have been widely used for many applications such as catalysis, ${ }^{117}$ sensing, ${ }^{118}$ nanoelectronics, ${ }^{119}$ imaging, ${ }^{120}$ therapeutics, ${ }^{121}$ and plasmonics. ${ }^{122}$ For their varied applications, the size of the metal NPs plays a key role. Smaller sized NPs usually show superior performance for catalytic and sensing applications due to their higher SA-to-volume ratio (SA/V), which provides more reactive sites, as compared to bigger sized NPs and bulk materials. Several studies have been performed to analyze the size-dependent oxidation and catalytic, optical and electronic properties of various metals such as $\mathrm{Cu},{ }^{123} \mathrm{Pd},{ }^{124} \mathrm{Au},{ }^{125}$ and $\mathrm{Ag} .{ }^{126}$ Various techniques have been used to determine the size and morphology of metal NPs considering its important relationship with the properties of the NPs.

Some common methods for NP size determination include UV-vis spectroscopy, ${ }^{127}$ Scanning Electron Microscopy (SEM), ${ }^{128}$ Atomic Force Microscopy (AFM), ${ }^{129}$ Transmission Electron Microscopy (TEM), ${ }^{9}$ and Dynamic Light Scattering (DLS). ${ }^{130}$ Despite their wide applicability, all of these methods suffer certain limitations. Electron Microscopy techniques, such as SEM and TEM are expensive, time consuming, and may cause NP aggregation during sample preparation and/or electron beam damage to the sample during imaging. ${ }^{131}$ AFM 
analysis is also fairly expensive, even more time consuming than electron microscopy, and the analysis is not straightforward due to tip convolution and the effect of agglomeration and self-ordering of NPs on rough surfaces. ${ }^{132}$ With DLS, the signal is usually dominated by larger NPs and the analysis can be susceptible to interference from luminescent species. ${ }^{133}$ UV-vis spectroscopy is only useful for plasmonic NPs and only provides a rough estimate of the size.

A search for alternative methods for NP size analysis is useful. Electrochemical methods enjoy the benefit of simple operation, low cost, quick analysis time, and analysis in the native solution state. Several electrochemical strategies for NP size analysis have been recently reported, where NSs are the most common shape. For example, Compton and coworkers used anodic particle coulometry for sizing $\mathrm{Au}^{134}$ and $\mathrm{Ag} \mathrm{NSs}{ }^{135,136}$ by measuring the charge associated with the oxidation of individual NSs as they collide with a microelectrode surface. The monitoring of single NS events is very impressive, but multiple oxidation events for one NS and the aggregation of NSs in solution renders the size analysis somewhat uncertain. ${ }^{137}$ Mega and coworkers ${ }^{138}$ determined the size of Au NSs with very high precision by monitoring an ionic current drop as individual NSs translocated through a nanopore several times. Others have also determined the NS size by monitoring individual NS events based on collisions with microelectrodes ${ }^{18,21,139,140,141}$ or translocation through a nanopore. ${ }^{142,143}$ The peak spacing corresponding to the quantized double-layer charging of MP-AgNSs (Monolayer protected Ag NSs) ${ }^{144}$ in a differential pulse voltammogram correlated to the capacitance, and hence the radius of the NSs. ${ }^{145}$ A decrease in the magnitude of 
surface charge for $\mathrm{Si} \mathrm{NSs}^{59}$ correlated to an increase in the particle size, which reached a constant when the size exceeded a critical value. While these methods are useful and impressive, the techniques using a microelectrode or nanopore require micro or nanofabrication methods, which can be challenging to reproduce. Also, low current measuring potentiostats with fast time scales are needed to detect stochastic events. There are also possibilities of the nanopore becoming blocked by large or aggregated NSs or very small NSs may not be detectable, which sets a size limit to the method. The techniques based on surface charging are sensitive for smaller NSs but then become insensitive to sizes above a critical value, limiting the determination of larger sizes.

Our group previously correlated the oxidation peak potential $\left(E_{p}\right)$ of NSs to their size for an assembly of $\mathrm{Au} \mathrm{NSs}{ }^{86,146}$ ranging from 2-250 nm in diameter and $\mathrm{Ag}$ $\mathrm{NSs}^{85}$ ranging from 8-50 $\mathrm{nm}$ in diameter. The change in $\mathrm{E}_{\mathrm{p}}$ with size is based on theoretical and experimental studies by Henglein ${ }^{147}$ and Plieth, ${ }^{148}$ who predicted a negative shift in the thermodynamic standard reduction potential for metal/metal ion pairs as the metal NS radius decreases. Plieth predicted that the magnitude of the shift is proportional to 1 /radius, which becomes especially large below $5 \mathrm{~nm}$ diameter NSs. The $E_{p}$ also depends on the coverage of the metal NSs on the electrode, making it necessary to keep the coverage in terms of total metal (not number of NSs) constant for these measurements. Our group $31,32-33$ and others ${ }^{149,150,151,152,84}$ measured the oxidation potentials as a function of NS radius, showing some relative agreement with Plieth theory. There are also studies comparing experiment and theory, taking into account diffusion effects and Plieth 
theory. $40,153,154$ It is well accepted that a negative shift in potential occurs with decreasing NS size. Other effects also can play a role, such as the nature of the electrode, local surface charge and aggregation in NPs. ${ }^{82,155-156}$

The measurement of SA/V for NS size analysis as described in this work has many advantages. Compared to microscopy methods, it is inexpensive and fast. In comparison to individual NS collisions ${ }^{21,23-25}$ or pore translocation methods, ${ }^{26,27}$ the technique does not require micro or nanofabrication or highly sensitive or fast current measuring instrumentation. The signal is large and over a longer timescale, but also represents the average of many NSs, so one does not obtain information about individual NSs. Compared to methods correlating the $E_{p}$ from anodic stripping voltammetry to NS size, ${ }^{31,32-33,36,37,38,39}$ the measurement of SA/V is independent of metal NS coverage and it can be used for a much larger range of sizes, where $E_{p}$ is not sensitive to NSs greater than about $40 \mathrm{~nm}$ in diameter. ${ }^{33}$ The electrochemical determination of SA/V of NSs also has benefits over other commonly used methods for SA determination, such as ligand adsorption, ${ }^{157,158}$ BET method, ${ }^{159,160}$ and metal underpotential deposition (UPD). ${ }^{161,75}$ These methods and BET give the SA but not the volume unless you know the sample mass and the density of the material. This would limit you to solid powders that can be weighed out or films whose mass is known. Another issue is that the SA from BET and ligand adsorption methods includes the surfaces that the NSs and the electrode are assembled onto, which could be a source of error.

Our method of determining NS size by measuring SA/V is based on the surface oxidation/reduction and electrochemical dissolution of $\mathrm{Au} .^{162}$ The reduction of 
surface $\mathrm{Au}_{2} \mathrm{O}_{3}$ in acidic solution $\left(0.1 \mathrm{M} \mathrm{HClO}_{4}\right)$ in cyclic voltammetry $(\mathrm{CV})$ is proportional to the SA while anodic stripping voltammetry (ASV) of Au in halide containing acid solutions $\left(0.01 \mathrm{M} \mathrm{KBr}\right.$ in $\left.0.1 \mathrm{M} \mathrm{KClO}_{4}\right)$ is proportional to the entire volume of the NSs. By knowing the SA and the V of the NSs from these voltammetric methods, the SA/V can be calculated electrochemically. Considering the NSs are spherical in shape, the SA/V is expressed as $n\left[4 \pi r^{2}\right] / n\left[(4 / 3) \pi r^{3}\right]=3 / r$, where $\mathrm{n}$ is equal to the number of NSs on the electrode surface and $r$ is the radius of the nanosphere. SA/V is clearly proportional to the NS radius in a mathematically predictable way with a theoretical expected slope of 3 for a plot of $\mathrm{SA} / \mathrm{V}$ versus $1 / \mathrm{r}$. This relation between $\mathrm{SA} / \mathrm{V}$ and radius is successfully used to determine their size electrochemically $(r=3 \mathrm{~V} / \mathrm{SA})$.

\subsection{Experimental Section}

The chemicals, optical characterization, microscopic characterization and electrochemical SA $\mathrm{V}$ assessment are described in the experimental procedures in Chapter II.

3.2.1. Ozone treatment of Au NS samples. Ozone treatment of samples was performed with a Jelight Company Inc. UVO CLEANER model no.42. 


\subsection{Results and Discussion}

The as-synthesized Au NSs were attached to glass/ITO/APTES electrodes to perform electrochemical experiments to determine the SA and V of the Au NSs as shown in Figure 3.1.

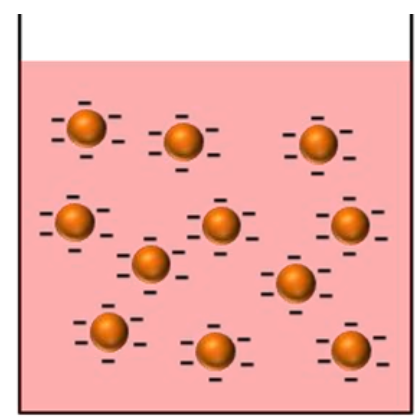

Step 1. Synthesis of NSs

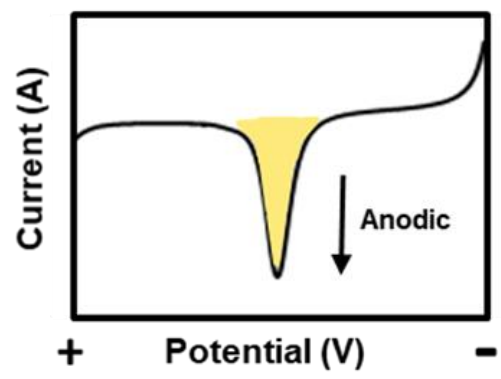

Step 4. Volume measurement of NSs

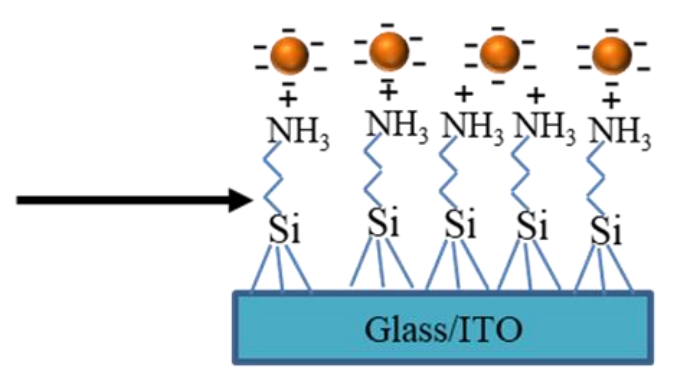

Step 2. Attachment of NSs to Glass/ITOIAPTESElectrode

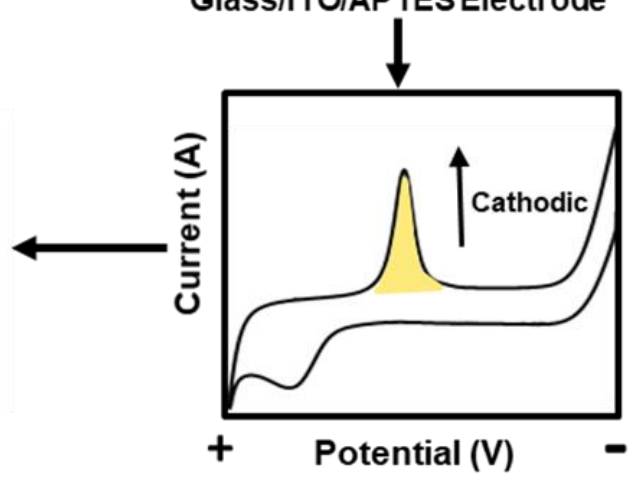

Step 3. Surface areameasurement of NSs

Figure 3. 1. Scheme for general experimental procedure followed in this work.

Since the SA/V is equal to $3 /$ radius ( $r$ ), we expected we would be able to accurately calculate the radius by measuring SA and $V$ electrochemically, where $r$ $=3 \mathrm{~V} / \mathrm{SA}$. As shown in Figure 3.1, citrate-coated Au NSs were synthesized (step 1) and then electrostatically attached to glass/ITO/APTES electrodes by simple soaking (step 2). Next, cyclic voltammetry (CV) was performed on the electrode- 
attached Au NSs in $0.1 \mathrm{M} \mathrm{HClO}_{4}$ solution by scanning from 0.2 to $1.4 \mathrm{~V} \mathrm{vs.} \mathrm{Ag} / \mathrm{AgCl}$ $(3 \mathrm{M} \mathrm{KCl})$. The area of the reduction peak in the $\mathrm{CV}$ (cathodic current up) provides the Coulombs of charge and is proportional to the SA of the Au NSs, as indicated by the yellow highlighted region in step 3 . The same sample was then subjected to oxidative Au stripping in $0.01 \mathrm{M} \mathrm{KBr}$ plus $0.1 \mathrm{M} \mathrm{KClO}_{4}$. The area under the oxidation peak (anodic current down), as indicated by the yellow highlighted region, provides the charge associated with oxidative dissolution of all of the $\mathrm{Au}$ NSs in their entirety, which is proportional to the volume of the NSs (step 4). The oxidation/reduction of Au NSs in $0.1 \mathrm{M} \mathrm{HClO}_{4}$ (reaction 1) and oxidative stripping in $0.01 \mathrm{M} \mathrm{KBr}$ in $0.1 \mathrm{M} \mathrm{KClO}_{4}$ (reactions 2 and 3) occurs by the following reactions. ${ }^{32,51}$

$$
\begin{gathered}
\mathrm{Au}_{2} \mathrm{O}_{3}+6 \mathrm{H}^{+}+6 \mathrm{e}^{-} \leftrightharpoons 2 \mathrm{Au}+3 \mathrm{H}_{2} \mathrm{O} \\
\mathrm{Au}^{0}+4 \mathrm{Br} \longrightarrow \mathrm{AuBr}^{-}+3 \mathrm{e}^{-}\left(\mathrm{E}^{0}=0.85 \mathrm{~V} \text { vs. } \mathrm{NHE}\right) \\
\mathrm{Au}^{0}+2 \mathrm{Br}^{-} \longrightarrow \mathrm{AuBr}^{-}+\mathrm{e}^{-}\left(\mathrm{E}^{0}=0.96 \mathrm{~V} \text { vs. } \mathrm{NHE}\right)
\end{gathered}
$$

However, the three-electron (3e) oxidation is thermodynamically favorable. ${ }^{32} \mathrm{We}$ thus considered reaction 2 (three-electron (3e) oxidation) for oxidative stripping of Au NSs for determining SA/V. We characterized the size of the synthesized $\mathrm{Au}$ NSs by UV-vis spectroscopy and scanning electron microscopy (SEM) as shown in Figure 1. Figure 3.2 A shows the UV-vis spectra normalized for absorbance for the different sized Au NSs. The wavelength of the localized surface plasmon resonance (LSPR) band for $4 \mathrm{~nm}, 15 \mathrm{~nm}, 32 \mathrm{~nm}, 50 \mathrm{~nm}$ and $70 \mathrm{~nm}$ citrate-coated 
Au NSs was located at $505 \mathrm{~nm}, 517 \mathrm{~nm}, 528 \mathrm{~nm}, 536 \mathrm{~nm}$ and $545 \mathrm{~nm}$, respectively. The LSPR band red-shifted with increasing size of Au NSs from $4 \mathrm{~nm}$ to $70 \mathrm{~nm}$, which matches well with the literature. ${ }^{50}$ Figure 3.2B-3.2 F shows SEM images of the different sized Au NSs. The images show that the NSs were well dispersed on
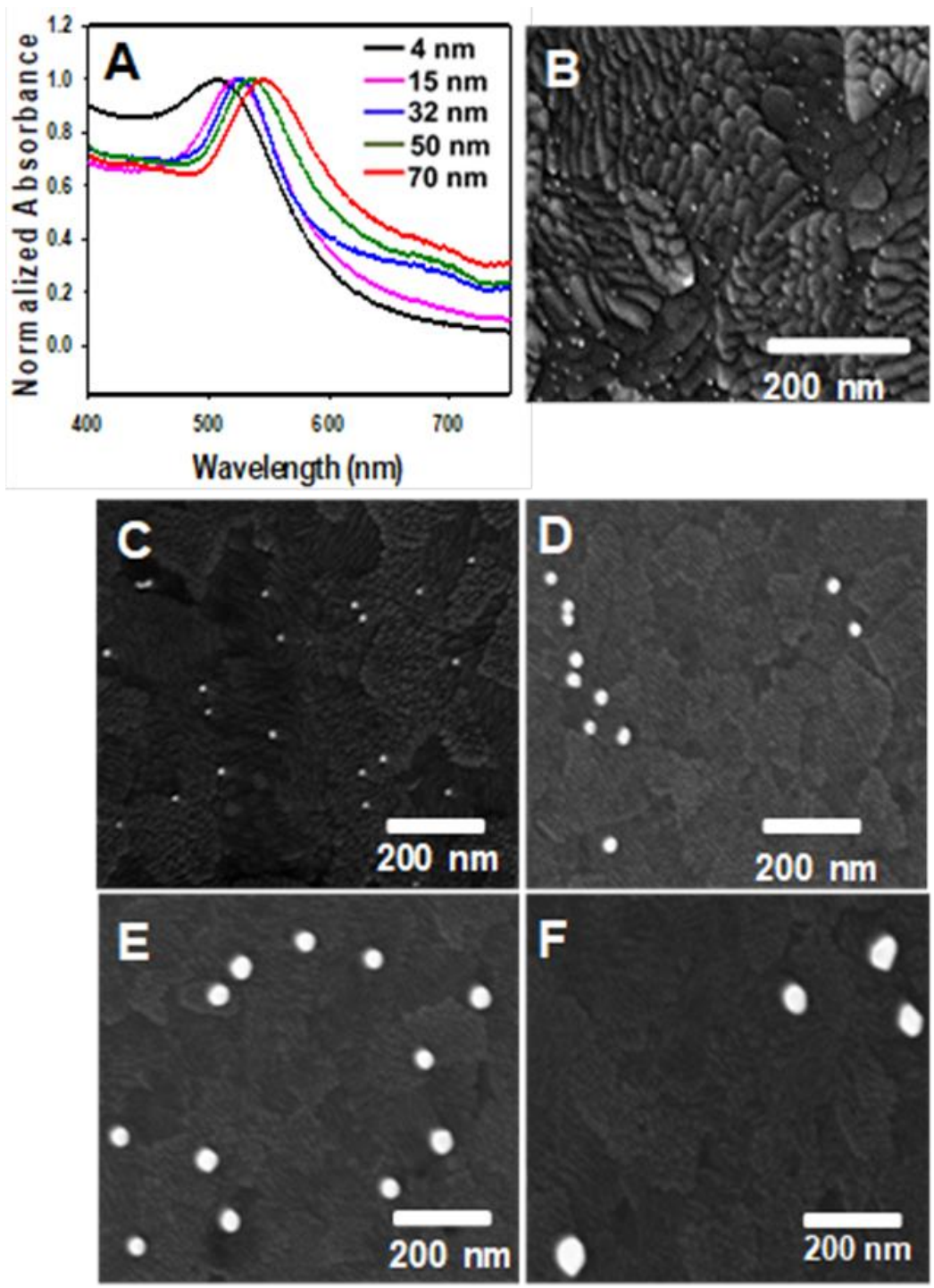

Figure 3. 2. UV-Vis spectra (A) and SEM images (B-F) of $4.1 \pm 0.7,15.1 \pm 1.8$, $31.2 \pm 1.9,50.1 \pm 3.0$ and $70.8 \pm 2.7 \mathrm{~nm}$ citrate coated Au nanospheres. 
the glass/ITO/APTES surface and spherical in shape. The statistical analysis of the NSs from SEM images showed the NSs to be $15.1 \pm 1.8,31.2 \pm 1.9,50.1 \pm$ 3.0, and $70.8 \pm 2.7 \mathrm{~nm}$ in diameter. The $4 \mathrm{~nm}$ sized Au NSs were difficult to analyze by SEM imaging but are of $4.1 \pm 0.7 \mathrm{~nm}$ based on TEM imaging previously reported by our group. ${ }^{32}$

The SA/V ratio was then calculated electrochemically using the procedure in Scheme 1 for the different sized Au NSs. Figure 3.3 A and 3.3 B shows example CVs and ASVs, respectively, for $4 \mathrm{~nm}$ and $15 \mathrm{~nm}$ diameter Au NSs attached to glass/ITO/APTES. The SAs were $1.42 \times 10^{-5} \mathrm{C}$ (Figure $3.3 \mathrm{~A}$, red plot) and $1.46 \mathrm{x}$ $10^{-5} \mathrm{C}$ (Figure $3.3 \mathrm{~A}$, blue plot) for $4 \mathrm{~nm}$ and $15 \mathrm{~nm}$ Au NSs, respectively, while the V was $2.33 \times 10^{-5} \mathrm{C}$ (Figure 3.3. B, red plot) and $6.63 \times 10^{-5} \mathrm{C}$ (Figure $3.3 \mathrm{~B}$, blue plot) for $4 \mathrm{~nm}$ and $15 \mathrm{~nm}$ Au NSs, respectively. The SA was about the same for the $4 \mathrm{~nm}$ and $15 \mathrm{~nm}$ Au NSs in these two samples while the $V$ is much larger for the $15 \mathrm{~nm}$ Au NSs as expected for larger NSs, which have smaller SA/V. The electrochemical SA/V for these two sizes of Au NSs based on the Coulombs of charge for $S A$ and $V$ were $0.640 \pm 0.034$ and $0.230 \pm 0.008$ for $4 \mathrm{~nm}$ and $15 \mathrm{~nm}$ $\mathrm{Au}$ NSs, respectively. The calculated radii using $r=3 \mathrm{~V} / \mathrm{SA}$ were $4.6 \pm 0.2$ and $13.1 \pm 0.6 \mathrm{~nm}$, respectively. 

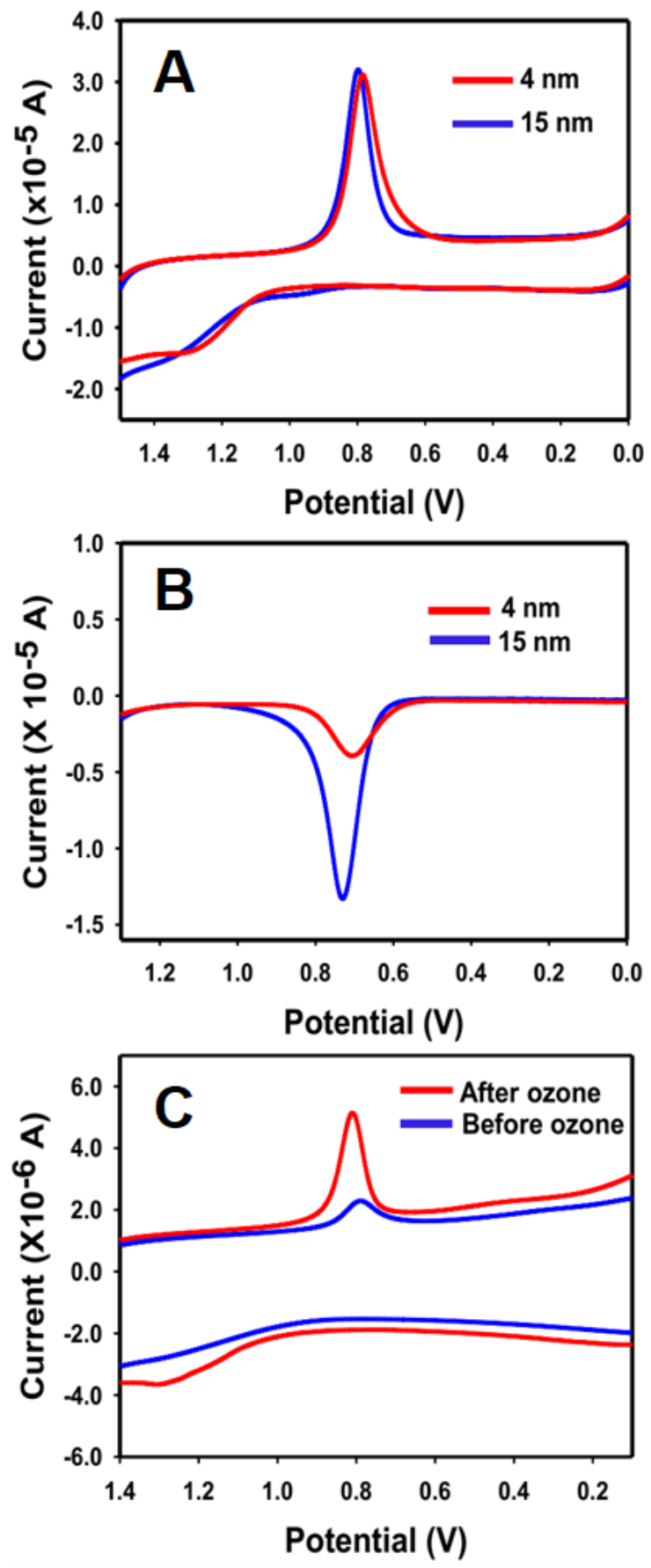

Figure 3. 3. (A) Cyclic voltammograms of glass/ITO/APTES electrodes coated with $4 \mathrm{~nm}$ (red) and $15 \mathrm{~nm}$ (blue) Au NSs in $0.1 \mathrm{M} \mathrm{HClO}_{4}$ showing similar SA based on the $\mathrm{Au}_{2} \mathrm{O}_{3}$ reduction peak near $0.8 \mathrm{~V}$. (B) Anodic stripping voltammograms of the 
same glass/ITO/APTES electrodes in (A) coated with $4 \mathrm{~nm}$ (red) and $15 \mathrm{~nm}$ (blue) Au NSs in $0.01 \mathrm{M} \mathrm{KBr}$ plus $0.1 \mathrm{M} \mathrm{KClO}_{4}$. (C) Cyclic voltammograms of glass/ITO/APTES coated with $50 \mathrm{~nm}$ Au NSs obtained before (blue) and after (red) ozone treatment. We conducted the same type of SA/V and corresponding size analysis for all of the NS sizes in a similar way for each size. The results from 4 trials of each size are provided in Table 3.1.

Table 3.1. SEM measured radius, average electrochemically-measured SA/V, and calculated radius from SA/V for different sizes of Au NSs with and without ozone treatment of the sample.

\begin{tabular}{|c|c|c|c|c|c|}
\hline $\begin{array}{c}\text { No. } \\
\text { of } \\
\text { trials }\end{array}$ & $\begin{array}{c}\text { SEM } \\
\text { measured } \\
\text { radius of } \\
\text { Au NSs }\end{array}$ & $\begin{array}{c}\text { Average } \\
\text { SA/V } \pm \text { Std. } \\
\text { Dev without } \\
\text { ozone } \\
\text { treatment }\end{array}$ & $\begin{array}{c}\text { Calculated } \\
\text { radius from } \\
\text { SA/V without } \\
\text { ozone } \\
\text { treatment }\end{array}$ & $\begin{array}{c}\text { Average } \\
\text { SA/V } \pm \text { Std. } \\
\text { Dev } \\
\text { with ozone }\end{array}$ & $\begin{array}{c}\text { Calculated } \\
\text { radius from } \\
\text { SA/V with } \\
\text { ozone } \\
\text { treatment }\end{array}$ \\
\hline 4 & $\begin{array}{c}2.0 \pm 0.3 \\
\mathrm{~nm}\end{array}$ & $0.640 \pm 0.034$ & $4.7 \pm 0.2$ & $0.75 \pm .0 .018$ & $4.0 \pm 0.1$ \\
\hline 4 & $\begin{array}{c}7.5 \pm 0.9 \\
\mathrm{~nm}\end{array}$ & $0.230 \pm 0.008$ & $13.1 \pm 0.6$ & $\begin{array}{c}0.429 \pm \\
0.030\end{array}$ & $6.9 \pm 1.8$ \\
\hline 4 & $\begin{array}{c}15.6 \pm 0.9 \\
\mathrm{~nm}\end{array}$ & $0.095 \pm 0.012$ & $31.6 \pm 4.2$ & $0.198 \pm$ & $15.2 \pm 1.0$ \\
\hline 4 & $\begin{array}{c}25.1 \pm 1.5 \\
\mathrm{~nm}\end{array}$ & $0.064 \pm 0.007$ & $46.9 \pm 5.2$ & $0.123 \pm$ & $24.4 \pm 3.4$ \\
& & & 0.022 & \\
\hline 4 & $\begin{array}{c}35.4 \pm 1.4 \\
\mathrm{~nm}\end{array}$ & $0.039 \pm 0.007$ & $76.9 \pm 10.2$ & $0.079 \pm$ & $37.9 \pm 10.9$ \\
& & & 0.018 & \\
\hline
\end{tabular}

Also, all the individual trials for both ozone and nonozone treated samples are provided in Tables 3.2 and 3.3. The SA/V clearly decreased with increasing $A u$ NS size as expected but the calculated radii were about a factor of 2 larger than the actual radii determined by SEM (see columns 2 and 4). 
Table 3. 2. SEM measured radius of the nanospheres (NSs) (average \pm std. dev.), charge under the oxide reduction peak and the charge under the anodic stripping peaks, SA/V (average \pm std. dev.) and calculated radius from SA/V for different sized Au NSs without ozone treatment.

\begin{tabular}{|c|c|c|c|c|c|}
\hline $\begin{array}{c}\text { SEM } \\
\text { Measured } \\
\text { Radius of } \\
\text { Au NSs } \\
(\mathbf{n m})\end{array}$ & $\begin{array}{c}\text { CV } \\
\text { Measured } \\
\text { SA } \\
\text { (Coulombs) }\end{array}$ & $\begin{array}{c}\text { ASV } \\
\text { Measured } \\
\text { Volume } \\
\text { (Coulombs) }\end{array}$ & $\begin{array}{c}\text { SA-to- } \\
\text { Volume } \\
\text { Ratio } \\
\text { (SA/V) }\end{array}$ & $\begin{array}{c}\text { Average } \\
\text { SA/V } \pm \\
\text { Std. Dev. }\end{array}$ & $\begin{array}{c}\text { Calculated } \\
\text { Radius } \\
\text { from SA/V } \\
\text { Without } \\
\text { Ozone } \\
\text { (nm) }\end{array}$ \\
\hline \multirow[t]{4}{*}{$2.1 \pm 0.4$} & $6.76 \times 10^{-6}$ & $1.11 \times 10^{-5}$ & 0.610 & \multirow[t]{4}{*}{$\begin{array}{c}0.640 \pm \\
0.034\end{array}$} & \multirow[t]{4}{*}{$4.7 \pm 0.2$} \\
\hline & $4.38 \times 10^{-5}$ & $6.36 \times 10^{-5}$ & 0.689 & & \\
\hline & $5.47 \times 10^{-5}$ & $8.75 \times 10^{-5}$ & 0.625 & & \\
\hline & $9.77 \times 10^{-5}$ & $1.50 \times 10^{-4}$ & 0.651 & & \\
\hline \multirow[t]{4}{*}{$7.5 \pm 0.9$} & $4.97 \times 10^{-5}$ & $2.19 \times 10^{-4}$ & 0.227 & \multirow[t]{4}{*}{$\begin{array}{c}0.230 \pm \\
0.008\end{array}$} & \multirow[t]{4}{*}{$13.1 \pm 0.6$} \\
\hline & $2.01 \times 10^{-6}$ & $9.23 \times 10^{-6}$ & 0.217 & & \\
\hline & $1.46 \times 10^{-5}$ & $6.29 \times 10^{-5}$ & 0.231 & & \\
\hline & $1.96 \times 10^{-5}$ & $8.06 \times 10^{-5}$ & 0.242 & & \\
\hline \multirow[t]{4}{*}{$15.6 \pm 0.9$} & $6.63 \times 10^{-6}$ & $6.44 \times 10^{-5}$ & 0.103 & \multirow[t]{4}{*}{$\begin{array}{c}0.095 \pm \\
0.012\end{array}$} & \multirow[t]{4}{*}{$31.6 \pm 4.2$} \\
\hline & $1.54 \times 10^{-5}$ & $1.42 \times 10^{-4}$ & 0.108 & & \\
\hline & $2.48 \times 10^{-6}$ & $2.85 \times 10^{-5}$ & 0.087 & & \\
\hline & $5.31 \times 10^{-6}$ & $6.51 \times 10^{-5}$ & 0.082 & & \\
\hline
\end{tabular}




\begin{tabular}{|c|c|c|c|c|c|}
\hline \multirow{4}{*}{$\begin{array}{l}25.1 \pm \\
1.5\end{array}$} & $1.89 \times 10^{-5}$ & $3.86 \times 10^{-5}$ & 0.049 & \multirow{4}{*}{$\begin{array}{c}0.064 \pm \\
0.007\end{array}$} & \multirow[t]{4}{*}{$46.9 \pm 5.2$} \\
\hline & $4.11 \times 10^{-5}$ & $1.01 \times 10^{-3}$ & 0.041 & & \\
\hline & $1.84 \times 10^{-5}$ & $4.62 \times 10^{-4}$ & 0.039 & & \\
\hline & $1.26 \times 10^{-5}$ & $3.56 \times 10^{-4}$ & 0.035 & & \\
\hline \multirow{3}{*}{$\begin{array}{l}35.4 \pm \\
1.4\end{array}$} & $1.89 \times 10^{-5}$ & $3.86 \times 10^{-4}$ & 0.049 & \multirow{3}{*}{$\begin{array}{c}0.039 \pm \\
0.007\end{array}$} & \multirow[t]{3}{*}{$76.9 \pm 10.2$} \\
\hline & $4.11 \times 10^{-5}$ & $1.01 \times 10^{-3}$ & 0.041 & & \\
\hline & $1.84 \times 10^{-5}$ & $4.62 \times 10^{-4}$ & 0.039 & & \\
\hline
\end{tabular}


Table 3. 5. SEM measured radius of the NSs (average \pm std. dev.), charge under the oxide reduction peak and the charge under the anodic stripping peaks, SA/V (average \pm std. dev.) and calculated radius from SA/V for different sized Au NSs with ozone treatment.

\begin{tabular}{|c|c|c|c|c|c|c|}
\hline $\begin{array}{c}\text { SEM } \\
\text { Measu } \\
\text { red } \\
\text { Radiu } \\
\text { s of } \\
\text { Au } \\
\text { NSs } \\
(\mathbf{n m})\end{array}$ & $\begin{array}{c}\text { CV } \\
\text { Measured } \\
\text { SA Before } \\
\text { Ozone } \\
\text { (Coulombs) }\end{array}$ & $\begin{array}{c}\text { CV } \\
\text { Measured } \\
\text { SA After } \\
\text { Ozone } \\
\text { (Coulombs } \\
\text { ) }\end{array}$ & $\begin{array}{c}\text { ASV } \\
\text { Measured } \\
\text { Volume } \\
\text { (Coulomb } \\
\text { s) }\end{array}$ & $\begin{array}{c}\text { SA-to- } \\
\text { Volume } \\
\text { Ratio } \\
\text { (SA/V) }\end{array}$ & $\begin{array}{l}\text { Avera } \\
\text { ge } \\
\text { SA/V } \\
\pm \text { Std. } \\
\text { Dev. }\end{array}$ & $\begin{array}{c}\text { Calcula } \\
\text { ted } \\
\text { Radius } \\
\text { from } \\
\text { SA/V } \\
\text { With } \\
\text { Ozone } \\
\text { (nm) }\end{array}$ \\
\hline \multirow[t]{4}{*}{$\begin{array}{c}2.0 \pm \\
0.4\end{array}$} & $4.01 \times 10^{-5}$ & $4.93 \times 10^{-5}$ & $6.63 \times 10^{-5}$ & 0.743 & \multirow[t]{4}{*}{$\begin{array}{c}0.75 \pm \\
0.02\end{array}$} & \multirow[t]{4}{*}{$\begin{array}{c}4.0 \pm \\
0.1\end{array}$} \\
\hline & $3.41 \times 10^{-5}$ & $5.35 \times 10^{-5}$ & $7.39 \times 10^{-5}$ & 0.723 & & \\
\hline & $3.04 \times 10^{-5}$ & $3.47 \times 10^{-5}$ & $4.52 \times 10^{-5}$ & 0.767 & & \\
\hline & $3.40 \times 10^{-5}$ & $3.73 \times 10^{-5}$ & $4.90 \times 10^{-5}$ & 0.761 & & \\
\hline \multirow{4}{*}{$\begin{array}{c}7.5 \pm \\
0.9\end{array}$} & $1.17 \times 10^{-6}$ & $3.35 \times 10^{-6}$ & $9.87 \times 10^{-6}$ & 0.339 & \multirow{4}{*}{$\begin{array}{c}0.429 \\
\pm \\
0.030\end{array}$} & \multirow{4}{*}{$\begin{array}{c}6.9 \pm \\
1.8\end{array}$} \\
\hline & $1.17 \times 10^{-6}$ & $9.23 \times 10^{-6}$ & $1.25 \times 10^{-5}$ & 0.439 & & \\
\hline & $1.86 \times 10^{-6}$ & $6.29 \times 10^{-5}$ & $1.52 \times 10^{-5}$ & 0.557 & & \\
\hline & $1.71 \times 10^{-6}$ & $8.06 \times 10^{-5}$ & $4.61 \times 10^{-5}$ & 0.329 & & \\
\hline \multirow{4}{*}{$\begin{array}{c}15.6 \pm \\
0.9\end{array}$} & $1.82 \times 10^{-6}$ & $9.66 \times 10^{-6}$ & $4.63 \times 10^{-5}$ & 0.209 & \multirow{4}{*}{$\begin{array}{c}0.198 \\
\pm \\
0.020\end{array}$} & \multirow[t]{4}{*}{$\begin{array}{c}15.2 \pm \\
1.0\end{array}$} \\
\hline & $1.86 \times 10^{-5}$ & $3.41 \times 10^{-5}$ & $1.57 \times 10^{-4}$ & 0.217 & & \\
\hline & $1.75 \times 10^{-5}$ & $3.62 \times 10^{-5}$ & $1.96 \times 10^{-5}$ & 0.185 & & \\
\hline & $3.79 \times 10^{-5}$ & $5.15 \times 10^{-5}$ & $2.51 \times 10^{-4}$ & 0.206 & & \\
\hline
\end{tabular}




\begin{tabular}{|c|c|c|c|c|c|c|}
\hline $\begin{array}{c}25.1 \pm \\
1.5\end{array}$ & $8.74 \times 10^{-7}$ & $4.26 \times 10^{-6}$ & $2.74 \times 10^{-5}$ & 0.155 & $0.123 \pm$ & $24.4 \pm$ \\
& & & & & 0.022 & 3.4 \\
\cline { 2 - 5 } & $7.97 \times 10^{-7}$ & $2.46 \times 10^{-6}$ & $2.15 \times 10^{-5}$ & 0.114 & & \\
\cline { 2 - 5 } & $2.11 \times 10^{-5}$ & $2.67 \times 10^{-5}$ & $2.55 \times 10^{-5}$ & 0.105 & & \\
\cline { 2 - 5 } & $2.66 \times 10^{-5}$ & $5.69 \times 10^{-5}$ & $4.89 \times 10^{-4}$ & 0.116 & & \\
\hline $\begin{array}{c}35.4 \pm \\
1.4\end{array}$ & $7.70 \times 10^{-7}$ & $2.97 \times 10^{-6}$ & $3.05 \times 10^{-5}$ & 0.097 & $\begin{array}{c}0.079 \pm \\
0.018\end{array}$ & $\begin{array}{c}37.9 \pm \\
10.9\end{array}$ \\
\cline { 2 - 5 } & $2.09 \times 10^{-5}$ & $4041 \times 10^{-5}$ & $8.19 \times 10^{-4}$ & 0.054 & & \\
\cline { 2 - 5 } & $2.39 \times 10^{-6}$ & $3.73 \times 10^{-6}$ & $4.27 \times 10^{-5}$ & 0.087 & & \\
\cline { 2 - 5 } & $8.97 \times 10^{-6}$ & $6.31 \times 10^{-5}$ & $8.10 \times 10^{-4}$ & 0.078 & & \\
\hline
\end{tabular}

We believed that the discrepancy between the SEM radius and electrochemicallydetermined radius was due to a smaller SA value than the true value due to impurities or adsorbed citrate on the Au NS surfaces. This would block the surface Au from full oxidation, leading to a smaller SA value than the true geometric value. We tested this idea by measuring the $\mathrm{CV}$ of the Au NSs in $0.1 \mathrm{M} \mathrm{HClO}_{4}$ before and after exposing the glass/ITO/APTES/Au NSs to ozone. Ozone has been successfully used previously to remove adsorbed ligands from NPs in order to expose the maximum number of surface $\mathrm{Au}$ atoms to the reaction conditions. ${ }^{163}$ Figure $3.3 \mathrm{C}$ shows a CV measured on glass/ITO/APTES coated with $50 \mathrm{~nm}$ diameter Au NSs before (blue) and after (red) ozone treatment for 30-40 minutes. The CV clearly shows a significant increase in the SA from $2.4 \times 10^{-6} \mathrm{C}$ before to $5.3 \times 10^{-6} \mathrm{C}$ after ozone treatment based on the increased peak area of the $\mathrm{Au}_{2} \mathrm{O}_{3}$ reduction peak at $0.8 \mathrm{~V}$. The same behavior occurred for all other Au NS sizes prepared in this work. Figure 3.4 show the CVs for effect of ozone on $4 \mathrm{~nm}$ and 15 nm Au NSs. The corresponding SA/V values went up and the electrochemically- 
determined size of the different Au NSs better matched the size determined by SEM for the 15, 32, 50 and $70 \mathrm{~nm}$ Au NSs.
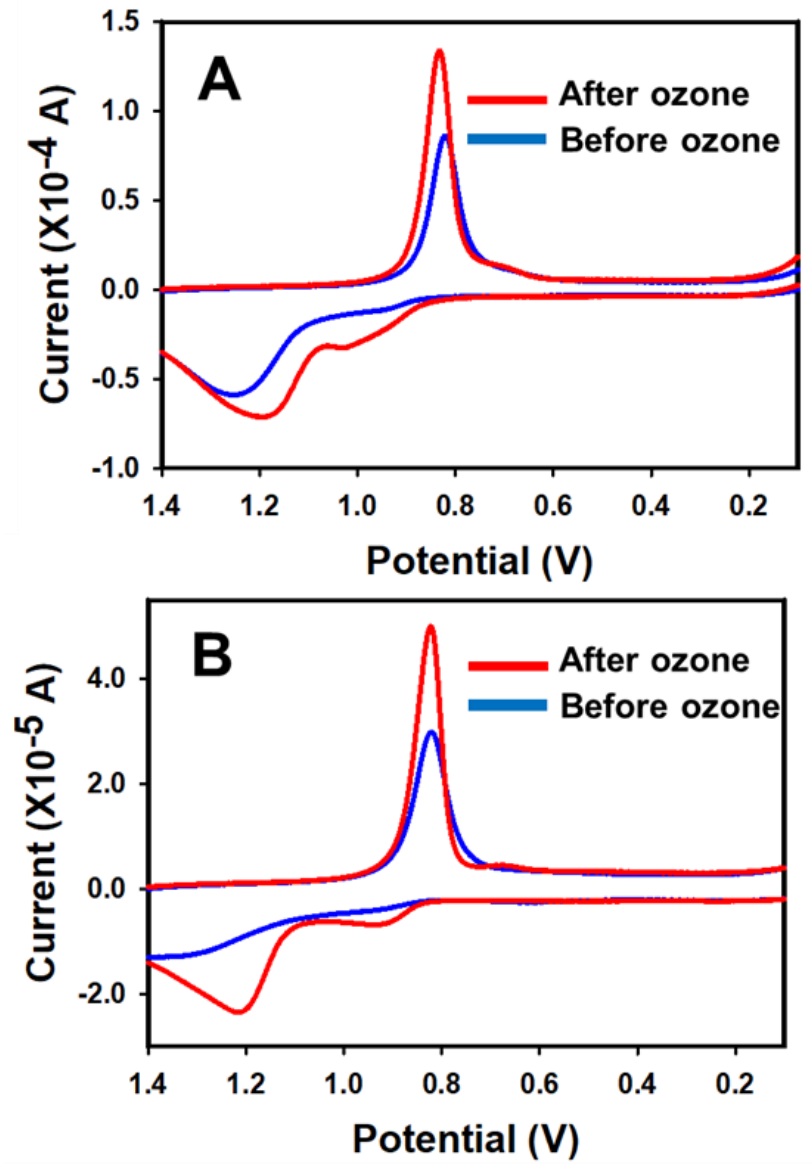

Figure 3. 4. Cyclic voltammograms of glass/ITO/APTES coated with $4 \mathrm{~nm}(\mathrm{~A})$ and $15 \mathrm{~nm}$ (B) Au NSs obtained before (blue) and after (red) ozone treatment. 
Table 1 above also provides the SA/V ratios and electrochemically-measured radius for all Au NSs before (column 4) and after treatment with ozone (column 6) as compared to the SEM determined radius (column 2). In all cases the radius determined from the electrochemically measured SA/V ratio was closer to the SEM determined radius for the Au NSs that were treated with ozone. This is likely due to the removal of impurities blocking the Au NS surface, which gave a larger and more accurate measurement of SA. These same impurities (or possibly adsorbed citrate) do not affect the electrochemically measured V. The only sample with significant error in the electrochemically-measured radius even after ozone treatment was the $4 \mathrm{~nm}$ diameter Au NSs $(4.0 \mathrm{~nm}$ radius predicted vs. $2.0 \mathrm{~nm}$ radius actual). We believe this may be due to ozone induced ripening of the $4 \mathrm{~nm}$ Au NSs to produce larger sizes or due to the $4 \mathrm{~nm}$ Au NSs being somewhat buried in the glass/ITO/APTES electrode surface, which reduces its accessible SA, even though it is clean of impurities. We are currently testing these two hypotheses. We also believe that these variations for $4 \mathrm{~nm}$ Au NSs are possibly due to more thickness of the citrate bilayer shell structure at the citrate/Au interface..$^{164}$

We also plotted the electrochemically-determined SA/V as function of $1 /$ radius using the SEM measured radius of the Au NSs as shown in Figure 3.5. The blue plot of SA/V vs $1 /$ radius was without ozone treatment, which was linear with a slope of 1.8. While the radius could be accurately predicted from the SA/V using this 
calibration curve, the slope was significantly lower than the theoretically expected value of 3 for a spherical shape, again due to adsorbed impurities or citrate. The

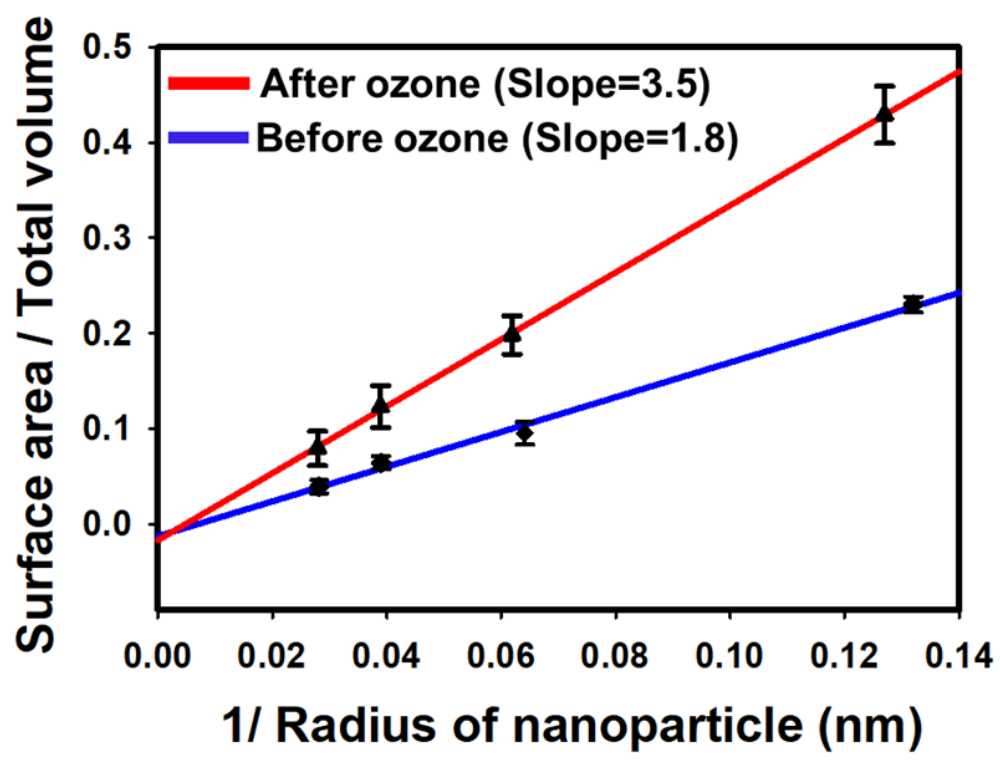

Figure 3. 5. Plot of experimentally-measured $S A / V$ as a function of $1 /$ radius as measured by SEM for 15, 32, 50 and $70 \mathrm{~nm}$ diameter citrate-coated Au NPs. The SA was measured in $0.1 \mathrm{M} \mathrm{HClO}_{4}$ and $\mathrm{V}$ was measured in $0.01 \mathrm{M} \mathrm{KBr}$ in $0.1 \mathrm{M}$ $\mathrm{KClO}_{4}$ without ozone treatment (blue) and with ozone treatment (red).

red plot in Figure 4 shows the same plot for the same size Au NSs but after ozone treatment. Following ozone, the plot of SA/V vs $1 / \mathrm{r}$ was linear with a slope of 3.5 , which is in much better agreement with the expected value of 3 for a sphere.

In this work we also wanted to test the effect of Au NS surface coverage on the measured SA/V ratio and the determined radius in order to determine if the analysis only works over a certain coverage range. In these experiments, we 
varied the coverage of $4 \mathrm{~nm}$ and $15 \mathrm{~nm}$ Au NSs on the electrode surface from 10${ }^{6}$ to $10^{-4} \mathrm{C}$ and measured the SA/V of the samples. We then made a plot of the electrochemically determined SA/V versus the coverage, determined by the ASV peak area. Figure 3.6 below and Table 3.4 and shows the corresponding results for the two sizes studied. The data show that the SA/V remains fairly constant with a minimal decrease at the highest $10^{-4} \mathrm{C}$ coverage for the $4 \mathrm{~nm}$ Au NSs while there is a bigger decrease in the SA/V for $15 \mathrm{~nm}$ Au NSs as the Au NS coverage increased to $10^{-4} \mathrm{C}$.

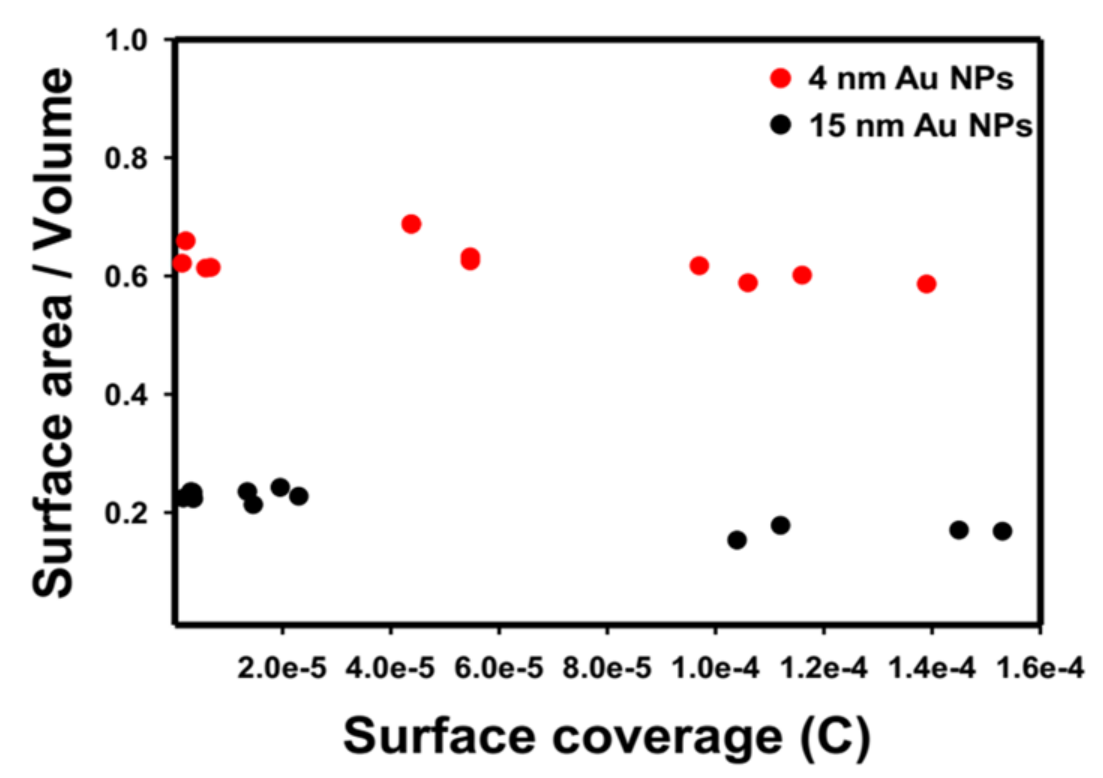

Figure 3. 6. Effect of coverage on SA/V for $4 \mathrm{~nm}$ (red) and $15 \mathrm{~nm}$ (black) Au NPs. 
Table 3. 7. Effect of coverage on SA/V for $4 \mathrm{~nm}$ and $15 \mathrm{~nm}$ Au NPs.

\begin{tabular}{|c|c|c|c|}
\hline $\begin{array}{l}\text { Au NPs } \\
\text { size }\end{array}$ & $\begin{array}{l}\text { Number } \\
\text { of trials }\end{array}$ & $\begin{array}{l}\text { CV surface } \\
\text { coverages } \\
\text { (Coulombs) }\end{array}$ & $\begin{array}{c}\text { Average ratio } \pm \\
\text { Std. Dev }\end{array}$ \\
\hline \multirow{12}{*}{$4 \mathrm{~nm}$} & \multirow{4}{*}{4} & $0.97 \times 10^{-4}$ & \multirow{4}{*}{$0.598 \pm 0.014$} \\
\hline & & $1.16 \times 10^{-4}$ & \\
\hline & & $1.06 \times 10^{-4}$ & \\
\hline & & $1.39 \times 10^{-4}$ & \\
\hline & \multirow{4}{*}{4} & $5.47 \times 10^{-5}$ & \multirow{4}{*}{$0.658 \pm 0.034$} \\
\hline & & $4.38 \times 10^{-5}$ & \\
\hline & & $4.38 \times 10^{-5}$ & \\
\hline & & $5.47 \times 10^{-5}$ & \\
\hline & \multirow{4}{*}{4} & $2.13 \times 10^{-6}$ & \multirow{4}{*}{$0.626 \pm 0.021$} \\
\hline & & $6.76 \times 10^{-6}$ & \\
\hline & & $5.83 \times 10^{-6}$ & \\
\hline & & $1.46 \times 10^{-6}$ & \\
\hline \multirow{12}{*}{$15 \mathrm{~nm}$} & \multirow{4}{*}{4} & $1.53 \times 10^{-4}$ & \multirow{4}{*}{$0.167 \pm 0.010$} \\
\hline & & $1.12 \times 10^{-4}$ & \\
\hline & & $1.04 \times 10^{-4}$ & \\
\hline & & $1.45 \times 10^{-4}$ & \\
\hline & \multirow{4}{*}{4} & $1.46 \times 10^{-5}$ & \multirow{4}{*}{$0.234 \pm 0.006$} \\
\hline & & $1.96 \times 10^{-5}$ & \\
\hline & & $2.30 \times 10^{-5}$ & \\
\hline & & $1.35 \times 10^{-5}$ & \\
\hline & \multirow{4}{*}{4} & $1.69 \times 10^{-6}$ & \multirow{4}{*}{$0.227 \pm 0.005$} \\
\hline & & $3.08 \times 10^{-6}$ & \\
\hline & & $3.08 \times 10^{-6}$ & \\
\hline & & $3.42 \times 10^{-6}$ & \\
\hline
\end{tabular}

Table 3.5 below shows all the individual values for the two sizes studied. 
Table 3. 8. SA (charge under the oxide reduction peak) and total volume (charge under the anodic stripping peaks), SA $/$ (average \pm std. dev.) at different coverages $\left(10^{-4}, 10^{-5}\right.$ and $\left.10^{-6}\right)$ for $4 \mathrm{~nm}$ and $15 \mathrm{~nm}$ Au NSs.

\begin{tabular}{|c|c|c|c|c|c|}
\hline $\begin{array}{c}\text { Au NSs } \\
\text { Diameter } \\
\text { (nm) }\end{array}$ & $\begin{array}{c}\text { ASV } \\
\text { Measured } \\
\text { Volume in } \\
\text { Order of } \\
\text { Magnitude } \\
\text { (Coulombs) }\end{array}$ & $\begin{array}{c}\text { CV } \\
\text { Measured } \\
\text { SA } \\
\text { (Coulombs) }\end{array}$ & $\begin{array}{c}\text { ASV } \\
\text { Measured } \\
\text { Volume } \\
\text { (Coulombs) }\end{array}$ & $\begin{array}{c}\text { SA-to- } \\
\text { Volume } \\
\text { Ratio } \\
\text { (SA/V) }\end{array}$ & $\begin{array}{c}\text { Average } \\
\text { SA/V } \pm \\
\text { Std. } \\
\text { Dev. }\end{array}$ \\
\hline \multirow{12}{*}{4.2} & \multirow{4}{*}{$10^{-4}$} & $0.97 \times 10^{-4}$ & $1.58 \times 10^{-4}$ & 0.617 & \multirow{4}{*}{$\begin{array}{c}0.598 \pm \\
0.014\end{array}$} \\
\hline & & $1.16 \times 10^{-4}$ & $1.93 \times 10^{-4}$ & 0.601 & \\
\hline & & $1.06 \times 10^{-4}$ & $1.80 \times 10^{-4}$ & 0.588 & \\
\hline & & $1.39 \times 10^{-4}$ & $2.37 \times 10^{-4}$ & 0.586 & \\
\hline & \multirow{4}{*}{$10^{-5}$} & $5.47 \times 10^{-5}$ & $8.64 \times 10^{-5}$ & 0.632 & \multirow[t]{4}{*}{$\begin{array}{c}0.658 \pm \\
0.034\end{array}$} \\
\hline & & $4.38 \times 10^{-5}$ & $6.37 \times 10^{-5}$ & 0.687 & \\
\hline & & $4.38 \times 10^{-5}$ & $6.36 \times 10^{-5}$ & 0.688 & \\
\hline & & $5.47 \times 10^{-5}$ & $8.75 \times 10^{-5}$ & 0.625 & \\
\hline & \multirow{3}{*}{$10^{-6}$} & $2.13 \times 10^{-6}$ & $3.23 \times 10^{-6}$ & 0.659 & \multirow{3}{*}{$\begin{array}{c}0.626 \\
\pm 0.021\end{array}$} \\
\hline & & $6.76 \times 10^{-6}$ & $1.10 \times 10^{-6}$ & 0.614 & \\
\hline & & $1.46 \times 10^{-6}$ & $2.35 \times 10^{-6}$ & 0.621 & \\
\hline & & $5.83 \times 10^{-6}$ & $9.51 \times 10^{-6}$ & 0.613 & \\
\hline \multirow{4}{*}{15.6} & \multirow{4}{*}{$10^{-4}$} & $1.53 \times 10^{-4}$ & $9.08 \times 10^{-4}$ & 0.168 & \multirow[t]{4}{*}{$\begin{array}{c}0.167 \pm \\
0.010\end{array}$} \\
\hline & & $1.12 \times 10^{-4}$ & $6.27 \times 10^{-4}$ & 0.178 & \\
\hline & & $1.04 \times 10^{-4}$ & $6.77 \times 10^{-4}$ & 0.153 & \\
\hline & & $1.45 \times 10^{-4}$ & $8.51 \times 10^{-4}$ & 0.170 & \\
\hline
\end{tabular}




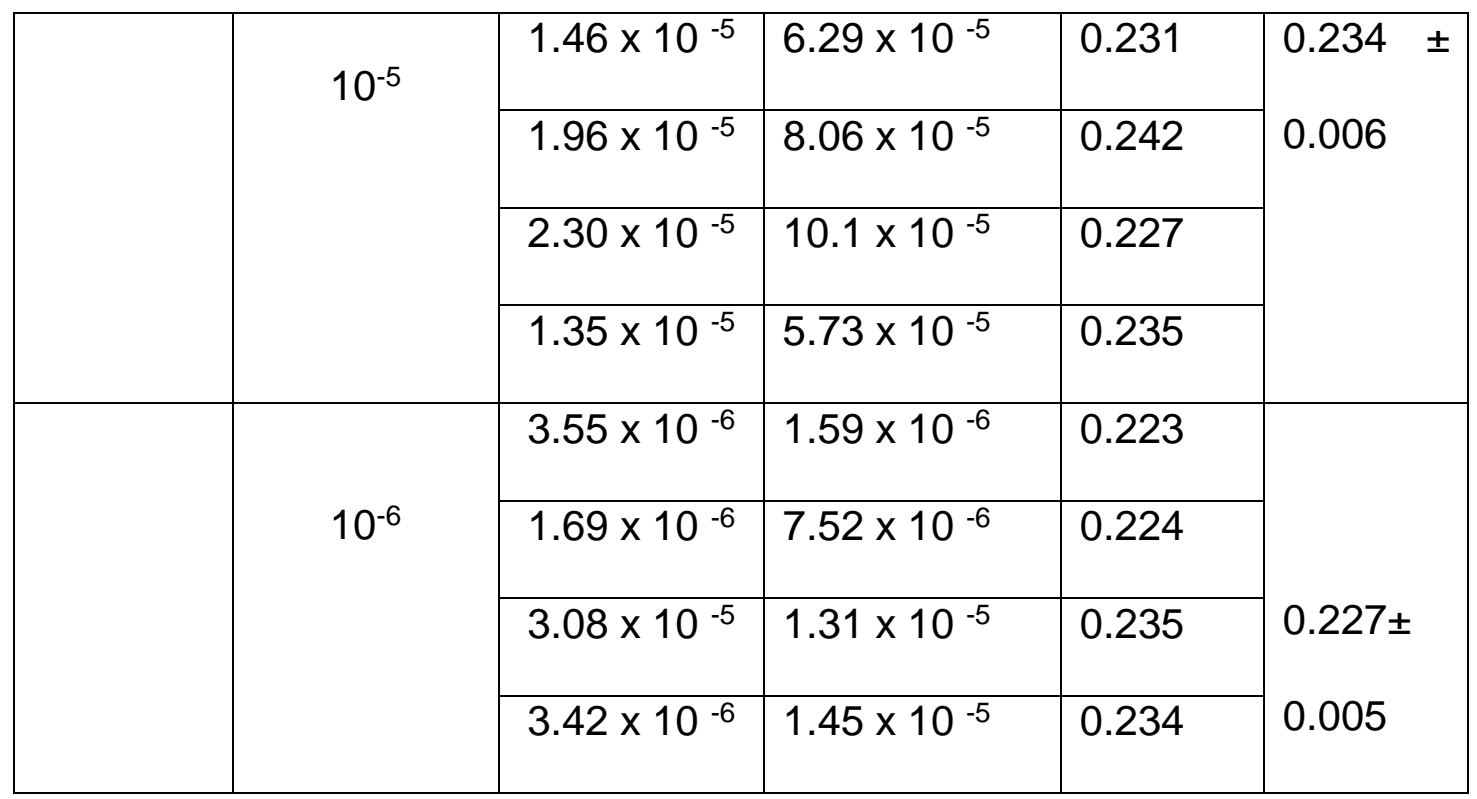

This decreased SA/V is due to contact (or aggregation) between the Au NSs at higher coverage. Contact between aggregated NSs makes them effectively larger and reduces the exposed SA. This would lead to a smaller SA/V than for individual, well-spaced NSs and lead to an error in the radius calculation. For size analysis, it is best to use low to moderate coverages of Au NSs on the electrode surface that are $10^{-5} \mathrm{C}$ or less.

\subsection{Conclusions}

Here we described the electrochemical measurement of SA/V of different sized Au NSs for calculation of the NS radius. Citrate-coated Au NSs of different size were electrostatically attached to glass/ITO/APTES. A plot of SA/V as a function of $1 /$ radius of $4,15,32,50$ and $70 \mathrm{~nm}$ diameter Au NPs was linear with a slope of 
3.5 when the Au NSs were treated with ozone first and the SA determined in 0.1 $\mathrm{M} \mathrm{HClO}_{4}$ using $\mathrm{CV}$ and the $\mathrm{V}$ determined in $0.01 \mathrm{M} \mathrm{KBr}$ plus $0.1 \mathrm{M} \mathrm{KClO}_{4}$ using ASV. The size of the Au NSs determined by our method is very close to the size determined by SEM imaging for all sizes except for the $4 \mathrm{~nm}$ diameter Au NSs, verifying good accuracy of our method. Our approach is a simple, cheap alternative to other methods for metal NS size analysis and the SA/V measurement could also be correlated to the reactivity and other important fundamental properties of metal NSs in the future. 


\section{CHAPTER IV}

\section{ELECTROCHEMICAL SURFACE AREA-TO-VOLUME RATIO (SA/V) OF DIFFERENT AGGREGATED STATES OF AU NSS}

\subsection{Introduction}

Aggregated NPs have recently gained significant attention due to their useful applications and advantages over their non-aggregated counterparts for certain applications. Some of the examples include improved catalytic activity observed in case of partially aggregated Au NPs towards photocatalytic conversion of 4-NP observed by Kim and coworkers ${ }^{89}$, enhanced fluorescence signal observed for Cy5-labelled oligonucleotides sandwiched between aggregated Ag NPs (dimers) by Lakowicz_and coworkers ${ }^{91}$ and use of Au NPs dimers having a recognition site for a specific DNA-binding protein by Bonham and coworkers $^{94}$ to study DNA-protein interactions. Considering such useful applications aggregated NPs are being routinely analyzed by different analytical techniques such as UV vis spectroscopy, ${ }^{165-166}$, Raman spectroscopy ${ }^{167}$ and different microsocpic ${ }^{168}$ techniques.

Electrochemical analysis of aggregated NPs can be a good alternative to these available methods. Our group has successfully employed ASV to analyze aggregated $4 \mathrm{~nm}$ and $15 \mathrm{~nm}$ Au NPs. ${ }^{156}$ In this work, we aim to determine the $\mathrm{SA} / \mathrm{V}$ of the aggregated forms of $4 \mathrm{~nm}$ and $15 \mathrm{~nm}$ Au NPs using the similar approach as described in experimental section in Chapter II. SA contribution in 
case of SA/V using our electrochemical method can also be useful to know besides the volume of NPs as observed in ASV.

\subsection{Experimental Section}

The chemicals, optical characterization, microscopic characterization and electrochemical SA/V assessment are described in the experimental procedures in Chapter II.

\subsection{Results and Discussion}

Figure 4.1 (Frame A) shows cyclic voltammograms of glass/ITO/APTES/Au NPs $(4 \mathrm{~nm})$ obtained in $0.1 \mathrm{M} \mathrm{HClO}_{4}$ from $-0.2 \mathrm{~V}$ to $1.6 \mathrm{~V}$. The $4 \mathrm{~nm}$ Au NPs were attached to the glass/ITO/APTES after sitting at $\mathrm{pH} 8.3$ and at $\mathrm{pH} 3.1$ overnight, corresponding to non-aggregated (blue plot) and aggregated (orange plot) Au NPs, respectively. The anodic peak near $1.3 \mathrm{~V}$ in Frame $\mathrm{A}$ corresponds to surface oxidation of $\mathrm{Au}$ and the cathodic peak at $0.8 \mathrm{~V}$ corresponds to the reduction of the surface Au oxide. The integrated charge of this reduction peak (indicated by the *) serves as a measure of the electrochemical SA of the Au. These two CVs show non-aggregated and aggregated samples with a similar SA. Frame B of Figure 4.1 shows the stripping voltammetry of the same two samples (non-aggregated and aggregated $4 \mathrm{~nm}$ Au NPs) obtained by sweeping the potential from $-0.2 \mathrm{~V}$ to $1.6 \mathrm{~V}$ in a solution of $0.1 \mathrm{M} \mathrm{HClO} 4$ plus $0.01 \mathrm{M} \mathrm{KBr}$. The anodic peak near $0.8 \mathrm{~V}$ 
corresponds to the stripping (oxidation) of all of the Au on the surface, resulting in dissolved $\mathrm{AuBr}_{4}^{-}$mainly

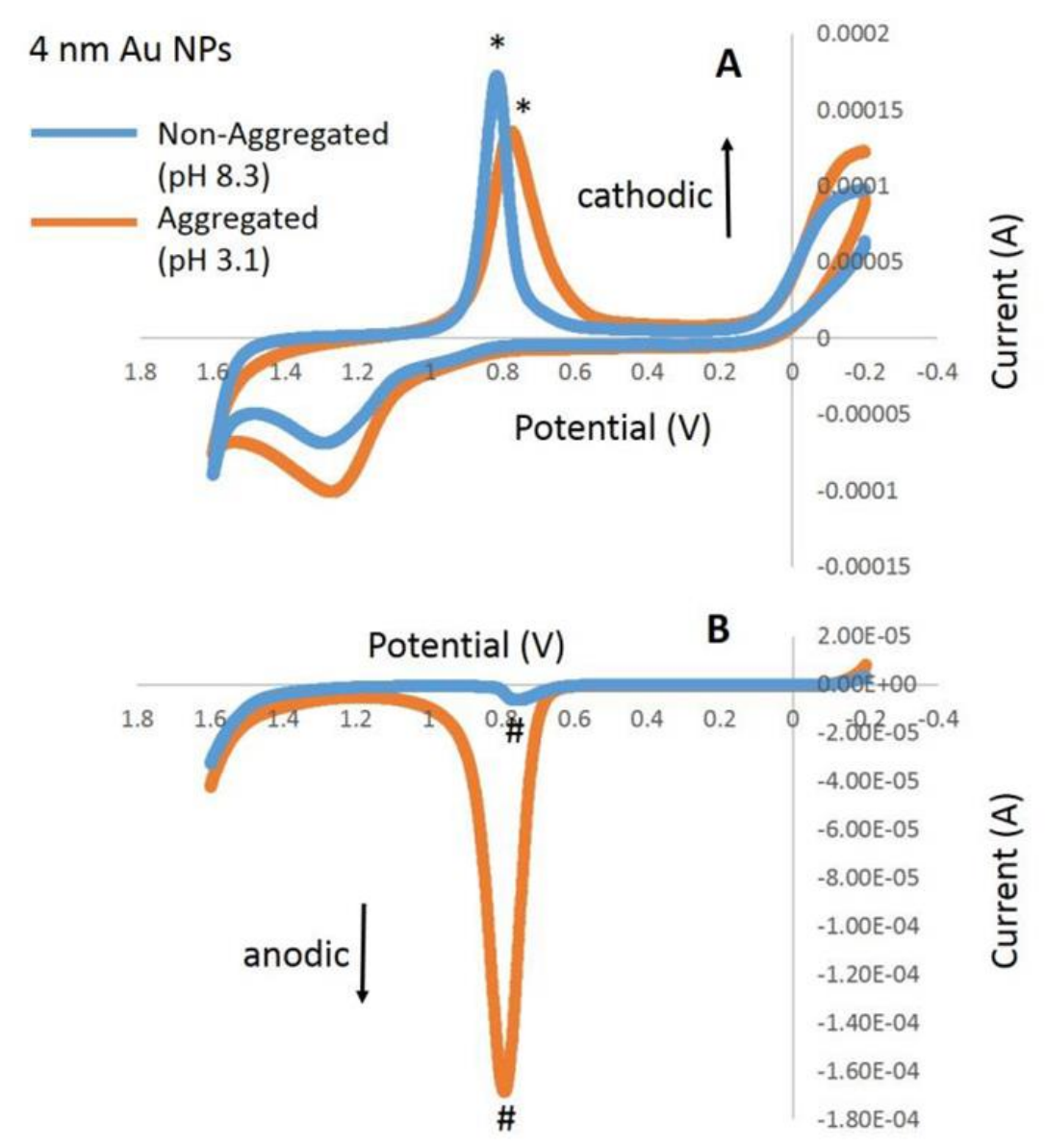

Figure 4. 1. Cyclic voltammograms (A) and linear sweep stripping voltammograms (B) of non-aggregated (blue plots) and aggregated (orange plots) $4 \mathrm{~nm}$ diameter Au NPs attached to glass/ITO/APTES electrodes. The area under the peak marked with a * in the cyclic voltammograms is proportional to the SA of the Au NPs while the area under the peak marked with a \# in the linear sweep stripping voltammogram is proportional to the volume of the Au NPs. Clearly the SA-to-volume ratio is significantly smaller for the aggregated $4 \mathrm{~nm}$ diameter $\mathrm{Au}$ NPs. 
and possibly some $\mathrm{AuBr}_{2}$ - also. Since the integrated charge of this peak (indicated by the \#) corresponds to all of the Au on the electrode, it is proportional to the volume of the Au NPs. It is very clear from Frame $A$ and $B$ that the non-aggregated and aggregated Au NPs had a similar SA, but that the aggregated Au NPs had a much larger volume. This indicates that the SA-to-volume ratio was much bigger for the non-aggregated $4 \mathrm{~nm}$ diameter Au NPs compared to the aggregated ones. Figure 4.2 shows the same data as in Figure 4.1, but for $15 \mathrm{~nm}$ diameter Au NPs attached to glass/ITO/APTES at pH 5 (non-aggregated) and pH 2.8 (aggregated).

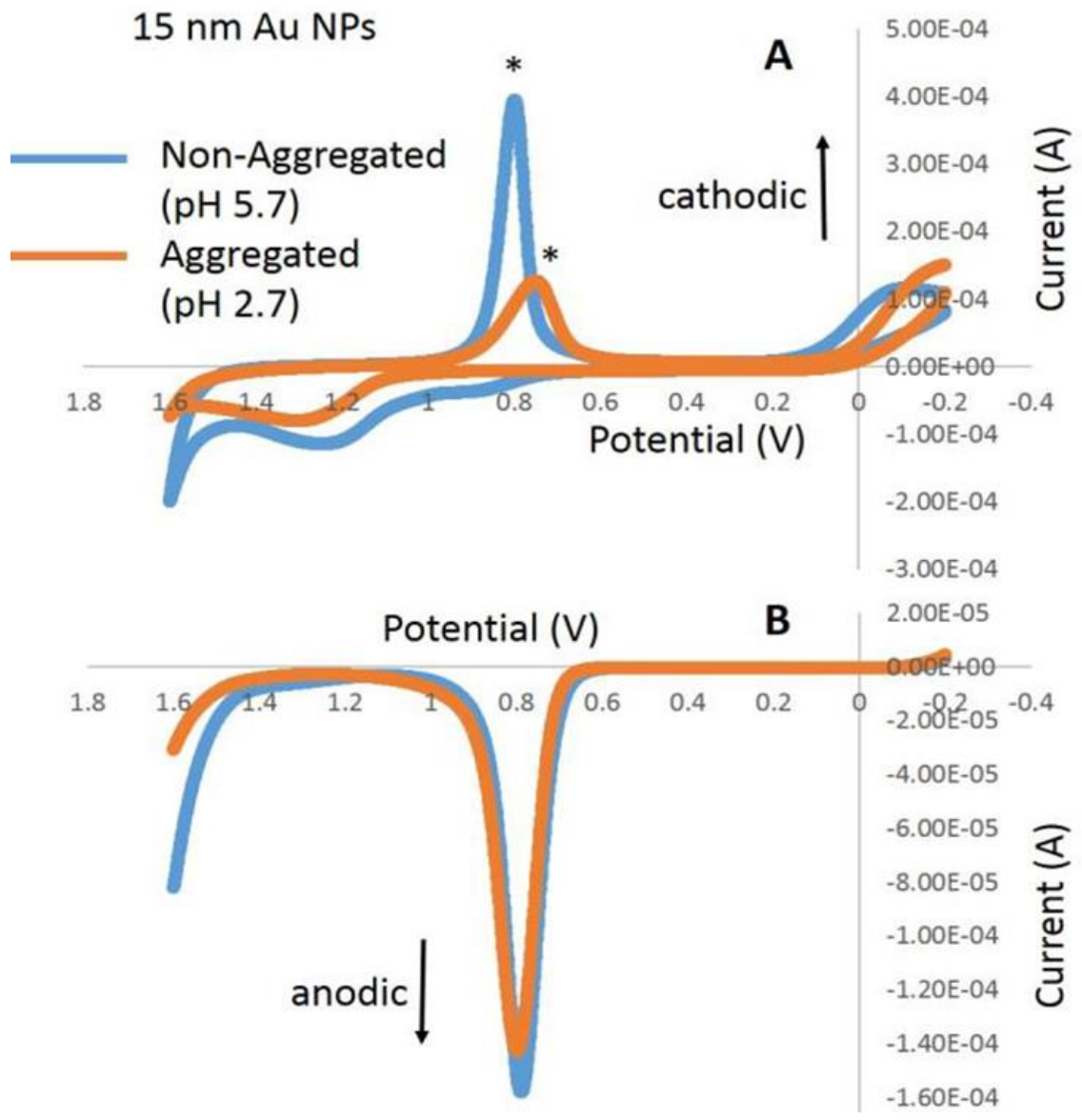


Figure 4. 2. Cyclic voltammograms (A) and linear sweep stripping voltammograms (B) of non-aggregated (blue plots) and aggregated (orange plots) $15 \mathrm{~nm}$ diameter Au NPs attached to glass/ITO/APTES electrodes. The area under the peak marked with a * in the cyclic voltammograms is proportional to the SA of the Au NPs while the area under the peak marked with a \# in the linear sweep stripping voltammogram is proportional to the volume of the Au NPs. Clearly the SA-to-volume ratio is significantly smaller for the aggregated $15 \mathrm{~nm}$ diameter $\mathrm{Au}$ NPs.

For these set of data, it is clear that the non-aggregated $15 \mathrm{~nm}$ diameter $\mathrm{Au}$ NPs have a larger SA, as indicated by the larger cathodic peak at $0.8 \mathrm{~V}$ in Frame A, but very similar volume, as indicated by the similar sized anodic peaks at $0.8 \mathrm{~V}$ in Frame B. This leads to an overall higher SA-to-volume ratio for the nonaggregated Au NPs compared to the aggregated ones. Table 4.1 shows the integrated charge of the cathodic peak at $0.8 \mathrm{~V}$, the integrated charge of the anodic peak at $0.8 \mathrm{~V}$, and the ratio of the two for $4 \mathrm{~nm}$ diameter and $15 \mathrm{~nm}$ diameter $\mathrm{Au}$ NPs that were non-aggregated and aggregated. The average SA-to-volume ratio was $0.32 \pm 0.03$ for the non-aggregated $4 \mathrm{~nm}$ diameter Au NPs and $0.044 \pm 0.005$ for the aggregated ones, which is about a factor of 7 lower. The SA-to-volume ratio was $0.097 \pm 0.006$ for the $15 \mathrm{~nm}$ diameter non-aggregated Au NPs and $0.049 \pm$ 0.007 for the aggregated ones, which is about a factor of 2 lower. These results show that there is a significant reduction in the SA- to-volume ratio upon aggregation of these Au NPs and that it is likely 
Table 4. 1. Electrochemical SA-to-volume ratio measurements for non-aggregated and aggregated $4 \mathrm{~nm}$ diameter and $15 \mathrm{~nm}$ diameter Au NPs.

\begin{tabular}{|c|c|c|c|}
\hline $\begin{array}{c}4 \text { nm diameter Au } \\
\text { NPs "non- } \\
\text { aggregated" }\end{array}$ & $\begin{array}{c}\text { Charge under } \\
\text { cathodic peak in CV } \\
\text { (Coulombs) } \\
\text { "SA" }\end{array}$ & $\begin{array}{c}\text { Charge under } \\
\text { anodic } \\
\text { peak in LSV } \\
\text { (Coulombs) } \\
\text { "volume" }\end{array}$ & $\begin{array}{c}\text { Electrochemical } \\
\text { SA-To- } \\
\text { Volume Ratio }\end{array}$ \\
\hline Sample 1 & $9.96 \times 10^{-5}$ & $3.20 \times 10^{-4}$ & 0.311 \\
\hline Sample 2 & $8.09 \times 10^{-5}$ & $2.76 \times 10^{-4}$ & 0.293 \\
\hline Sample 3 & $12.3 \times 10^{-5}$ & $3.56 \times 10^{-4}$ & 0.346 \\
\hline & & Average \pm std. dev & $0.32 \pm 0.03$ \\
\hline \multicolumn{4}{|l|}{$\begin{array}{l}4 \text { nm diameter Au } \\
\text { NPs "aggregated" }\end{array}$} \\
\hline Sample 1 & $7.59 \times 10^{-5}$ & $1.75 \times 10^{-3}$ & 0.0434 \\
\hline Sample 2 & $7.52 \times 10^{-5}$ & $1.94 \times 10^{-3}$ & 0.0388 \\
\hline Sample 3 & $0.438 \times 10^{-5}$ & $0.0886 \times 10^{-3}$ & 0.0494 \\
\hline & & Average \pm std. dev & $0.044 \pm 0.005$ \\
\hline \multicolumn{4}{|l|}{$\begin{array}{c}15 \text { nm diameter Au } \\
\text { NPs "non- } \\
\text { aggregated" }\end{array}$} \\
\hline Sample 1 & $1.96 \times 10^{-4}$ & $2.02 \times 10^{-3}$ & 0.0970 \\
\hline Sample 2 & $1.66 \times 10^{-4}$ & $1.81 \times 10^{-3}$ & 0.0917 \\
\hline Sample 3 & $1.69 \times 10^{-4}$ & $1.64 \times 10^{-3}$ & 0.1030 \\
\hline & & Average \pm std. dev & $0.097 \pm 0.006$ \\
\hline \multicolumn{4}{|l|}{$\begin{array}{l}15 \mathrm{~nm} \text { diameter Au } \\
\text { NPs "aggregated" }\end{array}$} \\
\hline Sample 1 & $1.43 \times 10^{-4}$ & $2.80 \times 10^{-3}$ & 0.0511 \\
\hline
\end{tabular}




\begin{tabular}{|c|c|c|c|} 
Sample 2 & $0.213 \times 10^{-4}$ & $0.523 \times 10^{-3}$ & 0.0407 \\
\hline Sample 3 & $0.813 \times 10^{-4}$ & $1.48 \times 10^{-3}$ & 0.0549 \\
\hline \multicolumn{2}{|l}{} & Average \pm std. dev. & $0.049 \pm 0.007$ \\
\hline
\end{tabular}

responsible for the observed positive shift in the oxidation potential. While not conclusive, the shift in oxidation potential is consistent with the measured changes in the SA-to-volume ratio. A decrease in SA-to-volume ratio by a factor of 7 is equivalent to an increase in the diameter by a factor of 7 , which would mean the 4 $\mathrm{nm}$ diameter Au NPs would behave like $28 \mathrm{~nm}$ diameter Au NPs on average. The $15 \mathrm{~nm}$ diameter Au NPs would behave like $30 \mathrm{~nm}$ diameter Au NPs on average after aggregation by the same analogy. These are getting closer to bulk like NPs with a higher oxidation potential.

\subsection{Conclusions}

Electrochemical SA/V was found to sensitive to the aggregation in $4 \mathrm{~nm}$ and $15 \mathrm{~nm}$ Au NSs. The SA/V of aggregated Au NSs (4 nm and $15 \mathrm{~nm}$ ) was found to be significantly lower than their non-aggregated forms. The shift in oxidation potential $\left(E_{p}\right)$ of aggregated $4 \mathrm{~nm}$ and $15 \mathrm{~nm}$ Au NSs with change in SA/V from non-aggregated to aggregated form also indicated dependence of $E_{p}$ of Au NSs on their SA/V. 


\section{CHAPTER V}

THE IMPACT OF ASSEMBLY METHOD ON THE SURFACE AREA-TOVOLUME RATIO AND ELECTROCHEMICAL OXIDATION POTENTIAL OF METAL NPS

\subsection{Introduction}

Metal NPs (NPs), such as nanospheres (NSs), have interesting size-dependent optical, electronic, chemical, and electrochemical properties which are useful for a wide variety of applications in catalysis, ${ }^{169-170}$ sensing, ${ }^{118,}$, 171-172 photovoltaics, ${ }^{173}$ nanoelectronics, ${ }^{119}$ imaging, ${ }^{120}$ therapeutics, ${ }^{121}$ and plasmonics. ${ }^{122}$ For these different applications the size plays an important role in the function. In general, smaller-sized NSs with higher SA-to-volume ratio (SA/V) compared to larger NSs and bulk analogues show higher reactivity in catalytic and sensing applications. For example, Back and coworkers theoretically proved that the existence of more corner sites in smaller-sized Au NSs makes them more active for $\mathrm{CO}_{2}$ reduction due to the fact that $\mathrm{CO}_{2}$ reduction is highly favored at these corner sites. ${ }^{15}$ Later, Mistry and coworkers also observed experimentally that the $\mathrm{CO}_{2}$ reduction reactivity increased with a decrease in the size of Au NSs from 8 to $1 \mathrm{~nm}$ due to an increase in the number of corner sites. ${ }^{174}$ As the size of the NS decreases, the SA-to-volume ratio (SA/V) increases, which leads to unique surface chemical and electrochemical properties. Despite their useful properties, the small size and large SA/V leads to stability issues as they are prone to oxidize at lower potentials ${ }^{175-177}$ and are relatively less stable against ripening (size increase) $)^{178}$ due to the driving force to reduce their surface free energy by reducing the SA/V. 
Ripening and aggregation processes reduce SA/V and also usually removes the desirable electrochemical and surface chemical properties of the nanostructures. Early work by Henglein ${ }^{147}$ and Plieth ${ }^{148}$ described the decreasing oxidation potential of metal NSs with decreasing size. Henglein calculated the negative shift in oxidation potential of metal NSs with decreasing size based on their sublimation energies while Plieth predicted a negative shift relative to their bulk values based on differences in Gibbs surface free energies, proportional to SA.

Later, different experimental approaches showed the size-dependent oxidation of metal NSs. For example, Sieradzki and co-workers used Electrochemical Scanning Tunneling Microscopy (ECSTM) to show that Pt NPs $<4$ $\mathrm{nm}$ in diameter dissolved at potentials well negative of their bulk potential value and they oxidized directly by forming soluble $\mathrm{Pt}^{2+}$ cations, while the bulk Pt oxidized by forming $\mathrm{Pt}$ oxide. ${ }^{149}$ Brus and co-workers used SEM to show the electrochemical Ostwald ripening of Ag NPs (NPs) on glass/ITO, which occurred due to the different standard potentials between the small-sized and larger-sized Ag NPs. ${ }^{179}$ Ivanova and Zamborini observed a size-dependent shift in $\mathrm{E}_{\mathrm{p}, \mathrm{ox}}$ for $\mathrm{Ag}$ NSs ${ }^{85}$ of $8-50 \mathrm{~nm}$ diameter in acidic solution and for Au NSs ${ }^{176}$ in acidic bromide using anodic stripping voltammetry (ASV). Brainina and co-workers also observed experimentally (and theoretically) a shift in $E_{p, o x}$ for Au and Ag NSs using ASV. ${ }^{152,}$ 175, 180 In addition, ASV has been used to determine the composition and atomic arrangement of bimetallic NSs. ${ }^{181-182}$

Surfaces with high curvature shows an oxidation potential more negative with respect to a surface having low curvature. ${ }^{183}$ Likewise, with regard to NSs and 
their surface features, those with a smaller radius of curvature are energetically unfavorable and more easily prone to oxidative dissolution. ${ }^{184}$ Regarding nanostructure shape, Hwang and co-workers observed that $\mathrm{Ag}$ nanostructures exhibited oxidation potentials in the order of $\mathrm{Ag}$ nanocubes (43 nm edge, $346 \mathrm{mV}$ ) $>$ nanospheres $(53 \mathrm{~nm}$ diameter, $337 \mathrm{mV})>$ pentatwin decahedrons $(86 \mathrm{~nm}$ in edge length, $315 \mathrm{mV}$ ) > triangular nanoplates $(127 \mathrm{~nm}$ in edge length and $11 \mathrm{~nm}$ in thickness, $293 \mathrm{mV}) .{ }^{185}$ They attributed the change in $E_{p, o x}$ for the different shapes partially to differences in SA/V but also to differences in the surface energy of atoms on the different crystalline facets, since the calculated SA/V of the cubes (0.140) was larger than the SA/V of the nanospheres (0.113), but the $E_{p, o x}$ (cube) was more positive. In addition to size and shape, the oxidation potential of metal NSs is also related to others factors, such as the type of ligand used as a stabilizer during synthesis, ${ }^{186}$ electrode material, ${ }^{155}$ and electrolyte. ${ }^{177}$ These factors will also play a significant role in the reactivity relevant to catalysis and sensing. In terms of electrode materials, Brainina and coworkers observed a positive shift in $E_{p, o x}$ arising from interactions of NSs with an electrode surface having a work function more positive of that of the NS. ${ }^{82}$ This acts as a stabilizing factor that can counteract the destabilizing effect of the small NS size (high curvature and high $S A / V)$. Accordingly, they demonstrated a positive shift in $E_{p, o x}$ for $10 \mathrm{~nm}$ diameter Au NSs on a Pt disk electrode compared to that on a glassy carbon disk electrode, but no significant shift for $150 \mathrm{~nm}$ diameter Au NSs on the two substrates. The larger influence of the electrode material on the smaller Au NSs was attributed to more points of interaction between the NSs and the electrode. 
Masitas et al. later also studied the effect of electrode material on the sizedependent oxidation of $\mathrm{Au}$ and Ag NSs. ${ }^{155}$ They observed that the $\mathrm{E}_{\mathrm{p}, \mathrm{ox}}$ of $9 \mathrm{~nm}$ diameter citrate-capped Ag NSs decreased appreciably as the electrode material was changed from an Au or Pt disc electrode to glassy carbon and glass/ITO. Au NSs greater than $10 \mathrm{~nm}$ in diameter showed different $E_{p, o x}$ values when directly attached to glass/ITO as compared to when attached to glass/ITO through an amine-terminated silane linker, which they attributed to the interfacial potential created by the positively charged, protonated amine groups. These studies demonstrate the differences in electrochemical reactivity of metal NSs attached to different electrode materials in different ways, but the reasons are not very well understood. Masitas and Allen recently reported the size-dependent electrophoretic deposition (EPD) of citrate-stabilized Au NPs. ${ }^{187}$ EPD is a commonly used method for NS assembly on electrode surfaces for different applications. ${ }^{188-189}$ During the course of those studies, they noticed higher $E_{p, 0 x}$ values for NSs assembled by EPD versus chemical methods of assembly (aminefunctionalized silane linker) for the same size Au NSs. Allen et al. showed an even more dramatic positive shift in $E_{p, o x}$ for the same-sized NSs that were aggregated relative to those that were not aggregated, which was attributed to a decrease in SA/V upon aggregation. ${ }^{88}$ The electrochemical method used for measuring SA/V also provided a direct measure of the average diameter for surface-attached NSs. ${ }^{156}$ Our previous SA/V studies along with the observation of different $E_{p, o x}$ values for Au NSs assembled in different ways motivated this work, leading to the current hypothesis that different assembly methods lead to different SA/V for the 
same size Au NSs, which results in a different $E_{p, o x}$. In this work we directly measure the $E_{p, o x}$ and $S A / V$ for different sized Au NSs assembled by different assembly methods to test this hypothesis.

\subsection{Experimental Section}

The chemicals, optical characterization, microscopic characterization and electrochemical SA/V assessment are described in the experimental procedures in Chapter II.

\subsubsection{Synthesis of $1.6 \mathrm{~nm}$ Average Diameter THPC Stabilized Au} Nanospheres (NSs). We synthesized $1.6 \mathrm{~nm}$ diameter THPC-stabilized Au NPs using the procedure described by Duff and co-workers. ${ }^{109}$ Briefly, $500 \mu \mathrm{L}$ of $0.2 \mathrm{M}$ $\mathrm{NaOH}$ solution was added to glass vials containing $15.16 \mathrm{~mL}$ of nanopure water followed by the addition of $400 \mu \mathrm{L}$ of the reducing agent THPC (200 $\mu \mathrm{L}$ of $80 \%$ THPC diluted to $16.66 \mathrm{~mL}$ of nanopure water). Finally, $660 \mu \mathrm{L}$ of 25 $\mathrm{mM} \mathrm{HAuCl}{ }_{4} \cdot 3 \mathrm{H}_{2} \mathrm{O}$ was added to the vials with constant stirring. After the addition of $\mathrm{HAuCl}_{4} \cdot 3 \mathrm{H}_{2} \mathrm{O}$, immediately an orange-brown color formed in solution, indicative of small Au NSs.

\subsubsection{Drop-cast deposition of $1.6 \mathrm{~nm}, 4 \mathrm{~nm}$ and $15 \mathrm{~nm}$ Au NPs mixed with} different concentrations of carbon black (CB) directly on Glass/ITO. We prepared carbon black (CB) mixed with Au NSs of three different sizes (1.6 nm, 4 $\mathrm{nm}$ and $15 \mathrm{~nm}$ ) based on the work by Crooks and coworkers ${ }^{186}$ and drop-cast deposited them on glass/ITO. For this, glass/ITO electrodes were cleaned by sonication for 20 min each in acetone, ethanol, and 2-propanol before drying under $\mathrm{N}_{2}$. Au NSs of $1.6 \mathrm{~nm}, 4 \mathrm{~nm}$ and $15 \mathrm{~nm}$ sizes were mixed with different amounts 
of $\mathrm{CB}$ and drop-cast deposited onto glass/ITO. The mixtures of Au NSs and CB were prepared by mixing $1 \mathrm{~mL}$ of Au NS solutions with $4 \mathrm{mg}, 1 \mathrm{mg}$, or $0.5 \mathrm{mg}$ of vulcan CB suspended in $400 \mu \mathrm{L}$ of 2 -propanol to prepare the $0.28 \%, 0.071 \%$, and $0.036 \%$ weight(in grams)/volume(in $\mathrm{mL}$ ) solutions, respectively. $5 \mu \mathrm{L}$ of the mixture was drop-cast deposited onto glass/ITO in the case of $4 \mathrm{~nm}$ and $15 \mathrm{~nm}$ Au NSs, while $2 \mu \mathrm{L}$ of the mixture was used for $1.6 \mathrm{~nm}$ Au NSs.

5.2.3. Ozone treatment of Au NS samples. Ozone treatment of samples was performed with a Jelight Company Inc. UVO CLEANER model no. 42 for 15 minutes for the samples indicated in Table S1 of the Supporting Information. Ozone was always performed after assembly of the Au NSs onto the electrodes right before electrochemical characterization.

5.2.4. Oxygen reduction reaction (ORR) experiments. $\mathrm{O}_{2}$ reduction experiments were carried out in a closed glass cell containing three electrodes (glass/ITO/APTES/NSs working electrode, Pt wire counter electrode and $\mathrm{Ag} / \mathrm{AgCl}$

reference electrode) in an $\mathrm{O}_{2}$-saturated $0.1 \mathrm{M} \mathrm{HClO}_{4}$ solution. The $\mathrm{CV}$ was scanned from $0.2 \mathrm{~V}$ to $-0.6 \mathrm{~V}$ at a scan rate of $0.01 \mathrm{~V} / \mathrm{s}$ versus an $\mathrm{Ag} / \mathrm{AgCl}$ reference electrode. The same experiments were carried out in $\mathrm{N}_{2}$-saturated $0.1 \mathrm{M} \mathrm{HClO}_{4}$ solution.

\subsection{Results and Discussion}

We synthesized Au NSs ranging from $1.6 \mathrm{~nm}$ to $70 \mathrm{~nm}$ in diameter for this study using citrate stabilizer in all cases except for the $1.6 \mathrm{~nm}$ diameter Au NSs, where we used tetrakis (hydroxymethyl) phosphonium chloride (THPC) stabilizer. We 
analyzed the sizes of the as-synthesized Au NSs by UV-Vis spectroscopy, transmission electron microscopy (TEM) and scanning electron microscopy (SEM) as shown in Figure 5.1. We normalized the UV-Vis spectra of the different Au NSs to an absorbance of 1.0 at the localized surface plasmon resonance (LSPR) peak, except for the $1.6 \mathrm{~nm}$ diameter NSs, where we normalized it at $\square=400 \mathrm{~nm}$. The LSPR peak shifted positive with increasing NS diameter, consistent with their size from $4 \mathrm{~nm}$ diameter to $70 \mathrm{~nm}$ diameter, ${ }^{112}$ while the $1.6 \mathrm{~nm}$ diameter Au NSs sample showed no prominent LSPR peak as expected for this size. ${ }^{178,190}$ We attached $4 \mathrm{~nm}$ to $70 \mathrm{~nm}$ diameter Au NSs to aminopropyltriethoxy silane (APTES)functionalized glass/ITO electrodes and to APTES-functionalized $\mathrm{SiO}_{2}$-coated $\mathrm{Au}$ TEM grids for SEM and TEM measurements, respectively. Figure 5.1. B shows a TEM image of $1.6 \mathrm{~nm}$ Au NSs while Figures 5.1., C-G shows SEM images of 4, 15, 32, 50 and $70 \mathrm{~nm}$ diameter Au NSs. The images confirm all of the NS sizes. 

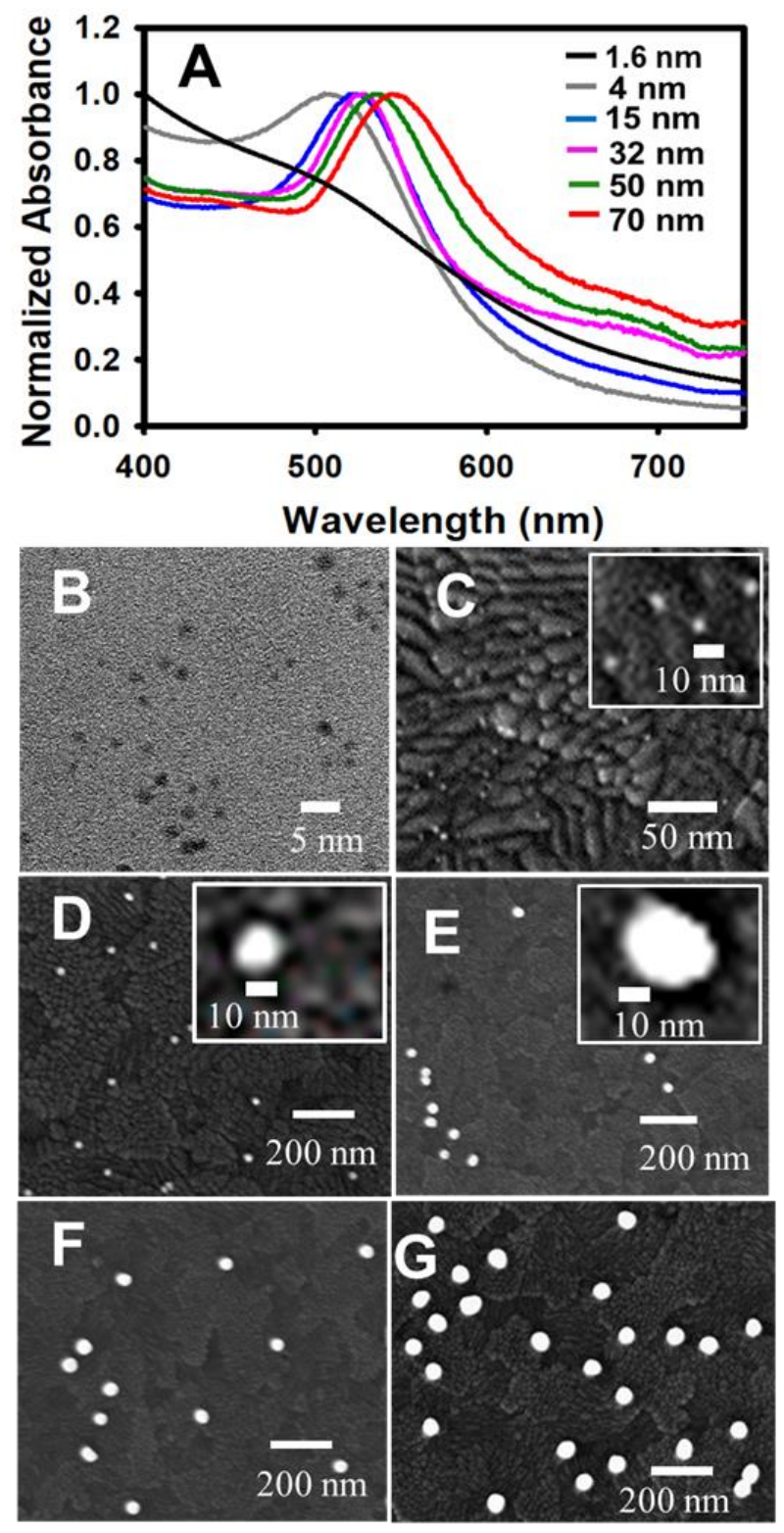

Figure 5. 1. UV-Vis spectra (A) and TEM image (B) of $1.6 \pm 0.4 \mathrm{~nm}$ THPC Au NPs and SEM images (C-G) of $4.1 \pm 0.7,15.1 \pm 1.8,31.2 \pm 1.9,50.1 \pm 3.0$, and $70.8 \pm$ $2.7 \mathrm{~nm}$ of citrate-coated Au nanospheres (NSs).

Ivanova and co-workers previously showed that the peak oxidation potential $\left(E_{p, o x}\right)$ shifts negative for $\mathrm{Au}^{178}$ and $\mathrm{Ag} \mathrm{NSs}{ }^{85}$ with decreasing size in anodic stripping voltammetry (ASV). The magnitude of the shift is generally consistent with Plieth 
theory. ${ }^{148}$ Figure 5.2. shows ASVs of $1.6 \mathrm{~nm}$ (Figure 5.2.A), $4 \mathrm{~nm}$ (Figure 5.2.B) and $15 \mathrm{~nm}$ (Figure 5.2.C) Au NSs attached to a glass/ITO electrode
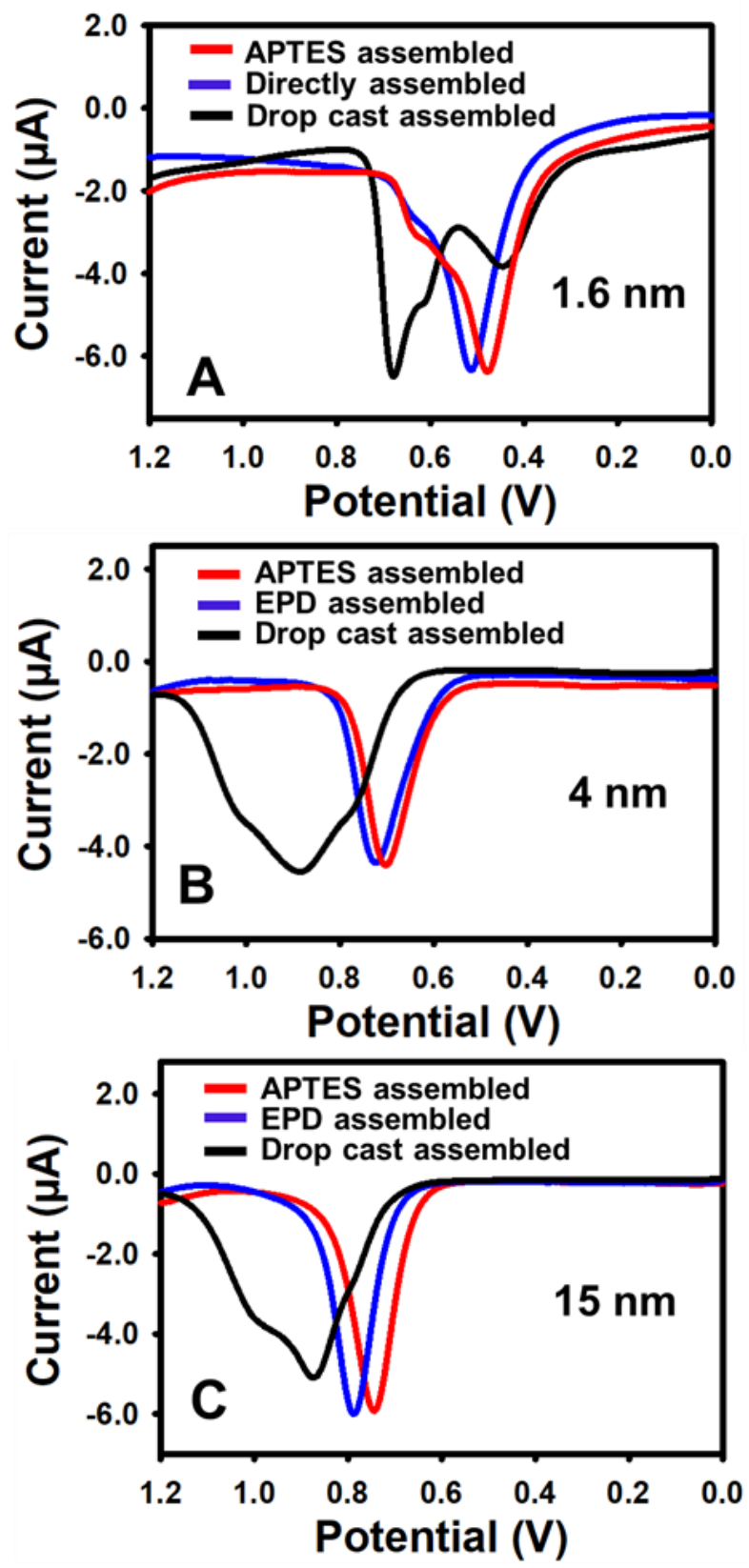

Figure 5. 2. ASVs of $1.6 \mathrm{~nm}(\mathrm{~A}), 4 \mathrm{~nm}(\mathrm{~B})$ and $15 \mathrm{~nm}(\mathrm{C})$ diameter Au NSs assembled onto glass/ITO electrodes through APTES linker (red), by 
electrophoretic deposition (blue), and by drop-cast deposition (black). ASV was performed in $10 \mathrm{mM} \mathrm{KBr}$ plus $0.1 \mathrm{M} \mathrm{KClO}_{4}$ solution with a scan rate of $0.01 \mathrm{~V} / \mathrm{s}$.

electrostatically through an APTES linker, directly to unmodified glass/ITO by electrophoretic deposition (EPD) or direct assembly (soaking), and by drop-cast deposition directly onto unmodified glass/ITO. E $E_{p, o x}$ for Au NSs decreased with decreasing size for NSs assembled by either APTES linkage, EPD, or direct assembly by soaking and rinsing. The $E_{p, o x}$ values are $0.45-0.50 \mathrm{~V}, 0.70-0.75 \mathrm{~V}$, and $0.75-0.80 \mathrm{~V}$ for the $1.6 \mathrm{~nm}, 4 \mathrm{~nm}$, and $15 \mathrm{~nm}$ diameter Au NSs, respectively. We assume that the stabilizer has a negligible passivating effect and that the $E_{p, o x}$ values reflect the size-dependent thermodynamic surface properties of the $\mathrm{Au}$ metal.

In addition to the size dependence, there is a clear dependence on the assembly method. For all three sizes, the Au NSs assembled by EPD ${ }^{191}$ or direct soaking (blue plots) exhibited $\mathrm{E}_{\mathrm{p}, \mathrm{ox}}$ values more positive by $30-50 \mathrm{mV}$ compared to the NSs assembled through the APTES linker (red plots). In the case of the 1.6 nm diameter THPC Au NSs, we could not perform EPD assembly because the charge of THPC is positive. We used direct assembly by soaking in Au NSs, rinsing with water, and drying under $\mathrm{N}_{2}$ to attach the $1.6 \mathrm{~nm}$ diameter THPC Au NSs directly to glass/ITO in order to compare to the NSs attached through the APTES linker. The $\mathrm{E}_{\mathrm{p}, \mathrm{x}}$ for the directly assembled $1.6 \mathrm{~nm}$ diameter THPC Au NSs was found to be shifted positive by about $40 \mathrm{mV}$ as compared to the APTESassembled ones. The $E_{p, o x}$ shifted much more positive for the drop-cast deposited NSs (black plots). The shift was about $+225 \mathrm{mV}$ for the $1.6 \mathrm{~nm}$ Au NSs, +200 
$\mathrm{mV}$ for $4 \mathrm{~nm}$ Au NSs, and $+100 \mathrm{mV}$ for $15 \mathrm{~nm}$ Au NSs. There was a $+20 \mathrm{mV}$ shift in $E_{p, o x}$ for EPD-deposited $4 \mathrm{~nm}$ Au NSs compared to the APTES assembled ones (Figure 1B, blue and red graphs). There was also often several peaks in the ASV. For example, two main $E_{p, o x}$ peaks appeared at $0.45 \mathrm{~V}$ and $0.68 \mathrm{~V}$ for drop-cast deposited $1.6 \mathrm{~nm}$ diameter Au NSs (Figure 5.2.A, black plot). We attribute this to a mixture of well-isolated $1.6 \mathrm{~nm}$ diameter $\mathrm{Au}$ NSs on the surface $\left(E_{p, 0 x} \sim 0.45-0.50\right.$ V) and aggregated $1.6 \mathrm{~nm}$ diameter Au NSs on the surface $\left(E_{p, 0 x} \sim 0.70 \mathrm{~V}\right)$. NSs tend to aggregate during solvent evaporation when assembling by drop-cast deposition.

Our hypothesis in this study was that the positive shifts in $E_{p, o x}$ in Figure 5.2. for EPD, direct assembly, or drop-cast deposition are due to differences in the exposed SA/V for the different NSs assembled in these different ways. To test this, we measured the electroactive SA (SA) and total volume (V) electrochemically as described previously ${ }^{88}$ for the same NSs assembled in these different ways. For SA measurements, cyclic voltammetry $(\mathrm{CV})$ was performed in $0.1 \mathrm{M} \mathrm{HClO}_{4}$ solution by scanning from 0.2 to $1.4 \mathrm{~V} \mathrm{vs}$. $\mathrm{Ag} / \mathrm{AgCl}(3 \mathrm{M} \mathrm{KCl})$. The area of the $\mathrm{Au}_{2} \mathrm{O}_{3}$ reduction peak in the $\mathrm{CV}$ corresponds to the Coulombs of charge for reaction 1 below, which is proportional to the total sum SA of all the Au NSs on the electrode.

$$
\mathrm{Au}_{2} \mathrm{O}_{3}+6 \mathrm{H}^{+}+6 \mathrm{e}^{-} \leftrightharpoons 2 \mathrm{Au}+3 \mathrm{H}_{2} \mathrm{O} \quad \mathrm{SA}(\mathrm{SA})
$$

We then determined the total volume by measuring the Coulombs of charge passed during oxidative stripping of all of the Au NSs on the same electrode in $0.01 \mathrm{M} \mathrm{KBr}$ plus $0.1 \mathrm{M} \mathrm{KClO}_{4}$ according to reaction 2 below. ${ }^{88}$ 


$$
\mathrm{Au}^{0}+4 \mathrm{Br} \longrightarrow \mathrm{AuBr}_{4}^{-}+3 \mathrm{e}^{-} \quad \text { Volume }(\mathrm{V})
$$

Dividing the Coulombs of charge from reaction 1 by the Coulombs of charge from reaction 2 gives the SA/V of the Au NSs assembled on the electrode surface using the different methods.

We observed that the total SA/V of the Au NSs was significantly different for the different assembly methods. Figure 5.3 shows an example of the results for samples of $15 \mathrm{~nm}$ diameter citrate-coated Au NSs with similar volumes of $3.85 \mathrm{x}$ $10^{-5} \mathrm{C}, 4.01 \times 10^{-5} \mathrm{C}$ and $4.20 \times 10^{-5} \mathrm{C}$ for APTES, EPD and the drop-cast deposition assembly methods, respectively (Figure 5.3 B). The SA values were
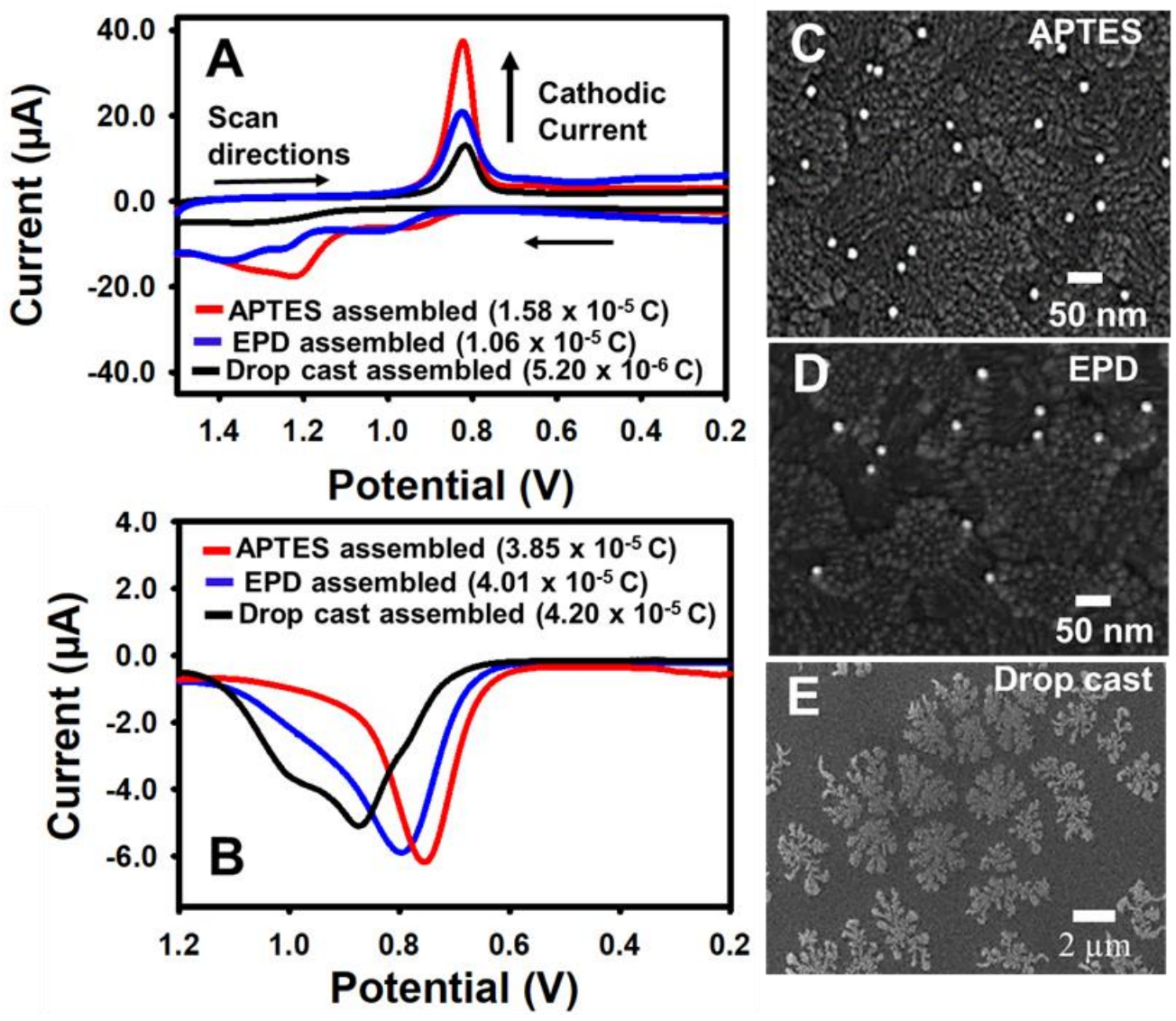
Figure 5. 3. CVs (A) and ASVs (B) and SEM images (C to E) of $15 \mathrm{~nm}$ Au NSs assembled to glass/ITO electrode by different assembly methods. CVs were performed in $0.1 \mathrm{M} \mathrm{HClO}_{4}$ and ASVs performed in $10 \mathrm{mM} \mathrm{KBr}$ plus $0.1 \mathrm{M} \mathrm{KClO}_{4}$ solution with a scan rate of $0.01 \mathrm{~V} / \mathrm{s}$.

$1.58 \times 10^{-5} \mathrm{C}, 1.06 \times 10^{-5} \mathrm{C}$ and $5.20 \times 10^{-6} \mathrm{C}$ for APTES, EPD, and drop-cast deposition, respectively (Figure 5.3, A). After dividing, the measured SA/V for these samples was $0.41,0.26$ and 0.12 for APTES, EPD, and drop-cast deposition, respectively. It appears that the SA/V ratio is inversely proportional to the $E_{p, o x}$ values. This leads us to conclude that the decrease in SA/V is the reason for the positive shift in the $E_{p, o x}$ values for the different assembly methods. Frames C-E of Figure 5.3. show SEM images of the same $15 \mathrm{~nm}$ Au NSs assembled by APTES, EPD, and drop-cast deposition, respectively. There is no obvious visual difference in the images of the NSs assembled by APTES or EPD. In contrast, the drop-cast deposited $15 \mathrm{~nm}$ diameter Au NSs are heavily aggregated compared to APTES or EPD assembly. Table 5.1. provides the electrochemically-measured $S A / V$ and $E_{p, o x}$ values for all of the NS sizes studied and for each assembly method. The table shows the expected trend of increasing $E_{p, o x}$ with decreasing $S A / V$ as a function of increasing NS size for any of the assembly methods. Also, in most cases, the SA/V followed the order from largest to smallest as APTES > EPD or bare soaking $>$ drop-cast deposition, except for the SA/V for EPD and drop-cast deposition for the $70 \mathrm{~nm}$ diameter Au NSs. 
Table 5. 2. SEM/TEM measured radius, average electrochemically-measured $S A / V$, and average $E_{p, o x}$ of Au NSs for different assembly methods. All standard deviations represent one sigma value $(n=3)$.

\begin{tabular}{|c|c|c|c|c|c|c|}
\hline $\begin{array}{l}\text { Au NSs } \\
\text { Radius } \\
\text { (nm) }\end{array}$ & \begin{tabular}{|l|} 
Average \\
SA/V \pm Std. \\
Dev for \\
APTES- \\
attached \\
Au NSs
\end{tabular} & $\begin{array}{l}\text { Average } \\
\text { SA/V } \pm \text { Std. } \\
\text { Dev. for } \\
\text { EPD- } \\
\text { deposited } \\
\text { Au NSs }\end{array}$ & $\begin{array}{l}\text { Average } \\
\text { SA/V } \pm \text { Std. } \\
\text { Dev. for } \\
\text { Drop cast- } \\
\text { deposited } \\
\text { Au NSs }\end{array}$ & $\begin{array}{l}\text { Average } \\
\text { Ep,ox } \pm \text { Std. } \\
\text { Dev. for } \\
\text { APTES- } \\
\text { attached } \\
\text { Au NSs }\end{array}$ & 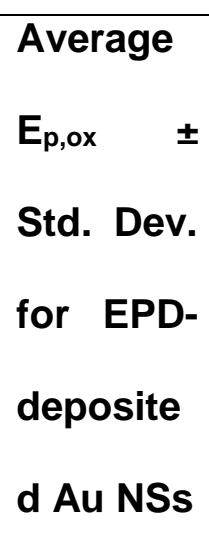 & $\begin{array}{l}\text { Average } \\
E_{p, o x} \pm \text { Std. } \\
\text { Dev. for } \\
\text { Drop cast- } \\
\text { deposited } \\
\text { Au NSs }\end{array}$ \\
\hline $\begin{array}{c}0.80 \pm 0.1 \\
7\end{array}$ & $2.32 \pm .0 .10$ & $1.45 \pm 0.15$ & $\begin{array}{c}0.661 \\
\pm 0.007\end{array}$ & $\begin{array}{c}0.456 \pm \\
0.008\end{array}$ & $\begin{array}{c}0.504 \pm \\
0.015\end{array}$ & $\begin{array}{c}0.769 \\
\pm 0.008\end{array}$ \\
\hline $\begin{array}{c}2.0 \pm 0.01 \\
8\end{array}$ & $\begin{array}{c}0.948 \pm \\
0.019\end{array}$ & $\begin{array}{c}0.801 \pm \\
0.031\end{array}$ & $\begin{array}{c}0.261 \\
\pm 0.036\end{array}$ & $\begin{array}{c}0.714 \pm \\
0.004\end{array}$ & $\begin{array}{c}0.734 \pm \\
0.006\end{array}$ & $\begin{array}{r}0.865 \\
\pm 0.007\end{array}$ \\
\hline $7.5 \pm 0.9$ & $\begin{array}{c}0.370 \pm \\
0.022\end{array}$ & $\begin{array}{c}0.278 \pm \\
0.017\end{array}$ & $\begin{array}{r}0.119 \\
\pm 0.004\end{array}$ & $\begin{array}{c}0.760 \pm \\
0.005\end{array}$ & $\begin{array}{c}0.797 \pm \\
0.008\end{array}$ & $\begin{array}{r}0.852 \\
\pm 0.008\end{array}$ \\
\hline $\begin{array}{c}15.6 \pm \\
0.9\end{array}$ & $\begin{array}{c}0.198 \pm \\
0.020\end{array}$ & $\begin{array}{c}0.080 \pm \\
0.002\end{array}$ & $\begin{array}{r}0.069 \\
\pm 0.007\end{array}$ & $\begin{array}{c}0.815 \pm \\
0.013\end{array}$ & $\begin{array}{c}0.898 \pm \\
0.012\end{array}$ & $\begin{array}{r}0.889 \\
\pm 0.012\end{array}$ \\
\hline $\begin{array}{c}25.1 \pm \\
1.5\end{array}$ & $\begin{array}{c}0.123 \pm \\
0.022\end{array}$ & $\begin{array}{c}0.055 \pm \\
0.004\end{array}$ & $\begin{array}{r}0.045 \\
\pm 0.005\end{array}$ & $\begin{array}{c}0.893 \pm \\
0.011\end{array}$ & $\begin{array}{c}0.944 \pm \\
0.005\end{array}$ & $\begin{array}{r}0.875 \\
\pm 0.013\end{array}$ \\
\hline $\begin{array}{c}35.4 \pm \\
1.4\end{array}$ & $\begin{array}{r}0.079 \\
\pm 0.018\end{array}$ & $\begin{array}{c}0.027 \pm \\
0.003\end{array}$ & $\begin{array}{r}0.039 \\
\pm 0.004\end{array}$ & $\begin{array}{c}0.915 \pm \\
0.013\end{array}$ & $\begin{array}{c}0.982 \pm \\
0.008\end{array}$ & $\begin{array}{r}0.897 \\
\pm 0.021\end{array}$ \\
\hline
\end{tabular}


The $E_{p, o x}$ values also generally showed the reverse trend of APTES $<E P D$ or bare soaking < drop-cast deposition and the $\mathrm{E}_{\mathrm{p}, \mathrm{ox}}$ values for all assembly methods were very close once the bulk size was reached ( $\sim 30 \mathrm{~nm}$ diameter and larger). The best correlation between the $\mathrm{SA} / \mathrm{V}$ and $\mathrm{E}_{\mathrm{p}, \mathrm{ox}}$ values for the different assembly methods occurred for the $15 \mathrm{~nm}$ diameter Au NSs as shown in Figure 5.2.C and 5.3.B (See also Figure 5.4, Supporting Information data for $4 \mathrm{~nm}$ diameter Au NSs for comparison). Table 5.2 provides individual values for electrochemically-measured SA/V and $E_{p, o x}$ values for all of the NS sizes studied and table 5.3 provides oxidation potential values for APTES assembled and drop cast deposited $1.6 \mathrm{~nm}$ Au NSs. 
Table 5. 3. Charge under the oxide reduction peak (CV), charge under the anodic stripping peaks (ASV), SA/V, average SA $/ \mathrm{V}$ ( \pm std. dev.), oxidation peak potentials $\left(E_{p, o x}\right)$, and average oxidation peak potentials ( \pm std. dev.) as a function of NS size and assembly method (with ozone treatment for all sizes except $1.6 \mathrm{~nm}$ Au NSs).

\begin{tabular}{|c|c|c|c|c|c|c|}
\hline $\begin{array}{c}\text { Au NS } \\
\text { assembly } \\
\text { method }\end{array}$ & $\begin{array}{c}\text { CV } \\
\text { Measured } \\
\text { SA } \\
\text { (Coulombs) }\end{array}$ & $\begin{array}{c}\text { ASV } \\
\text { Measured } \\
\text { Volume } \\
\text { (Coulombs) }\end{array}$ & $\begin{array}{c}\text { SA-to- } \\
\text { Volume } \\
\text { Ratio } \\
\text { (SA/V) }\end{array}$ & $\begin{array}{c}\text { Average } \\
\text { SA/V } \pm \text { Std. } \\
\text { Dev. }\end{array}$ & $\begin{array}{c}\text { Oxidation } \\
\text { peak } \\
\text { potential } \\
\left(E_{p, o x}\right)\end{array}$ & $\begin{array}{c}\text { Average } \\
\text { Ep,ox } \pm \\
\text { Std. } \\
\text { Dev. }\end{array}$ \\
\hline \multirow{3}{*}{$\begin{array}{c}4 \mathrm{~nm} \\
\text { APTES } \\
\text { ozone }\end{array}$} & $3.54 \times 10-5$ & $3.76 \times 10-5$ & 0.966 & \multirow[t]{3}{*}{$\begin{array}{c}0.948 \pm \\
0.019\end{array}$} & 0.711 & \multirow[t]{3}{*}{$\begin{array}{c}0.714 \pm \\
0.004\end{array}$} \\
\hline & $5.17 \times 10-5$ & $5.42 \times 10-5$ & 0.953 & & 0.719 & \\
\hline & $3.22 \times 10-5$ & $3.47 \times 10-5$ & 0.927 & & 0.713 & \\
\hline \multirow{3}{*}{$\begin{array}{c}4 \text { nm EPD } \\
\text { ozone }\end{array}$} & $3.40 \times 10-5$ & $4.41 \times 10-5$ & 0.770 & \multirow[t]{3}{*}{$0.801 \pm 0.03$} & 0.728 & \multirow{3}{*}{$\begin{array}{c}0.734 \pm \\
0.006\end{array}$} \\
\hline & $8.50 \times 10-5$ & $1.02 \times 10-4$ & 0.833 & & 0.741 & \\
\hline & $1.48 \times 10-5$ & $1.86 \times 10-5$ & 0.795 & & 0.732 & \\
\hline \multirow{3}{*}{$\begin{array}{c}15 \mathrm{~nm} \\
\text { APTES } \\
\text { ozone }\end{array}$} & $1.27 \times 10-5$ & $3.31 \times 10-5$ & 0.384 & \multirow[t]{3}{*}{$\begin{array}{c}0.370 \pm \\
0.022\end{array}$} & 0.74 & \multirow[t]{3}{*}{$\begin{array}{c}0.747 \pm \\
0.008\end{array}$} \\
\hline & $1.62 \times 10-5$ & $4.23 \times 10-5$ & 0.383 & & 0.757 & \\
\hline & $2.11 \times 10-5$ & $6.12 \times 10-5$ & 0.345 & & 0.746 & \\
\hline \multirow{3}{*}{$\begin{array}{c}15 \mathrm{~nm} \\
\text { EPD } \\
\text { ozone }\end{array}$} & $5.27 \times 10-5$ & $1.90 \times 10-4$ & 0.277 & \multirow[t]{3}{*}{$\begin{array}{c}0.278 \pm \\
0.017\end{array}$} & 0.78 & \multirow[t]{3}{*}{$\begin{array}{c}0.780 \pm \\
0.002\end{array}$} \\
\hline & $1.95 \times 10-5$ & $7.47 \times 10-5$ & 0.261 & & 0.783 & \\
\hline & $1.28 \times 10-5$ & $4.31 \times 10-5$ & 0.296 & & 0.779 & \\
\hline \multirow{2}{*}{$\begin{array}{c}32 \mathrm{~nm} \\
\text { APTES } \\
\text { ozone }\end{array}$} & $4.71 \times 10-6$ & $2.30 \times 10-5$ & 0.204 & \multirow[t]{2}{*}{$\begin{array}{c}0.201 \pm \\
0.002\end{array}$} & 0.795 & \multirow[t]{2}{*}{$\begin{array}{c}0.804 \pm \\
0.009\end{array}$} \\
\hline & $7.96 \times 10-6$ & $3.99 \times 10-5$ & 0.199 & & 0.805 & \\
\hline
\end{tabular}




\begin{tabular}{|c|c|c|c|c|c|c|}
\hline & $1.62 \times 10-5$ & $8.02 \times 10-5$ & 0.201 & & 0.813 & \\
\hline \multirow{3}{*}{$\begin{array}{l}32 \mathrm{~nm} \\
\text { EPD } \\
\text { ozone }\end{array}$} & $9.53 \mathrm{E}-06$ & $1.22 \mathrm{E}-04$ & 0.0781 & \multirow{3}{*}{$\begin{array}{l}0.080 \pm \\
0.002\end{array}$} & 0.897 & \multirow{3}{*}{$\begin{array}{c}0.898 \pm \\
0.012\end{array}$} \\
\hline & $3.34 \times 10-6$ & $4.02 \times 10-5$ & 0.083 & & 0.911 & \\
\hline & $1.48 \times 10-6$ & $1.87 \times 10-5$ & 0.079 & & 0.887 & \\
\hline \multirow{3}{*}{$\begin{array}{l}50 \mathrm{~nm} \\
\text { APTES } \\
\text { ozone }\end{array}$} & $4.26 \times 10-6$ & $2.74 \times 10-5$ & 0.155 & \multirow{3}{*}{$\begin{array}{c}0.123 \pm \\
0.027\end{array}$} & 0.898 & \multirow{3}{*}{$\begin{array}{c}0.904 \pm \\
0.006\end{array}$} \\
\hline & $2.46 \times 10-6$ & $2.15 \times 10-5$ & 0.114 & & 0.906 & \\
\hline & $1.52 \times 10-5$ & $1.49 \times 10-4$ & 0.102 & & 0.910 & \\
\hline \multirow{3}{*}{$\begin{array}{c}50 \mathrm{~nm} \\
\text { EPD } \\
\text { ozone }\end{array}$} & $8.78 \times 10-7$ & $1.71 \times 10-5$ & 0.051 & \multirow{3}{*}{$\begin{array}{c}0.055 \pm \\
0.004\end{array}$} & 0.94 & \multirow[t]{3}{*}{$\begin{array}{l}0.944 \pm \\
0.005\end{array}$} \\
\hline & $3.72 \times 10-6$ & $6.31 \times 10-5$ & 0.059 & & 0.942 & \\
\hline & $6.10 \times 10-6$ & $1.10 \times 10-4$ & 0.055 & & 0.951 & \\
\hline \multirow{3}{*}{$\begin{array}{l}70 \mathrm{~nm} \\
\text { APTES } \\
\text { ozone }\end{array}$} & $4.41 \times 10-5$ & $8.19 \times 10-4$ & 0.054 & \multirow{3}{*}{$\begin{array}{c}0.073 \pm \\
0.017\end{array}$} & 0.911 & \multirow[t]{3}{*}{$\begin{array}{c}0.916 \pm \\
0.006\end{array}$} \\
\hline & $3.73 \times 10-6$ & $4.27 \times 10-5$ & 0.087 & & 0.915 & \\
\hline & $6.31 \times 10-5$ & $8.10 \times 10-4$ & 0.078 & & 0.923 & \\
\hline \multirow{3}{*}{$\begin{array}{l}70 \mathrm{~nm} \\
\text { EPD } \\
\text { ozone }\end{array}$} & $3.69 \times 10-6$ & $1.51 \times 10-4$ & 0.0244 & \multirow[t]{3}{*}{$\begin{array}{c}0.027 \pm \\
0.003\end{array}$} & 0.977 & \multirow{3}{*}{$\begin{array}{c}0.982 \pm \\
0.008\end{array}$} \\
\hline & $2.28 \times 10-6$ & $8.50 \times 10-5$ & 0.0268 & & 0.993 & \\
\hline & $2.32 \times 10-6$ & $7.89 \times 10-5$ & 0.0294 & & 0.978 & \\
\hline \multirow{3}{*}{$\begin{array}{l}1.6 \mathrm{~nm} \\
\text { APTES }\end{array}$} & $5.63 \times 10-4$ & $2.45 \times 10-4$ & 2.30 & \multirow[t]{3}{*}{$2.32 \pm 0.11$} & 0.721 & \multirow{3}{*}{ - } \\
\hline & $1.98 \times 10-4$ & $8.11 \times 10-5$ & 2.44 & & 0.684 & \\
\hline & $5.94 \times 10-6$ & $2.67 \times 10-6$ & 2.22 & & 0.668 & \\
\hline \multirow{2}{*}{$\begin{array}{c}1.6 \mathrm{~nm} \\
\text { Direct } \\
\text { attachment }\end{array}$} & $7.84 \times 10-6$ & $5.51 \times 10-6$ & 1.42 & \multirow[t]{2}{*}{$1.47 \pm 0.15$} & 0.658 & \multirow[b]{2}{*}{-} \\
\hline & $1.50 \times 10-4$ & $9.08 \times 10-5$ & 1.65 & & 0.708 & \\
\hline
\end{tabular}




\begin{tabular}{|c|c|c|c|c|c|c|}
\hline & $1.50 \times 10-5$ & $1.10 \times 10-5$ & 1.36 & & 0.662 & \\
\hline \multirow{3}{*}{$\begin{array}{c}1.6 \mathrm{~nm} 5 \\
\mu \mathrm{L} \text { drop } \\
\text { cast no } \\
\text { ozone }\end{array}$} & $5.51 \times 10-5$ & $1.10 \times 10-4$ & 0.551 & \multirow{3}{*}{$\begin{array}{c}0.615 \pm \\
0.055\end{array}$} & 0.779 & \multirow{3}{*}{$\begin{array}{c}0.769 \pm \\
0.008\end{array}$} \\
\hline & $5.91 \times 10-5$ & $9.10 \times 10-5$ & 0.649 & & 0.762 & \\
\hline & $5.55 \times 10-5$ & $8.49 \times 10-5$ & 0.645 & & 0.768 & \\
\hline \multirow{3}{*}{$\begin{array}{c}1.6 \mathrm{~nm} 5 \\
\mu \mathrm{L} \text { drop } \\
\text { cast no } \\
\text { ozone } \\
0.0005 \mathrm{~g} \mathrm{C}\end{array}$} & $6.21 \times 10-6$ & $9.62 \times 10-6$ & 0.671 & \multirow{3}{*}{$\begin{array}{c}0.661 \pm \\
0.090\end{array}$} & 0.772 & \multirow{3}{*}{$\begin{array}{c}0.770 \pm \\
0.016\end{array}$} \\
\hline & $6.12 \times 10-6$ & $8.20 \times 10-6$ & 0.746 & & 0.754 & \\
\hline & $2.89 \times 10-5$ & $5.10 \times 10-5$ & 0.566 & & 0.786 & \\
\hline \multirow{3}{*}{$\begin{array}{l}1.6 \mathrm{~nm} 5 \\
\mu \mathrm{L} \text { drop } \\
\text { cast with } \\
0.001 \mathrm{~g} \mathrm{C} \\
\text { no ozone }\end{array}$} & $2.21 \times 10-5$ & $5.27 \times 10-5$ & 0.419 & \multirow{3}{*}{$\begin{array}{c}0.354 \pm \\
0.058\end{array}$} & 0.791 & \multirow{3}{*}{$\begin{array}{c}0.791 \pm \\
0.002\end{array}$} \\
\hline & $5^{4.89 \times 10-}$ & $1.60 \times 10-4$ & 0.306 & & 0.790 & \\
\hline & $2.74 \times 10-5$ & $8.10 \times 10-5$ & 0.338 & & 0.794 & \\
\hline \multirow{3}{*}{$\begin{array}{c}1.6 \mathrm{~nm} 5 \\
\mu \mathrm{L} \text { drop } \\
\text { cast no } \\
\text { ozone } \\
0.004 \mathrm{~g} \mathrm{C}\end{array}$} & $4.46 \times 10-5$ & $1.98 \times 10-4$ & 0.225 & \multirow{3}{*}{$\begin{array}{c}0.207 \pm \\
0.028\end{array}$} & 0.827 & \multirow{3}{*}{$\begin{array}{c}0.841 \pm \\
0.012\end{array}$} \\
\hline & $6.35 \times 10-5$ & $2.85 \times 10-4$ & 0.222 & & 0.849 & \\
\hline & $7.45 \times 10-5$ & $4.25 \times 10-4$ & 0.175 & & 0.847 & \\
\hline \multirow{3}{*}{$\begin{array}{c}4 \mathrm{~nm} 5 \mu \mathrm{L} \\
\text { drop cast } \\
\text { ozone }\end{array}$} & $1.29 \times 10-5$ & $4.54 \times 10-5$ & 0.284 & \multirow[t]{3}{*}{$\begin{array}{c}0.261 \pm \\
0.036\end{array}$} & 0.858 & \multirow[t]{3}{*}{$\begin{array}{c}0.865 \pm \\
0.007\end{array}$} \\
\hline & $7.88 \times 10-6$ & $3.59 \times 10-5$ & 0.219 & & 0.873 & \\
\hline & $2.29 \times 10-5$ & $8.14 \times 10-5$ & 0.281 & & 0.865 & \\
\hline \multirow{3}{*}{$\begin{array}{c}4 \mathrm{~nm} 5 \mu \mathrm{L} \\
\text { drop cast } \\
\text { ozone } \\
0.0005 \mathrm{~g} \mathrm{C}\end{array}$} & $3.84 \times 10-5$ & $5.41 \mathrm{E}-05$ & 0.709 & \multirow[t]{3}{*}{$\begin{array}{c}0.669 \pm \\
0.006\end{array}$} & 0.758 & \multirow[t]{3}{*}{$\begin{array}{c}0.755 \pm \\
0.005\end{array}$} \\
\hline & $3.15 \times 10-5$ & $5.31 \mathrm{E}-05$ & 0.593 & & 0.759 & \\
\hline & $9.62 \times 10-6$ & 1.36E-05 & 0.707 & & 0.749 & \\
\hline \multirow{2}{*}{$\begin{array}{c}4 \mathrm{~nm} 5 \mu \mathrm{L} \\
\text { drop cast } \\
\text { with } 0.001 \\
\mathrm{~g} \mathrm{C} \text { ozone }\end{array}$} & $2.61 \times 10-5$ & 5.87E-05 & 0.444 & \multirow[t]{2}{*}{$\begin{array}{c}0.474 \pm \\
0.028\end{array}$} & 0.782 & \multirow[t]{2}{*}{$\begin{array}{c}0.785 \pm \\
0.007\end{array}$} \\
\hline & $2.73 \times 10-5$ & 5.71E-05 & 0.478 & & 0.793 & \\
\hline
\end{tabular}




\begin{tabular}{|c|c|c|c|c|c|c|}
\hline & $1.83 \times 10-5$ & 3.66E-05 & 0.500 & & 0.780 & \\
\hline \multirow{3}{*}{$\begin{array}{c}4 \mathrm{~nm} 5 \mu \mathrm{L} \\
\text { drop cast } \\
\text { with ozone } \\
0.004 \mathrm{~g} \mathrm{C}\end{array}$} & $1.88 \times 10-5$ & $7.10 \mathrm{E}-05$ & 0.265 & \multirow[t]{3}{*}{$\begin{array}{l}0.306 \pm \\
0.035\end{array}$} & 0.806 & \multirow[t]{3}{*}{$\begin{array}{c}0.809 \pm \\
0.005\end{array}$} \\
\hline & $1.74 \times 10-5$ & 5.22E-05 & 0.330 & & 0.807 & \\
\hline & $1.91 \times 10-5$ & 5.91E-05 & 0.323 & & 0.816 & \\
\hline \multirow{3}{*}{$\begin{array}{c}15 \mathrm{~nm} 5 \\
\mu \mathrm{L} \text { drop } \\
\text { cast ozone }\end{array}$} & $1.00 \times 10-5$ & 8.69E-05 & 0.115 & \multirow{3}{*}{$\begin{array}{c}0.119 . \pm \\
0.004\end{array}$} & 0.843 & \multirow{3}{*}{$\begin{array}{c}0.852 \pm \\
0.008\end{array}$} \\
\hline & $9.52 \times 10-6$ & 7.72E-05 & 0.123 & & 0.856 & \\
\hline & $9.42 \times 10-6$ & 7.82E-05 & 0.12 & & 0.859 & \\
\hline \multirow{3}{*}{$\begin{array}{c}15 \mathrm{~nm} 5 \\
\mu \mathrm{L} \text { drop } \\
\text { cast ozone } \\
0.0005 \mathrm{~g} \mathrm{C}\end{array}$} & $1.26 \times 10-5$ & $1.10 \mathrm{E}-04$ & 0.124 & \multirow[t]{3}{*}{$0.137 \pm 0.016$} & 0.786 & \multirow[t]{3}{*}{$\begin{array}{c}0.784 \pm \\
0.003\end{array}$} \\
\hline & $7.96 \times 10-6$ & 5.15E-05 & 0.155 & & 0.787 & \\
\hline & $6.08 \times 10-6$ & 4.60E-05 & 0.132 & & 0.780 & \\
\hline \multirow{3}{*}{$\begin{array}{c}15 \mathrm{~nm} 5 \\
\mu \mathrm{L} \text { drop } \\
\text { cast with } \\
0.001 \mathrm{~g} \mathrm{C} \\
\text { ozone }\end{array}$} & $2.43 \times 10-5$ & 2.37E-04 & 0.103 & \multirow{3}{*}{$\begin{array}{c}0.086 \pm \\
0.015\end{array}$} & 0.793 & \multirow{3}{*}{$\begin{array}{c}0.798 \pm \\
0.007\end{array}$} \\
\hline & $5.53 \times 10-6$ & 7.56E-05 & 0.073 & & 0.795 & \\
\hline & $1.97 \times 10-5$ & 2.36E-04 & 0.083 & & 0.806 & \\
\hline \multirow{3}{*}{$\begin{array}{c}15 \mathrm{~nm} 5 \\
\mu \mathrm{L} \text { drop } \\
\text { cast with } \\
\text { ozone } \\
0.004 \mathrm{~g} \mathrm{C}\end{array}$} & $7.36 \times 10-6$ & 1.47 E-04 & 0.050 & \multirow[t]{3}{*}{$0.053 \pm 0.003$} & 0.836 & \multirow{3}{*}{$\begin{array}{c}0.825 \pm \\
0.009\end{array}$} \\
\hline & $1.56 \times 10-5$ & $2.83 \mathrm{E}-04$ & 0.055 & & 0.820 & \\
\hline & $1.62 \times 10-5$ & 2.96E-04 & 0.054 & & 0.819 & \\
\hline \multirow[t]{3}{*}{$\begin{array}{c}32 \mathrm{~nm} \\
\text { drop cast }\end{array}$} & $5.16 \times 10-6$ & 6.80E-05 & 0.075 & \multirow[t]{3}{*}{$\begin{array}{c}0.069 \pm \\
0.007\end{array}$} & 0.898 & \multirow[t]{3}{*}{$\begin{array}{c}0.889 \pm \\
0.016\end{array}$} \\
\hline & $6.49 \times 10-6$ & 1.01E-04 & 0.061 & & 0.900 & \\
\hline & $1.14 \times 10-6$ & 1.59E-05 & 0.072 & & 0.871 & \\
\hline \multirow{3}{*}{$\begin{array}{c}50 \mathrm{~nm} \\
\text { drop cast }\end{array}$} & $5.53 \times 10-6$ & 1.16E-04 & 0.047 & \multirow[t]{3}{*}{$\begin{array}{l}0.045 \pm \\
0.0005\end{array}$} & 0.863 & \multirow[t]{3}{*}{$\begin{array}{c}0.875 \pm \\
0.013\end{array}$} \\
\hline & $7.72 \times 10-7$ & $2.01 \mathrm{E}-05$ & 0.039 & & 0.889 & \\
\hline & $1.93 \times 10-6$ & 3.95E-05 & 0.048 & & 0.872 & \\
\hline
\end{tabular}




\begin{tabular}{|c|c|c|c|c|c|c|}
\hline \multirow[t]{3}{*}{$\begin{array}{c}70 \mathrm{~nm} \\
\text { drop cast }\end{array}$} & $1.30 \times 10-6$ & $3.58 \mathrm{E}-05$ & 0.036 & \multirow[t]{3}{*}{$\begin{array}{c}0.039 \pm \\
0.004\end{array}$} & 0.873 & \multirow[t]{3}{*}{$\begin{array}{c}0.897 \pm \\
0.021\end{array}$} \\
\hline & $5.90 \times 10-6$ & $1.49 \mathrm{E}-04$ & 0.039 & & 0.905 & \\
\hline & $4.76 \times 10-6$ & $1.08 \mathrm{E}-04$ & 0.044 & & 0.914 & \\
\hline
\end{tabular}

Table 5. 4. Charge under the oxide reduction peak (CV), charge under the anodic stripping peak (ASV), peak oxidation potential ( $\left.E_{p, o x}\right)$, and average peak oxidation potentials ( \pm std. dev.) for $1.6 \mathrm{~nm}$ Au NSs assembled by APTES linking and direct soaking in ITO.

\begin{tabular}{|c|c|c|c|}
\hline Assembly method & $\begin{array}{l}\text { ASV volume of } \\
\text { Au NSs }\end{array}$ & $\begin{array}{l}\text { Oxidation peak } \\
\text { potential }\left(E_{p, o x}\right)\end{array}$ & $\begin{array}{c}\text { Average } \\
\mathrm{E}_{\mathrm{p}, \mathrm{ox}} \pm \text { Std } \\
\text { Dev. }\end{array}$ \\
\hline \multirow{3}{*}{$\begin{array}{c}\text { Glass ITO/APTES/1.6 } \\
\text { nm Au NSs }\end{array}$} & $8.79 \times 10^{-5}$ & 0.466 & \multirow{3}{*}{$\begin{array}{l}0.456 \pm \\
0.008\end{array}$} \\
\hline & $5.01 \times 10^{-5}$ & 0.452 & \\
\hline & $2.76 \times 10^{-5}$ & 0.451 & \\
\hline \multirow{3}{*}{$\begin{array}{l}\text { Glass ITO/1.6 nm Au } \\
\text { NSs (Direct attachment) }\end{array}$} & $1.74 \times 10^{-5}$ & 0.512 & \multirow{3}{*}{$\begin{array}{l}0.504 \pm \\
0.015\end{array}$} \\
\hline & $5.42 \times 10^{-5}$ & 0.515 & \\
\hline & $1.07 \times 10^{-5}$ & 0.487 & \\
\hline
\end{tabular}


Au NSs are often mixed with carbon black (CB) as a conductive solid support material for different electrocatalysis applications. For example, Crooks and coworkers drop-cast deposited $20 \mu \mathrm{L}$ mixtures of Au NSs with Vulcan CB onto 6 mm diameter glassy carbon electrodes (GCEs) in order to study the $\mathrm{CO}_{2}$ reduction reaction. ${ }^{186}$ High SA CB is usually mixed with metal NSs due to its unique stability in electrolyte solutions and because it improves electron transfer in metal NPs assembled on electrode surfaces. ${ }^{192-194}$ Hence, it can act as a good catalyst support to prevent the NSs from degrading and to minimize coalescence in them for their intended application. Keeping this in consideration we studied the effect of different concentrations of $\mathrm{CB}$ (in \%w/v g/mL) on the SA/V and $E_{p, o x}$ of Au NSs. We selected the maximum CB concentration to be mixed with Au NSs based on the Crooks study ${ }^{186}$ and also lowered the CB concentration.

The Figure 5.4. box plot shows that the experimentally-measured electroactive $\mathrm{SA} / \mathrm{V}$ decreased as the amount of $\mathrm{CB}$ increased during drop-cast deposition. The SA/V was much smaller than EPD and APTES-attached Au NSs for the different amounts of $\mathrm{CB}$, but generally larger than drop-cast deposition without $\mathrm{CB}$, especially for lower amounts of CB. CB therefore does a good job of separating the Au NSs and increasing their SA/V relative to straight drop-cast deposition, but is worse than assembly by APTES and EPD in terms of NS SA/V ratio. 


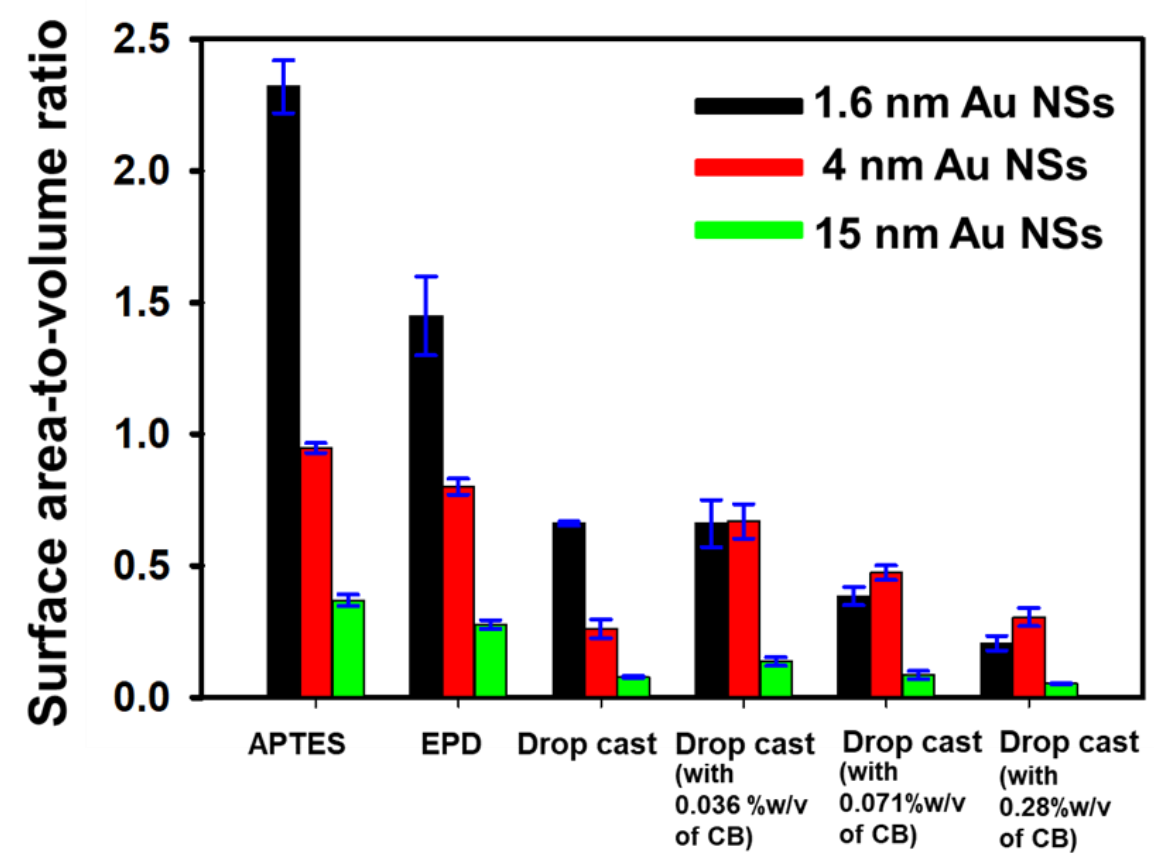

Assembly methods

Figure 5. 4. Box plots comparing the SA/V of 1.6, 4 and $15 \mathrm{~nm}$ Au NSs attached through APTES, EPD ( ${ }^{*}$ except for $1.6 \mathrm{~nm}$ which were attached by direct attachment), drop cast deposition, and drop cast deposition with different concentrations of carbon black (CB) added. Figure 5.5 also shows example data characterizing the different SAs for the different assembly methods for $4 \mathrm{~nm} \mathrm{Au}$ NSs, which had similar ASV coverage. The SA/V decreased with increasing amounts of CB. 
Figure 5.6. below also shows example data characterizing the different SAs for the different assembly methods for $4 \mathrm{~nm}$ Au NSs, which had similar ASV coverage. The SA/V decreased with increasing amounts of $C B$.
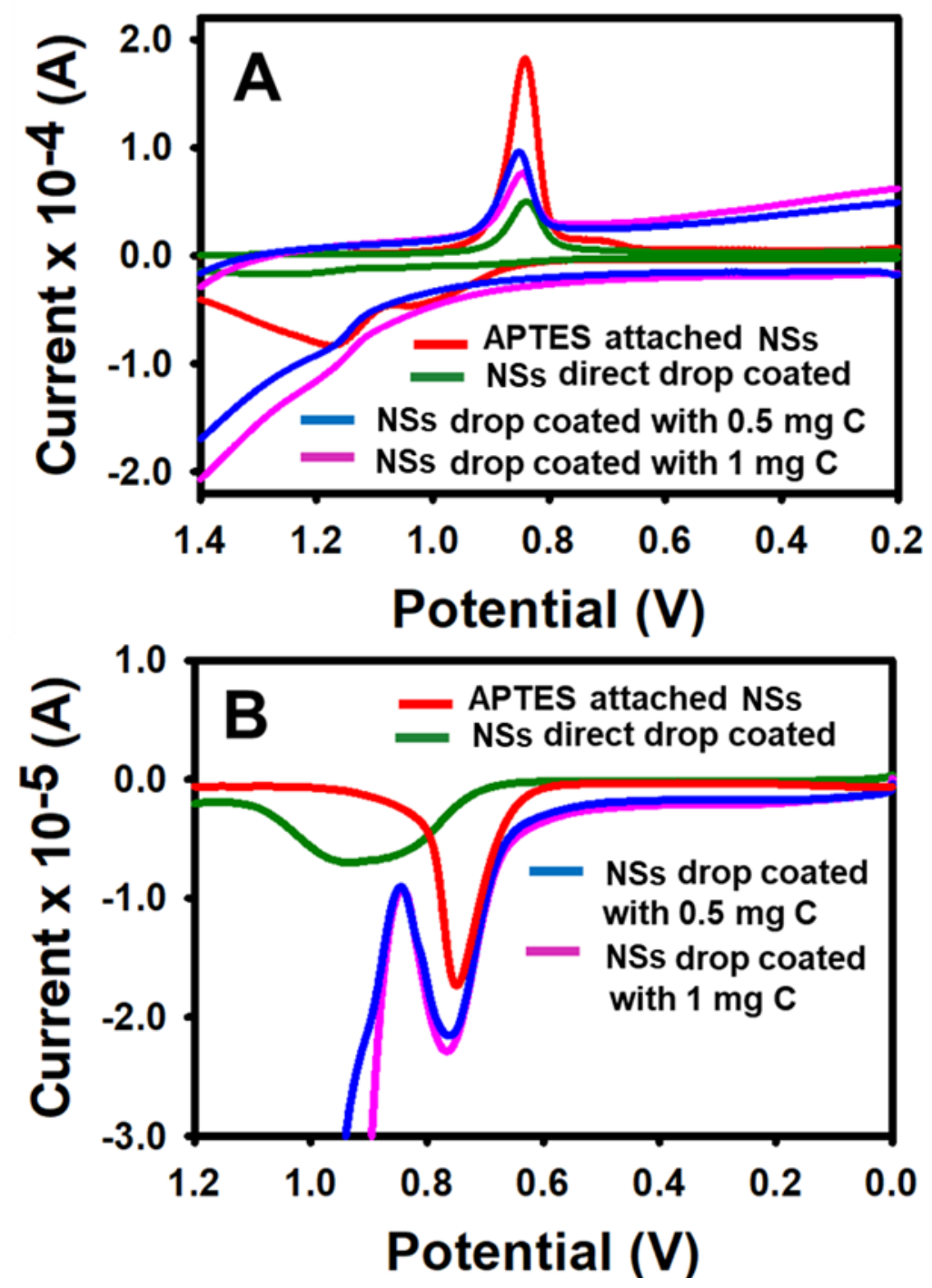

Figure 5. 6. (A) CVs of $4 \mathrm{~nm}$ Au NSs for different assembly methods performed in $0.1 \mathrm{M} \mathrm{HClO}_{4}$ and (B) ASVs performed in $10 \mathrm{mM} \mathrm{KBr}+0.1 \mathrm{M} \mathrm{KClO}_{4}$. 
Accordingly, the $E_{p, o x}$ of the $4 \mathrm{~nm}$ diameter Au NSs with CB shifted positive compared to that for APTES- and EPD-attached $4 \mathrm{~nm}$ Au NSs, consistent with the SA/V being inversely related to the $E_{p, o x}$ (Table 5.4. ).

Table 5. 5. Size of Au NSs, average electrochemically-measured SA/V for drop cast deposited with $0.28 \% \mathrm{w} / \mathrm{v}, 0.072 \% \mathrm{w} / \mathrm{v}$ and $0.036 \% \mathrm{w} / \mathrm{v}$ of C black with Au NSs and average $E_{p, o x}$ for drop cast deposited with $0.28 \% \mathrm{w} / \mathrm{v}, 0.072 \% \mathrm{w} / \mathrm{v}$ and $0.036 \% \mathrm{w} / \mathrm{v}$ of $\mathrm{C}$ black with Au NSs. All standard deviations represent one sigma value $(n=3)$.

\begin{tabular}{|c|c|c|c|c|c|c|}
\hline $\begin{array}{c}\text { Au } \\
\text { NSs } \\
\text { Size } \\
(\mathrm{nm})\end{array}$ & $\begin{array}{c}\text { Average } \\
\text { SA/V } \pm \\
\text { Std. Dev. } \\
\text { for drop } \\
\text { cast } \\
\text { deposited } \\
\text { with } 0.28 \\
\% \text { w/v of C } \\
\text { black with } \\
\text { Au NSs }\end{array}$ & $\begin{array}{c}\text { Average } \\
\text { Ep.ox } \pm \text { Std. } \\
\text { Dev. for } \\
\text { drop cast } \\
\text { deposited } \\
\text { with } 0.28 \\
\% \text { w/v of C } \\
\text { black with } \\
\text { Au NSs }\end{array}$ & $\begin{array}{c}\text { Average } \\
\text { SA/V } \pm \\
\text { Std. Dev. } \\
\text { for drop } \\
\text { cast } \\
\text { deposited } \\
\text { with } 0.072 \\
\% \text { w/v of C } \\
\text { black with } \\
\text { Au NSs }\end{array}$ & $\begin{array}{c}\text { Average } \\
\text { Ep,ox } \pm \text { Std. } \\
\text { Dev. for } \\
\text { drop cast } \\
\text { deposited } \\
\text { with } 0.072 \\
\% \text { w/v of C } \\
\text { black with } \\
\text { Au NSs }\end{array}$ & $\begin{array}{c}\text { Average } \\
\text { SA } / V \pm \\
\text { Std. Dev. } \\
\text { for drop } \\
\text { cast } \\
\text { deposited } \\
\text { with } 0.036 \\
\% \text { w } / v \text { of C } \\
\text { black with } \\
\text { Au NSs }\end{array}$ & $\begin{array}{c}\text { Average } \\
\mathrm{E}_{\mathrm{p}, \mathrm{ox}} \pm \text { Std. } \\
\text { Dev. for } \\
\text { drop cast } \\
\text { deposited } \\
\text { with } 0.036 \\
\% \text { w/v of C } \\
\text { black with } \\
\text { Au NSs }\end{array}$ \\
\hline $\begin{array}{c}1.6 \pm \\
0.4\end{array}$ & $\begin{array}{c}0.207 \pm \\
0.028\end{array}$ & $\begin{array}{c}0.841 \pm \\
0.012\end{array}$ & $\begin{array}{c}0.354 \pm \\
0.058\end{array}$ & $\begin{array}{c}0.791 \pm \\
0.002\end{array}$ & $\begin{array}{c}0.661 \pm \\
0.090\end{array}$ & $\begin{array}{c}0.770 \pm \\
0.016\end{array}$ \\
\hline $\begin{array}{c}4.1 \pm \\
0.7\end{array}$ & $\begin{array}{c}0.306 \pm \\
0.035\end{array}$ & $\begin{array}{c}0.809 \pm \\
0.005\end{array}$ & $\begin{array}{c}0.474 \pm \\
0.028\end{array}$ & $\begin{array}{c}0.785 \pm \\
0.007\end{array}$ & $\begin{array}{c}0.669 \pm \\
0.066\end{array}$ & $\begin{array}{c}0.755 \pm \\
0.005\end{array}$ \\
\hline $\begin{array}{r}15.1 \\
\pm 1.8\end{array}$ & $\begin{array}{c}0.053 \pm 0.00 \\
3\end{array}$ & $\begin{array}{c}0.825 \pm \\
0.009\end{array}$ & $\begin{array}{c}0.086 \pm \\
0.015\end{array}$ & $\begin{array}{c}0.798 \pm \\
0.007\end{array}$ & $\begin{array}{c}0.137 \pm 0.01 \\
6\end{array}$ & $\begin{array}{c}0.784 \pm \\
0.003\end{array}$ \\
\hline
\end{tabular}


To further test our hypothesis that the $E_{p, o x}$ is determined by the measured SA/V of each NS sample, we plotted the $E_{p, o x}$ as a function of SA/V for all of the different assembly methods and different sizes of Au NSs (Figure 5.7).

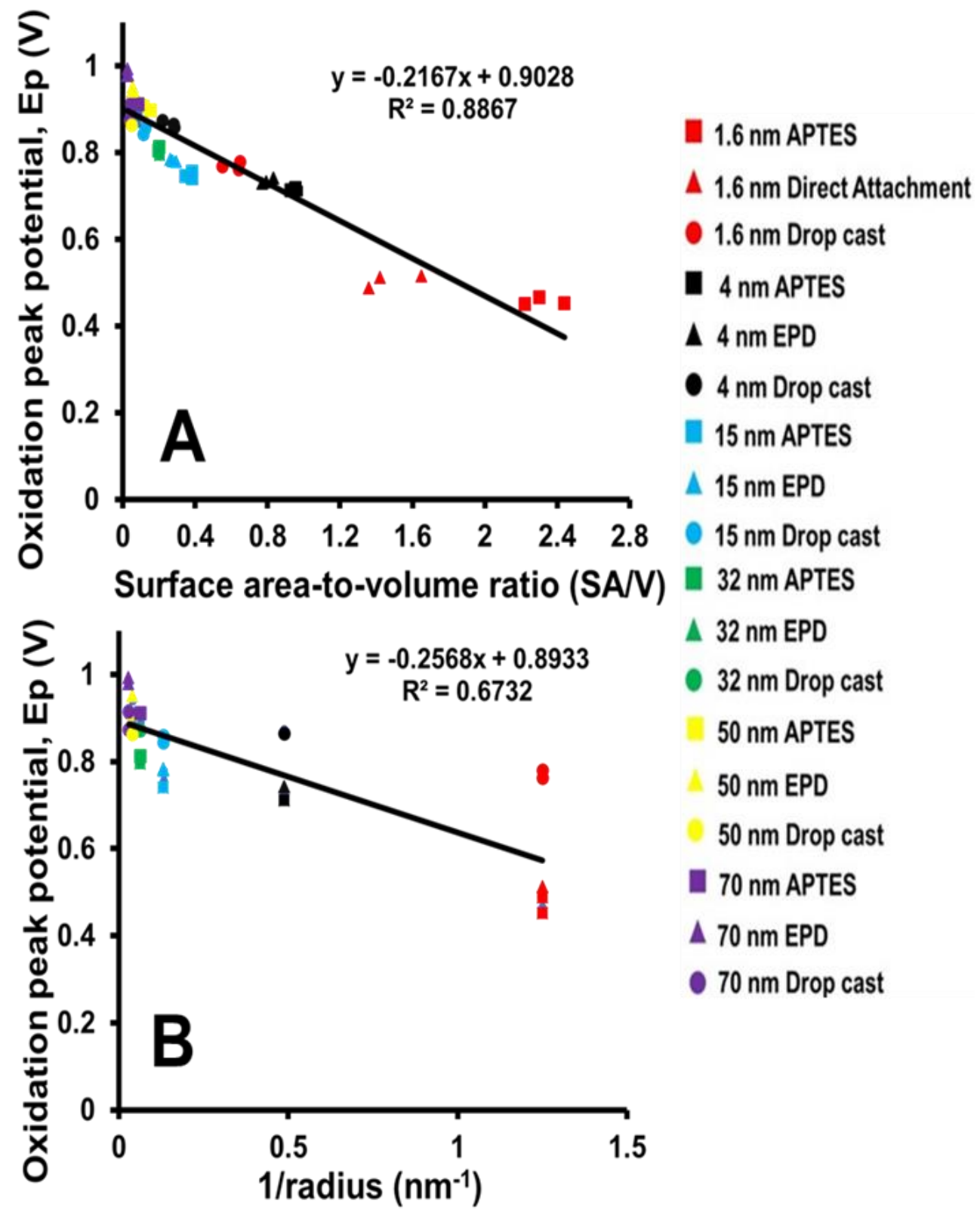


Figure 5. 7. Plots of $(A) E_{p, o x}$ vs. $S A / V$ and $(B) E_{p, o x} v s .1 /$ radius for the different sizes of Au NSs and different assembly methods in this study. The correlation is much better with electrochemically-measured SA/V.

The plot shows that $E_{p, o x}$ decreases linearly as $S A / V$ increases, regardless of the NS size and assembly method. The slope was found to be $-0.217 \mathrm{~V}-\mathrm{nm}$ and the $y$-intercept was $0.903 \mathrm{~V}$, which is close to the $E_{b, o x}$ of $A u$. The $R^{2}$ value of 0.89 indicated a reasonably good linear correlation. For these points we did not use samples that had the same overall Au coverage. Since Au coverage can also effect the $E_{p, o x}$ value, this likely also affected the correlation somewhat. Regardless, the trend is clear that $E_{p, o x}$ is inversely related to $S A / V$ with a linear trend.

We also plotted the $E_{p, o x}$ values for the different samples versus $1 /$ radius as determined by TEM and SEM. This should be provide a good linear inverse relation, as predicted by the Plieth equation (equation 3 below), relating the oxidation potential of metal NSs $\left(E_{p, o x}\right)$ to the radius $(r)$ of the NSs and the bulk metal oxidation potential $\left(\mathrm{E}_{\mathrm{b}, \mathrm{ox}}\right) .{ }^{148}$

$$
E_{p, o x}=\left(-\frac{2 r V_{m}}{Z F}\right)\left(\frac{1}{r}\right)+E_{b, o x}
$$

Here, $y$ is the surface stress of $\mathrm{Au}\left(1880 \mathrm{erg} \mathrm{cm}^{-2}\right), V_{m}$ is the molar volume of $\mathrm{Au}$ $\left(10.21 \mathrm{~cm}^{3} \mathrm{~mol}^{-1}\right), \mathrm{Z}$ is the number of electrons during oxidation of the metal, and $F$ is Faraday's constant. The Plieth equation shows that $E_{p, o x}$ is directly related to $1 / \mathrm{r}$ with a negative slope, but it does not do so well in actuality, as indicated by the worse $R^{2}$ value of 0.67 . If full access to the SA of the Au NS was allowed, then we expect much better correlation would occur with $1 /$ radius. Since that is not the 
case the plot fits better with the electrochemically-measured SA/V, which depends on the assembly method.

There are different explanations for the measured SA/V values for the NSs assembled by different methods as illustrated in Scheme I. In the case of APTESattached Au NSs,

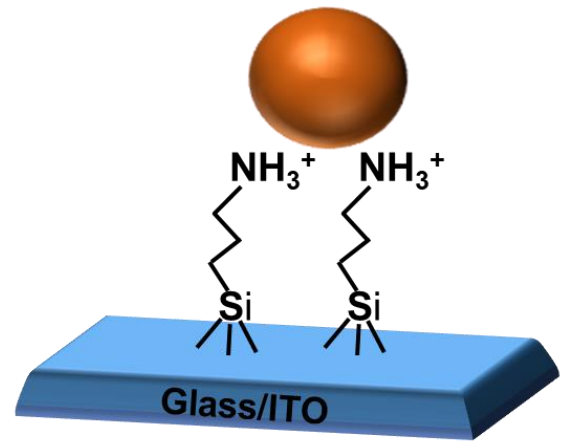

(A) NPs assembled by APTES linker

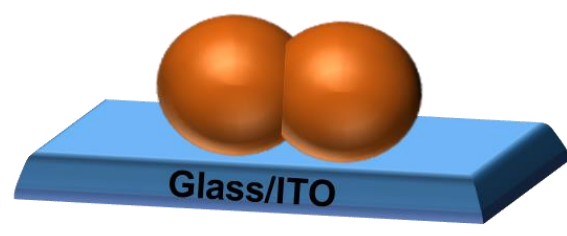

(C) NPs assembled by drop cast deposition

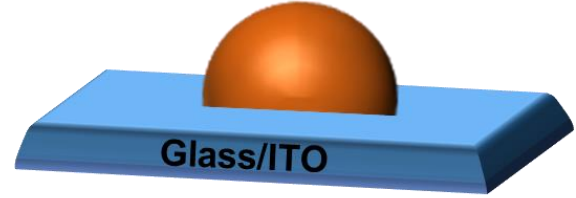

(B) NPs assembled by EPD

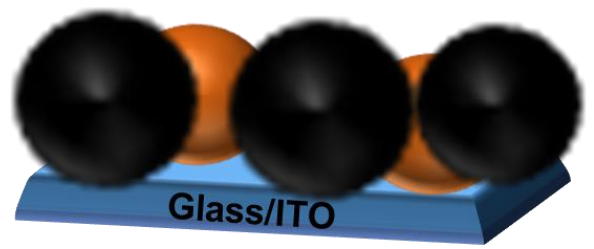

(D) NPs assembled by drop cast deposition after mixing with carbon black

Figure 5. 4. Schematic illustration of explanation for differences in SA/V of Au NSs for different assembly methods.

we believe the NSs are somewhat separated from the electrode by the linker and their full SA is accessible to the electrochemical environment. This leads to the highest SA values and highest SA/V (Figure 5.8, Case A). At the same time, they 
are also fully electronically connected because the linker is very short and not resisting charge transfer from ITO to the Au NS significantly. In contrast, for the EPD attached Au NSs, closer proximity of the NSs to the electrode surface decreases their SA possibly due to an increase in the surface contact between the NSs and the ITO electrode surface, which hinders access to some of the SA of the NS. ${ }^{195}$ The metal NS may even alter the shape slightly to become flattened or slightly hemispherical to accommodate the direct attachment to ITO (Figure 5.8, Case B). Not much is known about the metal NS/ITO contact interface, but it is clear that these NSs exhibit statistically significant lower SA/V and higher $E_{p, o x}$ values, especially for larger NSs. The SA/V also decreased for Au NSs assembled by drop-cast deposition with or without CB. This is due to strong interactions with other NSs and/or the CB, which again leads to a large decrease in accessible SA, therefore increasing $E_{p, o x}$ (Figure 5.8, Cases $C$ and $D$ ). 
As the SA/V of the Au NSs was found to be affected by their assembly methods, we also compared the effect of assembly method on the electrocatalytic activity of the Au NSs towards the oxygen reduction reaction (ORR). Theoretical and experimental studies have been performed on the ORR with small-sized Au NSs and the reaction mechanism is well known. ${ }^{196}$ In our work, we attached $4 \mathrm{~nm} \mathrm{Au}$ NSs on glass/ITO by APTES-linkage, EPD deposition and drop-cast deposition with all having similar Au coverages. We found that despite having similar coverages, as determined by integrating the stripping peak in ASV, the APTESassembled Au NSs showed higher ORR current than that of the EPD assembled and drop-cast deposited ones, clearly indicating the effect of the assembly method of NSs on their electrocatalytic performance (Figure 5.9). The different activities are due to the different available SA in each case. 

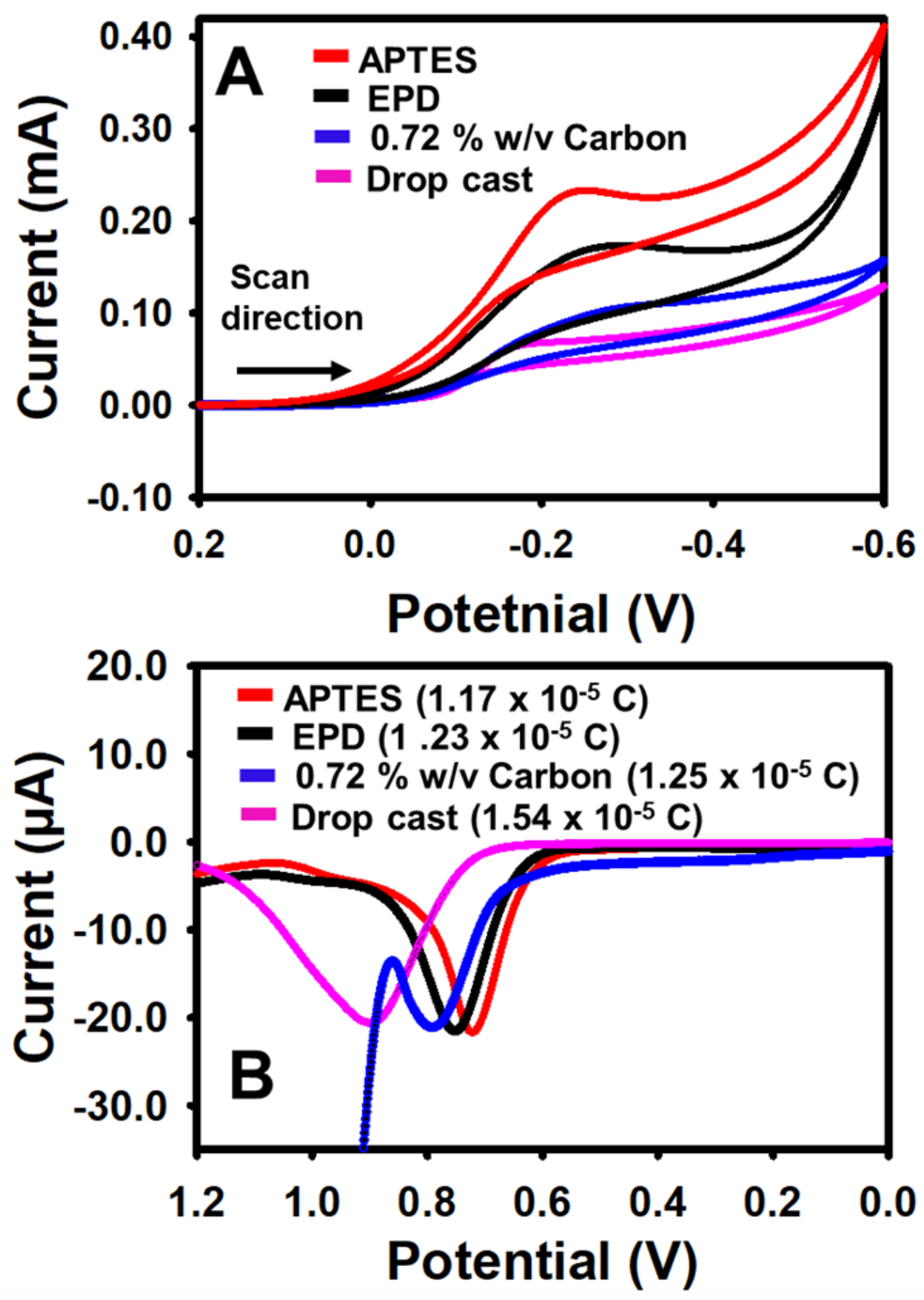

Figure 5. 9. (A) ORR for $4 \mathrm{~nm}$ Au NSs assembled by APTES linker, EPD and drop cast deposition with and without carbon black. (B) ASVs of the different Au NS in (A), showing similar Au coverages for all the assembly methods. 


\subsection{Conclusions}

In conclusion, this work provides important information about the effect of different assembly methods on the electrochemical SA/V of Au NSs in the 1.6 to $70 \mathrm{~nm}$ diameter size range. The electrochemical SA/V was largest for NSs assembled via APTES linker, followed by those assembled by EPD and then dropcast deposition with and without CB. The decrease in SA/V between the different assembly methods was more dramatic for $15 \mathrm{~nm}$ Au NSs than other sizes. The SA/V was significantly decreased for the drop-cast NSs due to aggregation as observed by SEM imaging. The SA/V for Au NSs with different CB concentrations were also found to be decreased significantly. The SA decrease is likely due to physical blockage of the surface $A u$ atoms by $C B$. We found that $E_{p, o x}$ shifts to higher values for NSs as the SA/V decreases. A strong linear inverse relation between $E_{p, o x}$ and $S A / V$ exists with much better correlation than with the measured NS radius, since the assembly method can alter the exposed SA for the same size NSs. The trend in exposed SA for the different assembly methods caused increased ORR activity for APTES-assembled $4 \mathrm{~nm}$ diameter Au NSs as compared to those assembled by EPD or drop-cast deposition with CB. The trend observed for the reaction was APTES assembled > EPD assembled > drop-cast with $0.036 \%$ $\mathrm{w} / \mathrm{v}$ of $\mathrm{CB}>$ drop-cast with $0.072 \% \mathrm{w} / \mathrm{v}$ of $\mathrm{CB}>$ drop-cast with $0.28 \% \mathrm{w} / \mathrm{v}$ of $\mathrm{CB}$ $>$ direct drop-cast without $\mathrm{CB}$. Due to the inverse relationship between $\mathrm{E}_{\mathrm{p}, \mathrm{ox}}$ and $\mathrm{SA} / \mathrm{V}$, as the activity of the NS increases (higher SA/V), the stability against oxidation decreases (decreasing $E_{p, o x}$ ). The information and techniques described here can be helpful in optimizing the assembly method for maximum activity and 
stability. It also provides new insight into the effect of curvature on metal oxidation potentials since these NSs do not have altered curvature, yet there oxidation potential varies significantly due to differences in SA/V. 


\section{CHAPTER VI}

\section{THE SURFACE AREA-TO-VOLUME RATIO AND OXIDATION POTENTIAL OF}

AUAG NANOSPHERES AFTER AG DEALLOYING

\subsection{Introduction}

Metal NPs (NPs) have recently gained tremendous attention due to their widespread applications in catalysis, ${ }^{197}$ sensing, ${ }^{198}$ and therapeutics. ${ }^{199}$ Usually smaller sized NPs show superior performance for applications in catalysis and sensing due to their high SA-to-volume ratio (SA/V). A decrease in the size of NPs produces an increasing number of potentially active high energy defect sites where some reactions are favored. ${ }^{200}$ Recently, porous nanostructures haven been explored as another type of nanomaterial potentially useful for highly efficient catalytic, ${ }^{201-202}$ sensing ${ }^{203}$ and energy storage applications. ${ }^{204}$ Similar to smaller sized NPs, these nanostructures possess high SA/V and are potentially better alternative to smaller-sized NPs for certain applications. There are different approaches available to form porous structures for different applications. With regard to nanoporous $\mathrm{Au}$, common fabrication methods include etching of $\mathrm{Au}$ electrodes, ${ }^{205}$ electrodeposition ${ }^{101}$ and selective dealloying of the less noble metal from alloys of Au. ${ }^{104,106}$ While the synthesis of high SA nanoporous Au involve approaches such as oxidative etching ${ }^{206-207}$ From amongst different approaches, selective dealloying has recently gained significant attention due to its specific benefits, such as the ability to control the pore size and morphology of the porous metal structures. 
Selective dealloying has been successfully used by researchers to synthesize porous Au nanostructures with useful properties and applications. Kim and coworkers ${ }^{106}$ synthesized highly porous Au core/porous shell NPs (CPS NPs) by selectively dissolving $\mathrm{Ag}$ atoms from $\mathrm{Au} / \mathrm{Au}-\mathrm{Ag}$ core/alloy shell NPs. The CPS NPs showed very high turnover frequency for 4-nitrophenol reduction due to their catalytically active ultra-porous shells, which contained many surface defects. Chen and coworkers ${ }^{201}$ selectively leached $\mathrm{Ag}$ from $\mathrm{AuAg}$ foils to form nanoporous $\mathrm{Au}$ (AuNPore). This nanoporous Au was found to be an efficient catalyst for selective diboration of methylenecyclopropanes due to a stable trimethylenemethane intermediate on the surface of nanoporus Au. Snyder and coworkers ${ }^{104}$ de-alloyed AgAu alloys in neutral $\mathrm{pH} \mathrm{AgNO}_{3}$ and in $\mathrm{HNO}_{3}$, both having high $\mathrm{SA}$ with great potential for catalysis and sensing applications. Zhang and coworkers ${ }^{105}$ prepared Au-based nanoboxes and nanocages having controlled porosity by dissolving $\mathrm{Ag}$ from Au-Ag alloy nanoboxes using $\mathrm{H}_{2} \mathrm{O}_{2}$. These dealloyed nanoboxes and nanocages were useful sensors to detect $\mathrm{H}_{2} \mathrm{O}_{2}$. Qi and coworkers ${ }^{208}$ dealloyed $\mathrm{Ag}$ from AuAg alloys and observed a porous nested network microstructure after the second dealloying step. They mentioned that these microstructures possessed a faster charge transport rate and could be useful in applications where the electrochemical properties need to be tuned. Chao and coworkers ${ }^{209}$ dealloyed Au-based metallic glass ribbon using iron chloride solution. The dealloying created fine nanoporous $\mathrm{Au}$ on the surface. These nanoporous structures contained surface-enhanced Raman hot spots within the fine interstices alongside the large SA regions, leading to multiple reflections of incident laser 
light. This resulted in low detection limits of $10^{-8} \mathrm{M}$ for $p$-ATP. Zhong and coworkers ${ }^{210}$ developed a sensitive non-enzymatic glucose sensor from porous $\mathrm{Au}$ networks (HPANs), which acted as an electrocatalyst for glucose oxidization. Xia and coworkers used selective dealloying of Ag from AuAg alloy nanoboxes using aqueous $\mathrm{Fe}\left(\mathrm{NO}_{3}\right)_{3}$ or $\mathrm{NH}_{4} \mathrm{OH}$ as etchant to form porous nanocages and nanoboxes with tunable surface plasmon resonance properties suitable for different applications.

Considering the usefulness of these porous Au nanostructures, it is critical to accurately measure their SA in order to correlate it with their catalytic and sensing efficiencies. For example, Vega and coworkers ${ }^{211}$ determined the SA of nanoporous metals formed by electrochemical dealloying of Ag-Au-Pt by using the Brunauer-Emmett-Teller (BET) method. Rouya and coworkers ${ }^{212}$ measured the SA of nanoporous gold films having thicknesses up to $930 \mathrm{~nm}$ and pores on the order of tens of nanometers in size using different electrochemical methods, such as Cu underpotential deposition (UPD), surface Au oxidation/reduction reaction, and electrochemical impedance spectroscopy (EIS). The SA increased linearly with increasing thickness. The SA measurement for porous Au nanostructures is not sufficient information to fully assess the usefulness and properties in terms of activity and stability. One needs to know the volume (V) in order to determine the overall amount of material being used as this determines the cost. SA/V is also a critical parameter that determines the stability of the metal against oxidation and ripening processes, which can lead to structural and size changes. 
We recently developed a simple electrochemical method to measure the SA/V of Au nanostructures, using Au NSs as a model metal to test the method. We used this ratio to successfully analyze the size $e^{88,213}$ and aggregation ${ }^{156,181}$ of Au NSs and also to determine the effect of different electrode assembly methods on the SA/V and stability ${ }^{178}$ of these Au nanostructures. In this work, we aim to use our electrochemical method to measure the SA/V of porous Au nanostructures formed by the selective dealloying of Ag from electrode-assembled AuAg alloy NPs and correlate the measured SA/V to the initial alloy composition and their eventual stability against oxidation. We believe that the electrochemical SA/V measurements of these porous Au NSs directly on the electrode support will be highly beneficial over other methods due to the simplicity, speed, and ability to perform the measurement right on the electrode. The information will be highly useful for quickly assessing synthetic methods for preparing porous structures and correlating the SA/V to their function and stability.

\subsection{Experimental Section}

The chemicals, alloy synthesis and dealloying procedures, electrode assembly method, ozone cleaning procedure, optical characterization, microscopic characterization, electrochemical SA/V assessment, and oxygen reduction reaction monitoring are described in the experimental procedures in Chapter II.

\subsection{Results and Discussion}


We synthesized mixed AuAg alloy NSs having different contents of Ag $(50 \%, 75 \%$ and $90 \%)$ using the procedures reported by El-Sayed and coworkers. ${ }^{214}$ We made slight modifications in the synthesis procedure where we used a lower concentration of trisodium citrate as that reported in their work. This led to bigger AuAg alloy NPs than their work for different percentages of Au and $\mathrm{Ag}$ in the alloys, which is similar to the trend observed in the case of Au NSs. ${ }^{215}$ We also synthesized intact Au NSs using a similar procedure, excluding $\mathrm{Ag}^{+}$from the reaction. The UV vis spectra matched well with the alloy compositions and blue shifted with increasing percentages of Ag as observed in Figure 6.1 (red, blue and green spectra) compared to the pure Au NSs synthesized by the same method (black spectrum). The UV-vis confirmed the successful synthesis 

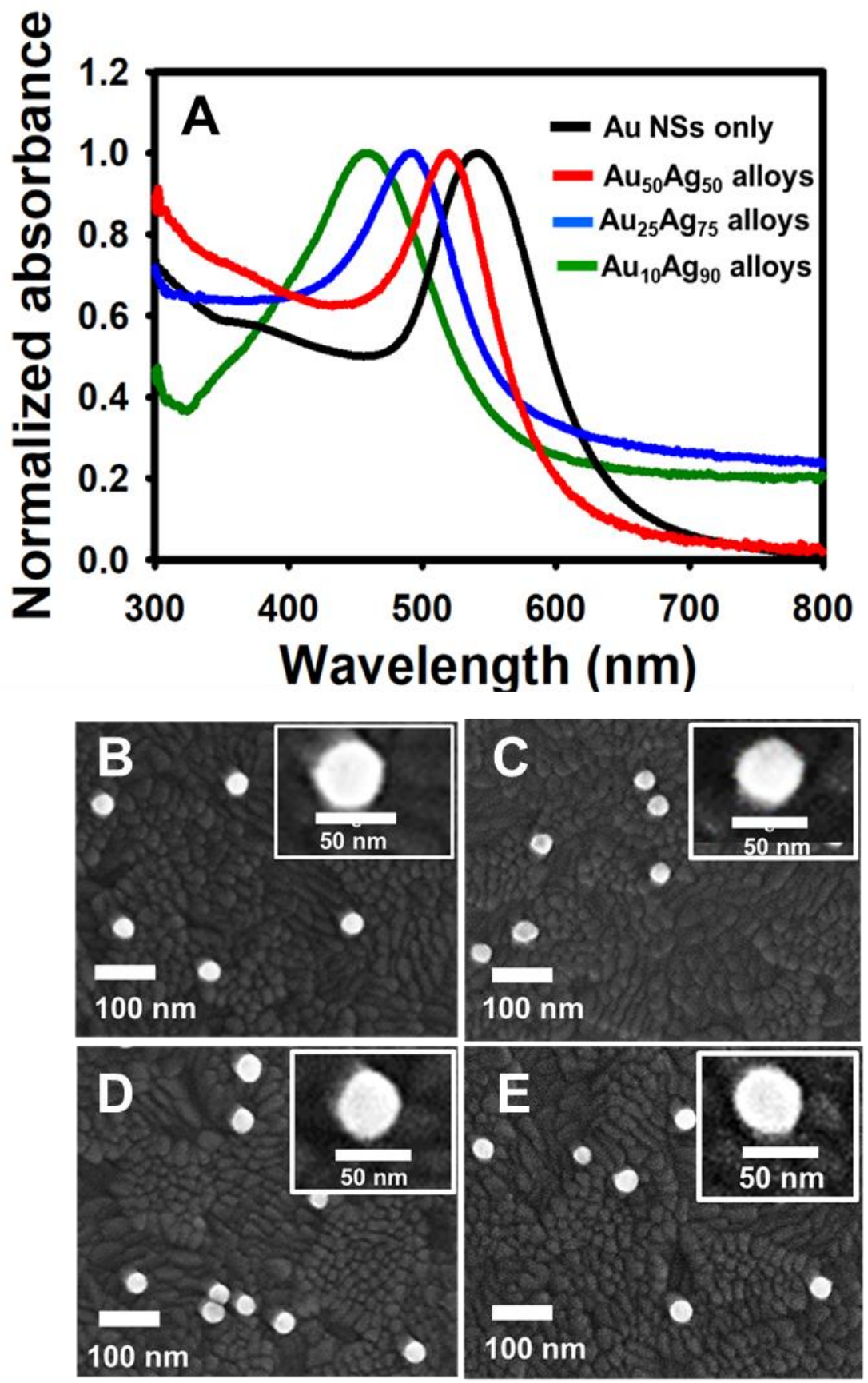

Figure 6. 1. UV vis spectra (A) and SEM images of Au NSs (B) and Au50Ag50, $\mathrm{Au}_{25} \mathrm{Ag}_{75}$ and $\mathrm{Au}_{10} \mathrm{Agg}_{90}(\mathrm{C}$ to $\mathrm{E}$ ) alloys by modification in the method by El Sayed. 
The as-synthesized AuAg alloys were then subjected to selective dealloying of Ag. Figure 6.2 shows the steps involved for the dealloying procedure. First, AuAg alloys having different $\mathrm{Ag}$ content were synthesized (step 1). Following this, the AuAg alloys were electrostatically attached to glass/ITO/APTES electrodes by simple soaking (step 2). Next, the samples were dealloyed by soaking the samples in $5 \mathrm{~mL}$ of $200 \mathrm{mM} \mathrm{Fe}\left(\mathrm{NO}_{3}\right)_{3} \cdot 9 \mathrm{H}_{2} \mathrm{O}$ for 3 hours (step 3). Following this, an ASV scan was performed from 0.0 to $0.5 \mathrm{~V}$ to check if any $\mathrm{Ag}$ was left. We also

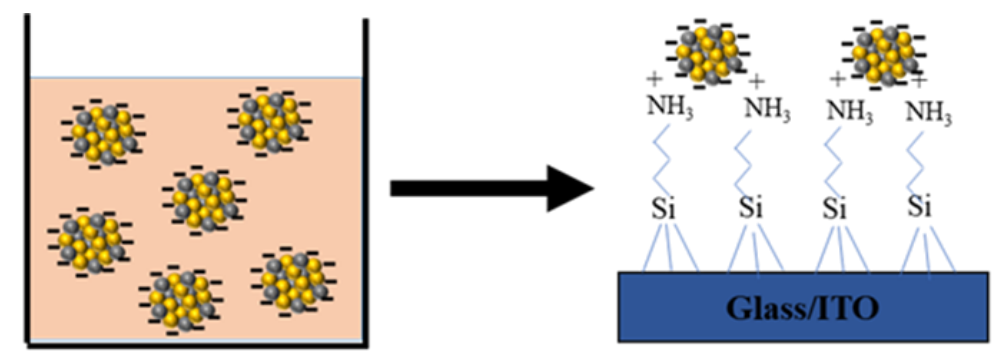

Step 1: Synthesis of AuAg alloys

Step 2: Electrostatic attachment of AuAg alloys to glass/lTO
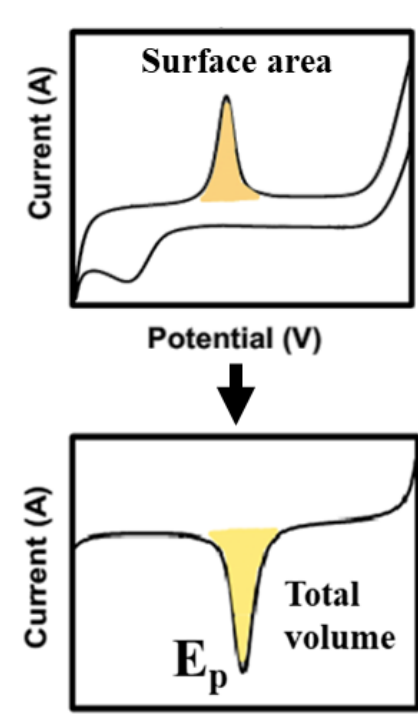

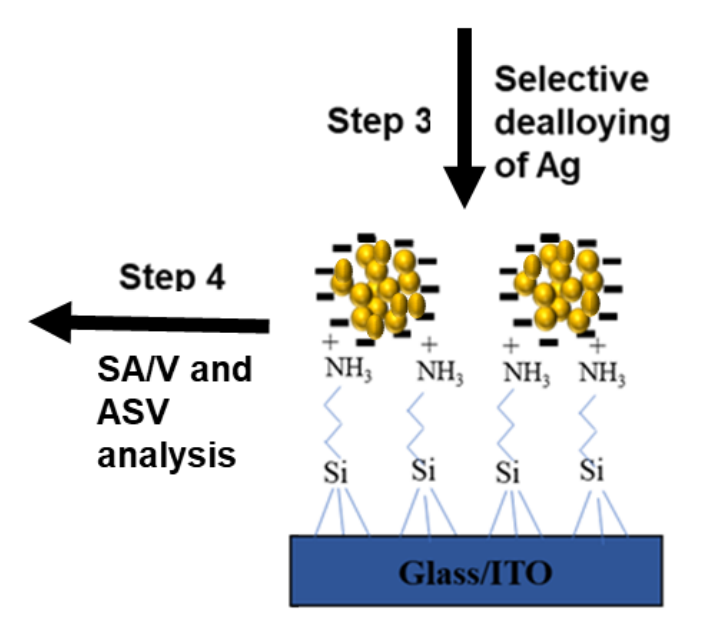

Figure 6. 2. Scheme for general experimental work involved in this work. 
confirmed complete oxidative dissolution of $\mathrm{Ag}$ by $\mathrm{Fe}\left(\mathrm{NO}_{3}\right)_{3} \cdot 9 \mathrm{H}_{2} \mathrm{O}$ by a control experiment wherein we soaked $45 \mathrm{~nm}$ diameter Ag NPs in $200 \mathrm{mM} \mathrm{Fe}\left(\mathrm{NO}_{3}\right)_{3} \cdot 9 \mathrm{H}_{2} \mathrm{O}$ for 3 hours and observed that under this dealloying condition all the Ag NPs completely dissolved (Figure 6.3, red and blue plots). After dealloying Ag from the respective AuAg alloys, the remaining Au NSs on glass/ITO samples were treated with ozone for 16 minutes to clean the sample off any impurities. We also treated the intact Au NSs by ozone. The samples were then subjected to cyclic voltammetry $(\mathrm{CV})$ in $0.1 \mathrm{M} \mathrm{HClO}_{4}$ to measure the $\mathrm{SA}$ and anodic stripping voltammetry (ASV) in $0.01 \mathrm{M} \mathrm{KBr}$ in $0.1 \mathrm{M} \mathrm{KClO}_{4}$ to measure the $\mathrm{E}_{\mathrm{p}}$ and $\mathrm{V}$ of the Au nanostructures as described previously. ${ }^{88}$ 


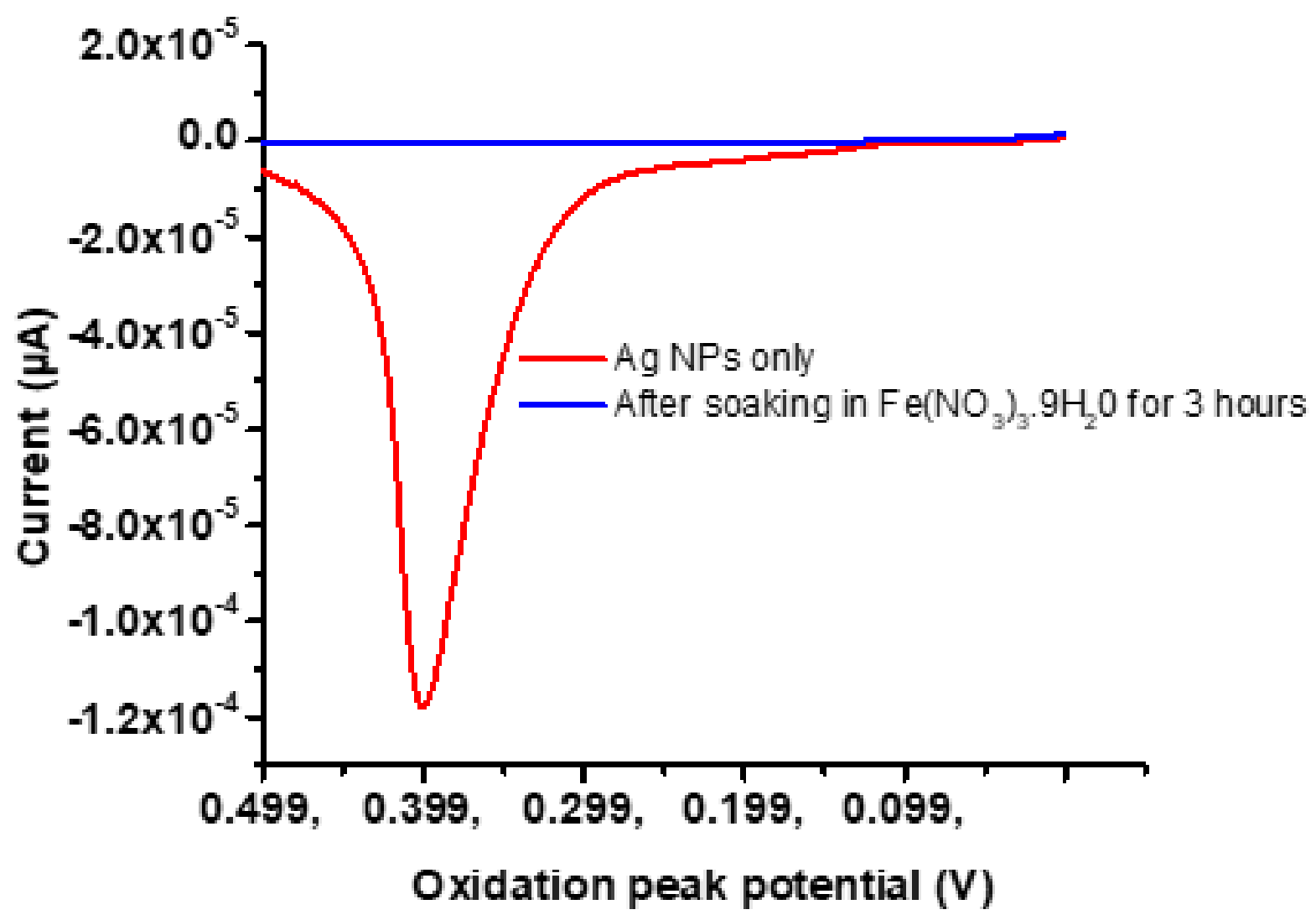

Figure 6. 3. ASVs of $50 \mathrm{~nm} \mathrm{Ag} \mathrm{NPs} \mathrm{attached} \mathrm{to} \mathrm{glass/ITO/APTES} \mathrm{without} \mathrm{further}$ treatment (red plot) and after soaking in $200 \mathrm{mM} \mathrm{Fe}\left(\mathrm{NO}_{3}\right)_{3} \cdot 9 \mathrm{H}_{2} \mathrm{O}$ for 3 hours (blue plot). 
Figure 6.4 shows CVs (top) and ASVs (bottom) of pure Au NSs (blue plots) and nanoporous $\mathrm{Au}$ NPs obtained by dealloying $\mathrm{Ag}$ from $\mathrm{Au}_{50} \mathrm{Ag}_{50}, \mathrm{Au}_{25} \mathrm{Ag}_{75}$, and $\mathrm{Au}_{10} \mathrm{Ag}_{90}$ alloy NPs. All of the NPs were attached to glass/ITO/APTES electrodes and had outer diameters of $\sim 50 \mathrm{~nm}$. These Au NSs have similar ASV volumes based on the $\sim 2.8 \times 10^{-4} \mathrm{C}$ of charge obtained from integrating the oxidation peak in the ASVs (bottom plots). It is clear from the CVs then that the SA/V increased as the content of $\mathrm{Ag}$ in the NPs increased before dealloying. Pure Au NSs had a SA of $2.65 \times 10^{-6} \mathrm{C}$ (blue graph) while the SAs of the nanoporous NPs formed by dealloying $\mathrm{Au}_{50} \mathrm{Ag}_{50}, \mathrm{Au}_{25} \mathrm{Agg}_{75}$, and $\mathrm{Au}_{10} \mathrm{Ag} 90$ were $5.30 \times 10^{-6} \mathrm{C}$ (green graph), $8.85 \times 10^{-6} \mathrm{C}$ (red graph), and $13.4 \times 10^{-6} \mathrm{C}$ (black graph), respectively. With similar volumes, the SA/V followed the order of $\mathrm{Au}_{10} \mathrm{Agg}_{90}>\mathrm{Au}_{25} \mathrm{Ag}_{75}>\mathrm{Au}_{10} \mathrm{Ag}_{90}>\mathrm{Au}$. Also, the nanoporous Au obtained by dealloying showed a negative shift in $E_{p}$ compared to the intact Au NSs. This negative shift increased with increasing $\mathrm{Ag}$ content before dealloying. As observed in Figure 6.3B, the $E_{p}$ was $0.92 \mathrm{~V}$ for 50 nm pure Au NSs (black graph), $0.85 \mathrm{~V}$ for dealloyed Au50Ag50 NPs (green graph), $0.80 \mathrm{~V}$ for dealloyed $\mathrm{Au}_{25} \mathrm{Ag}_{75} \mathrm{NPs}$ (red graph) and $0.76 \mathrm{~V}$ for dealloyed $\mathrm{Au}_{10} \mathrm{Agg}_{90}$ NPs (black graph). As observed with different sized Au NSs in Chapter III and Au NSs assembled in different ways in Chapter V, the oxidation properties $\left(E_{p}\right)$ of $A u$ nanostructures is determined by the SA/V. 

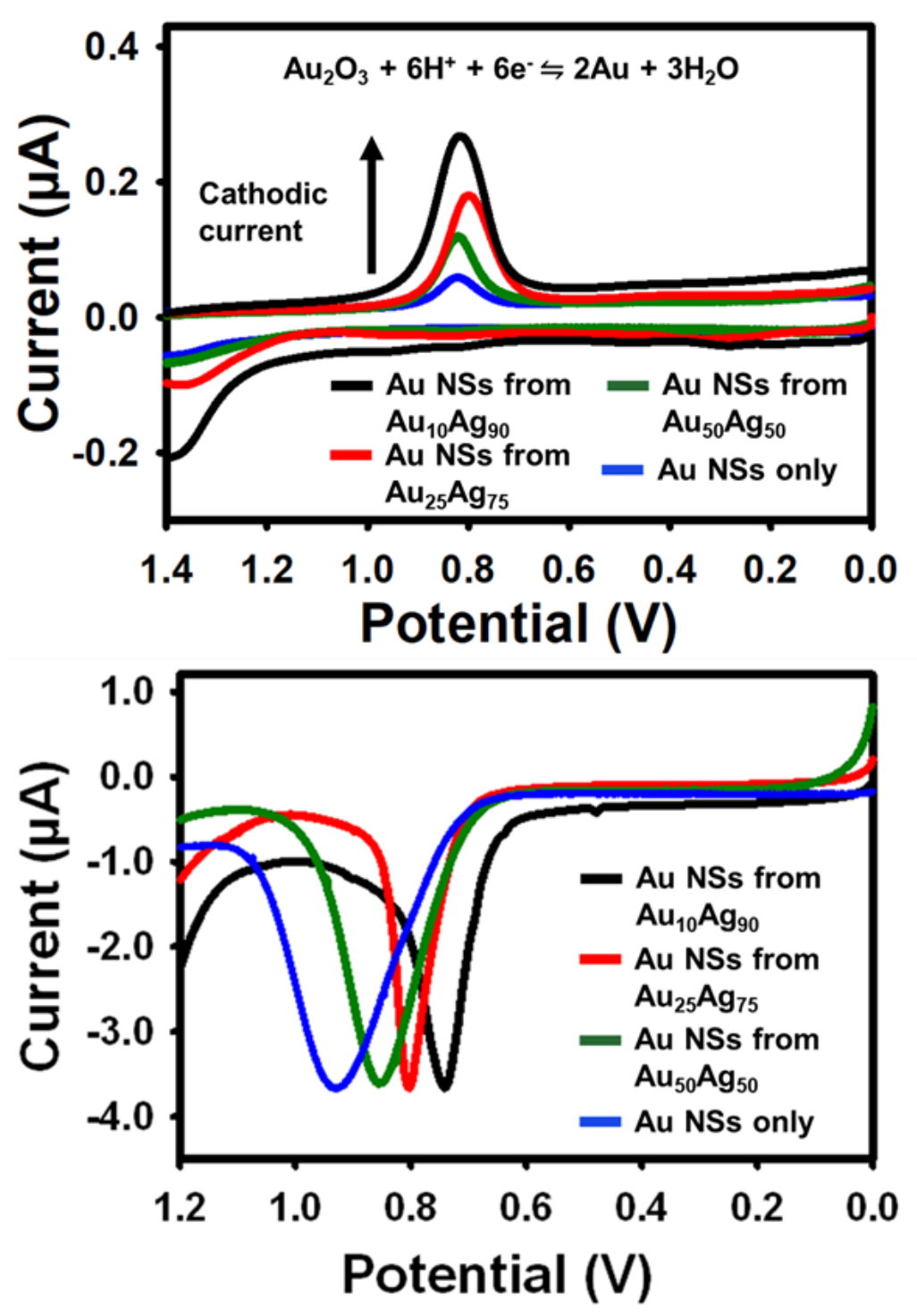

Figure 6. 4. CVs (top) and ASVs (bottom) of $\sim 50 \mathrm{~nm}$ diameter pure Au NSs (blue plots) and nanoporous $\mathrm{Au}$ NSs prepared by dealloying the $\mathrm{Ag}$ from $\mathrm{Au}_{50} \mathrm{Ag}_{50}$ (green plots), $\mathrm{Au}_{25} \mathrm{Ag}_{75}$ (red plots), and $\mathrm{Au}_{10} \mathrm{Agg}_{90}$ (black plots). 
In order to better understand the change in SA and SA/V for the Au NSs obtained by dealloying Ag from the AuAg alloy NSs, we obtained SEM images of all samples before and after dealloying. From the SEM imaging and size analysis based on it, we observed two important things about the dealloyed nanoporous Au NSs. First, the outer diameter of the nanoporous Au NSs were within the standard deviation of the diameter of the AuAg alloy NSs before dealloying and the intact Au NSs. Second, the nanoporous samples of dealloyed Au NSs contained distorted, flattened and porous Au NSs as seen in Figure 6.5. These distorted, flattened, and
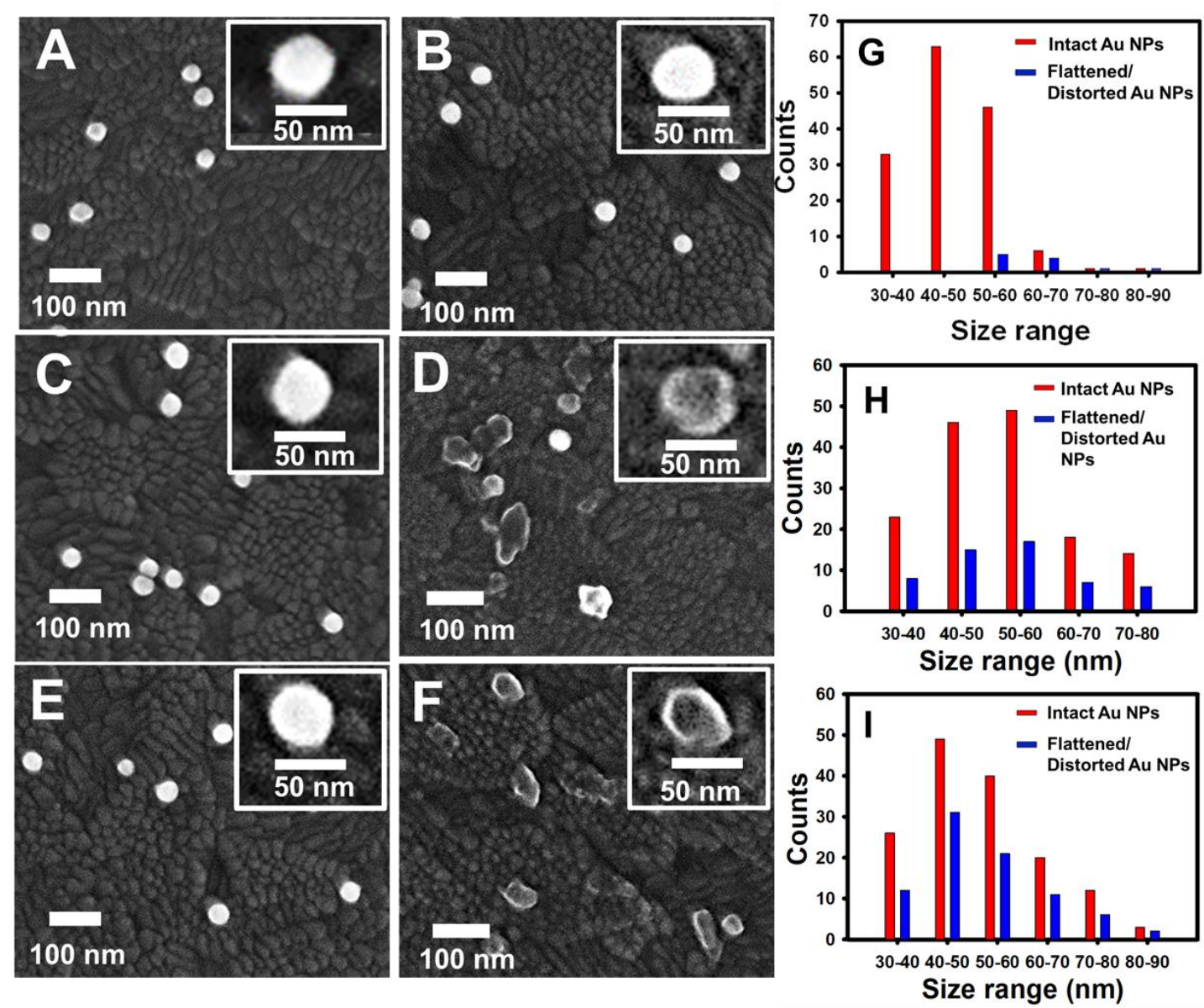

Figure 6. 5. SEM images of $A_{50} A_{50}$ alloy NPs $(A, B), A_{75} A_{25}(C, D)$, and $\mathrm{Au}_{10} \mathrm{Agg}_{90}(\mathrm{E}, \mathrm{F})$ before $(\mathrm{A}, \mathrm{C}, \mathrm{E})$ and after $(\mathrm{B}, \mathrm{D}, \mathrm{F})$ dealloying and the corresponding 
histograms $(G, H, I)$. The histograms show the number of intact Au NPs and number of porous/flattened/distorted NPs for each size range based on the SEM images.

porous Au NSs constitute $35 \%$ and $55 \%$ of the total population of Au NSs for $\mathrm{Au}_{25} \mathrm{Ag}_{75}$ and $\mathrm{Au}_{10} \mathrm{Ag}_{90}$ alloys, respectively (Figure 6.5, $\mathrm{H}$ and I). Figure 6.6 shows expanded images of some representative individual $\mathrm{Au}_{25} \mathrm{Ag}_{75}$ alloy NSs before (Figure 6.6, $A$ and $B$ ) and after (Figure 6.6,C -H) dealloying.
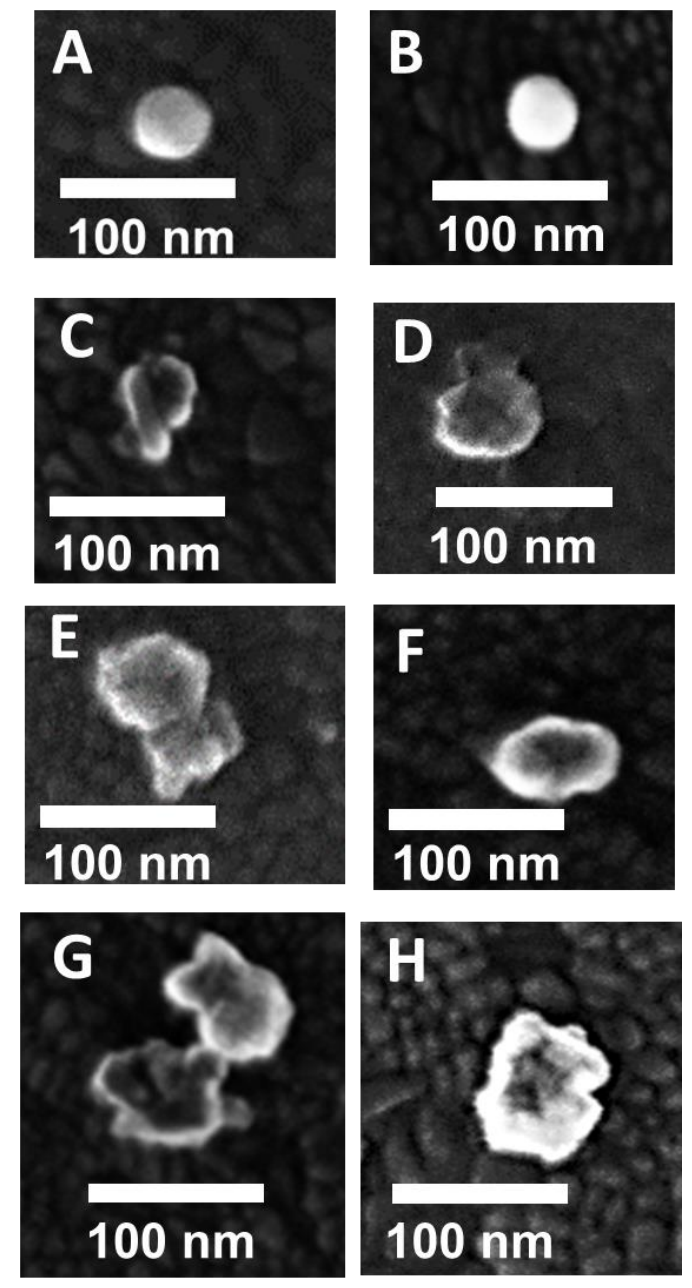
Figure 6. 6. SEM images of individual $A u_{25} A_{75}$ alloy NSs ( $A$ and $\left.B\right)$ and $A u$ NSs obtained after dealloying ( $\mathrm{C}$ to $\mathrm{H})$

The zoom in view of the dealloyed NSs clearly show the porous, flattened and distorted morphology after dealloying, which is responsible for the increased SA/V. The morphology is similar to that observed by Sieradzki and coworkers. ${ }^{32}$ We did not observe a significant number (only about $6 \%$ ) of such distorted and flattened Au NSs after dealloying of Au50Ag50 alloys. A reason for this is that $50 \% \mathrm{Ag}$ is below the 'parting limit' for Ag in AuAg alloys to form porous nanostructures. The minimum amount of $\mathrm{Ag}$ needed is $55 \%$ of the alloy composition to form porous nanostructures after dealloying. ${ }^{32,216}$ However the electrochemical SA/V was still found to be higher as compared to the intact ones, showing the high sensitivity of our electrochemical technique to detect this small increase in SA and negative shift in $E_{p}$ for the Au NSs obtained from these alloys. Table 6.1 provides all of the data for the relevant SEM measured sizes of the different Au and AuAg alloy NSs before and after dealloying along with the electrochemically-measured $S A / V$ and $E_{p}$ (ave. \pm std. dev.). 
Table 6. 1. SEM determined outer diameter (ave \pm std dev) of Au and AuAg alloy NSs before and after dealloying AuAg alloys, SA/V (ave \pm std dev) and $E_{p}$ (ave \pm std dev) of the pure Au NSs and AuAg alloy NSs after dealloying

\begin{tabular}{|c|c|c|c|c|}
\hline $\begin{array}{l}\text { Type of } \\
\text { NSs and } \\
\text { alloys }\end{array}$ & $\begin{array}{c}\text { SEM } \\
\text { determined } \\
\text { outer } \\
\text { diameter of } \\
\text { AuAg } \\
\text { alloys }(\mathrm{nm})\end{array}$ & $\begin{array}{l}\text { SEM determined } \\
\text { outer diameter } \\
\text { (ave } \pm \text { std dev) } \\
\text { of Au NSs } \\
\text { obtained by } \\
\text { dealloying AuAg } \\
\text { alloys (nm) }\end{array}$ & $\begin{array}{l}\text { SAV (ave } \pm \\
\text { std dev) of } \\
\text { the Au NSs } \\
\text { obtained by } \\
\text { dealloying } \\
(\mathrm{nm})\end{array}$ & $\begin{array}{l}E_{p} \text { (ave } \pm \text { std } \\
\text { dev) of the Au } \\
\text { NSs obtained } \\
\text { by dealloying } \\
\text { (nm) }\end{array}$ \\
\hline Au only & $50.73 \pm 7$ & - & $0.116 \pm 0.021$ & $0.924 \pm 0.032$ \\
\hline $\mathrm{Au}_{50} \mathrm{Ag}_{50}$ & $49.64 \pm 5.4$ & $53.29 \pm 7.30$ & $0.262 \pm 0.026$ & $0.854 \pm 0.016$ \\
\hline $\mathrm{Au}_{25} \mathrm{Ag}_{75}$ & $48.76 \pm 6$ & $53.54 \pm 10$ & $0.343 \pm 0.030$ & $0.805 \pm 0.023$ \\
\hline $\mathrm{Au}_{10} \mathrm{Ag}_{90}$ & $47.64 \pm 7$ & $51.96 \pm 11.84$ & $0.499 \pm 0.024$ & $0.752 \pm 0.013$ \\
\hline
\end{tabular}

Table 6.2. provides the individual CV determined SAs (in Coulombs), ASV measured volumes (in Coulombs), the SA-to-volume ratio (SA/V), average SA/V \pm std. dev., oxidation peak potentials $\left(E_{p}\right)$ and average $E_{p} \pm$ std. dev. As seen in the table, we obtained increasing SA/V and negative shift in $E_{p}$ for Au NSs obtained by dealloying AuAg alloys compared to similar-sized intact Au NSs. 
Table 6. 2. Au NSs type, CV measured SA, ASV measured volume, SA-to-volume ratio (SA/V), average $S A / V \pm$ std. dev., oxidation peak potential $\left(E_{p}\right)$ and average $E_{p} \pm$ std. dev.

\begin{tabular}{|c|c|c|c|c|c|c|}
\hline $\begin{array}{c}\text { Au NSs } \\
\text { type }\end{array}$ & $\begin{array}{c}\text { CV } \\
\text { Measured } \\
\text { SA } \\
\text { (Coulomb } \\
\text { s) }\end{array}$ & $\begin{array}{c}\text { ASV } \\
\text { Measured } \\
\text { Volume } \\
\text { (Coulomb } \\
\text { s) }\end{array}$ & $\begin{array}{c}\text { SA-to- } \\
\text { Volume } \\
\text { Ratio } \\
\text { (SA/V) }\end{array}$ & $\begin{array}{c}\text { Average } \\
\text { SA/V } \pm \\
\text { Std. } \\
\text { Dev. }\end{array}$ & $\begin{array}{l}\text { Oxidatio } \\
\text { n peak } \\
\text { potential } \\
\text { (Ep) }\end{array}$ & $\begin{array}{c}\text { Average } \\
\text { Ep } \pm \\
\text { Std. } \\
\text { Dev. }\end{array}$ \\
\hline \multirow{3}{*}{$\begin{array}{c}50 \mathrm{~nm} \\
\text { APTES } \\
\text { ozone }\end{array}$} & $\begin{array}{c}2.65 \times 10- \\
6\end{array}$ & $2.78 \times 10-5$ & 0.095 & \multirow[t]{3}{*}{$\begin{array}{c}0.116 \pm \\
0.021\end{array}$} & 0.931 & \multirow[t]{3}{*}{$\begin{array}{c}0.924 \pm \\
0.032\end{array}$} \\
\hline & $\begin{array}{c}7.52 \times 10- \\
6\end{array}$ & $5.44 \times 10-5$ & 0.138 & & 0.954 & \\
\hline & $\begin{array}{c}8.67 \times 10- \\
6\end{array}$ & $7.45 \times 10-5$ & 0.116 & & 0.889 & \\
\hline \multirow{3}{*}{$\begin{array}{c}\text { Porous } \\
\text { Au NSs } \\
\text { from } \\
\text { dealloyi } \\
\text { ng of } \\
\text { Au50Ag } \\
50\end{array}$} & $\begin{array}{c}5.83 \times 10- \\
6\end{array}$ & $2.62 \times 10-5$ & 0.222 & \multirow[t]{3}{*}{$\begin{array}{c}0.244 \pm \\
0.026\end{array}$} & 0.864 & \multirow[t]{3}{*}{$\begin{array}{c}0.854 \pm \\
0.016\end{array}$} \\
\hline & $\begin{array}{c}5.30 \times 10- \\
6\end{array}$ & $2.22 \times 10-5$ & 0.238 & & 0.832 & \\
\hline & $\begin{array}{c}1.66 \times 10- \\
5\end{array}$ & $6.05 \times 10-5$ & 0.274 & & 0.840 & \\
\hline \multirow{3}{*}{$\begin{array}{c}\text { Porous } \\
\text { Au NSs } \\
\text { from } \\
\text { dealloyi } \\
\text { ng of } \\
\text { Au25Ag } \\
75\end{array}$} & $\begin{array}{c}1.46 \times 10- \\
5\end{array}$ & $4.40 \times 10-5$ & 0.331 & \multirow[t]{3}{*}{$\begin{array}{c}0.343 \pm \\
0.030\end{array}$} & 0.821 & \multirow[t]{3}{*}{$\begin{array}{c}0.805 \pm \\
0.023\end{array}$} \\
\hline & $\begin{array}{c}8.85 \times 10- \\
6\end{array}$ & $2.71 \times 10-5$ & 0.324 & & 0.786 & \\
\hline & $1.54 \times 10^{-6}$ & $4.11 \times 10^{-5}$ & 0.374 & & 0.817 & \\
\hline \multirow{3}{*}{$\begin{array}{l}\text { Porous } \\
\text { Au NSs } \\
\text { from } \\
\text { dealloyi } \\
\text { ng of } \\
\text { Au10Ag } \\
90\end{array}$} & $\begin{array}{c}1.94 \times 10- \\
6\end{array}$ & $4.07 \times 10-6$ & 0.476 & \multirow[t]{3}{*}{$\begin{array}{c}0.499 \pm \\
0.024\end{array}$} & 0.749 & \multirow[t]{2}{*}{$\begin{array}{c}0.752 \pm \\
0.013\end{array}$} \\
\hline & $\begin{array}{c}3.67 \times 10- \\
6\end{array}$ & $1.42 \times 10-5$ & 0.533 & & 0.767 & \\
\hline & $\begin{array}{c}1.34 \times 10- \\
5\end{array}$ & $2.74 \times 10-5$ & 0.489 & & 0.741 & \\
\hline
\end{tabular}


Finally, the formation of distorted-flattened-porous Au NSs from AuAg alloys was also confirmed by red shift in UV vis spectra for these Au NSs as compared to their alloy counterpart. Figure 6.7. show the red shift in UV vis for Au NSs formed from Au25 $\mathrm{Ag} 75$ alloys on glass/lTO after dealloying with $\mathrm{Fe}\left(\mathrm{NO}_{3}\right)_{3} .9 \mathrm{H}_{2} \mathrm{O}$.

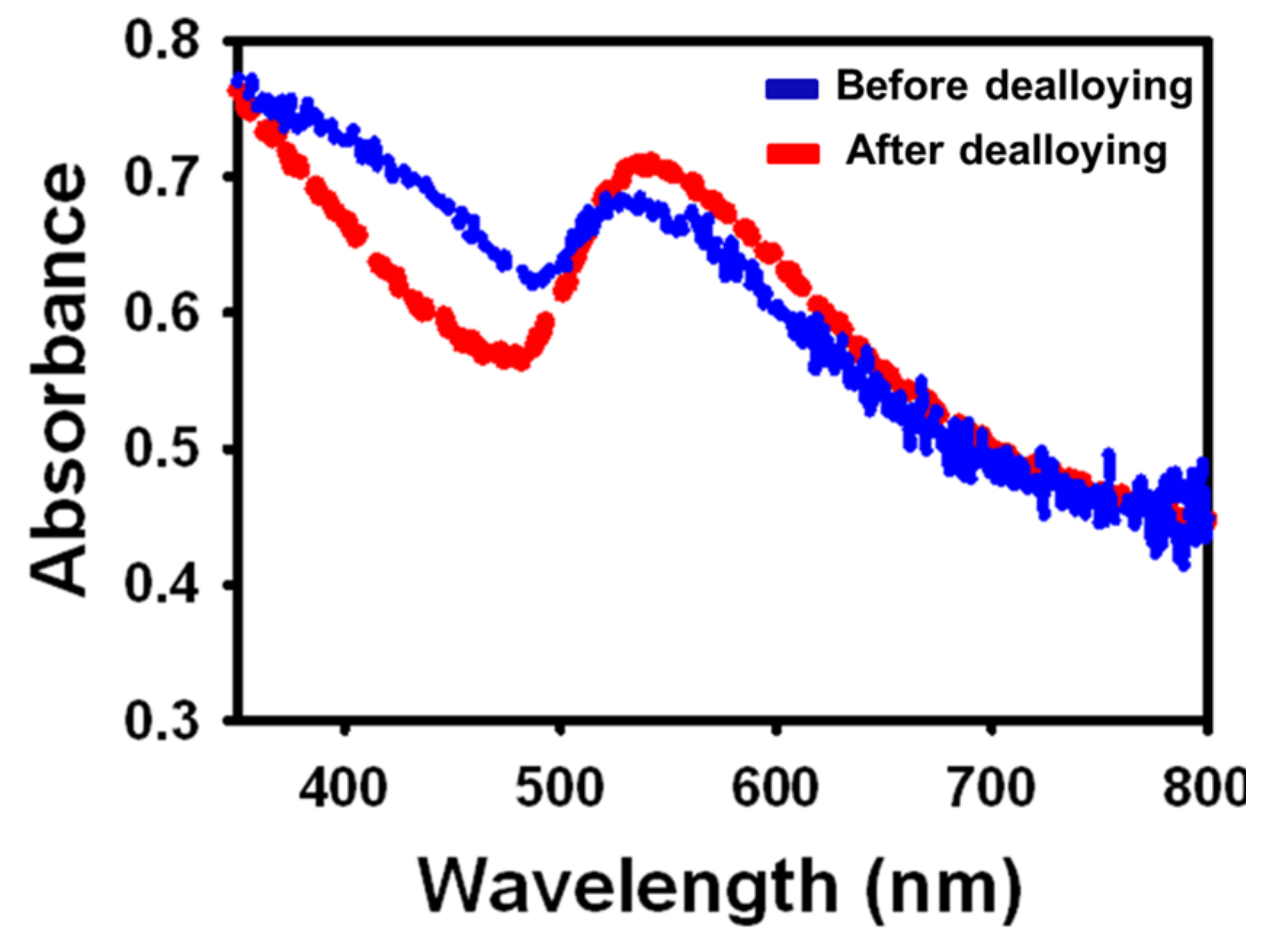

Figure 6. 7. UV vis spectra of Au25Ag75 alloys on glass/lTO before dealloying (blue spectra) and after dealloying (red spectra)

We also plotted the electrochemically-determined SA/V and $E_{p}$ of the Au NSs obtained by dealloying of AuAg alloys as function of \%Ag (Figure 6.8, A and B). The SA/V increased in the Au NSs obtained from AuAg alloys having increasing percentages of $\mathrm{Ag}$ in them (Figure 6.8A) as mentioned previously. Conversely, the 
$E_{p}$ decreased for $\mathrm{Au}$ NSs obtained from AuAg alloys having increasing percentages of $\mathrm{Ag}$ in them (Figure 6.8B).

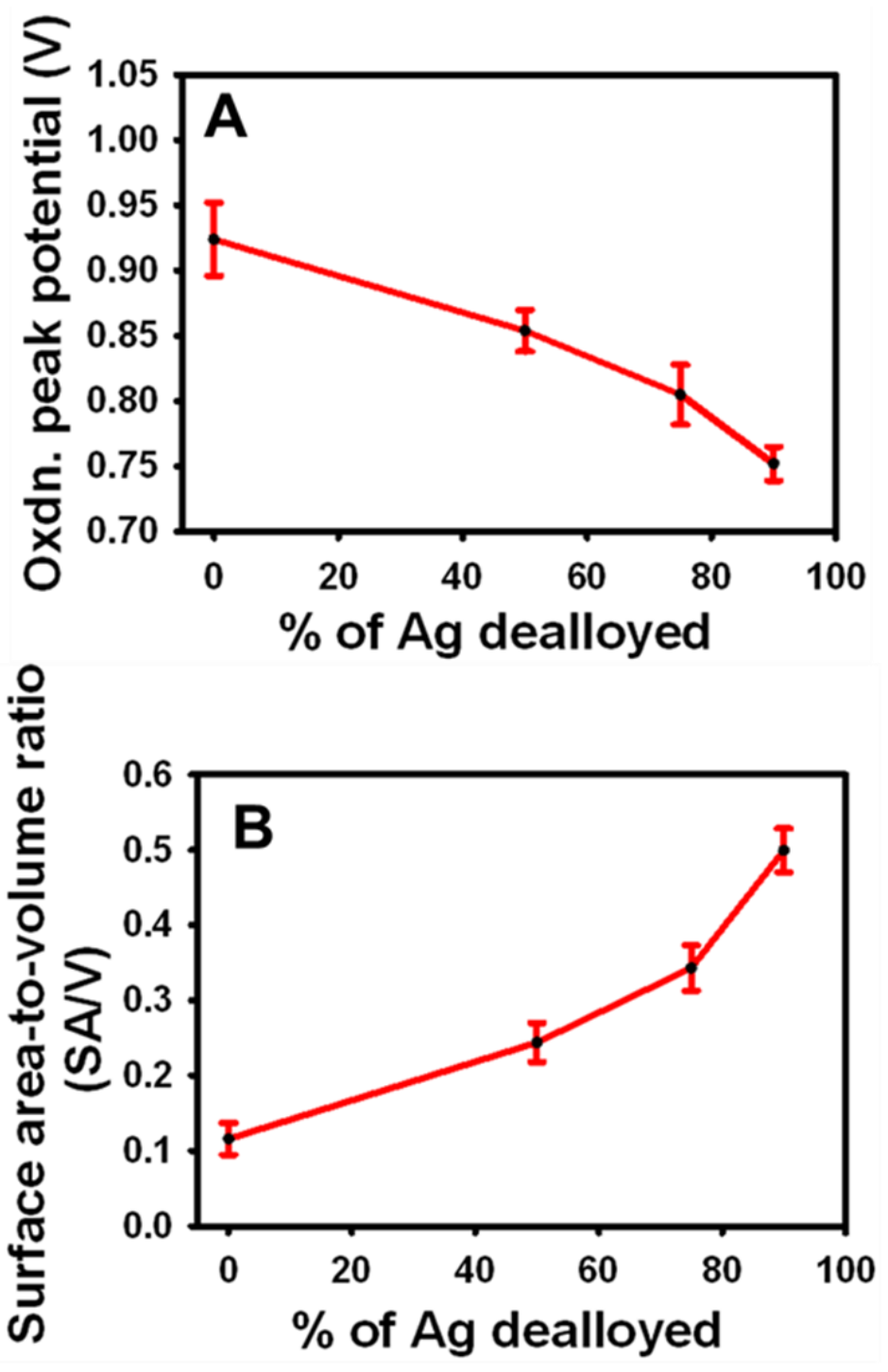

Figure 6. 8. Plots of $E_{p}(A)$ and $S A / V(B)$ vs vs $\%$ of $A g$ dealloyed from the different AuAg alloy NSs.

Based on these observations and to correlate the electrochemical SA/V of Au NSs obtained by dealloying to their $E_{p}$, we also plotted the $E_{p}$ as a function of SA/V 
(Figure 6.9). As can be seen from the plot, the $E_{p}$ decreased linearly with increasing SA/V, as observed for NSs assembled in different way in Chapter V. The trend of $E_{p}$ depending on the SA/V appears to hold for not only Au NSs of

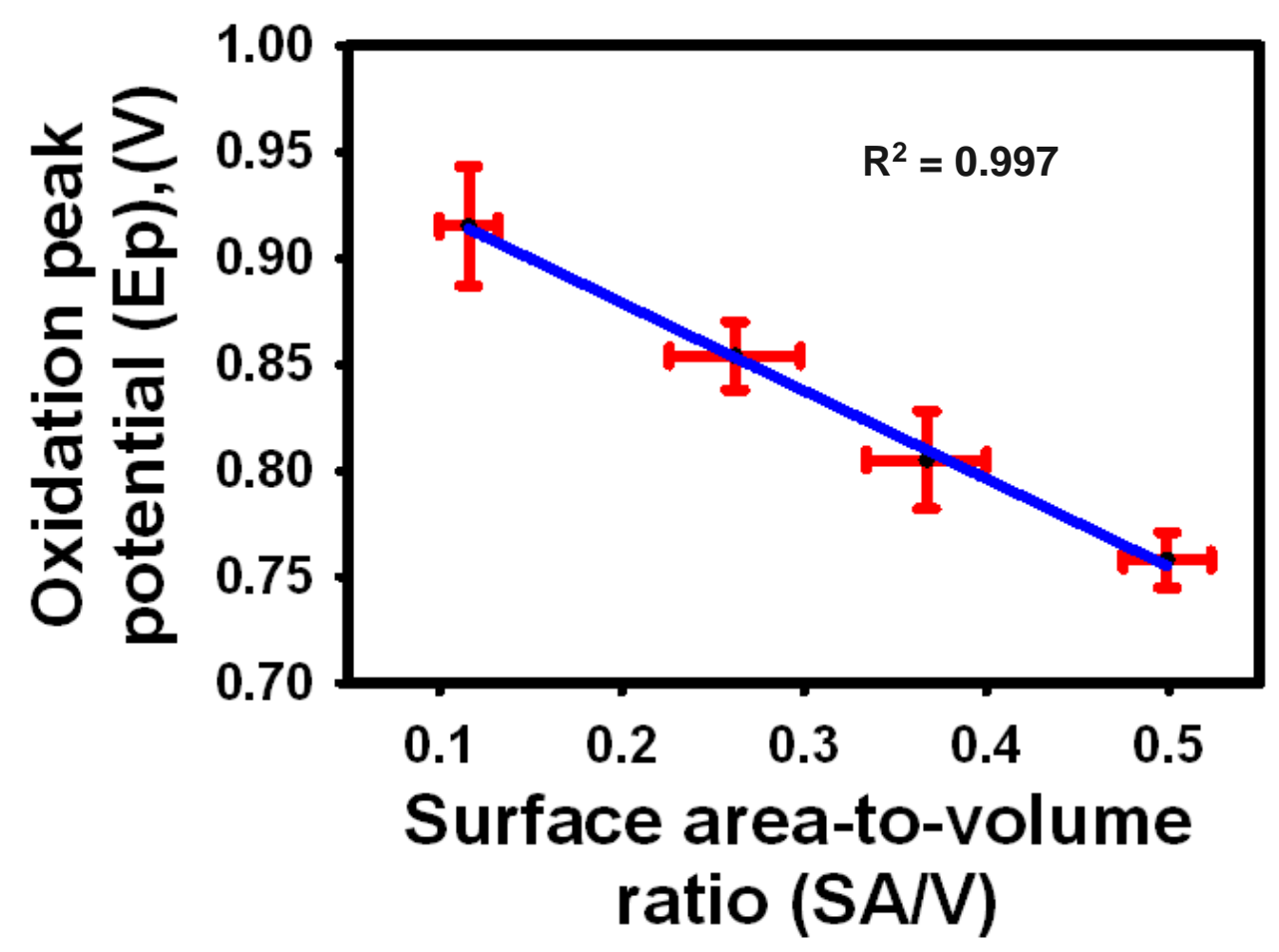

Figure 6. 9. Plot of $S A$-to-volume ratio $(S A / V)$ and oxidation peak potential (Ep) for Au NSs obtained by dealloying AuAg alloys having different percentatges of $\mathrm{Ag}$ in them

similar size whose SA/V is affected by their assembly method, but also for nanostructures that are irregularly shaped and do not have clearly defined dimensions. This is an important generalization that can be useful for 
understanding the oxidation properties of any shape nanostructures, including irregular-shaped, flattened, and porous nanomaterials.

\subsection{Conclusions}

In summary, we report an increase in SA/V and a negative thermodynamic shift in the oxidation potential $\left(E_{p}\right)$ for Au NSs obtained by dealloying $A_{450} A_{95}, A_{25} A_{75}$ and $\mathrm{Au}_{10} \mathrm{Ag}_{90}$ alloys compared to intact Au NSs of similar size (outer diameter). The $E_{p}$ decreased with increasing percentages of $\mathrm{Ag}$ dealloyed from the AuAg alloy NSs. Compared to the intact Au NSs having an average $E_{p}$ of $0.92 \mathrm{~V}$, the $\mathrm{Au}$ NSs obtained by dealloying of $A u_{50} A_{5}{ }_{50}, A_{25} A_{975}$ and $A_{10} A_{g} g_{90}$ showed $E_{p}$ values of $0.859 \mathrm{~V}, 0.805 \mathrm{~V}$ and $0.758 \mathrm{~V}$, respectively. The SA/ $\mathrm{increased}$ with increasing percentages of Ag dealloyed from the AuAg alloy NSs. Compared to the intact $\mathrm{Au}$ NSs having an average SA/V of 0.116 , the similar-sized Au NSs obtained by dealloying of Au50Ag50, Au25Ag75 and $A_{10} A_{10} A_{90}$ showed average SA/V of 0.262 , 0.343 and 0.499 , respectively. These trends for decreasing $E_{p}$ and increasing SA/V were due to the formation of distorted/flattened and porous Au nanostructures after dealloying the AuAg alloy NSs. This work is important in that it provides a simple electrochemical approach for measuring the SA/V of porous NSs, which can be useful for characterizing different synthetic methods and correlating the porosity to the activity, oxidative stability, and size stability. These are important issues for application of porous nanostructures in catalysis, sensing, and energy applications. 


\section{CHAPTER VII \\ SUMMARY, CONCLUSIONS AND FUTURE DIRECTIONS}

\subsection{Summary and Main Conclusions}

This dissertation describes the development of electrochemical measurements of the SA-to-volume ratio (SA/V) of metal NPs (NPs), with the focus on Au nanospheres (NSs), and the use of the SA/V measurement to analyze size, aggregation and porosity of metal NSs. In addition to the useful analysis, our work also provides new physical insight into what controls the thermodynamics of metal oxidation. This work therefore has importance both in the analysis and physical properties of metal nanostructures.

We demonstrated in Chapter III that the measured SA/V correlates well with the average radius of the Au NSs in a mathematically predictable way. Five different Au NSs with average radii of about $4 \mathrm{~nm}, 15 \mathrm{~nm}, 32 \mathrm{~nm}, 50 \mathrm{~nm}$ and 70 $\mathrm{nm}$ were synthesized and attached to glass/ITO/APTES electrodes. Measuring the number of coulombs of charge passed during the reduction of surface $\mathrm{Au}_{2} \mathrm{O}_{3}$ that was electrochemically-formed during $\mathrm{Au} \mathrm{NS}$ oxidation in $\mathrm{HClO}_{4}$ provided the $\mathrm{SA}$ (SA) while measuring the coulombs of charge passed during the complete oxidative dissolution of all of the Au in the Au NSs in the presence of $\mathrm{Br}^{-}$to form aqueous soluble $\mathrm{AuBr}_{4}{ }^{-}$gave the total volume (V). Assuming a spherical geometry, the $\mathrm{SA} / \mathrm{V}$ is theoretically equal to 3 divided by the average radius. A plot of electrochemically-meausured SA/V versus $1 /$ radius determined by scanning 
electron microscopy (SEM) for the 5 different sizes of Au NSs attached to the electrode was found to be linear with a slope of 1.8 without cleaning the electrode beyond simple rinsing and 3.5 when cleaning the surface with ozone treatment. This revealed that the SA measurement is not accurate without ozone cleaning, likely due to the presence of citrate stabilizer adsorbed to the Au NS surface prior to ozone cleaning. The main conclusion of this work was that the electrochemical measurement of SA/V is a simple, cost effective analytical tool to measure the size of metal NSs.

In Chapter IV we showed that the electrochemically-measured SA/V is sensitive to the aggregation state of $\mathrm{Au}$ metal NSs. The SA/V decreased for aggregated $4 \mathrm{~nm}$ and $15 \mathrm{~nm}$ Au NSs compared to their isolated forms and the peak oxidation potential $\left(E_{p}\right)$ of the aggregated Au NSs shifted positive in anodic stripping voltammetry (ASV). The main conclusions of this work are that aggregation leads to a decrease in SA/V and that the magnitude of the SA/V determines the $E_{p}$ value for metal NPs, where the $E_{p}$ increases as the SA/V decreases upon aggregation.

In Chapters V and VI, we described in more detail the effect of SA/V of $\mathrm{Au}$ NPs on their $E_{p}$ value in ASV. The general conclusion is that the SA/V of metal NPs is a better predictor of the $E_{p}$ value than the size of the metal NPs. Experiments confirmed that the $E_{p}$ value can be very different for the same-sized Au nanostructures if the SA/V is very different. In the first example, the $E_{p}$ of $A u$ NSs in ASV depended on how they were assembled onto glass/ITO electrodes (Chapter V). The different assembly methods included electrostatic attachment to 
an amine-functionalized silane linker, electrophoretic deposition (EPD), direct drop-cast deposition, and drop-cast deposition after mixing with carbon black. The same-sized Au NSs exhibited different $E_{p}$ values because the different assembly methods led to different SA/V values. The SA/V decreased in the order of linker > EPD > drop-cast deposition with carbon black > drop-cast deposition, while the $E_{p}$ values increased in that order. This clearly demonstrated the dependence of the SA/V of Au NSs on their thermodynamic oxidation potential. Another example of this occurred in the case of porous Au NSs formed by dealloying Ag from AuAg alloys (Chapter VI). In this case the NSs had similar outer diameters but very different SA/V due to differences in porosity and morphology. . The SA/V increased as the amount of $\mathrm{Ag}$ increased in the AuAg alloy before dealloying the $\mathrm{Ag}$ in the order of $\mathrm{Au}_{10} \mathrm{Ag}_{90}>\mathrm{Au}_{25} \mathrm{Ag}_{75}>\mathrm{Au}_{50} \mathrm{Ag}_{50}$ alloys. After dealloying, all of the NSs had a similar outer diameter of $\sim 50 \mathrm{~nm}$ but very different SA/V due to the formation of porous and distorted and flattened structures upon removing $\mathrm{Ag}$ from the alloy. The structures with higher SA/V had smaller $E_{p}$ values, again confirming that SA/V is a better predictor of $E_{p}$ than the size. This is an important general observation that provides a better understanding of what controls the thermodynamics of metal NP oxidation. after dealloying.

\subsection{Future Directions}

All of the work in this dissertation describes the electrochemical analysis of Au nanostructures. In the future, it will be very important to demonstrate these techniques for the analysis of other metals that are of technological importance, 
especially in catalysis and sensing applications. Other important metals include $\mathrm{Ag}, \mathrm{Cu}, \mathrm{Pt}$, and Pd. Similar to the Au metal NSs in this work, the electrochemical measurement of SA/V would be useful for the analysis of their size by a simple, convenient and cost-effective method. Different electrochemical approaches will be needed for the SA measurement and $\mathrm{V}$ measurement by stripping for each metal. There are reports available on the measurement of electrochemical SA of some of these other metals by underpotential deposition (UPD). The combination of UPD with stripping can provide the SA/V and NP size.

For work on the SA/V of aggregated forms of Au NSs, future work would include measuring the SA/V of more controlled aggregates, such as dimers and trimers and other well-defined superstructures. It will be interesting to determine how the SA/V will vary for the different types of aggregates and how that will impact the oxidation properties. It would also be interesting to determine if the $S A / V$ or $E_{p}$ depends on the spacing between the NSs after aggregation. Controlled spacing of different sizes of Au NSs, such as $4 \mathrm{~nm}$ and $15 \mathrm{~nm}$ NSs, can be synthesized using diamino linkers, such as 1,4 diaminobutane, 1,8 diaminooctane and 1,12 diaminododacane. These linkers electrostatically cross-link citrate-coated $4 \mathrm{~nm}$ and $15 \mathrm{~nm}$ Au NSs, leading to aggregation. Control over aggregate size could be obtained by adjusting the amount of the diamino linker. Aggregation studies can also be extended to $\mathrm{Ag}$ and Pd NPs.

The impact of the different assembly methods on the SA/V and oxidation properties of same-sized Au NSs can be extended to more relevant electrode materials than glass/ITO such as glassy carbon electrode, Pt disk electrodes, Au 
microelectrodes, and other types of carbon electrodes. These studies would be very valuable in understanding how SA/V of the metal nanostructures changes with the different electrodes and how that impacts their electrochemical activity for catalysis and sensing applications and their stability against oxidation and size transformations, such as Ostwald ripening.

Future studies on dealloying AuAg alloys with different Ag content can be extended to trying different concentrations of $\mathrm{Fe}\left(\mathrm{NO}_{3}\right)_{3} \cdot 9 \mathrm{H}_{2} \mathrm{O}$ and performing the dealloying treatment for different times. The effect of dealloying parameters on the final structure, and therefore the SA/V and $E_{p}$ values, would be very interesting. For the current conditions of dealloying we observed distorted and flattened $\mathrm{Au}$ NSs having increased SAs. It is possible that other dealloying conditions could lead to other, even more porous structures. We would try lower concentrations of $\mathrm{Fe}\left(\mathrm{NO}_{3}\right)_{3} \cdot 9 \mathrm{H}_{2} \mathrm{O}$ for longer duration (50 mM for 8 hours, for example) and higher concentrations of $\mathrm{Fe}\left(\mathrm{NO}_{3}\right)_{3} \cdot 9 \mathrm{H}_{2} \mathrm{O}$ for shorter duration $(400 \mathrm{mM}$ or $600 \mathrm{mM}$ for 30 minutes, for example). It would be interesting to observe the effect of these variations on the $S A$ and $E_{p}$ as related to the morphologies of the nanostructures formed. Another variation to try in the future would be to deposit the AuAg alloys on glass/ITO by EPD followed by dealloying using $\mathrm{Fe}\left(\mathrm{NO}_{3}\right)_{3} \cdot 9 \mathrm{H}_{2} \mathrm{O}$. As the NPs are distributed more evenly in the case of EPD, the nanostructures formed after dealloying can lead to the formation of smooth continuous porous films useful for different catalysis and sensing applications. The various parameters of EPD to be varied include deposition time and deposition potential. We would also try different alloy systems, such as AuCu alloys and different dealloying agents, such as $\mathrm{H}_{2} \mathrm{O}_{2}$ 
and $\mathrm{NH}_{4} \mathrm{OH}$ for both alloy systems. A great strength of the electrochemical analysis method is that it can be an excellent screening tool to study the properties of the metal nanostructures without having to characterize every sample by electron microscopy. If the results are promising as determined by electrochemistry first, then electron microscopy would be performed. This can save a great deal of time in nanomaterials synthesis and development. 


\section{REFERENCES}

1. Larmier, K.; Liao, W.-C.; Tada, S.; Lam, E.; Verel, R.; Bansode, A.; Urakawa, A.; Comas-Vives, A.; Copéret, C., CO2-to-Methanol Hydrogenation on Zirconia-Supported Copper Nanoparticles: Reaction Intermediates and the Role of the Metal-Support Interface. Angew. Chem. Int. Ed. 2017, 56, 2318-2323.

2. Tsumori, N.; Chen, L.; Wang, Q.; Zhu, Q.-L.; Kitta, M.; Xu, Q., Quasi-MOF: Exposing Inorganic Nodes to Guest Metal Nanoparticles for Drastically Enhanced Catalytic Activity. Chem 2018, 4, 845-856.

3. Hona, R. K.; Ramezanipour, F., Remarkable Oxygen-Evolution Activity of a Perovskite Oxide from the Ca2-xSrxFe2O6-ס Series. Angew. Chem. Int. Ed. Engl. 2019, 58, 2060-2063.

4. Hona, R. K.; Ramezanipour, F., Structure-dependence of electrical conductivity and electrocatalytic properties of Sr2Mn2O6 and CaSrMn2O6. J. Chem. Sci. 2019, 131, 109.

5. $\quad$ Ding, Y.; Yang, B.; Liu, H.; Liu, Z.; Zhang, X.; Zheng, X.; Liu, Q., Fept-Au Ternary Metallic Nanoparticles with the Enhanced Peroxidase-Like Activity for Ultrafast Colorimetric Detection of $\mathrm{H}_{2} \mathrm{O}_{2}$. Sens. Actuator B-Chem. 2018, 259, 775-783.

6. Jia, S.; Bian, C.; Sun, J.; Tong, J.; Xia, S., A Wavelength-Modulated Localized Surface Plasmon Resonance (Lspr) Optical Fiber Sensor for Sensitive Detection of Mercury(li) Ion by Gold Nanoparticles-DNA Conjugates. Biosens. Bioelectron. 2018, 114, $15-21$.

7. Raeesi, V.; Chou, L. Y. T.; Chan, W. C. W., Tuning the Drug Loading and Release of DNA-Assembled Gold-Nanorod Superstructures. Adv. Mater. 2016, 28, 8511-8518.

8. Zhu, J.; Wang, G.; Alves, C. S.; Tomás, H.; Xiong, Z.; Shen, M.; Rodrigues, J.; Shi, X., Multifunctional Dendrimer-Entrapped Gold Nanoparticles Conjugated with Doxorubicin for $\mathrm{pH}$-Responsive Drug Delivery and Targeted Computed Tomography Imaging. Langmuir 2018, 34, 12428-12435.

9. Xiao, P.; Bu, F.; Zhao, R.; Aly Aboud, M. F.; Shakir, I.; Xu, Y., Sub-5 nm Ultrasmall Metal-Organic Framework Nanocrystals for Highly Efficient Electrochemical Energy Storage. ACS Nano 2018, 12, 3947-3953.

10. Wang, L.; Huang, X.; Zhu, Y.; Jiang, P., Enhancing Electrical Energy Storage Capability of Dielectric Polymer Nanocomposites via the Room Temperature Coulomb Blockade Effect of Ultra-Small Platinum Nanoparticles. Phys. Chem. Chem. Phys. 2018, 20, 5001-5011.

11. Kharel, P.; Zamborini, F.; Alphenaar, B., Enhancing the Photovoltaic Performance of Dye-Sensitized Solar Cells with Rare-Earth Metal Oxide Nanoparticles. J. Electrochem. Soc. 2018, 165, H52-H56.

12. Kharel, P.; M Cuillier, P.; Fernando, K.; Zamborini, F.; Alphenaar, B., Effect of Rare-Earth Metal Oxide Nanoparticles on the Conductivity of Nanocrystalline Titanium Dioxide: An Electrical and Electrochemical Approach. J. Phys. Chem. C 2018, 122, 1509015096.

13. Dharmasena, R.; Thapa, A. K.; Hona, R. K.; Jasinski, J.; Sunkara, M. K.; Sumanasekera, G. U., Mesoporous TiO2 coating on carbon-sulfur cathode for high capacity Li-sulfur battery. RSC Adv. 2018, 8, 11622-11632.

14. Hona, R. K.; Ramezanipour, F., Enhanced electrical properties in BaSrFe2O6- $\delta$ $(\delta=0.5)$ : A disordered defect-perovskite. Polyhedron 2019, 167, 69-74. 
15. Back, S.; Yeom, M. S.; Jung, Y., Active Sites of Au and Ag Nanoparticle Catalysts for $\mathrm{CO}_{2}$ Electroreduction to CO. ACS Catal. 2015, 5, 5089-5096.

16. Hejral, U.; Franz, D.; Volkov, S.; Francoual, S.; Strempfer, J.; Stierle, A., Identification of a Catalytically Highly Active Surface Phase for CO Oxidation over PtRh Nanoparticles under Operando Reaction Conditions. Phys. Rev. Lett. 2018, 120, 126101. 17. Sil, D.; Hines, J.; Udeoyo, U.; Borguet, E., Palladium Nanoparticle-Based Surface Acoustic Wave Hydrogen Sensor. ACS Appl. Mater. Interfaces 2015, 7, 5709-5714.

18. Huang, Y.; Gao, L.; Cui, H., Assembly of Multifunctionalized Gold Nanoparticles with Chemiluminescent, Catalytic, and Immune Activity for Label-Free Immunoassays. ACS Appl. Mater. Interfaces 2018, 10, 17040-17046.

19. Mistry, H.; Reske, R.; Zeng, Z.; Zhao, Z.-J.; Greeley, J.; Strasser, P.; Cuenya, B. R., Exceptional Size-Dependent Activity Enhancement in the Electroreduction of $\mathrm{CO}_{2}$ over Au Nanoparticles. J. Am. Chem. Soc. 2014, 136, $16473-16476$.

20. Zhao, M.; Yuan, K.; Wang, Y.; Li, G.; Guo, J.; Gu, L.; Hu, W.; Zhao, H.; Tang, Z., Metal-Organic Frameworks as Selectivity Regulators for Hydrogenation Reactions. Nature 2016, 539, 76.

21. Baciu, C. L.; Becker, J.; Janshoff, A.; Sönnichsen, C., Protein-Membrane Interaction Probed by Single Plasmonic Nanoparticles. Nano Lett. 2008, 8, 1724-1728.

22. Ali, H. R.; Ali, M. R. K.; Wu, Y.; Selim, S. A.; Abdelaal, H. F. M.; Nasr, E. A.; ElSayed, M. A., Gold Nanorods as Drug Delivery Vehicles for Rifampicin Greatly Improve the Efficacy of Combating Mycobacterium tuberculosis with Good Biocompatibility with the Host Cells. Bioconjugate Chem. 2016, 27, 2486-2492.

23. Amjadi, M.; Hallaj, T.; Salari, R., A Highly Sensitive Plasmonic Sensor for Detection of Selenium Based on the Shape Transformation of Silver Nanoprisms. Sens. Actuator BChem. 2018, 273, 1307-1312.

24. Kilic, H.; Turgut, M.; Yilmaz, M. S.; Dalkilic, O.; Metin, Ö., Monodisperse Ni@Pd Core@Shell Nanoparticles Assembled on Reduced Graphene Oxide as a Highly Efficient and Reusable Heterogeneous Catalyst for the C-H Bond Arylation of Imidazo[1,2a]pyridine with Aryl Halides. ACS Sustain Chem Eng 2018, 6, 11433-11440.

25. Feng, Y.; Li, Z.; Liu, H.; Dong, C.; Wang, J.; Kulinich, S. A.; Du, X., Laser-Prepared CuZn Alloy Catalyst for Selective Electrochemical Reduction of CO2 to Ethylene. Langmuir 2018, 34, 13544-13549.

26. Veisi, H.; Pirhayati, M.; Kakanejadifard, A.; Mohammadi, P.; Abdi, M. R.; Gholami, J.; Hemmati, S., In Situ Green Synthesis of Pd Nanoparticles on Tannic Acid-Modified Magnetite Nanoparticles as a Green Reductant and Stabilizer Agent: Its Application as a Recyclable Nanocatalyst (Fe3O4@ TA/Pd) for Reduction of 4-Nitrophenol and Suzuki Reactions. ChemistrySelect 2018, 3, 1820-1826.

27. Kanso, H.; Pankratova, G.; Bollella, P.; Leech, D.; Hernandez, D.; Gorton, L., Sunlight photocurrent generation from thylakoid membranes on gold nanoparticle modified screen-printed electrodes. J. Electroanal. Chem. 2018, 816, 259-264.

28. Yang, B.; Chou, J.; Dong, X.; Qu, C.; Yu, Q.; Lee, K. J.; Harvey, N., Size-Controlled Green Synthesis of Highly Stable and Uniform Small to Ultrasmall Gold Nanoparticles by Controlling Reaction Steps and pH. The Journal of Physical Chemistry C 2017, 121 (16), 8961-8967.

29. Mogensen, K. B.; Kneipp, K., Size-Dependent Shifts of Plasmon Resonance in Silver Nanoparticle Films Using Controlled Dissolution: Monitoring the Onset of Surface Screening Effects. J. Phys. Chem. C 2014, 118, 28075-28083.

30. Peretyazhko, T. S.; Zhang, Q.; Colvin, V. L., Size-Controlled Dissolution of Silver Nanoparticles at Neutral and Acidic pH Conditions: Kinetics and Size Changes. Environ. Sci. Technol. 2014, 48, 11954-11961. 
31. Qian, K.; Sweeny, B. C.; Johnston-Peck, A. C.; Niu, W.; Graham, J. O.; DuChene, J. S.; Qiu, J.; Wang, Y.-C.; Engelhard, M. H.; Su, D.; Stach, E. A.; Wei, W. D., Surface Plasmon-Driven Water Reduction: Gold Nanoparticle Size Matters. J. Am. Chem. Soc. 2014, 136, 9842-9845.

32. Li, X.; Chen, Q.; McCue, I.; Snyder, J.; Crozier, P.; Erlebacher, J.; Sieradzki, K., Dealloying of Noble-Metal Alloy Nanoparticles. Nano Lett. 2014, 14, $2569-2577$.

33. Jara, D. H.; Yoon, S. J.; Stamplecoskie, K. G.; Kamat, P. V., Size-Dependent Photovoltaic Performance of CulnS2 Quantum Dot-Sensitized Solar Cells. Chem. Mater. 2014, 26, 7221-7228.

34. Carroll, I.; Klowden, T.; Alvarez, I.; Postma, H. W. C., Characterization of Au catalytic activity in low-temperature graphene etching. Surf. Sci. 2019, 688, 25-30.

35. Hosseini, S.; Alsiraey, N.; Riley, A. J.; Zubkov, T.; Closson, T.; Tye, J.; Bodappa, N.; Li, Z., Variable Growth and Characterizations of Monolayer-Protected Gold Nanoparticles Based on Molar Ratio of Gold and Capping Ligands. Langmuir 2018, 34 (50), 15517-15525.

36. Nguyen, H. A.; Banerjee, P.; Nguyen, D.; Lyding, J. W.; Gruebele, M.; Jain, P. K., STM Imaging of Localized Surface Plasmons on Individual Gold Nanoislands. The Journal of Physical Chemistry Letters 2018, 9 (8), 1970-1976.

37. Najafi, M.; Di Giacomo, F.; Zhang, D.; Shanmugam, S.; Senes, A.; Verhees, W.; Hadipour, A.; Galagan, Y.; Aernouts, T.; Veenstra, S.; Andriessen, R., Highly Efficient and Stable Flexible Perovskite Solar Cells with Metal Oxides Nanoparticle Charge Extraction Layers. Small 2018, 14, 1702775.

38. Deng, X.; Galli, F.; Koper, M. T. M., In Situ Electrochemical AFM Imaging of a Pt Electrode in Sulfuric Acid under Potential Cycling Conditions. J. Am. Chem. Soc. 2018, 140, 13285-13291.

39. Wibroe, P. P.; Anselmo, A. C.; Nilsson, P. H.; Sarode, A.; Gupta, V.; Urbanics, R.; Szebeni, J.; Hunter, A. C.; Mitragotri, S.; Mollnes, T. E.; Moghimi, S. M., Bypassing Adverse Injection Reactions to Nanoparticles Through Shape Modification and Attachment to Erythrocytes. Nat. Nanotechnol 2017, 12, 589.

40. Dias, C. S. B.; Hanchuk, T. D. M.; Wender, H.; Shigeyosi, W. T.; Kobarg, J.; Rossi, A. L.; Tanaka, M. N.; Cardoso, M. B.; Garcia, F., Shape Tailored Magnetic Nanorings for Intracellular Hyperthermia Cancer Therapy. Sci Rep 2017, 7, 14843.

41. Lin, K.-Q.; Yi, J.; Hu, S.; Liu, B.-J.; Liu, J.-Y.; Wang, X.; Ren, B., Size Effect on SERS of Gold Nanorods Demonstrated via Single Nanoparticle Spectroscopy. J. Phys. Chem. C 2016, 120, 20806-20813.

42. Masitas, R. A.; Allen, S. L.; Zamborini, F. P., Size-Dependent Electrophoretic Deposition of Catalytic Gold Nanoparticles. J. Phys. Chem. C 2016, 138, 15295-15298.

43. Allen, S. L.; Sharma, J. N.; Zamborini, F. P., Aggregation-Dependent Oxidation of Metal Nanoparticles. J. Am. Chem. Soc. 2017, 139, 12895-12898.

44. Kawamoto, N.; Tang, D. M.; Wei, X.; Wang, X.; Mitome, M.; Bando, Y.; Golberg, D., Transmission Electron Microscope as an Ultimate Tool for Nanomaterial Property Studies. Microscopy (Oxford, England) 2013, 62, 157-175.

45. Yang, W.; Liu, X.; Yue, X.; Jia, J.; Guo, S., Bamboo-like Carbon Nanotube/Fe3C Nanoparticle Hybrids and Their Highly Efficient Catalysis for Oxygen Reduction. J. Phys. Chem. C 2015, 137, 1436-1439.

46. Zhang, S.; Katz, M. B.; Dai, S.; Zhang, K.; Du, X.; Graham, G. W.; Pan, X., New Atomic-Scale Insight into Self-Regeneration of Pt-CaTiO3 Catalysts: Incipient RedoxInduced Structures Revealed by a Small-Angle Tilting STEM Technique. J. Phys. Chem. C 2017, 121, 17348-17353. 
47. Bastús, N. G.; Comenge, J.; Puntes, V., Kinetically Controlled Seeded Growth Synthesis of Citrate-Stabilized Gold Nanoparticles of up to $200 \mathrm{~nm}$ : Size Focusing versus Ostwald Ripening. Langmuir 2011, 27, 11098-11105.

48. Juvé, V.; Cardinal, M. F.; Lombardi, A.; Crut, A.; Maioli, P.; Pérez-Juste, J.; LizMarzán, L. M.; Del Fatti, N.; Vallée, F., Size-Dependent Surface Plasmon Resonance Broadening in Nonspherical Nanoparticles: Single Gold Nanorods. Nano Lett. 2013, 13, 2234-2240.

49. Balog, S.; Rodriguez-Lorenzo, L.; Monnier, C. A.; Obiols-Rabasa, M.; RothenRutishauser, B.; Schurtenberger, P.; Petri-Fink, A., Characterizing Nanoparticles in Complex Biological Media and Physiological Fluids wth Depolarized Dynamic Light Scattering. Nanoscale 2015, 7 (14), 5991-5997.

50. Yu, L.; You, H.; Zhang, Q.; Zhang, L.; Fang, J., Digestive Ripening Mechanism Investigation in A Classical Lee-Meisel Method Based on In Situ UV-Vis Spectra. CrystEngComm 2019, 21, 1529-1533

51. Agrawal, A.; Kriegel, I.; Milliron, D. J., Shape-Dependent Field Enhancement and Plasmon Resonance of Oxide Nanocrystals. J. Phys. Chem. C 2015, 119, 6227-6238.

52. Zook, J. M.; Rastogi, V.; Maccuspie, R. I.; Keene, A. M.; Fagan, J., Measuring Agglomerate Size Distribution and Dependence of Localized Surface Plasmon Resonance Absorbance on Gold Nanoparticle Agglomerate Size Using Analytical Ultracentrifugation. ACS Nano 2011, 5, 8070-9.

53. Stetefeld, J.; McKenna, S. A.; Patel, T. R., Dynamic Light Scattering: A Practical Guide and Applications in Biomedical Sciences. Biophys. Rev. 2016, 8, 409-427.

54. Zheng, T.; Bott, S.; Huo, Q., Techniques for Accurate Sizing of Gold Nanoparticles Using Dynamic Light Scattering with Particular Application to Chemical and Biological Sensing Based on Aggregate Formation. ACS Appl. Mater. Interfaces 2016, 8, 2158521594.

55. Jans, H.; Liu, X.; Austin, L.; Maes, G.; Huo, Q., Dynamic Light Scattering as a Powerful Tool for Gold Nanoparticle Bioconjugation and Biomolecular Binding Studies. Analytical Chemistry 2009, 81 (22), 9425-9432.

56. Castaneda, A. D.; Robinson, D. A.; Stevenson, K. J.; Crooks, R. M., Electrocatalytic Amplification of DNA-Modified Nanoparticle Collisions via Enzymatic Digestion. Chem. Sci. 2016, 7, 6450-6457.

57. Kang, M.; Perry, D.; Kim, Y. R.; Colburn, A. W.; Lazenby, R. A.; Unwin, P. R., TimeResolved Detection and Analysis of Single Nanoparticle Electrocatalytic Impacts. J. Am. Chem. Soc. 2015, 137, 10902-5.

58. Chaudhari, V. R.; Hassan, P. A.; Haram, S. K., Size-Dependent Quantized Double Layer Charging of Monolayer-Protected Silver Nanoparticles. New J Chem 2014, 38, 1761-1767.

59. Barisik, M.; Atalay, S.; Beskok, A.; Qian, S., Size Dependent Surface Charge Properties of Silica Nanoparticles. J. Phys. Chem. C 2014, 118, 1836-1842.

60. Goyal, G.; Freedman, K. J.; Kim, M. J., Gold Nanoparticle Translocation Dynamics and Electrical Detection of Single Particle Diffusion Using Solid-State Nanopores. Anal. Chem. 2013, 85, 8180-8187.

61. Rudzevich, Y.; Lin, Y.; Wearne, A.; Ordonez, A.; Lupan, O.; Chow, L., Characterization of Liposomes and Silica Nanoparticles Using Resistive Pulse Method. Colloids Surf., A 2014, 448, 9-15.

62. Battistel, D.; Baldi, F.; Gallo, M.; Faleri, C.; Daniele, S., Characterisation of Biosynthesised Silver Nanoparticles by Scanning Electrochemical Microscopy (SECM) and Voltammetry. Talanta 2015, 132, 294-300. 
63. Kim, J.; Renault, C.; Nioradze, N.; Arroyo-Currás, N.; Leonard, K. C.; Bard, A. J., Electrocatalytic Activity of Individual Pt Nanoparticles Studied by Nanoscale Scanning Electrochemical Microscopy. J. Am. Chem. Soc. 2016, 138, 8560-8568.

64. Huerta, T. F.; Valenzuela, J., Growth of 4-Aminothiophenol on lodine Modified $\mathrm{Au}(100)$ Studied by Scanning Tunneling Microscopy. Surf. Sci. 2017, 655, 17-24.

65. Zamborini, F. P.; Crooks, R. M., In-Situ Electrochemical Scanning Tunneling Microscopy (ECSTM) Study of Cyanide-Induced Corrosion of Naked and Hexadecyl Mercaptan-Passivated Au(111). Langmuir 1997, 13, 122-126.

66. Snowden, M. E.; Güell, A. G.; Lai, S. C. S.; McKelvey, K.; Ebejer, N.; O'Connell, M. A.; Colburn, A. W.; Unwin, P. R., Scanning Electrochemical Cell Microscopy: Theory and Experiment for Quantitative High Resolution Spatially-Resolved Voltammetry and Simultaneous lon-Conductance Measurements. Anal. Chem. 2012, 84, 2483-2491.

67. E, S. P.; Kim, Y.-R.; Perry, D.; Bentley, C. L.; Unwin, P. R., Nanoscale Electrocatalysis of Hydrazine Electro-Oxidation at Blistered Graphite Electrodes. ACS Appl. Mater. Interfaces 2016, 8, 30458-30466.

68. Toma, F. M.; Cooper, J. K.; Kunzelmann, V.; McDowell, M. T.; Yu, J.; Larson, D. M.; Borys, N. J.; Abelyan, C.; Beeman, J. W.; Yu, K. M.; Yang, J.; Chen, L.; Shaner, M. R.; Spurgeon, J.; Houle, F. A.; Persson, K. A.; Sharp, I. D., Mechanistic Insights into Chemical and Photochemical Transformations of Bismuth Vanadate Photoanodes. Nat. Commun 2016, 7, 12012.

69. Koinuma, M.; Uosaki, K., An Electrochemical AFM Study on Electrodeposition of Copper on p-GaAs( (100) Surface in HCl Solution. Electrochim. Acta 1995, 40, 1345-1351.

70. $\quad$ Kang, M.; Perry, D.; Bentley, C. L.; West, G.; Page, A.; Unwin, P. R., Simultaneous Topography and Reaction Flux Mapping at and around Electrocatalytic Nanoparticles. ACS Nano 2017, 11, 9525-9535.

71. Novak, P.; Shevchuk, A.; Ruenraroengsak, P.; Miragoli, M.; Thorley, A. J.; Klenerman, D.; Lab, M. J.; Tetley, T. D.; Gorelik, J.; Korchev, Y. E., Imaging Single Nanoparticle Interactions with Human Lung Cells Using Fast Ion Conductance Microscopy. Nano Lett. 2014, 14, 1202-1207.

72. Sudha, V.; Sangaranarayanan, M. V., Underpotential Deposition of Metals: Structural and Thermodynamic Considerations. J. Phys. Chem. B 2002, 106, 2699-2707. 73. Watt-Smith, M. J.; Friedrich, J. M.; Rigby, S. P.; Ralph, T. R.; Walsh, F. C., Determination of the Electrochemically Active Surface Area of Pt/C PEM Fuel Cell Electrodes Using Different Adsorbates. J. Phys. D 2008, 41, 174004.

74. Swathirajan, S.; Bruckenstein, S., Thermodynamics and Kinetics of Underpotential Deposition of Metal Monolayers on Polycrystalline Substrates. Electrochim. Acta 1983, 28, 865-877.

75. Liu, Y.; Bliznakov, S.; Dimitrov, N., Comprehensive Study of the Application of a $\mathrm{Pb}$ Underpotential Deposition-Assisted Method for Surface Area Measurement of Metallic Nanoporous Materials. J. Am. Chem. Soc. 2009, 113, 12362-12372.

76. Green, C. L.; Kucernak, A., Determination of the Platinum and Ruthenium Surface Areas in Platinum-Ruthenium Alloy Electrocatalysts by Underpotential Deposition of Copper. I. Unsupported Catalysts. J. Phys. Chem. B 2002, 106, 1036-1047.

77. Wang, T. C.; Bury, W.; Gomez-Gualdron, D. A.; Vermeulen, N. A.; Mondloch, J. E.; Deria, P.; Zhang, K.; Moghadam, P. Z.; Sarjeant, A. A.; Snurr, R. Q.; Stoddart, J. F.; Hupp, J. T.; Farha, O. K., Ultrahigh Surface area Zirconium MOFs and Insights into the Applicability of the BET Theory. J Am Chem Soc 2015, 137, 3585-91.

78. Walton, K. S.; Snurr, R. Q., Applicability of the BET Method for Determining Surface Areas of Microporous Metal-Organic Frameworks. J Am Chem Soc 2007, 129, 8552-8556. 
79. Henglein, A., Physicochemical Properties of Small Metal Particlesin Solution: "Microelectrode" Reactions, Chemisorption, Composite Metal Particles, and The AtomTo-Metal Transition. J. Phys. Chem. 1993, 97, 5457-5471.

80. Plieth, W. J., Electrochemical Properties of Small Clustersof Metal Atoms and Their Role in the Surface Enhanced Raman Scattering. J. Phys. Chem. 1982, 86, 31663170.

81. Brainina, K. Z.; Galperin, L. G.; Galperin, A. L., Mathematical Modeling and Numerical Simulation of Metal Nanoparticles Electrooxidation. J. Solid State Electrochem. 2009, 14, 981-988.

82. Brainina, K. Z.; Galperin, L.; Vikulova, E., Electrochemistry of Metal Nanoparticles: The Effect of Substrate. J. Solid State Electrochem. 2012, 16, 2357-2363.

83. Tang, L.; Han, B.; Persson, K.; Friesen, C.; He, T.; Sieradzki, K.; Ceder, G., Electrochemical Stability of Nanometer-Scale Pt Particles in Acidic Environments. J. Am. Chem. Soc. 2010, 132, 596-600.

84. Kumar, A.; Buttry, D. A., Size-Dependent Anodic Dissolution of Water-Soluble Palladium Nanoparticles. J. Phys. Chem. C 2013, 117 (50), 26783-26789.

85. Ivanova, O. S.; Zamborini, F. P., Size-Dependent Electrochemical Oxidation of Silver Nanoparticles. J. Am. Chem. Soc. 2009, 132, 70-72.

86. Ivanova, O. S.; Zamborini, F. P., Electrochemical Size Discrimination of Gold Nanoparticles Attached to Glass/Indium- Tin-Oxide Electrodes by Oxidation In BromideContaining Electrolyte. Anal. Chem. 2010, 82, 5844-5850.

87. Sharma, J. N.; Pattadar, D. K.; Mainali, B. P.; Zamborini, F. P., Electrochemical determination of surface area-to-volume ratio for metal nanoparticle size analysis. Abstracts of Papers, 255th ACS National Meeting Exposition, New Orleans, LA, United States, March 18-22, 20182018.

88. Sharma, J. N.; Pattadar, D. K.; Mainali, B. P.; Zamborini, F. P., Size Determination of Metal Nanoparticles Based on Electrochemically Measured Surface-Area-to-Volume Ratios. Anal. Chem. 2018, 90, 9308-9314.

89. Kim, J.-H.; Lavin, B. W.; Boote, B. W.; Pham, J. A., Photothermally Enhanced Catalytic Activity of Partially Aggregated Gold Nanoparticles. J. Nanoparticle Res. 2012, 14, 1-10.

90. Martin, L. C.; Larmour, I. A.; Faulds, K.; Graham, D., Turning Up the LightsFabrication of Brighter SERRS Nanotags. Chem. Commun. 2010, 46, 5247-5249.

91. Zhang, J.; Fu, Y.; Chowdhury, M. H.; Lakowicz, J. R., Metal-Enhanced SingleMolecule Fluorescence on Silver Particle Monomer and Dimer: Coupling Effect between Metal Particles. Nano Lett. 2007, 7, 2101-2107.

92. Xu, L.; Kuang, H.; Xu, C.; Ma, W.; Wang, L.; Kotov, N. A., Regiospecific Plasmonic Assemblies for in Situ Raman Spectroscopy in Live Cells. J. Am. Chem. Soc. 2012, 134, 1699-1709.

93. Lin, V. K.; Teo, S. L.; Marty, R.; Arbouet, A.; Girard, C.; Alarcon-Llado, E.; Liu, S. H.; Han, M. Y.; Tripathy, S.; Mlayah, A., Dual Wavelength Sensing Based on Interacting Gold Nanodisk Trimers. Nanotechnology 2010, 21, 305501.

94. Bonham, A. J.; Braun, G.; Pavel, I.; Moskovits, M.; Reich, N. O., Detection of Sequence-Specific Protein-DNA Interactions via Surface Enhanced Resonance Raman Scattering. J. Am. Chem. Soc. 2007, 129, $14572-14573$.

95. Nambiathan Nambiar, H., Aggregation-dependent oxidation of different-sized gold nanoparticles. Abstracts, 70th Southeastern Regional Meeting of the American Chemical Society, Augusta, GA, United States, October 31-November 32018.

96. Sharma, J. N.; Pattadar, D. K.; Mainali, B. P.; Zamborini, F. P., Understanding the Role of Nanoparticle Assembly Method and Electrode Material on the Electrochemically- 
Determined Surface Area-To-Volume Ratio. Abstracts, 70th Southeastern Regional Meeting of the American Chemical Society, Augusta, GA, United States, October 31November 32018.

97. Masitas, R. A.; Khachian, I. V.; Bill, B. L.; Zamborini, F. P., Effect of Surface Charge and Electrode Material on the Size-Dependent Oxidation of Surface-Attached Metal Nanoparticles. Langmuir 2014, 30, 13075-13084.

98. Hu, X.-B.; Liu, Y.-L.; Zhang, H.-W.; Xiao, C.; Qin, Y.; Duo, H.-H.; Xu, J.-Q.; Guo, S.; Pang, D.-W.; Huang, W.-H., Electrochemical Monitoring of Hydrogen Sulfide Release from Single Cells. ChemElectroChem 2016, 3, 1998-2002.

99. Jia, F.; Yu, C.; Ai, Z.; Zhang, L., Fabrication of Nanoporous Gold Film Electrodes with Ultrahigh Surface Area and Electrochemical Activity. Chem. Mater. 2007, 19, 36483653.

100. Yang, S.; Zheng, Y.; Zhang, X.; Ding, S.; Li, L.; Zha, W., Molecularly Imprinted Electrochemical Sensor Based on the Synergic Effect of Nanoporous Gold and Copper Nanoparticles for the Determination of Cysteine. J. Solid State Electrochem. 2016, 20, 2037-2044.

101. Cherevko, S.; Chung, C.-H., Direct electrodeposition of nanoporous gold with controlled multimodal pore size distribution. Electrochem. Commun. 2011, 13, 16-19.

102. Cattarin, S.; Kramer, D.; Lui, A.; Musiani, M. M., Preparation and Characterization of Gold Nanostructures of Controlled Dimension by Electrochemical Techniques. J. Phys. Chem. C 2007, 111, 12643-12649.

103. McCurry, D. A.; Kamundi, M.; Fayette, M.; Wafula, F.; Dimitrov, N., All Electrochemical Fabrication of a Platinized Nanoporous Au Thin-Film Catalyst. ACS Appl. Mater. Interfaces 2011, 3, 4459-4468.

104. Snyder, J.; Livi, K.; Erlebacher, J., Dealloying Silver/Gold Alloys in Neutral Silver Nitrate Solution: Porosity Evolution, Surface Composition, and Surface Oxides. J. Electrochem. Soc. 2008, 155, C464-C473.

105. Zhang, Q.; Cobley, C. M.; Zeng, J.; Wen, L.-P.; Chen, J.; Xia, Y., Dissolving Ag from Au-Ag Alloy Nanoboxes with $\mathrm{H} 2 \mathrm{O} 2$ : A Method for Both Tailoring the Optical Properties and Measuring the H2O2 Concentration. J. Phys. Chem. C 2010, 114, 63966400.

106. Kim, M.; Ko, S. M.; Nam, J.-M., Dealloying-Based Facile Synthesis and Highly Catalytic Properties of Au Core/Porous Shell Nanoparticles. Nanoscale 2016, 8 (22), 11707-11717.

107. Vega, A. A.; Newman, R. C., Nanoporous Metals Fabricated Through Electrochemical Dealloying of $\mathrm{Ag}-\mathrm{Au}-\mathrm{Pt}$ with Systematic Variation of Au: Pt Ratio. J. Electrochem. Soc. 2014, 161, C1-C10.

108. Wang, D.; Zhao, P.; Li, Y., General Preparation for Pt-Based Alloy Nanoporous Nanoparticles as Potential Nanocatalysts. Sci. Rep. 2011, 1, 37.

109. Duff, D. G.; Baiker, A.; Edwards, P. P., A new hydrosol of gold clusters. 1. Formation and particle size variation. Langmuir 1993, 9, 2301-2309.

110. Jana, N. R.; Gearheart, L.; Murphy, C. J., Wet Chemical Synthesis of High Aspect Ratio Cylindrical Gold Nanorods. J. Phys. Chem. B 2001, 105, 4065-4067.

111. Turkevich, J.; Stevenson, P. C.; Hillier, J., A Study of the Nucleation and Growth Processes in the Synthesis of Colloidal Gold. Discuss. Faraday Soc. 1951, 11, 55-75.

112. Liu, X.; Xu, H.; Xia, H.; Wang, D., Rapid Seeded Growth of Monodisperse, QuasiSpherical, Citrate-Stabilized Gold Nanoparticles via H2o2 Reduction. Langmuir 2012, 28, 13720-13726.

113. Zamborini Francis, P., Analyzing size, composition, aggregation state, and electrophoretic deposition of metal and alloy nanoparticles by stripping voltammetry. 
Abstracts, 69th Southeastern Regional Meeting of the American Chemical Society, Charlotte, NC, United States, November 7-11 2017.

114. Mainali Badri, P., Sintering of metal nanoparticles through repetitive electrochemical oxidation-reduction cycles. Abstracts, 70th Southeastern Regional Meeting of the American Chemical Society, Augusta, GA, United States, October 31November 32018.

115. Zamborini Francis, P., Analyzing metal nanoparticle transformations by anodic stripping voltammetry. Abstracts of Papers, 257th ACS National Meeting Exposition, Orlando, FL, United States, Mar. 31-Apr. 4, 20192019.

116. Mainali Badri, P., Size-dependent oxidation behavior of gold nanoparticles coated with alkanethiol self-assembled monolayers (SAMs). Abstracts of Papers, 255th ACS National Meeting Exposition, New Orleans, LA, United States, March 18-22, 20182018.

117. Wu, B.; Zheng, N., Surface and Interface Control of Noble Metal Nanocrystals for Catalytic and Electrocatalytic Applications. Nano Today 2013, 8, 168-197.

118. Ibanez, F. J.; Zamborini, F. P., Chemiresistive Sensing with Chemically Modified Metal and Alloy Nanoparticles. Small 2012, 8, 174-202.

119. Gschneidtner, T. A.; Diaz Fernandez, Y. A.; Moth-Poulsen, K., Progress in SelfAssembled Single-Molecule Electronic Devices. J. Mater. Chem. C 2013, 1, 7127-7133

120. Biju, V., Chemical Modifications and Bioconjugate Reactions of Nanomaterials for Sensing, Imaging, Drug Delivery and Therapy. Chem. Soc. Rev. 2014, 43, 744-64.

121. Akhter, S.; Ahmad, M. Z.; Ahmad, F. J.; Storm, G.; Kok, R. J., Gold Nanoparticles in Theranostic Oncology: Current State-of-the-Art. Expert Opin. Drug Deliv. 2012, 9, 122543.

122. Murray, W. A.; Barnes, W. L., Plasmonic Materials. Adv. Mater. 2007, 19, 3771 3782.

123. Reske, R.; Mistry, H.; Behafarid, F.; Roldan Cuenya, B.; Strasser, P., Particle Size Effects in the Catalytic Electroreduction of $\mathrm{CO}_{2}$ on Cu Nanoparticles. J. Am. Chem. Soc. 2014, 136, 6978-6986.

124. Gao, D.; Zhou, H.; Wang, J.; Miao, S.; Yang, F.; Wang, G.; Wang, J.; Bao, X., SizeDependent Electrocatalytic Reduction Of $\mathrm{CO}_{2}$ Over Pd Nanoparticles. J. Am. Chem. Soc. 2015, 137, 4288-91.

125. Jin, R.; Sun, S.; Yang, Y.; Xing, Y.; Yu, D.; Yu, X.; Song, S., Size-Dependent Catalytic Properties of $\mathrm{Au}$ Nanoparticles Supported on Hierarchical Nickel Silicate Nanostructures. Dalton Trans. 2013, 42, 7888-7893.

126. Choi, O.; Hu, Z., Size Dependent and Reactive Oxygen Species Related Nanosilver Toxicity to Nitrifying Bacteria. Environ. Sci. Technol. 2008, 42, 4583-4588.

127. Haiss, W.; Thanh, N. T.; Aveyard, J.; Fernig, D. G., Determination of Size and Concentration of Gold Nanoparticles from Uv- Vis Spectra. Anal. Chem. 2007, 79 (11), 4215-4221.

128. Agnihotri, S.; Mukherji, S.; Mukherji, S., Size-Controlled Silver Nanoparticles Synthesized Over the Range 5-100 Nm Using the Same Protocol and Their Antibacterial Efficacy. RSC Adv. 2014, 4, 3974-3983.

129. Mistry, H.; Reske, R.; Strasser, P.; Cuenya, B. R., Size-dependent reactivity of gold-copper bimetallic nanoparticles during $\mathrm{CO} 2$ electroreduction. Catal. Today 2017, 288, 30-36.

130. Iqbal, M.; Usanase, G.; Oulmi, K.; Aberkane, F.; Bendaikha, T.; Fessi, H.; Zine, N.; Agusti, G.; Errachid, E.-S.; Elaissari, A., Preparation of Gold Nanoparticles and Determination of Their Particles Size via Different Methods. Mater. Res. Bull. 2016, 79, 97-104. 
131. Loget, G.; Lee, T. C.; Taylor, R. W.; Mahajan, S.; Nicoletti, O.; Jones, S. T.; Coulston, R. J.; Lapeyre, V.; Garrigue, P.; Midgley, P. A.; Scherman, O. A.; Baumberg, J. J.; Kuhn, A., Direct Visualization of Symmetry Breaking During Janus Nanoparticle Formation. Small 2012, (17), 2698-2703.

132. Klapetek, P.; Valtr, M.; Nečas, D.; Salyk, O.; Dzik, P., Atomic force microscopy analysis of nanoparticles in non-ideal conditions. Nanoscale Res. Lett. 2011, 6 (1), 514.

133. Filipe, V.; Hawe, A.; Jiskoot, W., Critical Evaluation of Nanoparticle Tracking Analysis (Nta) by Nanosight for The Measurement of Nanoparticles and Protein Aggregates. Pharm Res. 2010, 27, 796-810.

134. Zhou, Y. G.; Rees, N. V.; Pillay, J.; Tshikhudo, R.; Vilakazi, S.; Compton, R. G., Gold Nanoparticles Show Electroactivity: Counting and Sorting Nanoparticles Upon Impact with Electrodes. Chem. Commun. 2012, 48, 224-226.

135. Zhou, Y. G.; Rees, N. V.; Compton, R. G., The Electrochemical Detection and Characterization of Silver Nanoparticles in Aqueous Solution. Angew. Chem. Int. Ed. 2011, 50, 4219-4221.

136. Rees, N. V.; Zhou, Y. G.; Compton, R. G., The Aggregation of Silver Nanoparticles in Aqueous Solution Investigated via Anodic Particle Coulometry. ChemPhysChem 2011, 12, 1645-1647.

137. Anderson, T. J.; Zhang, B., Single-Nanoparticle Electrochemistry through Immobilization and Collision. Acc. Chem. Res. 2016, 49, 2625-2631.

138. German, S. R.; Hurd, T. S.; White, H. S.; Mega, T. L., Sizing Individual Au Nanoparticles in Solution with Sub-Nanometer Resolution. ACS Nano 2015, 9, 71867194.

139. Xiao, X.; Bard, A. J., Observing Single Nanoparticle Collisions at an Ultramicroelectrode by Electrocatalytic Amplification. J. Am. Chem. Soc. 2007, 129, 96109612.

140. Ngamchuea, K.; Clark, R. O.; Sokolov, S. V.; Young, N. P.; Batchelor-McAuley, C.; Compton, R. G., Single Oxidative Collision Events of Silver Nanoparticles: Understanding the Rate-Determining Chemistry. Chem. Eur. J. 2017, 23, 16085-16096.

141. Fu, K.; Han, D.; Crouch, G. M.; Kwon, S. R.; Bohn, P. W., Voltage-Gated Nanoparticle Transport and Collisions in Attoliter-Volume Nanopore Electrode Arrays. Small 2018, 1703248, 1-10.

142. McKelvey, K.; Edwards, M. A.; White, H. S., Resistive Pulse Delivery of Single Nanoparticles to Electrochemical Interfaces. J. Phys. Chem. Lett. 2016, 7, 3920-3924.

143. Lan, W.-J.; Kubeil, C.; Xiong, J.-W.; Bund, A.; White, H. S., Effect of Surface Charge on the Resistive Pulse Waveshape during Particle Translocation through Glass Nanopores. J. Phys. Chem. C 2014, 118, 2726-2734.

144. Chaudhari, V. R.; Hassan, P. A.; Haram, S. K., Size-Dependent Quantized Double Layer Charging of Monolayer-Protected Silver Nanoparticles. New J. Chem. 2014, 38, 1761-1767.

145. Hicks, J. F.; Miles, D. T.; Murray, R. W., Quantized Double-Layer Charging of Highly Monodisperse Metal Nanoparticles. J. Am. Chem. Soc. 2002, 124, 13322-13328.

146. Masitas, R. A.; Zamborini, F. P., Oxidation of Highly Unstable <4 Nm Diameter Gold Nanoparticles $850 \mathrm{mV}$ Negative of the Bulk Oxidation Potential. J. Am. Chem. Soc. 2012, 134, 5014-5017.

147. Henglein, A., Physicochemical Properties of Small Metal Particles in Solution:" Microelectrode" Reactions, Chemisorption, Composite Metal Particles, and the Atom-ToMetal Transition. J. Phys. Chem. 1993, 97, 5457-5471.

148. Plieth, W., Electrochemical Properties of Small Clusters of Metal Atoms and Their Role in the Surface Enhanced Raman Scattering. J. Phys. Chem. 1982, 86, 3166-3170. 
149. Tang, L.; Li, X.; Cammarata, R. C.; Friesen, C.; Sieradzki, K., Electrochemical Stability of Elemental Metal Nanoparticles. J. Am. Chem. Soc. 2010, 132, 11722-11726. 150. Han, D.; Kim, S.-S.; Kim, Y.-R.; Sohn, B.-H.; Chung, T. D., Surface coverage and size effects on electrochemical oxidation of uniform gold nanoparticles. Electrochem. Commun. 2015, 53, 11-14.

151. Ward Jones, S. E.; Campbell, F. W.; Baron, R.; Xiao, L.; Compton, R. G., Particle Size and Surface Coverage Effects in the Stripping Voltammetry of Silver Nanoparticles: Theory and Experiment. J. Phys. Chem. C 2008, 112, 17820-17827.

152. Brainina, K. Z.; Galperin, L. G.; Vikulova, E. V.; Stozhko, N. Y.; Murzakaev, A. M.; Timoshenkova, O. R.; Kotov, Y. A., Gold Nanoparticles Electrooxidation: Comparison of Theory and Experiment. J. Solid State Electrochem. 2011, 15, 1049-1056.

153. Brainina, K. Z.; Galperin, L. G.; Galperin, A. L., Mathematical Modeling and Numerical Simulation of Metal Nanoparticles Electrooxidation. J. Solid State Electrochem. 2010, 14, 981-988.

154. Toh, H. S.; Batchelor-McAuley, C.; Tschulik, K.; Uhlemann, M.; Crossley, A.; Compton, R. G., The Anodic Stripping Voltammetry of Nanoparticles: Electrochemical Evidence for the Surface Agglomeration of Silver Nanoparticles. Nanoscale 2013, 5, 488493.

155. Masitas, R. A.; Khachian, I. V.; Bill, B. L.; Zamborini, F. P., Effect of Surface Charge and Electrode Material on the Size-Dependent Oxidation of Surface-Attached Metal Nanoparticles. Langmuir 2014, 30, 13075-84.

156. Allen, S. L.; Sharma, J. N.; Zamborini, F. P., Aggregation-Dependent Oxidation of Metal Nanoparticles. J. Am. Chem. Soc. 2017, 139, 12895-12898.

157. Janz, A.; Kockritz, A.; Yao, L.; Martin, A., Fundamental Calculations on the Surface Area Determination of Supported Gold Nanoparticles by Alkanethiol Adsorption. Langmuir 2010, 26, 6783-6789.

158. Gadogbe, M.; Ansar, S. M.; He, G.; Collier, W. E.; Rodriguez, J.; Liu, D.; Chu, I. W.; Zhang, D., Determination of Colloidal Gold Nanoparticle Surface Areas, Concentrations, and Sizes through Quantitative Ligand Adsorption. Anal. Bioanal. Chem. 2013, 405, 413-22.

159. Wang, T. C.; Bury, W.; Gómez-Gualdrón, D. A.; Vermeulen, N. A.; Mondloch, J. E.; Deria, P.; Zhang, K.; Moghadam, P. Z.; Sarjeant, A. A.; Snurr, R. Q., Ultrahigh Surface Area Zirconium Mofs and Insights into the Applicability of the BET Theory. J. Am. Chem. Soc. 2015, 137, 3585-3591.

160. Walton, K. S.; Snurr, R. Q., Applicability of the BET Method for Determining Surface Areas of Microporous Metal-Organic Frameworks. J. Am. Chem. Soc. 2007, 129, 8552-8556.

161. Green, C. L.; Kucernak, A., Determination of the Platinum and Ruthenium Surface Areas in Platinum- Ruthenium Alloy Electrocatalysts by Underpotential Deposition of Copper. I. Unsupported Catalysts. J. Phys. Chem. B 2002, 106, 1036-1047.

162. Ogura, K.; Haruyama, S.; Nagasaki, K., The Electrochemical Oxidation and Reduction of Gold. J. Electrochem. Soc. 1971, 118, 531-535.

163. Elliott III, E. W.; Glover, R. D.; Hutchison, J. E., Removal of Thiol Ligands from Surface-Confined Nanoparticles Without Particle Growth or Desorption. ACS Nano 2015, 9, 3050-3059.

164. Park, J.-W.; Shumaker-Parry, J. S., Structural Study of Citrate Layers on Gold Nanoparticles: Role of Intermolecular Interactions in Stabilizing Nanoparticles. J. Am. Chem. Soc. 2014, 136, 1907-1921. 
165. Tan, J.; Liu, R.; Wang, W.; Liu, W.; Tian, Y.; Wu, M.; Huang, Y., Controllable Aggregation and Reversible $\mathrm{pH}$ Sensitivity of AuNPs Regulated by Carboxymethyl Cellulose. Langmuir 2010, 26, 2093-2098.

166. Hormozi-Nezhad, M. R.; Abbasi-Moayed, S., A Visual Colorimetric Probe for Naked-Eye Detection of Pamidronate Disodium in Human Plasma Based on Aggregation of Citrate-Capped Gold Nanoparticles. Plasmonics 2015, 10, 971-978.

167. Ross, M. B.; Ashley, M. J.; Schmucker, A. L.; Singamaneni, S.; Naik, R. R.; Schatz, G. C.; Mirkin, C. A., Structure-Function Relationships for Surface-Enhanced Raman Spectroscopy-Active Plasmonic Paper. J. Phys. Chem. C 2016, 120, 20789-20797.

168. Barberio, M.; Antici, P., In Situ Study of Nucleation and Aggregation Phases for Nanoparticles Grown by Laser-Driven Methods. Sci. Rep. 2017, 7, 41372.

169. Manthiram, K.; Surendranath, Y.; Alivisatos, A. P., Dendritic Assembly of Gold Nanoparticles during Fuel-Forming Electrocatalysis. J. Am. Chem. Soc. 2014, 136, 72377240.

170. Ghimire, G.; Coceancigh, H.; Yi, Y.; Ito, T., Electrochemical Characterization and Catalytic Application of Gold-Supported Ferrocene-Containing Diblock Copolymer Thin Films in Ethanol Solution. ACS Appl. Mater. Interfaces 2017, 9, 2906-2913.

171. Feng, J.-J.; Liu, L.; Huang, H.; Wang, A.-J., Poly(lonic Liquid)-Assisted One-Pot Synthesis of Au Hyperbranched Architectures for Enhanced SERS Performances. Sens. Actuator B-Chem. 2017, 238, 91-97.

172. Mei, L.-P.; Feng, J.-J.; Huang, S.-S.; Shi, Y.-C.; Wang, A.-J., Highly Sensitive Electrochemical Determination of Azathioprine using A Glassy Carbon Electrode Modified with Au Neuronal-Like Nanostructures. Sens. Actuator B-Chem. 2017, 240, 996-1002.

173. Chuang, M.-K.; Lin, S.-W.; Chen, F.-C.; Chu, C.-W.; Hsu, C.-S., Gold NanoparticleDecorated Graphene Oxides for Plasmonic-Enhanced Polymer Photovoltaic Devices. Nanoscale 2014, 6, 1573-1579.

174. Mistry, H.; Reske, R.; Zeng, Z.; Zhao, Z.-J.; Greeley, J.; Strasser, P.; Cuenya, B. R., Exceptional Size-Dependent Activity Enhancement in the Electroreduction of $\mathrm{CO}_{2} \mathrm{Over}$ Au Nanoparticles. J. Am. Chem. Soc. 2014, 136, 16473-16476.

175. Brainina, K. Z.; Galperin, L. G.; Vikulova, E. V.; Stozhko, N. Y.; Murzakaev, A. M.; Timoshenkova, O. R.; Kotov, Y. A., Gold Nanoparticles Electrooxidation: Comparison of Theory and Experiment. J. Solid State Electrochem. 2010, 15, 1049-1056.

176. Ivanova, O. S.; Zamborini, F. P., Electrochemical Size Discrimination of Gold Nanoparticles Attached to Glass/Indium-Tin-Oxide Electrodes by Oxidation in BromideContaining Electrolyte. Analytical Chemistry 2010, 82, 5844-5850.

177. Masitas, R. A.; Zamborini, F. P., Oxidation of Highly Unstable $<4 \mathrm{~nm}$ Diameter Gold Nanoparticles $850 \mathrm{mV}$ Negative of the Bulk Oxidation Potential. J. Am. Chem. Soc. 2012, 134, 5014-5017.

178. Pattadar, D. K.; Zamborini, F. P., Size Stability Study of Catalytically Active Sub-2 nm Diameter Gold Nanoparticles Synthesized with Weak Stabilizers. J. Am. Chem. Soc. 2018, 140, 14126-14133.

179. Redmond, P. L.; Hallock, A. J.; Brus, L. E., Electrochemical Ostwald Ripening of Colloidal Ag Particles on Conductive Substrates. Nano Lett. 2005, 5, 131-135.

180. Brainina, K. Z.; Galperin, L. G.; Kiryuhina, T. Y.; Galperin, A. L.; Stozhko, N. Y.; Murzakaev, A. M.; Timoshenkova, O. R., Silver Nanoparticles Electrooxidation: Theory and Experiment. J. Solid State Electrochem. 2012, 16, 2365-2372.

181. Pattadar, D. K.; Sharma, J. N.; Mainali, B. P.; Zamborini, F. P., Anodic Stripping Electrochemical Analysis of Metal Nanoparticles. Curr. Opin. Electrochem. 2019, 13, 147156. 
182. Pattadar, D. K.; Zamborini, F. P., Halide-Dependent Dealloying of Cux/Auy Core/Shell Nanoparticles for Composition Analysis by Anodic Stripping Voltammetry. J. Phys. Chem. C 2019.

183. Sieradzki, K., Curvature Effects in Alloy Dissolution. J. Electrochem. Soc. 1993, 140, 2868-2872.

184. Borm, P.; Klaessig, F. C.; Landry, T. D.; Moudgil, B.; Pauluhn, J.; Thomas, K.; Trottier, R.; Wood, S., Research Strategies for Safety Evaluation of Nanomaterials, Part V: Role of Dissolution in Biological Fate and Effects of Nanoscale Particles. Toxicol. Sci. 2006, 90, 23-32.

185. Kuo, C. L.; Hwang, K. C., Does Morphology of a Metal Nanoparticle Play a Role in Ostwald Ripening Processes? Chem. Mater. 2013, 25, 365-371.

186. Trindell, J. A.; Clausmeyer, J.; Crooks, R. M., Size Stability and $\mathrm{H}_{2} / \mathrm{CO}$ Selectivity for $\mathrm{Au}$ Nanoparticles During Electrocatalytic $\mathrm{CO}_{2}$ Reduction. J. Am. Chem. Soc. 2017, 139, 16161-16167.

187. Masitas, R. A.; Allen, S. L.; Zamborini, F. P., Size-Dependent Electrophoretic Deposition of Catalytic Gold Nanoparticles. J. Am. Chem. Soc. 2016, 138, 15295-15298.

188. Jiang, Y.; Zheng, B.; Du, J.; Liu, G.; Guo, Y.; Xiao, D., Electrophoresis Deposition Of Ag Nanoparticles On $\mathrm{TiO}_{2}$ Nanotube Arrays Electrode for Hydrogen Peroxide Sensing. Talanta 2013, 112, 129-135.

189. Zhang, H.; Cadusch, J.; Kinnear, C.; James, T.; Roberts, A.; Mulvaney, P., Direct Assembly of Large Area Nanoparticle Arrays. ACS Nano 2018, 12, 7529-7537.

190. Sibakoti, T. R.; Stinger, C. R.; Adhihetty, P. K.; Zamborini, F. P.; Nantz, M. H., Tunable Aminooxy-Functionalized Monolayer-Protected Gold Clusters for Nonpolar and Aqueous Oximation Reactions. Part. Part. Syst. Charact. 0, 1900093.

191. Allen, S. L.; Zamborini, F. P., Size-Selective Electrophoretic Deposition of Gold Nanoparticles Mediated by Hydroquinone Oxidation. Langmuir 2019, 35, 2137-2145.

192. Antolini, E., Carbon Supports for Low-Temperature Fuel Cell Catalysts. Appl. Catal. B. Environ. 2009, 88, 1-24.

193. Sevim Yılmaz, M.; Kaplan, B. Y.; Metin, Ö.; Gürsel, S. A., A Facile Synthesis and Assembly of Ultrasmall Pt Nanoparticles on Reduced Graphene Oxide-Carbon Black Hybrid for Enhanced Performance in PEMFC. Mater. Des. 2018, 151, $29-36$.

194. Luo, J.; Maye, M. M.; Petkov, V.; Kariuki, N. N.; Wang, L.; Njoki, P.; Mott, D.; Lin, Y.; Zhong, C.-J., Phase Properties of Carbon-Supported Gold-Platinum Nanoparticles with Different Bimetallic Compositions. Chem. Mater. 2005, 17, 3086-3091.

195. Azeredo, B. P.; Yeratapally, S. R.; Kacher, J.; Ferreira, P. M.; Sangid, M. D., An Experimental and Computational Study of Size-Dependent Contact-Angle Of Dewetted Metal Nanodroplets Below Its Melting Temperature. Appl. Phys. Lett. 2016, 109, 213101. 196. Ostojic, N.; Duan, Z.; Galyamova, A.; Henkelman, G.; Crooks, R. M., Electrocatalytic Study of the Oxygen Reduction Reaction at Gold Nanoparticles in the Absence and Presence of Interactions with SnOx Supports. J. Am. Chem. Soc. 2018, 140, $13775-13785$.

197. Zhan, W.-W.; Zhu, Q.-L.; Xu, Q., Dehydrogenation of Ammonia Borane by Metal Nanoparticle Catalysts. ACS Catalysis 2016, 6, 6892-6905.

198. Liu, J.; Dai, M.; Wang, T.; Sun, P.; Liang, X.; Lu, G.; Shimanoe, K.; Yamazoe, N., Enhanced Gas Sensing Properties of SnO2 Hollow Spheres Decorated with $\mathrm{CeO} 2$ Nanoparticles Heterostructure Composite Materials. ACS Appl. Mater. Interfaces 2016, 8, 6669-6677.

199. Lan, G.; Ni, K.; Xu, Z.; Veroneau, S. S.; Song, Y.; Lin, W., Nanoscale MetalOrganic Framework Overcomes Hypoxia for Photodynamic Therapy Primed Cancer Immunotherapy. J. Am. Chem. Soc. 2018, 140, 5670-5673. 
200. Hejral, U.; Franz, D.; Volkov, S.; Francoual, S.; Strempfer, J.; Stierle, A., Identification of a Catalytically Highly Active Surface Phase for CO Oxidation over PtRh Nanoparticles under Operando Reaction Conditions. Phys. Rev. Lett. 2018, 120, 126101. 201. Chen, Q.; Zhang, X.; Su, S.; Xu, Z.; Li, N.; Li, Y.; Zhou, H.; Bao, M.; Yamamoto, Y.; Jin, T., Nanoporous Gold-Catalyzed Diboration of Methylenecyclopropanes via a Distal Bond Cleavage. ACS Catalysis 2018, 8, 5901-5906.

202. Liu, H.; Yang, Q., Feasible Synthesis of Etched Gold Nanoplates with Catalytic Activity and SERS Properties. CrystEngComm 2011, 13, 5488-5494.

203. Ab Kadir, R.; Rani, R. A.; Alsaif, M. M. Y. A.; Ou, J. Z.; Wlodarski, W.; O’Mullane, A. P.; Kalantar-zadeh, K., Optical Gas Sensing Properties of Nanoporous $\mathrm{Nb}_{2} \mathrm{O}_{5}$ Films. ACS Appl. Mater. Interfaces 2015, 7, 4751-4758.

204. Shi, Y.; Guo, B.; Corr, S. A.; Shi, Q.; Hu, Y.-S.; Heier, K. R.; Chen, L.; Seshadri, R.; Stucky, G. D., Ordered Mesoporous Metallic $\mathrm{MoO}_{2}$ Materials with Highly Reversible Lithium Storage Capacity. Nano Lett. 2009, 9, 4215-4220.

205. Jia, F.; Yu, C.; Ai, Z.; Zhang, L., Fabrication of Nanoporous Gold Film Electrodes with Ultrahigh Surface Area and Electrochemical Activity. Chem. Mater. 2007, 19, 36483653.

206. Chen, L.; Ji, F.; Xu, Y.; He, L.; Mi, Y.; Bao, F.; Sun, B.; Zhang, X.; Zhang, Q., HighYield Seedless Synthesis of Triangular Gold Nanoplates through Oxidative Etching. Nano Lett. 2014, 14, 7201-7206.

207. Xu, J.; Li, S.; Weng, J.; Wang, X.; Zhou, Z.; Yang, K.; Liu, M.; Chen, X.; Cui, Q.; Cao, M.; Zhang, Q., Hydrothermal Syntheses of Gold Nanocrystals: From Icosahedral to Its Truncated Form. Adv. Funct. Mater. 2008, 18, $277-284$.

208. Qi, Z.; Weissmüller, J., Hierarchical Nested-Network Nanostructure by Dealloying. ACS Nano 2013, 7, 5948-5954.

209. Chao, B. K.; Xu, Y.; Ho, H. C.; Yiu, P.; Lai, Y. C.; Shek, C. H.; Hsueh, C. H., GoldRich Ligament Nanostructure by Dealloying Au-Based Metallic Glass Ribbon for SurfaceEnhanced Raman Scattering. Sci Rep 2017, 7, 7485.

210. Zhong, S.-L.; Zhuang, J.; Yang, D.-P.; Tang, D., Eggshell Membrane-Templated Synthesis of 3D Hierarchical Porous Au Networks for Electrochemical Nonenzymatic Glucose Sensor. Biosens Bioelectron 2017, 96, 26-32.

211. Vega, A. A.; Newman, R. C., Nanoporous Metals Fabricated Through Electrochemical Dealloying of Ag-Au-Pt With Systematic Variation of Au: Pt Ratio. J. Electrochem. Soc. 2014, 161, C1-C10.

212. Rouya, E.; Cattarin, S.; Reed, M.; Kelly, R.; Zangari, G., Electrochemical Characterization of the Surface Area of Nanoporous Gold Films. J. Electrochem. Soc. 2012, 159, K97-K102.

213. Sharma, J.; Pattadar, D.; Mainali, B.; Zamborini, F. In Electrochemical Determination of Surface Area-To-Volume Ratio for Metal Nanoparticle Size Analysis, Abstracts of Papers, 255th ACS National Meeting Exposition, New Orleans, LA, United States, March 18-22, 2018.

214. Link, S.; Wang, Z. L.; El-Sayed, M. A., Alloy Formation of Gold-Silver Nanoparticles and the Dependence of the Plasmon Absorption on Their Composition. J. Phys. Chem. B 1999, 103, 3529-3533.

215. Tran, M.; DePenning, R.; Turner, M.; Padalkar, S., Effect of Citrate Ratio and Temperature on Gold Nanoparticle Size and Morphology. Mater. Res. Express 2016, 3, 105027.

216. Artymowicz, D. M.; Erlebacher, J.; Newman, R. C., Relationship between the Parting Limit for De-Alloying and A Particular Geometric High-Density Site Percolation Threshold. Philos. Mag. 2009, 89, 1663-1693. 


\title{
Appendix
}

\author{
Title: $\quad$ Size Determination of Metal NPs Based \\ on Electrochemically Measured \\ Surface-Area-to-Volume Ratios \\ Author: Jay N. Sharma, Dhruba K. Pattadar, \\ Badri P. Mainali, et al \\ Publication: Analytical Chemistry \\ Publisher: American Chemical Society \\ Date: $\quad$ Aug 1, 2018 \\ Copyright @ 2018, American Chemical Society
}

\section{PERMISSION/LICENSE IS GRANTED FOR YOUR ORDER AT NO CHARGE}

This type of permission/license, instead of the standard Terms \& Conditions, is sent to you because no fee is being charged for your order. Please note the following:

- Permission is granted for your request in both print and electronic formats, and translations.

- If figures and/or tables were requested, they may be adapted or used in part.

- Please print this page for your records and send a copy of it to your publisher/graduate school.

- Appropriate credit for the requested material should be given as follows: "Reprinted (adapted) with permission from (COMPLETE REFERENCE CITATION). Copyright (YEAR) American Chemical Society." Insert appropriate information in place of the capitalized words.

- One-time permission is granted only for the use specified in your request. No additional uses are granted (such as derivative works or other editions). For any other uses, please submit a new request. 


\author{
Title: Aggregation-Dependent \\ Oxidation of Metal \\ Nanoparticles \\ Author: Stacy L. Allen, Jay N. Sharma, \\ Francis P. Zamborini \\ Publication: Journal of the American \\ Chemical Society \\ Publisher: American Chemical Society \\ Date: Sep 1, 2017 \\ Copyright (c) 2017, American Chemical Society
}

Title: Aggregation-Dependent Oxidation of Metal Nanoparticles

Author: Stacy L. Allen, Jay N. Sharma, Francis $\mathrm{P}$. Zamborini

Publication: Journal of the American Chemical Society

Publisher: American Chemical Society

Date: Sep 1, 2017

Copyright (C) 2017, American Chemical Society

\title{
PERMISSION/LICENSE IS GRANTED FOR YOUR ORDER AT NO CHARGE
}

This type of permission/license, instead of the standard Terms \& Conditions, is sent to you because no fee is being charged for your order. Please note the following:

- Permission is granted for your request in both print and electronic formats, and translations.

- If figures and/or tables were requested, they may be adapted or used in part.

- Please print this page for your records and send a copy of it to your publisher/graduate school.

- Appropriate credit for the requested material should be given as follows: "Reprinted (adapted) with permission from (COMPLETE REFERENCE CITATION). Copyright (YEAR) American Chemical Society." Insert appropriate information in place of the capitalized words.

- One-time permission is granted only for the use specified in your request. No additional uses are granted (such as derivative works or other editions). For any other uses, please submit a new request. 


\author{
CURRICULUM VITTAE
}

\title{
Jay Narayan Sharma
}

2320 South Brook Street, Louisville, Kentucky 40292, USA

jay.sharma43@gmail.com

\section{Experience}

Graduate Teaching Assistant: Department of Chemistry, University of Louisville, Louisville, Kentucky, USA, August 2013-May 2019.

- Trained undergraduate students in general laboratory safety and techniques and monitored them while conducting their lab experiments.

- Presented prelab lectures before at the beginning of laboratory classes.

- Assisted with laboratory set-up before class and clean-up after class.

- Graded lab reports and proctored and graded class exams.

Graduate Research Assistant: Department of Chemistry, University of Louisville,

Louisville, Kentucky, USA, May 2019-July 2019.

- Developed a method for determination of the sizes of metal Au nanospheres based on electrochemically-measured SA-to-volume ratio.

- Studied the effect of nanoparticle assembly method and electrode material on the metal oxidation behavior and SA-to-volume ratio.

- Co-worked on a project studying the aggregation-dependent oxidation of metal NPs.

- Electrochemically-measured the SA-to-volume ratio of porous Au NPs formed by dealloying and correlated their porosity to their oxidation potential.

\section{Education}




\section{Summer 2019}

Ph.D. in Chemistry, Department of Chemistry, University of Louisville, Louisville, Kentucky, USA.

Thesis title: Electrochemical Determination of SA-to-Volume Ratios for Metal Nanoparticle Analysis

\section{Fall 2012}

Master's Degree in Pharmacy (MS-Pharmacy), Department of Pharmaceutics, Kamla Nehru College of Pharmacy, RTM Nagpur University, Nagpur, MH, India.

Thesis title: Formulation, development and evaluation of oral mucoadhesive delivery system

\section{Fall 2009}

Bachelor's Degree in Pharmacy (BS-Pharmacy), SKB College of Pharmacy, RTM Nagpur University, Nagpur, MH, India.

\section{Skills}

- Experienced in experiment design, writing and editing of scientific documents and communications for peer-reviewed research journals, conferences, symposia, departmental seminars and journal clubs.

- Strong expertise in electrochemical techniques (such as cyclic voltammetry (CV), linear sweep voltammetry (LSV), and chronoamperometry (CA)), Scanning Electron Microscopy (SEM), UV-vis spectroscopy, Fourier Transform Infrared (FTIR) spectroscopy, Gas Chromatography (GC), Atomic Absorption Spectroscopy (AAS), Atomic Force Microscopy (AFM), and Raman Spectroscopy, Differential Scanning Calorimetry (DSC) and Thermogravimetric Analysis (TGA)

- Ability to work independently as well as collaboratively in scientific teams with diverse backgrounds and research interests.

- Strong verbal and written communication skills.

\section{Achievements}


- Awarded Doctoral Dissertation Award (2018) by Graduate School of Education, University of Louisville, Louisville, KY

- Awarded Graduate Travel Fund (2018 and 2019) by Graduate Network in Arts and Sciences (GNAS), University of Louisville, Louisville, KY

- Junior Research Fellowship (2010-2012) All India Council for Technical Education (AICTE), Government of India for pursuing Master's dissertation research.

\section{Publications}

1. Sharma, J. N.; Pattadar, D. K.; Mainali, B. P.; Zamborini, F. P. Size Determination of Metal NPs Based on Electrochemically Measured SurfaceArea-to-Volume Ratios. Anal. Chem. 2018, 90, 9308-9314. https://pubs.acs.org/doi/10.1021/acs.analchem.8b01905

2. Pattadar, D. K.; ${ }^{1}$ Sharma, J. N.; Mainali, B. P.; Zamborini, F. P. The Impact of Assembly Method on the Surface area-to-Volume Ratio and Electrochemical Oxidation Potential of Metal NPs. J. Phys. Chem. C, 2019, DOI:

10.1021/acs.jpcc.9b06555 https://pubs.acs.org/doi/pdf/10.1021/acs.jpcc.9b06555

3. Pattadar, D.K.; Sharma, J.N.; Mainali, B.P.; Zamborini, F.P. Structural, Physical, and Chemical Characterization of Metal NPs by Anodic Stripping Electrochemical Analysis. Curr. Opin. Electrochem. 2019, 13, 147-156. https://www.sciencedirect.com/science/article/pii/S2451910318302047

4. Pattadar, D. K.; Mainali, B.P.; Sharma, J. N.; Zamborini, F. P. Understanding the Thermal Sintering Behavior of Sub $2 \mathrm{~nm}$ Diameter Gold NPs Synthesized with Weak Stabilizers. (Manuscript prepared)

5. Allen, S. L.; Sharma, J. N.; Zamborini, F. P. Aggregation-Dependent Oxidation of Metal NPs. J. Am. Chem. Soc. 2017, 139, 12895-12898.

https://pubs.acs.org/doi/abs/10.1021/jacs.7b05957?journalCode=jacsat

6. Sharma, J. N.; Zamborini, F. P. The Surface area-to-Volume Ratio and Oxidation Potential of AuAg Nanospheres After Ag Dealloying. (Manuscript prepared)

\section{Professional Trainings}

- "Intern", at Amcor Flexibles, Shelbyville, KY, USA (Summer 2017). 
- "Project intern", at Cadila Pharmaceuticals Pvt. Ltd, Dholka, Ahmedabad, Gujarat, India (2011).

\section{Languages Known}

English, Hindi, Marathi, Marwari 